University of Louisville

ThinkIR: The University of Louisville's Institutional Repository

Electronic Theses and Dissertations

$5-2013$

\title{
COUP-TFII and its interacting proteins in breast cancer and endocrine resistance.
}

Lacey Morgan Litchfield

University of Louisville

Follow this and additional works at: https://ir.library.louisville.edu/etd

\section{Recommended Citation}

Litchfield, Lacey Morgan, "COUP-TFII and its interacting proteins in breast cancer and endocrine resistance." (2013). Electronic Theses and Dissertations. Paper 839.

https://doi.org/10.18297/etd/839

This Doctoral Dissertation is brought to you for free and open access by ThinkIR: The University of Louisville's Institutional Repository. It has been accepted for inclusion in Electronic Theses and Dissertations by an authorized administrator of ThinkIR: The University of Louisville's Institutional Repository. This title appears here courtesy of the author, who has retained all other copyrights. For more information, please contact thinkir@louisville.edu. 


\title{
COUP-TFII AND ITS INTERACTING PROTEINS IN BREAST CANCER AND ENDOCRINE RESISTANCE
}

\author{
By \\ Lacey Morgan Litchfield \\ B.S., University of Louisville, 2008 \\ M.S., University of Louisville, 2011

\begin{abstract}
A Dissertation
Submitted to the Faculty of the

University of Louisville School of Medicine

in Partial Fulfillment of the Requirements

for the Degree of
\end{abstract}

Doctor of Philosophy

Department of Biochemistry and Molecular Biology

University of Louisville, School of Medicine

Louisville, KY

May 2013 
Copyright 2013 by Lacey M. Litchfield

All rights reserved 


\section{COUP-TFII AND ITS INTERACTING PROTEINS IN BREAST CANCER AND}

ENDOCRINE RESISTANCE

By

Lacey Morgan Litchfield

B.S., University of Louisville, 2008

M.S., University of Louisville, 2011

A Dissertation Approved on

April 5, 2013

by the following Thesis Committee:

Dr. Carolyn M. KGnge (Mentor)

Dr. Paula J. Bates

Dr. Barbara J. Clark

Dr. Chuan Hu

Dr. David W. Powell 


\section{ACKNOWLEDGEMENTS}

I would like to thank my mentor, Dr. Carolyn M. Klinge, for her invaluable guidance and support in my scientific growth. I would also like to thank the rest of my dissertation committee, Dr. Paula J. Bates, Dr. Barbara J. Clark, Dr. Chuan Hu, and Dr. David W. Powell, for their comments and advice regarding my project. I am sincerely grateful for the members of the Klinge laboratory in their support and assistance over the years. 


\section{ABSTRACT \\ COUP-TFII AND ITS INTERACTING PROTEINS IN BREAST CANCER AND ENDOCRINE RESISTANCE}

Lacey M. Litchfield

April 5, 2013

Chicken ovalbumin upstream promoter transcription factor II (COUP-TFII) is an orphan nuclear receptor that functions as either a transcriptional activator or repressor. COUP-TFII expression is reduced in endocrine-resistant breast cancer cells and overexpression of COUP-TFII restores the ability of antiestrogens to inhibit cell proliferation. In this study, I tested the hypothesis that the activity of COUP-TFII in maintenance of endocrine sensitivity and cell differentiation is dependent on its interacting proteins. A direct interaction was identified between COUP-TFII and the phosphoprotein nucleolin, which was found to function as a coregulator for COUP-TFIImediated transcription. COUP-TFII increased the expression of the tumor suppressor retinoic acid receptor B2 (RARB2) in a nucleolin-dependent manner. COUP-TFII and nucleolin expression were correlated in estrogen receptor $\alpha(\mathrm{ER} \alpha)+$ invasive ductal carcinomas. COUP-TFII expression was inversely correlated with patient tumor grade.

Aberrant activation of the NFKB pathway has also been implicated in endocrine resistance. COUP-TFII transfection suppressed NFKB activity in endocrine-resistant breast cancer cells. COUP-TFII overexpression also reduced the expression of NFKB 
target genes (IL6, ICAM1, TNFAIP3, and CCL2) and subunits (NFKB1, REL, RELA, and RELB) through a mechanism involving interaction of COUP-TFII with NFKB subunits RelB and NFאB1 to inhibit NFאB DNA-binding. COUP-TFII also reduced the ability of coactivators SRC-1, SRC-3, and CBP to enhance NFKB activity. An inverse correlation between COUP-TFII and IL6, ICAM1, TNFAIP3, NFKB2, REL, RELA, and RELB was observed in breast tumors from tamoxifen-treated breast cancer patients. The combination of COUP-TFII overexpression, NFkB inhibition, and tamoxifen treatment inhibited the growth of endocrine-resistant breast cancer cells. Endocrine-resistant breast cancer cells were more sensitive to treatment with an $\mathrm{NF \kappa B}$ inhibitor than endocrinesensitive cells, reflecting their reliance on the NFKB pathway for survival.

To further explore the role of COUP-TFII-interacting proteins in endocrine sensitivity, mass spectrometry was used to identify proteins that interacted with COUPTFII specifically when breast cancer cells were treated with tamoxifen versus vehicle control. HSP27 was selected for follow-up from the proteins identified as interacting specifically with COUP-TFII in cells treated with tamoxifen. HSP27 expression was reduced in an endocrine-resistant breast cancer cell line that has undergone epithelial to mesenchymal transition, LY2, as well as in a trastuzumab-resistant breast cancer cell line, JIMT-1. A concomitant reduction in COUP-TFII expression was also observed in these cells. Future studies will explore the role of COUP-TFII-HSP27 interaction in resistance to tamoxifen and trastuzumab. COUP-TFII may be both a useful biomarker to predict tamoxifen and/or trastuzumab sensitivity as well as a target to restore sensitivity to resistant cells. 


\section{TABLE OF CONTENTS}

ACKNOWLEDGEMENTS ......................................................................................................... iii

ABSTRACT ................................................................................................................................... iv

LIST OF TABLES ........................................................................................................................... ix

LIST OF FIGURES ................................................................................................................................

CHAPTER I - BREAST CANCER AND ENDOCRINE RESISTANCE .....................1

BREAST STRUCTURE AND DEVELOPMENT …………………………….........

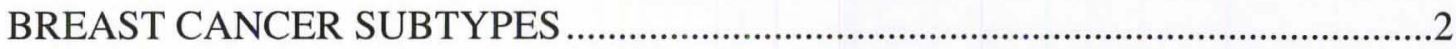

ESTROGEN RECEPTOR FUNCTION ...........................................................

BREAST CANCER TREATMENT …………………….................................6

MECHANISMS OF ENDOCRINE RESISTANCE................................................

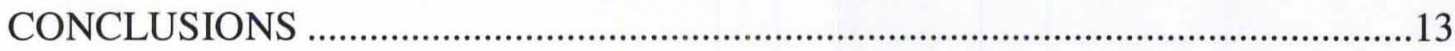

CHAPTER II - MULTIPLE ROLES OF COUP-TFII IN CANCER INITIATION

AND PROGRESSION.........................................................................................................14

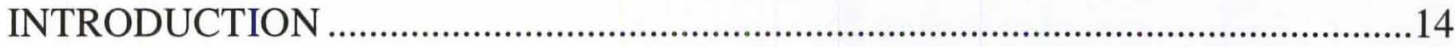

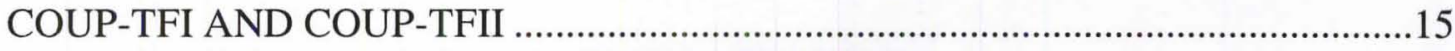

COUP-TFII REGULATION OF GENE EXPRESSION ……………………….......17

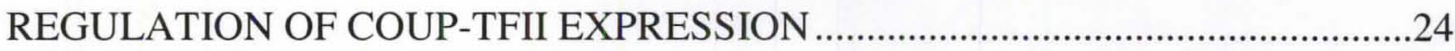

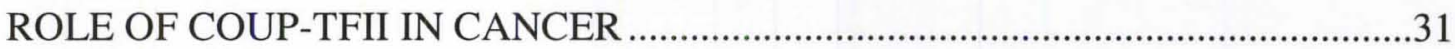

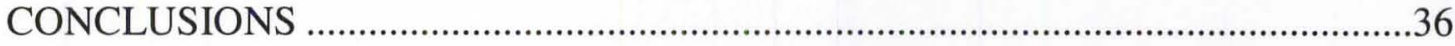

DISSERTATION SPECIFIC AIMS AND HYPOTHESES ..............................................37

CHAPTER III - IDENTIFICATION AND CHARACTERIZATION OF

NUCLEOLIN AS A COUP-TFII COACTIVATOR OF RETINOIC ACID

RECEPTOR $\beta$ TRANSCRIPTION IN BREAST CANCER CELLS............................40

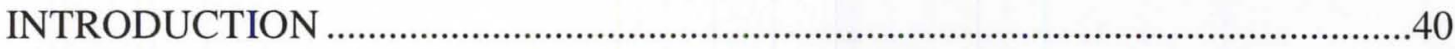

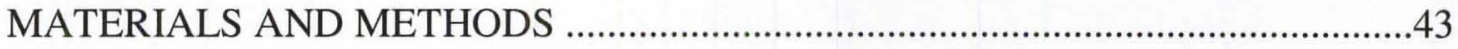

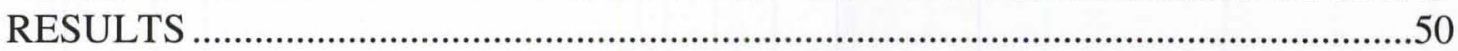

Identification of COUP-TFII-associated proteins .....................................................50

Endogenous COUP-TFII and nucleolin interact in MCF-7 and T47D cells ............54

Direct interaction of COUP-TFII with the RGG domain of nucleolin .......................56

Immunohistochemical COUP-TFII and nucleolin staining in human breast tissue and tumor tissue microarrays ...............................................................................56

atRA enhances COUP-TFII binding to the RARB2 promoter ....................................60 
AS1411 inhibits COUP-TFII-stimulated RARB2 gene expression in MCF-7 and T47D breast cancer cells......

RARB2 expression is inhibited by nucleolin or COUP-TFII knockdown ...................65

Effects of AS1411 on nucleolin-COUP-TFII nuclear interaction ..............................65

DISCUSSION

\section{CHAPTER IV - COUP-TFII INHIBITS NFKB ACTIVATION IN BREAST} CANCER CELLS AND RESTORES ENDOCRINE SENSITIVITY ...........................72

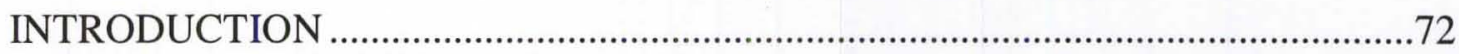

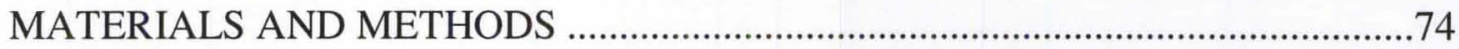

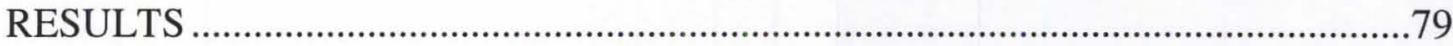

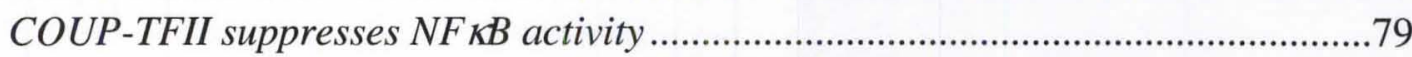

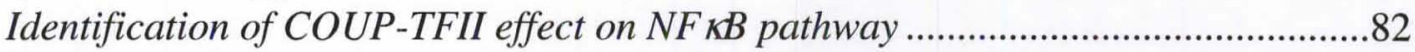

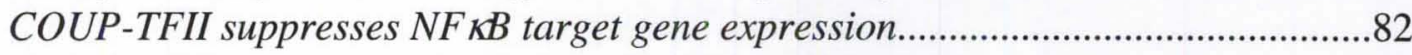

COUP-TFII suppresses $N F K B$ subunit gene expression ..........................................86

COUP-TFII and NF KB expression are inversely correlated in breast cancer patient

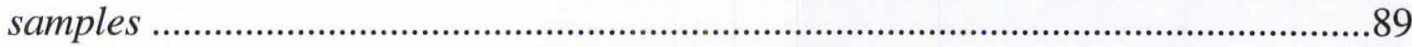

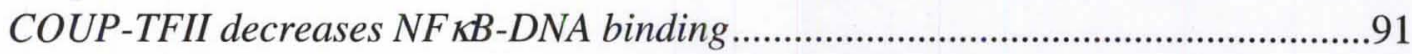

COUP-TFII inhibits RelB, RelA, and p50 NF KB activation.....................................94

COUP-TFII interacts with NF $K B$ subunits ...........................................................94

Inhibition of coactivator function by COUP-TFII reduces $N F K B$ transcriptional

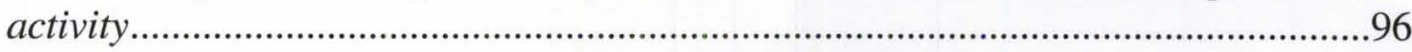

Endocrine-resistant cells are sensitive to NF kB-inhibition ....................................96

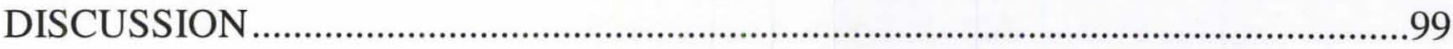

\section{CHAPTER V - IDENTIFICATION OF COUP-TFII-INTERACTING PROTEINS}

IN TAMOXIFEN-TREATED BREAST CANCER CELLS ..........................................102

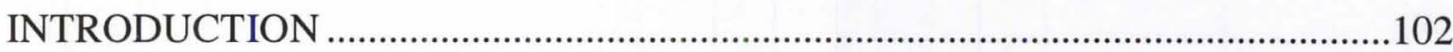

MATERIALS AND METHODS ………………………………………….......103

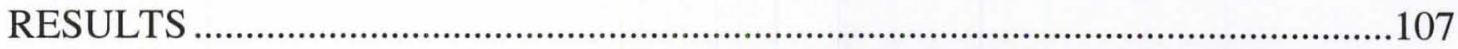

Identification of COUP-TFII-associated proteins in ethanol-and tamoxifen-treated cells ...........................................................................................................107

Functional Annotation Clustering of COUP-TFII-interacting proteins ..................112

Endogenous nuclear interaction of COUP-TFII with HSP27 ...............................115

Regulation of HSP27 by estradiol and tamoxifen in endocrine-resistant breast cancer cells ...................................................................................................118

HSP27 and COUP-TFII expression is reduced in endocrine-resistant and trastuzumab-resistant breast cancer cells ...............................................................119

COUP-TFII/HSP27 and HER2 expression are inversely correlated .......................120

DISCUSSION .......................................................................................................123

CHAPTER VI - RESEARCH IMPLICATIONS ..............................................................126

REFERENCES …..........................................................................................................................131

APPENDIX I - SUPPLEMENTARY MATERIAL_........................................................156 


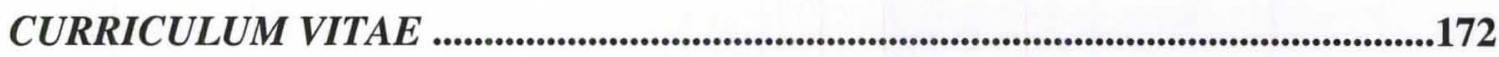




\section{LIST OF TABLES}

Table 1. List of COUP-TFII-regulated genes identified by Ingenuity Pathway Analysis.22

Table 2. Identification of COUP-TFII interacting proteins in MCF-7 cells.....................52

Table 3. COUP-TFII staining in breast tumor microarray. ............................................59

Table 4. Correlation of COUP-TFII expression to NFKB target and subunit gene expression in ER $\alpha+$ breast cancer patients treated with tamoxifen..................................90 Table 5. COUP-TFII-interacting proteins in ethanol- and tamoxifen-treated MCF-7 cells.

Table 6. Functional Annotation Clustering of COUP-TFII-interacting proteins. ...........113 


\section{LIST OF FIGURES}

Figure 1. Model of estrogen action in the breast and inhibition by aromatase inhibitors and antiestrogens. ................................................................................................

Figure 2. Comparison of COUP-TFI and COUP-TFII protein homology.........................16

Figure 3. COUP-TFII $(N R 2 F 2)$ target genes. ……………...............................................21

Figure 4. Regulation of COUP-TFII expression................................................................25

Figure 5. Insulin treatment does not affect COUP-TFII expression in human breast cancer

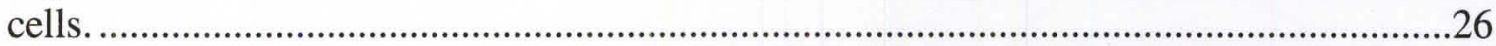

Figure 6. Endogenous nuclear nucleolin-COUP-TFII interaction in MCF-7 and T47D

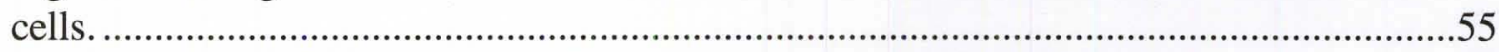

Figure 7. COUP-TFII and nucleolin in breast cancer tissue microarrays............................58

Figure 8. COUP-TFII increases RAR $\beta 2$ transcription in MCF-7 cells. ..............................62

Figure 9. Regulation of RAR $\beta 2$ transcription.................................................................64

Figure 10. Reduction of COUP-TFII or nucleolin decreases RAR $\beta 2$ transcription in

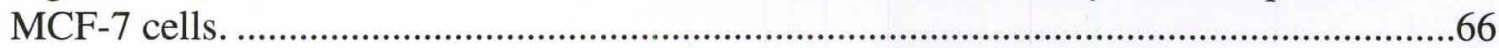

Figure 11. Effects of AS1411 on nuclear nucleolin-COUP-TFII interaction....................67

Figure 12. Model of nucleolin-COUP-TFII interaction and upregulation of RARB2 expression. .......................................................................................................

Figure 13. COUP-TFII suppresses NFKB activity in LCC9 cells. .....................................81

Figure 14. COUP-TFII inhibits expression of multiple genes in the NFKB pathway.......84

Figure 15. COUP-TFII inhibits expression of NFKB target genes....................................85

Figure 16. COUP-TFII inhibits mRNA expression of NFKB subunits in LCC9 cells......87

Figure 17. COUP-TFII inhibits protein expression of NFKB subunits...............................8

Figure 18. COUP-TFII decreases NFKB-DNA binding.....................................................92

Figure 19. COUP-TFII interacts with NFKB subunits and inhibits coactivator function in

$\mathrm{NF} \kappa \mathrm{B}$ activation.

Figure 20. Overexpression of COUP-TFII increases endocrine sensitivity in breast cancer

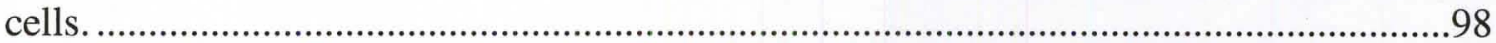

Figure 21. Efficiency of COUP-TFII-FLAG purification by FLAG affinity gel............109 Figure 22. Endogenous nuclear interaction of COUP-TFII with HSP27 and calmodulin in MCF-7 cells.

Figure 23. Regulation of HSP27 expression by $\mathrm{E}_{2}$ and 4-OHT in TAMS and TAMR cells.

Figure 24. Expression of HSP27, HER2, and COUP-TFII in endocrine-resistant and trastuzumab-resistant cells. 


\section{CHAPTER I - BREAST CANCER AND ENDOCRINE RESISTANCE}

\section{BREAST STRUCTURE AND DEVELOPMENT}

The complex branched structure of the adult mammary gland is composed of two basic cellular compartments: luminal and basal cells. Luminal epithelial cells line the lumen of the mammary duct, while the basal compartment is composed of progenitor cells and contractile myoepithelial cells that do not directly contact the ductal lumen. Coordinated paracrine signaling as well as structural connections between these two compartments are necessary for both the proper development and function of the mammary gland [1].

In the late 1950s, endocrine ablation experiments in rats and mice demonstrated that sequential timing of treatment with 17- $\beta$-estradiol $\left(\mathrm{E}_{2}\right)$, progesterone, and prolactin, in the presence of cortisol and growth hormone, were the minimal hormones necessary for mammary gland development [1]. Sequential hormone excretion and/or hormone receptor expression is required for the formation of proper glandular structure. Estrogens mediate the beginning of ductal branching and elongation as well as initial side branching, followed by progesterone regulation of continued side branching and alveoli formation. Alveologenesis is completed by prolactin, which also regulates milk production during the late stages of pregnancy [1]. Milk is produced in the lobules and transported through the ductal system. The ducts and ductal-lobular units are extended during puberty and further expand during pregnancy and lactation [2]. Aberrant 
proliferation can occur in both the duct and lobule structures to form ductal carcinoma or lobular carcinoma, respectively, although ductal carcinomas are more common, i.e., $75 \%$ of invasive breast cancers are ductal in origin, while $11 \%$ are lobular (the remaining $14 \%$ comprise a heterogeneous set of less common classifications) [3].

\section{BREAST CANCER SUBTYPES}

Breast cancer is the most commonly diagnosed cancer in women, and the leading cause of cancer death in females aged 20-59 years [4]. Breast cancers can be divided into 5 basic subtypes based on their distinct gene expression data: normal breast-like, luminal A, luminal B, ERBB2+, and basal [5-7]. The luminal A and luminal B groups express a gene signature similar to that of the luminal epithelial cells of normal mammary tissue, while the basal subtype is more reminiscent of basal epithelial cells [6,7]. Luminal B is distinguished from luminal A by higher expression of the cell proliferation marker Ki67, a biomarker associated with recurrence, relapse, and overall worse outcome in breast cancer patients [8-11]. The ERBB2+ group is defined by overexpression of the HER2/neu (ERBB2) receptor, a member of the epidermal growth factor receptor (EGFR) family that is ligand-independent and constitutively active, a known oncogenic transformation associated with poor outcome in breast cancer patients [12]. Normal breast-like cancers express a gene signature similar to that of adipose tissue, basal epithelial cells, and other nonepithelial components of the breast [5,7]. Breast cancer subtype is also indicative of clinical outcome, with basal and ERBB2+ subtypes associated with a statistically significant decrease in survival $[5,6]$. Luminal A tumors 
show the highest estrogen receptor $\alpha(E R \alpha)$ expression of the five subtypes, while $E R \alpha$ cancers generally fall in the ERBB2+ or basal subtypes [6,7].

\section{ESTROGEN RECEPTOR FUNCTION}

Estrogen's action as the ovarian hormone responsible for maintenance of estrus was first characterized in 1923 by Allen and Doisy using partially purified hormone from the hog ovary [13]. Further investigation revealed three forms of estrogen: estrone, estriol, and estradiol, with estradiol being the primary form in premenopausal women [14]. A receptor for estradiol (ER $\alpha)$ was first reported in 1962 [15], with discovery of a second subtype, ER $\beta$, following in 1996 [16]. Estradiol binding to ER $\alpha$ results in a conformational change that activates the receptor, leading to its dimerization. Upon formation of a dimer, ER $\alpha$ translocates to the nucleus wherein it binds to estrogen response elements (EREs) in target gene promoters (Figure 1) [17,18]. ER $\alpha$ can also bind to DNA through a tethering mechanism, wherein a transcription factor such as AP-1, e.g., $\mathrm{c}-\mathrm{Jun} / \mathrm{Fos}$, binds to specific target sequences and $\mathrm{ER} \alpha$ then binds through proteinprotein interaction with AP-1 $[19,20]$. Such a tethering mechanism can also occur between $\mathrm{ER} \alpha$ and $\mathrm{Sp} 1$ [21,22]. Binding of $\mathrm{ER} \alpha$ and associated coactivators, e.g., members of the SRC family or $\mathrm{CBP} / \mathrm{p} 300$, leads to recruitment of RNA polymerase II for transcription initiation [21,23]. Work by Carroll and Brown using chromatin immunoprecipitation (ChIP)-sequencing (ChIP-seq) technology has identified a large set of estrogen-responsive genes, and expanded understanding of ERE location to include sequences present in far upstream enhancer regions which exert their influence on target gene transcription through complex chromatin looping [24,25]. Many identified ER $\alpha$ - 
responsive genes have roles involved in cell proliferation and survival, cell cycle progression, growth factor signaling, and hormone synthesis [26]. ER $\beta$ functions in much the same way to bind to target gene DNA and promote gene transcription. ER $\alpha$ and $E R \beta$ share many EREs in common, though ER $\alpha$ takes preference in binding if both receptors are activated by ligand $[27,28]$. ER $\alpha$ and ER $\beta$ are both activated by estradiol binding; however, there have been receptor-selective endogenous ligands, e.g, $5 \alpha$ -

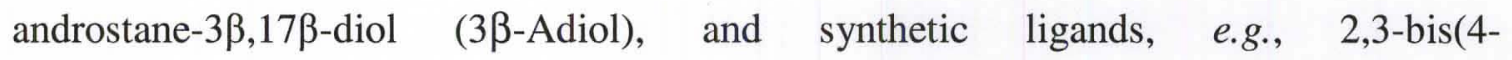
hydroxyphenyl)propionitrile (DPN) for ER $\beta$ identified [29,30]. ER $\alpha$ and ER $\beta$ may also recruit different coactivators, or corepressors instead of coactivators, resulting in alterations in their effects on downstream gene expression [29,31]. Rather than the proliferative activity of $E R \alpha, E R \beta$ has been reported to play a growth inhibitory role in many cell types $[29,32]$. 


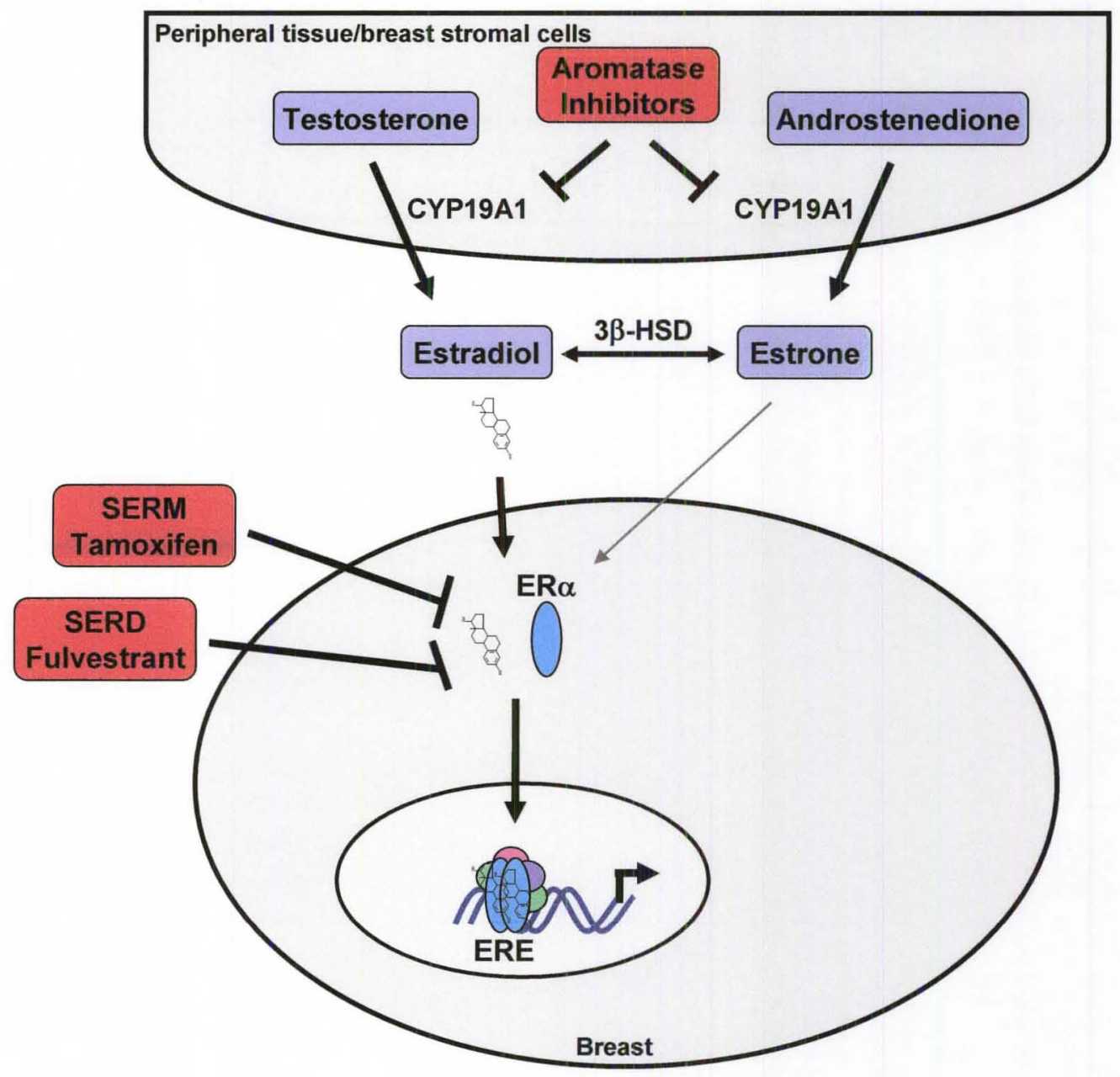

Figure 1. Model of estrogen action in the breast and inhibition by aromatase inhibitors and antiestrogens.

In breast cells, estradiol binds $\mathrm{ER} \alpha$ leading to its dimerization and subsequent DNA binding in the cell nucleus to regulate target gene expression [17,21]. The antiestrogens tamoxifen and fulvestrant inhibit estradiol binding to ER $\alpha$. Binding of the selective estrogen receptor modulator (SERM) tamoxifen to ER $\alpha$ leads to inhibition of coactivator recruitment and target gene expression [33]. Binding of the selective estrogen receptor downregulator (SERD) fulvestrant results in altered ligand binding domain structure, decreased ER $\alpha$ dimerization, and increased proteasomal degradation [34]. Aromatase inhibitors (AI) inhibit estrogen action in the breast by decreasing the conversion of the androgens testosterone and androstenedione to estrogens in peripheral tissues and breast stromal cells by inhibiting the enzyme activity of aromatase (CYP179A1) [35]. Estradiol has higher affinity for ER $\alpha$ than estrone [16] and these two estrogens are interconverted

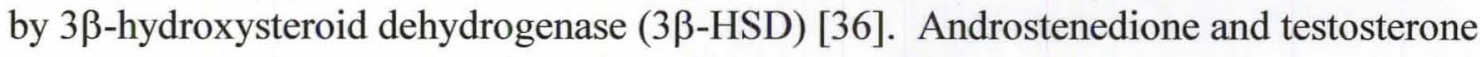
can also be interconverted by $3 \beta-\mathrm{HSD}$ (not pictured) [36]. 


\section{BREAST CANCER TREATMENT}

Since the early 1930s when estrogen was first shown to promote breast cancer formation in mice [37], targeting estrogen signaling has been of interest in the treatment and prevention of breast cancer. In 1971, Jensen demonstrated that breast cancers with higher ER content exhibited a greater response to endocrine ablation than ER- cancers [38]. Studies such as these paved the way to the use of modern antiestrogen therapies to target the proliferative action of estrogen in breast cancer. Selective estrogen receptor modulators (SERMs) such as tamoxifen (TAM) and raloxifene (RAL) exert antiproliferative effects on breast cancer by competing with estrogens for binding to $\mathrm{ER} \alpha$ (Figure 1). First developed as the contraceptive ICI 46,474 [39,40], tamoxifen acts as a cell type-specific agonist/antagonist of the estrogen receptor (agonist in uterus and antagonist in breast) [33]. Binding of 4-hydroxytamoxifen, the active metabolite of tamoxifen, to the ligand binding domain (LBD) of ER $\alpha$ results in a repositioning of $E R \alpha$ helix 12 to cover the hydrophobic coactivator binding groove, preventing binding with coactivators [41] and resulting in an antagonist function in the breast. In other tissues, the increased expression of coactivators such as SRC-1 [42] or the presence of other tissue-specific pathways, as well as promoter-specific functions, allows tamoxifen to function as an agonist $[43,44]$. Treatment with tamoxifen leads to inhibition of cell cycle progression and induction of apoptosis in breast cancer cells $[45,46]$. The agonist activity of tamoxifen in the uterus leads to an increased risk of endometrial cancer [47]. Tamoxifen has greatly increased the survival rate of breast cancer patients since its initial 
FDA approval in 1977 for the treatment of metastatic breast cancer, resulting in a $32 \%$ reduction in annual death rate [48-50].

Another method of targeting the estrogen receptor is through Selective Estrogen Receptor Downregulators (SERD) such as the pure antiestrogen fulvestrant (ICI 182,780), which is a steroidal $7 \alpha$-alkylsulphinyl analogue of estradiol (Figure 1) [34]. Binding of fulvestrant to $\mathrm{ER} \alpha$ results in increased $\mathrm{ER} \alpha$ turnover by the $26 \mathrm{~S}$ proteasome [51]. Reducing the level of ER $\alpha$ blocks estrogen action on target genes in breast cancer [34].

Other treatment options for breast cancer patients with ER $\alpha+$ tumors include the use of aromatase inhibitors (AIs) [35], first proposed in 1973 [52]. In postmenopausal women, the ovaries no longer produce estrogens and progestins. Instead, estrogens are produced via the conversion of the androgens androstenedione and testosterone present in circulation from the adrenals by aromatase located in peripheral tissues, including stromal cells in breast tissue, to estrogens: estrone and estradiol, respectively by the enzyme aromatase (CYP19A1) (Figure 1) [36,53,54]. Aromatase inhibitors such as exemestane, letrozole, and anastrozole work by inhibiting CYP19A1 to stop the production of estrogens. In a five year study of 8,010 women, treatment with letrozole resulted in improved disease-free survival, overall survival, and time to distant recurrence for postmenopausal women, as compared to treatment with tamoxifen or a sequence of tamoxifen and letrozole [55], highlighting the clinical efficacy of aromatase inhibitors as a treatment of choice in postmenopausal women. 


\section{MECHANISMS OF ENDOCRINE RESISTANCE}

Despite the initial efficacy of antiestrogen treatment, approximately $40 \%$ of patients relapse and die from metastatic disease because the cancer cells become endocrine-resistant [56]. This sequela is called acquired endocrine resistance since patients first respond and then relapse. Five years of tamoxifen therapy results in a $33.2 \%$ probability of recurrence at 15 years [49]. Many potential causes of endocrine resistance have been identified. Altered expression of ER $\alpha$ itself was identified to account for only $15-20 \%$ of acquired endocrine resistance [57], demonstrating that mechanisms other than direct loss of the antiestrogen target contribute to endocrineresistant phenotypes. A variant form of $\mathrm{ER} \alpha, \mathrm{ER} \alpha 36$, has also been implicated in tamoxifen resistance, as ER $\alpha+$ women receiving tamoxifen treatment who also had increased expression of ER $\alpha 36$ had shorter disease-free survival [58].

\section{Altered coregulator expression}

A proper coactivator/corepressor ratio is necessary for basal estrogen-regulated gene expression. Increased coactivator or reduced corepressor expression or altered function has been implicated in endocrine resistance. Overexpression of coactivator SRC-3 has been identified in breast cancer and is correlated with reduced responsiveness to tamoxifen therapy $[59,60]$. In endocrine-resistant LY2 breast cancer cells, treatment with tamoxifen increased nuclear colocalization of both SRC-1 and SRC-3 coactivators with $\mathrm{ER} \alpha$, indicating tamoxifen instead acts as an $\mathrm{ER} \alpha$ agonist rather than antagonist in these cells [61]. Further, tamoxifen treatment itself can increase the expression of SRC-1 and SRC-3, promoting increased endocrine resistance [62]. The corepressor NCoR is 
downregulated in a mouse model of progression to tamoxifen resistance [63]. Reduced NCoR expression was also found to be associated with shorter relapse-free survival of patients treated with tamoxifen [64]. In contrast, no correlation between the NCoR2/SMRT corepressor and recurrence was found through immunohistochemical (IHC) staining of breast tumors from tamoxifen-treated patients [65]. SMRT expression was instead correlated with shortened time to recurrence in untreated patients, suggesting a different role for this corepressor, such as in inhibition of the activity of transcription factors other than ER $\alpha[65]$.

\section{Increased kinase signaling}

Nongenomic ER signaling can occur via plasma membrane (PM)-associated forms of ER which, when activated by ligand, stimulate rapid cellular responses through subsequent effects on downstream kinase activation [66-70]. ER $\alpha$ localization to the PM may be mediated through palmitoylation at cysteine 447 leading to interaction with caveolin-1 at lipid rafts (caveolae) in a ligand-independent manner [71,72]. Increased localization of ER $\alpha$ and a shorter splice variant ER $\alpha 46$ in lipid rafts occurs with HER2 overexpression, and ER $\alpha$ colocalizes with HER2 in MCF-7 cells [73]. In addition to HER2, PM-associated ER $\alpha$ interacts with other growth factor receptors/receptor tyrosine kinases (RTKs) such as EGFR and IGFR [74,75]. Elevated EGFR and HER2 expression has been reported in tamoxifen-resistant breast cancer cell lines, leading to downstream activation of the ERK pathway [76]. Increased expression of the coactivator SRC-3 and HER2 enabled tamoxifen to switch to an agonistic effect in breast cancer cells, recruiting ER $\alpha$ coactivators and stimulating cell proliferation [77]. Activation of EGFR and HER2 
signaling can also promote cell proliferation independent of the involvement of ER, creating a mechanism for cells to bypass the normal inhibitory effects of tamoxifen and other antiestrogens [78].

Kinases other than RTKs also have roles in progression to tamoxifen resistance. Protein kinase $\mathrm{C}$ (PKC) activation is known to increase cell proliferation [79]. Elevated PKC $\alpha$ expression was detected in breast cancer cell lines that have undergone progression to tamoxifen or fulvestrant resistance, and treatment with a PKC inhibitor reduced cell proliferation [80]. Knockdown of PKC $\alpha$ expression with shRNA was able to restore cell sensitivity to tamoxifen but not fulvestrant, suggesting the involvement of other pathways [80]. Ligand-activated ER can interact with PI3K to activate Akt and lead to subsequent signaling [81]. Reciprocally, Akt can phosphorylate ER $\alpha$ at serine 167 to lead to $\mathrm{ER} \alpha$ activation independent of ligand binding [82]. Tamoxifen can also activate Akt through phosphorylation at Serine 473 in ER+ breast cancer, which may play a further role in the contribution of Akt to tamoxifen resistance [83]. Inhibition of Akt with a PI3K inhibitor increased apoptosis in response to tamoxifen treatment [83].

\section{Altered transcription factor expression/signaling}

The nuclear receptor superfamily includes ligand/steroid activated receptors, such as ER, that act as transcription factors to modulate target gene expression. Alteration of nuclear receptor signaling has been implicated in development of many types of cancer, including breast cancer [84]. Muscat et al. recently demonstrated expression of nuclear

receptors COUP-TFII (gene name $N R 2 F 2$ ), thyroid hormone receptor $\beta(T R \beta)$, 
peroxisome proliferator-activated receptor $\gamma(P P A R \gamma)$, and mineralocorticoid receptor $(M R)$ to be predictive of metastasis-free survival in tamoxifen-treated patients [85].

Activation of the inflammatory NFKB pathway leads to increased expression of genes involved in cellular processes such as proliferation, apoptosis resistance, and other oncogenic functions [86]. Several groups have reported an association between increased NFkB pathway activation and tamoxifen resistance [87-92]. Elevated expression of one or more of the five subunits that comprise the NFKB transcription factor homodimer or heterodimer has also been reported, including upregulation of RelA/p65 in tamoxifenresistant breast cancer cell lines LCC9 and MCF-7/RR [87,88] and increased RelA/p65 and NFKB1/p50 in a estrogen-independent breast cancer cell line LCC1 [93].

Increased AP-1 DNA binding was observed in a fulvestrant-resistant breast cancer cell line [94] as well as in a set of ER $\alpha+$ tamoxifen-resistant human breast tumor samples [20], suggesting AP-1 may provide an alternative pathway for transcription in the presence of antiestrogens. Increased activity of the c-Jun $\mathrm{NH}_{2}$-terminal kinase (JNK), which phosphorylates and activates AP-1, was also identified in tamoxifen-resistant human breast tumors [20]. In addition, overexpression of c-Jun, a member of the AP-1 heterodimer, rendered MCF-7 cells unresponsive to endocrine therapy [95].

\section{Dysregulated microRNA expression}

MicroRNAs (miRNAs) are short, noncoding RNAs of 19-25 nucleotides in length that downregulate gene expression post-transcriptionally via binding to the 3'UTR of target mRNAs in a sequence-dependent manner leading to degradation or inhibition of

translation. Altered expression of miRNAs in cancer has been widely reported, with 
downstream targets in oncogenic systems such as apoptosis, proliferation, cell differentiation, angiogenesis, invasion, metastasis, and DNA repair [96]. Several miRNAs have been identified as targeting the expression of ER $\alpha$, including miR-206 [97] and miR-221/222 [98]. MiR-221/222 was significantly upregulated in tamoxifenresistant breast cancer cell lines and in HER2/neu+ human breast tumor samples [99]. Overexpression of miR-221/222 decreased the sensitivity of cells to treatment with tamoxifen [98]. Besides targeting ER $\alpha$, miR-221/222 also targets the cell cycle inhibitor $\mathrm{p} 27^{\mathrm{Kip} 1}$ [99]. A reduction in $\mathrm{p} 27^{\mathrm{Kip} 1}$ also resulted in decreased growth inhibition by tamoxifen treatment in breast cancer cells [99].

In addition to these studies, microarray analysis identified twelve miRNAs that were differentially expressed between MCF-7 tamoxifen-sensitive and LY2 tamoxifenresistant breast cancer cells [100]. Identified targets of these miRNAs had known roles in apoptosis (PDCD4 and BCL2), estradiol metabolism (CYP1B1), epithelial to mesenchymal transition (ZEB1), and growth factor signaling (ERBB3). ER $\alpha$ itself was also identified as a target in this screen [100].

\section{Altered DNA methylation}

Changes in gene promoter methylation can also occur upon progression to antiestrogen resistance, either through activation of the expression of genes which promote proliferation through promoter hypomethylation (more common) or via downregulation of tumor suppressive genes through hypermethylation (less common) [101]. In one study, tamoxifen-resistant cell proliferation remained ER $\alpha$-driven, while fulvestrant-resistant cell proliferation was driven by mechanisms other than ER $\alpha$ [101]. 
Many more changes in promoter methylation were found in the fulvestrant resistant cells, where altered methylation was identified for 72 genes compared to only 10 genes in tamoxifen-resistant cells [101].

\section{CONCLUSIONS}

Most breast tumors are initially driven by estrogens through activation of ER $\alpha$. Targeting ER $\alpha$ by SERMs, e.g., tamoxifen, and SERDs, i.e., fulvestrant, and AIs, e.g., letrozole, have been successful in increasing the overall survival of breast cancer patients in the last $25-30$ yrs $[33,48,102]$. Unfortunately, $40 \%$ of patients relapse on endocrine therapies [56] and understanding the mechanism by which tumors escape inhibition by AIs, SERMs, and SERDs and re-establishing endocrine-sensitivity is key to enhancing overall survival. Although many mechanisms have been identified as contributing to the endocrine-resistant phenotype, the field is currently lacking clinically relevant biomarkers and functional drug targets. More work is necessary to fully elucidate the complexity of interactions between the current observations and translate these findings into a unified model of the endocrine-resistance. 


\section{CHAPTER II - MULTIPLE ROLES OF COUP-TFII IN CANCER INITIATION AND PROGRESSION}

The text of this chapter was published in Journal of Molecular Endocrinology 49(3): R135-48, 2012. doi:10.1530/JME-12-0144

\section{INTRODUCTION}

Steroid hormones and nuclear receptor ligands play critical roles in cancer initiation and progression and their antagonists have proven efficacy in the treatment and prevention of cancers. This is most notable in breast and prostate cancers and the use of all-trans retinoic acid for acute promyelocytic leukemia [103,104]. Steroid/nuclear receptors act as ligand-activated transcription factors to either positively or negatively regulate gene expression $[84,105]$. Activation of nuclear receptors occurs through binding a variety of ligands including hormones and vitamins/retinoids. Nuclear receptors (NR) have physiological roles to modulate gene expression during development and growth. As alteration of basal gene expression leads to many pathogenic outcomes including cancer, maintenance of normal gene expression by nuclear receptors is vital. One such critical nuclear receptor is chicken ovalbumin upstream promoter transcription factor II (COUP-TFII). From the time of the identification of the COUP-TF family in 1986 [106], the many functions of COUP-TFs have continued to be explored. The role of 
COUP-TFII in cancer is widely debated with evidence linking COUP-TFII to both tumor suppressive and oncogenic functions. This review will explore both the regulation and function of COUP-TFII and its connections to cancer.

\section{COUP-TFI AND COUP-TFII}

The COUP-TF family consists of two highly homologous subtypes, COUP-TFI and COUP-TFII, located on human chromosomes 5 and 15, respectively (Figure 2). COUP-TFs have been previously reviewed $[107,108]$, but not in the specific context of separating COUP-TFI and COUP-TFII in cancer. COUP-TFs are ancient NRs and are located close to retinoid $\mathrm{X}$ receptors (RXRs) in the evolutionary tree $[109,110]$. As evolutionarily conserved transcription factors, COUP-TFs have major roles in development. The importance of COUP-TFII expression is evidenced by studies in knockout mice [111]. Homozygous mutation of COUP-TFII leads to embryonic lethality due to impaired angiogenesis and heart defects, resulting in hemorrhage and edema. These effects may in part be explained by the reduction in angiopoietin-1 expression in COUP-TFII-null mice [111]. Other important embryonic roles for COUP-TFII include regulation of limb growth and muscle development [112]. COUP-TFII-null mice display a reduction in expression of $L b x l$, a protein required for proper muscle precursor cell migration, and in myogenin, which is necessary for muscle cell differentiation $[112,113]$.

Based on the high sequence identity in their DNA binding domains DBDs (Figure 2), we anticipate that COUP-TFI and COUP-TFII regulate the same genes. However, this has not been empirically tested and it is worth noting that the $\mathrm{N}$-terminus is divergent (Figure 2) and immunoprecipitation studies indicate differences in proteins interacting with COUP-TFI [114] and COUP-TFII [115], although, again, this has not been 


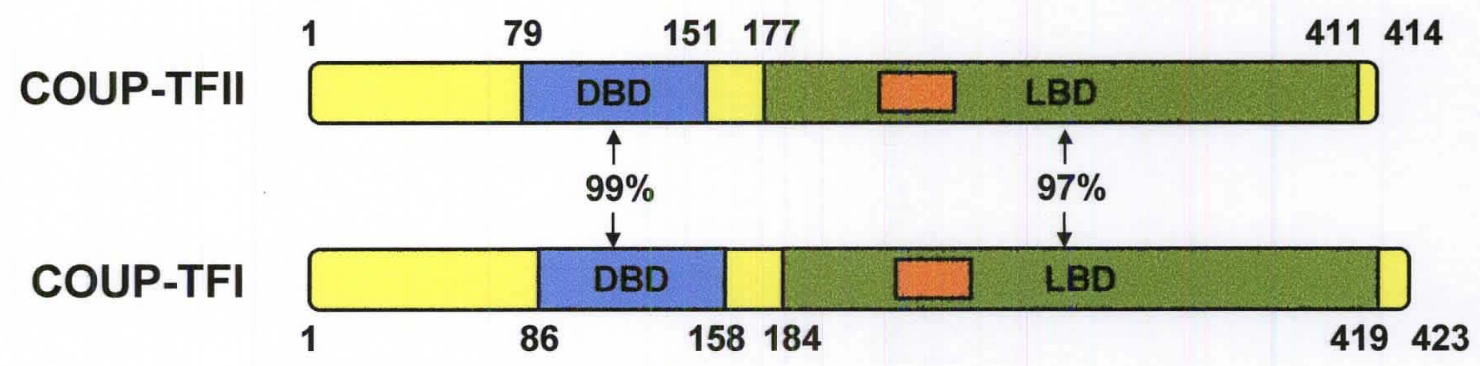

Figure 2. Comparison of COUP-TFI and COUP-TFII protein homology.

COUP-TFI (NP_005645.1) and COUP-TFII (NP_066285.1) amino acid sequences were obtained from the National Center for Biotechnology Information. The COUP-TF DNA binding domains (DBD) and ligand binding domains (LBD) share $99 \%$ and $97 \%$ amino acid homology, respectively. Positions noted as important in coactivator recognition are shown in orange. 
systematically studied in cells in which both are expressed. COUP-TFI and COUP-TFII may have divergent functions in certain contexts as well. Differences in COUP-TFI and COUP-TFII function in breast cancer endocrine sensitivity, for example, have also been identified [116]. This review will focus specifically on COUP-TFII.

\section{COUP-TFII REGULATION OF GENE EXPRESSION}

\section{Mechanisms of regulation}

COUP-TFII can activate or repress gene expression in both a tissue-specific and gene-specific manner through mechanisms involving direct binding to DNA response elements or binding to other transcription factors. Through binding to 5'-AGGTCA-3' direct repeats (DR) with variable spacing [117], COUP-TFII modulates the expression of target genes. Specific genes upon which COUP-TFII activates transcription include retinoic acid receptor $\beta 2$ (RAR $\beta 2, \quad R A R B 2)$ [115,118], phosphoenolpyruvate carboxykinase (PEPCK, PCK1) [119], NGFI-A [120,121], and cholesterol 7ahydroxylase (CYP7A1) [122]. COUP-TFII action may be potentiated by interaction with coactivators such as steroid receptor coactivator family members SRC-1/NCOA1, SRC2/NCOA2, and SRC-3/NCOA3 [120,121], as well as PGC1 $\alpha$ [120], p300/CBP [121], orphan receptor coactivator (ORCA) [123], and nucleolin [115]. DNA binding of COUPTFII can promote the binding of a second transcription factor, further activating gene transcription. This occurs for both the PEPCK and CYP7A1 genes, where COUP-TFII binding to the promoter recruits binding of glucocorticoid receptor (GR) to enhance gene expression $[119,124]$. COUP-TFII can also bind to Sp1 sites to cooperatively activate 
gene expression, as was reported for regulation of $0 t x 2$ expression during morphogenesis in the mouse eye [125].

Alternatively, binding of COUP-TFII to DRs may result in repression of gene expression. In the mechanism of "active repression," COUP-TFII binding results in recruitment of corepressors, i.e., nuclear corepressor (NCoR) [126] and silencing mediator of retinoid and thyroid receptors (SMRT) $[127,128]$, resulting in repressed chromatin structure and a corresponding blockade of target gene transcriptional activation. COUP-TFII interaction with SMRT represses PPAR $\gamma 1$ and PPAR $\gamma 2$ expression to suppress adipogenesis [128]. Repression of the human oxytocin promoter by COUP-TFII binding has also been reported [129]. COUP-TFII represses Pax2 expression in the retina via binding to a DR1 site (TGTTCACAGTCCA) [125].

Through an alternative mechanism of transrepression, COUP-TFII can interact with other nuclear receptors and transcription factors to inhibit their normal transcriptional activity. Examples of this include inhibition of ER- and GR-induced gene expression in a gene-specific manner $[119,130]$. COUP-TFII can also repress AP-1 signaling through interaction with c-Jun [131]. Interaction of COUP-TFII with Runx2 inhibits osteoblast differentiation via blocking Runx 2 binding to the osteocalcin promoter [132]. Other mechanisms of repression involve the modulation of ER, RXR, PPAR, and VDR activity by competing for DNA response element-binding or heterodimerization with the class II heterodimeric partner RXR [133].

Ingenuity Pathway Analysis 
As summarized here, COUP-TFII regulates the expression of diverse gene targets. Table 1 contains a list of known COUP-TFII targets as identified using Ingenuity Pathway Analysis (IPA; Ingenuity Systems, www.ingenuity.com). These targets are also displayed in Figure 3. COUP-TFII has varying effects on expression of other nuclear receptors and transcription factors. COUP-TFII increased the expression of HNF-1 $\alpha$ [134], HNF-1 $\beta$ [135], HNF-4 $\alpha$ [136], and RAR $\beta$ [115,118,137], while it decreased the expression of Oct4 [138,139], Dax1 [140], and PPAR $\alpha$ [141]. As previously described, COUP-TFII has well known functions in repressing the transcriptional activity of other nuclear receptors and transcription factors. Although COUP-TFII increases HNF-4 expression, other reports highlight the repression of HNF-4 function by COUP-TFII. Specifically, COUP-TFII decreases transcriptional activation of $A L D H 2$ [142] and retinol binding protein 2 (RBP2) [143] by HNF-4. The HNF-4 activation of hepatic lipase is suppressed by COUP-TFII [144], while lipoprotein lipase expression is induced by COUP-TFII synergistically with PPAR $\gamma$ [145]; part of the many of reported functions of COUP-TFII in the cholesterol processing pathway. A similar response occurs for apolipoproteins A-I, A-IV, and C-III, where COUP-TFII represses the RXR $\alpha$-mediated expression of APOA-I [135,146,147] and HNF4-mediated expression of APOA-IV $[148,149]$ and APOC-III $[134,135,150,151]$. HNF-4 and COUP-TFII binding to the sex hormone binding globulin $(S h b g)$ promoter was reported in murine Sertoli cells [152]. SHBG expression is increased by HNF-4 and suppressed by COUP-TF in HepG2 hepatoblastoma cells [153]. Decreased SHBG expression is indicative of metabolic syndrome and may result in increased plasma androgen and estrogen levels, though the precise connection of COUP-TFII to these phenotypes has not been investigated [154]. 
Although COUP-TFII is classically known for its role in transrepression, COUPTFII may also enhance the effect of a second nuclear receptor. Induction of cytochrome P450 family members cholesterol 7a-hydroxylase CYP7A1 [122] and aldosterone synthase CYP11B2 $[155,156]$ by COUP-TFII was reported, with COUP-TFII and HNF-4 acting to synergistically activate CYP7A1 [122]. CYP7A1 catalyzes the first step in the conversion of cholesterol to bile acid [122], while CYP11B2 catalyzes the final steps of aldosterone synthesis [155], implying that COUP-TFII transcriptional activation would increase the production of bile acid and aldosterone.

As shown in Table 1 and Figure 3, COUP-TFII opposes PPAR $\gamma /$ RXR activation of PEPCK transcription in predadipocytes/fibroblasts, a result that was proposed to suppress adipogenesis [157]. COUP-TF also inhibited 9-cis retinoic acid/RXR-induced activation of the lactotransferrin promoter in transiently transfected ZR-75-1 and Hs578T breast cancer cells apparently by competing for DNA binding to a composite RARE/ERE in the gene promoter [158]. Concurrent binding of COUP-TFII and NF-Y to the hemoglobin epsilon promoter leads to a repression of gene expression [159]. In addition to the targets identified by IPA, COUP-TF was reported to play a dual regulatory role in the transcriptional regulation of the mitochondrial HMG-CoA synthase gene: alone COUP-TFI stimulated reporter gene activity from the HMG-CoA synthase promoter in transiently transfected HepG2 human hepatoma and rat Leydig tumor R2C cells, but it inhibited PPAR $\alpha$-stimulated transcriptional activity by competing for the same DNA binding site [160].

Some of the IPA-identified COUP-TFII target gene relationships and mechanisms remain to be fully elucidated. In a study of the transcriptional regulation of murine 


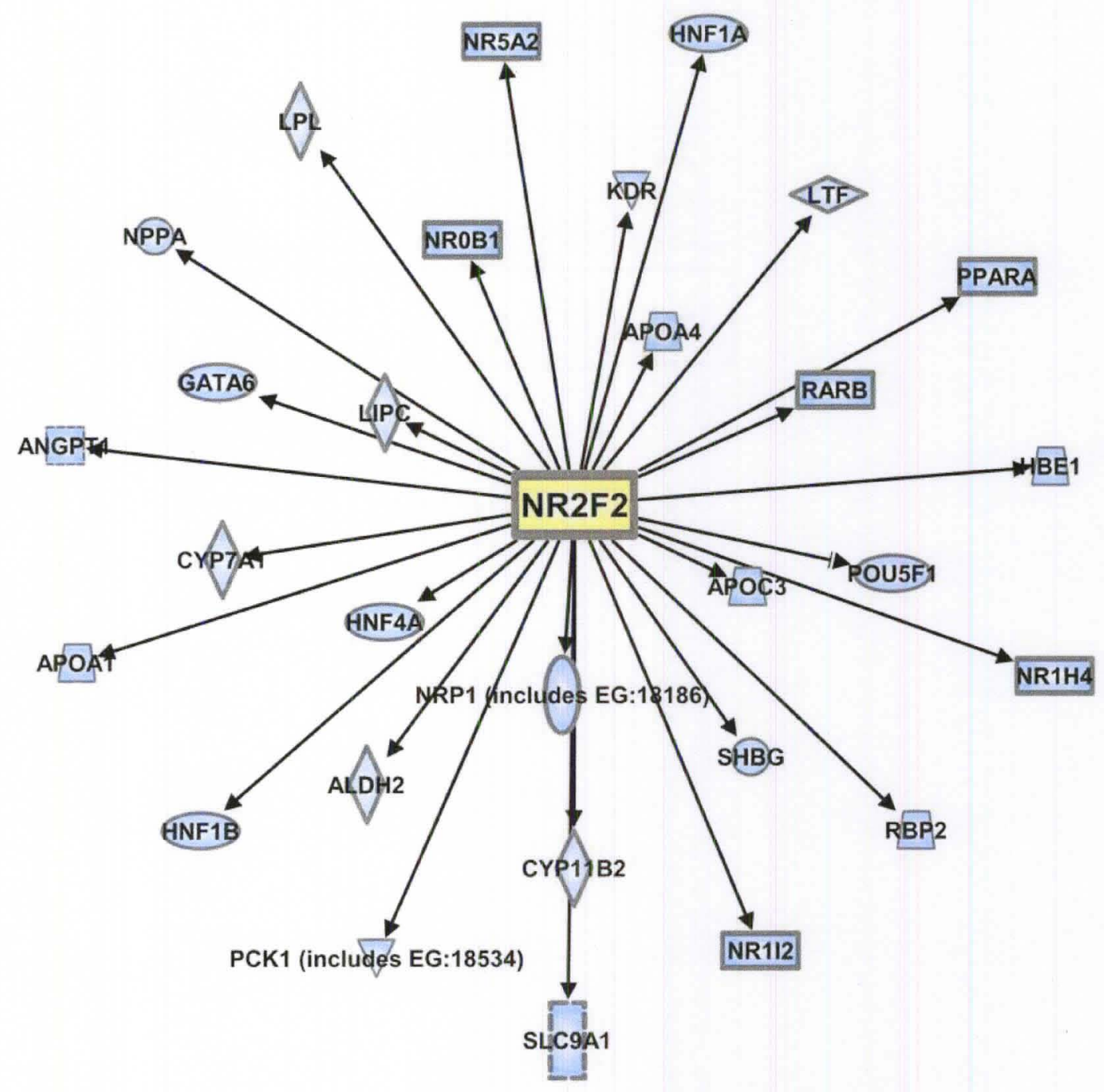

Figure 3. COUP-TFII (NR2F2) target genes.

COUP-TFII has been reported to modulate the expression of a variety of target genes both positively and negatively. A list of COUP-TFII target genes and corresponding network pathway were generated using Ingenuity Pathway Analysis (IPA; Ingenuity Systems, www.ingenuity.com). 
Table 1. List of COUP-TFII-regulated genes identified by Ingenuity Pathway Analysis

\begin{tabular}{|c|c|c|c|c|c|}
\hline $\begin{array}{c}\text { Gene } \\
\text { (protein) }\end{array}$ & Name & Location & Family & $\begin{array}{l}\text { Regulation } \\
\text { by COUP- } \\
\text { TFII }\end{array}$ & Reference \\
\hline ALDH2 & $\begin{array}{l}\text { Aldehyde dehydrogenase } 2 \text { family } \\
\text { (mitochondrial) }\end{array}$ & Cytoplasm & Enzyme & Decrease & [142] \\
\hline ANGPT1 & Angiopoietin 1 & $\begin{array}{l}\text { Extracellular } \\
\text { Space }\end{array}$ & Growth factor & Increase & [111] \\
\hline APOA1 & Apolipoprotein A-I & $\begin{array}{l}\text { Extracellular } \\
\text { Space }\end{array}$ & Transporter & Decrease & {$[135,146,147]$} \\
\hline APOA4 & Apolipoprotein A-IV & $\begin{array}{c}\text { Extracellular } \\
\text { Space }\end{array}$ & Transporter & Decrease & {$[148,149]$} \\
\hline APOC3 & Apolipoprotein C-III & $\begin{array}{l}\text { Extracellular } \\
\text { Space }\end{array}$ & Transporter & Decrease & $\begin{array}{l}{[134,135,150,} \\
151]\end{array}$ \\
\hline CYP11B2 & $\begin{array}{c}\text { Aldosterone synthase, cytochrome } \\
\text { P450, family } 11 \text {, subfamily B, } \\
\text { polypeptide } 2\end{array}$ & Cytoplasm & Enzyme & Increase & {$[155,156]$} \\
\hline CYP7A1 & $\begin{array}{c}\text { Cholesterol } 7 \text { alpha-hydroxylase, } \\
\text { cytochrome P450, family } 7 \\
\text { subfamily A, polypeptide } 1 \\
\end{array}$ & Cytoplasm & Enzyme & Increase & [122] \\
\hline GATA6 & GATA binding protein 6 & Nucleus & $\begin{array}{l}\text { Transcription } \\
\text { regulator }\end{array}$ & $\begin{array}{l}\text { Decrease } \\
\text { suggested }\end{array}$ & [161] \\
\hline HBE1 & Hemoglobin, epsilon 1 & Cytoplasm & Transporter & Decrease & {$[159,162]$} \\
\hline HNF1A & HNF1 homeobox A & Nucleus & $\begin{array}{c}\text { Transcription } \\
\text { regulator }\end{array}$ & Increase & {$[134,161]$} \\
\hline HNF1B & HNF1 homeobox B & Nucleus & $\begin{array}{l}\text { Transcription } \\
\text { regulator }\end{array}$ & Increase & [135] \\
\hline HNF4A & Hepatocyte nuclear factor 4 , alpha & Nucleus & $\begin{array}{c}\text { Transcription } \\
\text { regulator }\end{array}$ & Increase & {$[136]$} \\
\hline KDR & $\begin{array}{l}\text { VEGFR-2; kinase insert domain } \\
\text { receptor (a type III receptor } \\
\text { tyrosine kinase) }\end{array}$ & $\begin{array}{l}\text { Plasma } \\
\text { Membrane }\end{array}$ & Kinase & Decrease & [163] \\
\hline LIPC & Lipase, hepatic & $\begin{array}{l}\text { Extracellular } \\
\text { Space }\end{array}$ & Enzyme & Decrease & [144] \\
\hline LPL & Lipoprotein lipase & Cytoplasm & Enzyme & Increase & [145] \\
\hline LTF & Lactotransferrin & $\begin{array}{l}\text { Extracellular } \\
\text { Space }\end{array}$ & Peptidase & Decrease & [158] \\
\hline NPPA & Natriuretic peptide A & $\begin{array}{c}\text { Extracellular } \\
\text { Space }\end{array}$ & Other & Increase & [164] \\
\hline NR0B1 & $\begin{array}{c}\text { Dax1, nuclear receptor subfamily } \\
0 \text {, group B, member } 1\end{array}$ & Nucleus & $\begin{array}{l}\text { Ligand-dependent } \\
\text { nuclear receptor }\end{array}$ & Decrease & [140] \\
\hline $\mathrm{NR} 1 \mathrm{H} 4$ & $\begin{array}{c}\text { FXR, nuclear receptor subfamily } 1 \text {, } \\
\text { group } \mathrm{H} \text {, member } 4\end{array}$ & Nucleus & $\begin{array}{l}\text { Ligand-dependent } \\
\text { nuclear receptor }\end{array}$ & Unknown & [161] \\
\hline NR112 & $\begin{array}{c}\text { PXR, nuclear receptor subfamily } 1, \\
\text { group I, member } 2\end{array}$ & Nucleus & $\begin{array}{l}\text { Ligand-dependent } \\
\text { nuclear receptor }\end{array}$ & Unknown & [161] \\
\hline NR5A2 & $\begin{array}{c}\text { LRH-1, nuclear receptor subfamily } \\
5 \text {, group A, member } 2\end{array}$ & Nucleus & $\begin{array}{l}\text { Ligand-dependent } \\
\text { nuclear receptor }\end{array}$ & Unknown & [161] \\
\hline NRP1 & Neuropilin 1 & $\begin{array}{c}\text { Plasma } \\
\text { Membrane }\end{array}$ & $\begin{array}{c}\text { Transmembrane } \\
\text { receptor }\end{array}$ & Decrease & [163] \\
\hline PCK1 & $\begin{array}{c}\text { Phosphoenolpyruvate } \\
\text { carboxykinase } 1 \text { (soluble) }\end{array}$ & Cytoplasm & Kinase & Decrease & [157] \\
\hline POU5F1 & Oct 4 ; POU class 5 homeobox 1 & Nucleus & $\begin{array}{c}\text { Transcription } \\
\text { regulator }\end{array}$ & Decrease & {$[138,139]$} \\
\hline PPARA & $\begin{array}{c}\text { Peroxisome proliferator-activated } \\
\text { receptor alpha }\end{array}$ & Nucleus & $\begin{array}{l}\text { Ligand-dependent } \\
\text { nuclear receptor }\end{array}$ & Decrease & {$[141]$} \\
\hline RARB & Retinoic acid receptor, beta & Nucleus & $\begin{array}{l}\text { Ligand-dependent } \\
\text { nuclear receptor }\end{array}$ & Increase & $\begin{array}{l}{[115,118,137,} \\
143]\end{array}$ \\
\hline RBP2 & Retinol binding protein 2 , cellular & Cytoplasm & Transporter & Decrease & [143] \\
\hline SHBG & Sex hormone-binding globulin & $\begin{array}{l}\text { Extracellular } \\
\text { Space }\end{array}$ & Other & Decrease & {$[152,153]$} \\
\hline SLC9A1 & $\begin{array}{c}\text { Solute carrier family } 9 \text {, subfamily } A \\
\text { (NHE1, cation proton antiporter } 1 \text { ), } \\
\text { member } 1\end{array}$ & $\begin{array}{l}\text { Plasma } \\
\text { Membrane }\end{array}$ & Ion channel & Increase & [165] \\
\hline
\end{tabular}


hepatic development, COUP-TFII occupancy of GATA-6, FXR, PXR, and LRH-1 promoters, as determined by chromatin immunoprecipitation (ChIP) assay, was reported during the postnatal period [161]. While an inhibitory relationship was suggested for the effect of COUP-TFII on GATA-6, the effect on FXR, PXR, and $L R H-1$ expression is not yet known [161]. Several other target genes have been identified that highlight the critical function of COUP-TFII in the vascular system. These include an increase in angiopoietin 1 [111] and natriuretic peptide A [164] by COUP-TFII, and a decrease in VEGFR-2 and neuropilin 1 [163]. COUP-TFII enhances expression of the NHE1 solute exchanger $[165,166]$.

In summary, as indicated by the IPA (Figure 3) and consistent with previous reports, COUP-TFII plays a role in many downstream pathways and may either activate or suppress gene expression.

Role in the retinoic acid pathway

COUP-TFs are classified as orphan members of the NR superfamily, because their endogenous ligand(s) is not known. However, Kruse et al demonstrated in silico binding of all-trans (atRA) and 9-cis (9cRA) retinoic acid to the crystal structure of the COUP-TFII LBD [120]. RA released the COUP-TFII LBD from the autorepressed conformation. While the investigators did not directly test binding of all-trans or 9-cis RA to COUP-TFII, they demonstrated that treatment with atRA or 9cRA increased COUP-TFII interaction with the coactivator SRC-3, with an $\mathrm{EC}_{50}$ of $10-30 \mu \mathrm{M}$. In agreement with this data, addition of $20 \mu \mathrm{M}$ atRA or 9cRA led to COUP-TFIl's activation of a NGFI-A-luciferase reporter [120]. Although these concentrations of atRA 
and 9cRA are greater than the physiological concentration of these retinoids, this finding provides novel insight into the ligand binding ability of COUP-TFII. Indeed, the function of this activation can be seen in the regulation of RAR $\beta 2$ by COUP-TFII, as COUP-TFII activation of RAR $\beta 2$ expression is increased with the addition of all-trans retinoic acid $[115,118]$. Treatment of MCF-7 breast cancer cells with atRA also increased COUPTFII-binding to the $R A R B 2$ promoter in a ChIP assay [115]. Retinoic acid induces the expression of COUP-TFII in certain breast cancer cell lines (e.g. T47D and ZR-75) but not others (e.g. MCF-7 and MDA-MB-231) (Figure 4) $[115,167]$. This indicates a potential feed forward loop, as treatment with retinoic acid may increase both the expression and activation of COUP-TFII, with downstream effects on retinoic acid receptor.

\section{REGULATION OF COUP-TFII EXPRESSION}

Tissue-specific regulation in humans

COUP-TFII has a widespread tissue distribution, with detectable expression in every human tissue type examined [168]. The regulation of COUP-TFII expression is tissue and cell-type specific, and can be modulated both transcriptionally and posttranscriptionally (Figure 4). Hyperinsulinemia is a risk for breast cancer $[169,170]$. COUP-TFII expression was repressed by insulin and glucose in the liver and pancreas of C57BL6/J mice and in mouse primary hepatic and pancreatic cell culture [171]. In contrast, we found that insulin treatment had no effect on COUP-TFII expression in 


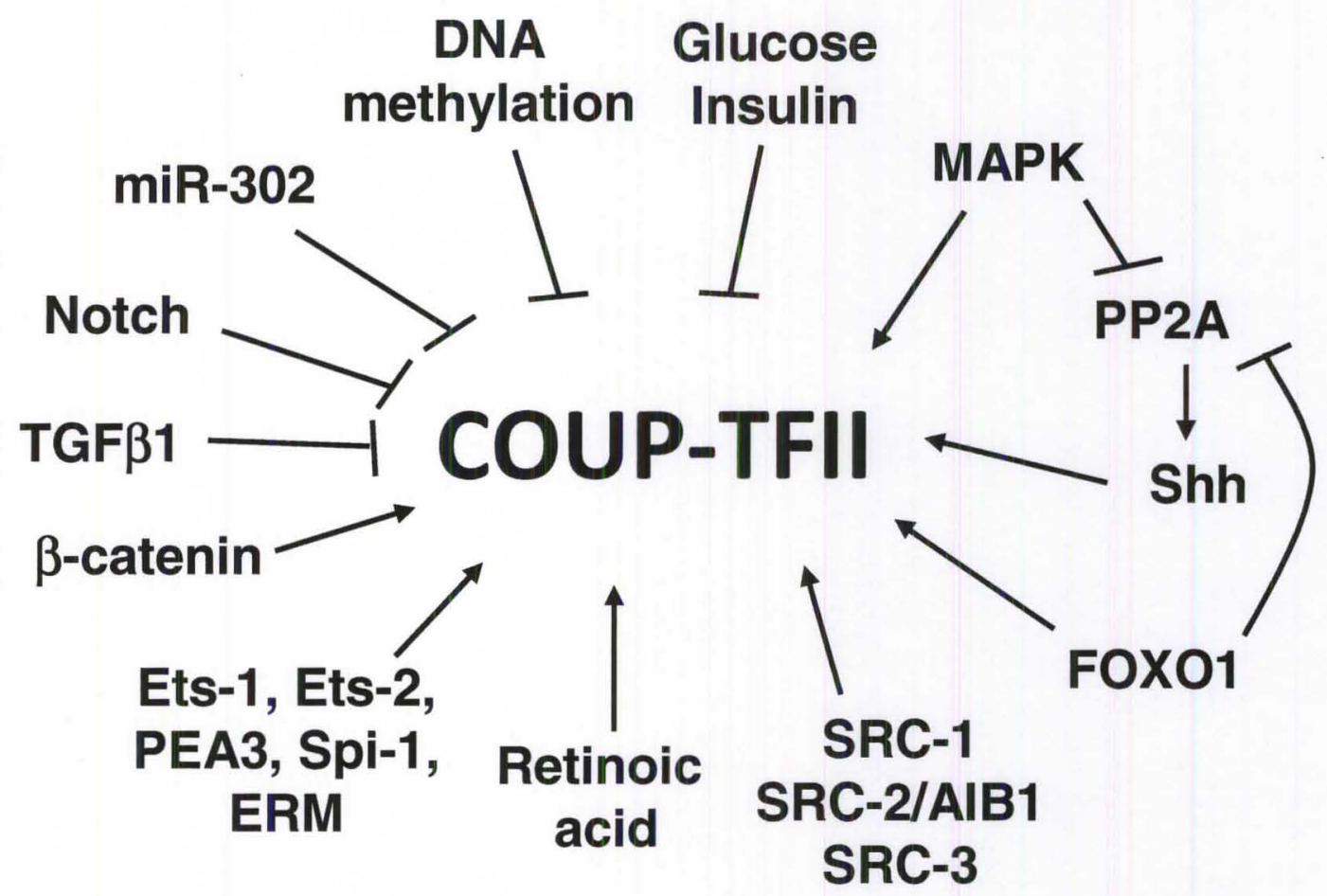

Figure 4. Regulation of COUP-TFII expression.

COUP-TFII expression has been shown to be modulated both transcriptionally and posttranscriptionally by a variety of transcription factors, signaling pathways, and various molecules, as diagramed here. 

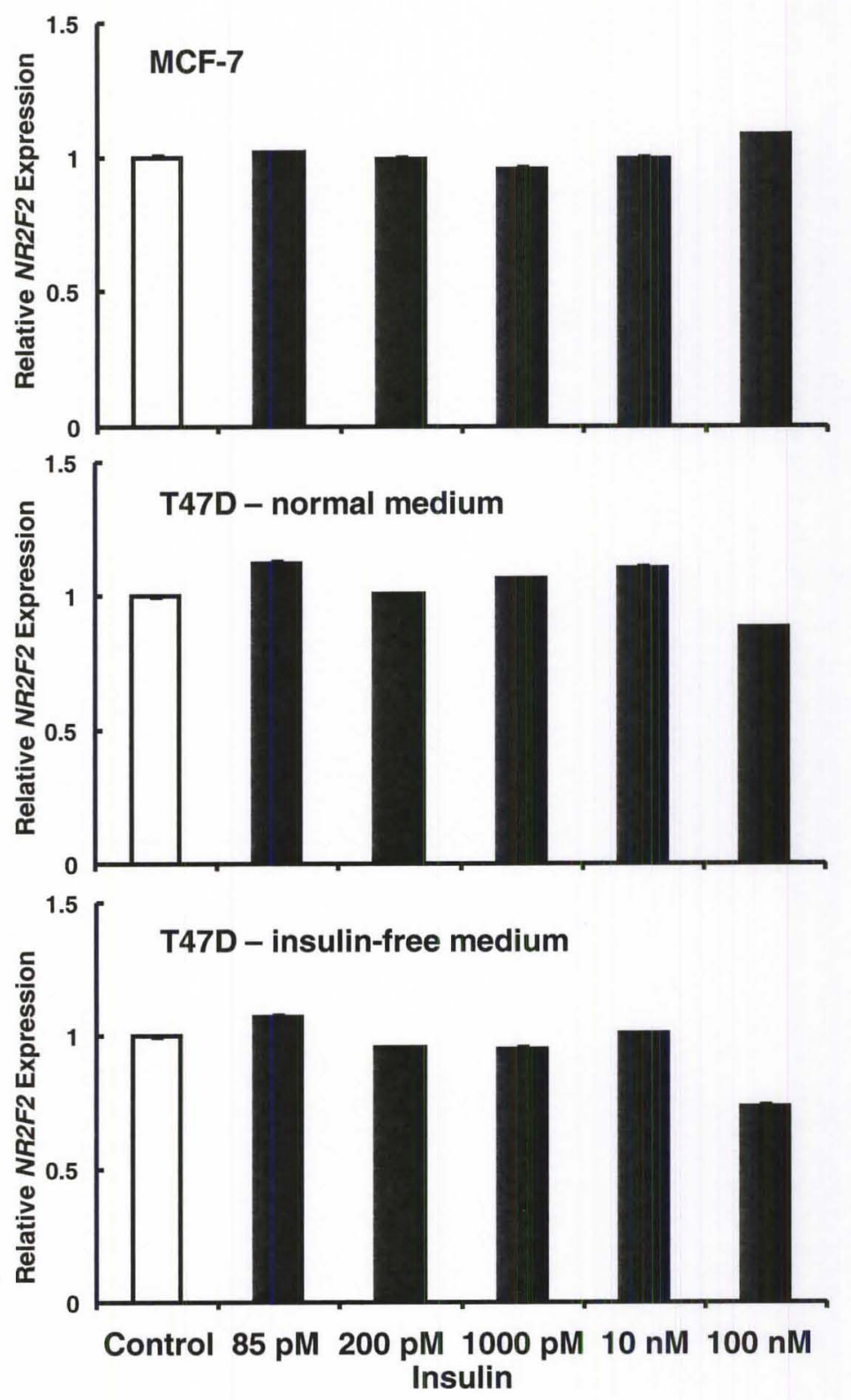

Figure 5. Insulin treatment does not affect COUP-TFII expression in human breast cancer cells.

MCF-7 and T47D human breast cancer cells were grown as in described in [115]. As T47D growth medium contains $6 \mathrm{mg} / \mathrm{ml}$ insulin, T47D cells were either grown in normal medium (with insulin) or in insulin-free medium to determine if this affected outcome. Prior to treatment with insulin, all cells were "starved" in low glucose medium $(5 \mathrm{mM}$ glucose) for $24 \mathrm{~h}[136,171]$. Cells were treated for $6 \mathrm{~h}$ with the indicated concentrations of insulin. QRT-PCR was performed to measure $N R 2 F 2$ expression relative to GAPDH as a reference gene, as described in [115]. Insulin treatment had no statistically significant effect on COUP-TFII expression in these cell lines. 
MCF-7 and T47D breast cancer cells (Figure 5). The lack of alteration in COUP-TFII expression with insulin in breast cancer cells highlights the importance of cell-specific regulation of COUP-TFII expression. There are currently no reports on the effect of insulin on COUP-TFII expression in other cancers.

\section{miRNA regulation}

MicroRNA (miRNA) expression is altered in a variety of conditions and disease states, including cancer, and results in important post-transcriptional regulation of crucial proteins [96]. While 115 miRNAs are predicted to target $N R 2 F 2$ (http://cometa.tigem.it/site/index.php), only one miRNA has been verified. miRNA-302 directly represses COUP-TFII expression in human embryonic stem cells [138]. Regulation of COUP-TFII expression by miRNA has not yet been reported in cancer cells.

\section{DNA methylation}

Methylation at $\mathrm{CpG}$ islands can result in suppression of gene transcription, and is known to be a hallmark of cancer progression. DNA methylation may also occur at intragenic and intergenic sites, as well as at the promoter [172,173]. Specifically, COUPTFII has been found to be methylated in many cancers, including mantle cell lymphoma, acute myeloid leukemia, salivary gland adenoid cystic carcinoma, pancreatic adenocarcinoma, colon cancer, breast cancer ductal carcinoma in situ, as well as a tamoxifen-resistant breast cancer cell line [101,174-179]. NR2F2 gene hypermethylation was associated with a concordant reduction in mRNA expression in mantle cell 
lymphoma, pancreatic cancer, and tamoxifen-resistant breast cancer cells [101,174,177]. Whether this indicates a general trend of reduced COUP-TFII expression due to epigenetic modification across cancer types remains to be seen. Contrary to these reports, high levels of COUP-TFII mRNA expression were found in all cell lines in the NCI60 panel of human cancer cell lines [180].

\section{Regulation by other transcription factors}

COUP-TFII and Ets-1 have overlapping expression patterns in mesenchymal cells of the mouse gut, spleen, lungs, and other tissues [181]. Members of the ETS family (Ets-1, Ets-2, ETV, PEA3, Spi-1, and ERM) increased murine COUP-TFII-promoter activity in HeLa cells. Steroid receptor coactivators SRC-1/NCOA1, TIF2/SRC2/NCOA2, and RAC3/SRC-3/NCOA3 enhanced the activation of the COUP-TFII promoter [181]. In agreement with this data, SRC-3 and RAR $\alpha$ increased COUP-TFIIpromoter activity in HepG2 human hepatocellular carcinoma cells with atRA treatment. Reciprocally, siRNA knockdown of SRC-3 repressed COUP-TFII expression [182]. We observed that the protein expression (by immunohistochemical staining) of AIB1/SRC3/NCOA3, PEA3, and SRC-1/NCOA1 were correlated with COUP-TFII in breast cancer patient samples [115].

Regulation by altered kinase activity and other signaling pathways

Several factors were reported to alter COUP-TFII expression in pathogenic states. More et al reported that expression of COUP-TFII, but not COUP-TFI, is stimulated by activation of the MAP kinase (MAPK) pathway. Breast cancer cell lines with increased 
MAPK activity, i.e., SKBR3, had a concomitant increase in COUP-TFII expression [183]. In contrast to the idea that MAPK activation increases COUP-TFII expression, MAPK has also been shown to phosphorylate and inactivate PP2A (protein phosphatase 2A), leading to a suppression of COUP-TFII expression in human peripheral blood CD34+ cells [184]. Inactivation of PP2A also inhibits sonic hedgehog-induced COUPTFII expression in P19 cells [185]. PP2A is inhibited by the FOXO transcription factors, including FOXO1 [186]. COUP-TFII expression is induced by FOXO1 in pancreatic beta cells and hepatocytes [171], highlighting the highly cell type-specific nature of these pathways. MAPK activity may lead to increased COUP-TFII expression in certain conditions, while it may alternatively repress COUP-TFII in others. Taken together, these data suggest a possible feedback loop in certain cell types (Figure 4).

In addition to MAPK activation, Notch signaling is also dysregulated in many types of cancer. Increased Notch signaling has been implicated in carcinogenesis and metastasis and is also involved in regulation of endothelial cell proliferation and angiogenesis [187,188]. In breast cancer, Notch and its ligand Jagged1 upregulate the expression of Slug, a transcriptional repressor of E-cadherin important in metastatic progression [189]. Notch signaling has also been implicated in the amplification of HER2 and survival of tumor initiating cells [190] and cancer stem cells [188,191,192]. Activation of the Notch pathway confers cancer-like properties and apoptosis-resistance to normal breast epithelial cells [193]. Regulation of COUP-TFII by Notch signaling has been reported in endothelial cells of both arterial and venous origin and in mouse studies $[163,194,195]$. Notch can suppress COUP-TFII and Prospero-related homeobox domain 1 (Prox1), leading to an arterial rather than lymphatic phenotype in endothelial cells 
[163,194,196]. COUP-TFII, in turn, can also suppress Notch signaling to result in vein rather than artery formation [195]. Transforming growth factor- $\beta 1$ (TGF $\beta 1$ ) suppresses COUP-TFII expression in keratinocytes and fibroblasts leading to induction of collagen type VII (COL7A1) expression [197] and in vascular progenitor cells to negatively regulate lymphvasculogenesis [198]. Whether COUP-TFII is regulated via Notch and TGF $\beta 1$ signaling has not yet been explored in cancer.

Amplification of $\mathrm{Wnt} / \beta$-catenin signaling has been widely reported in cancer [199]. In normal tissues, $\beta$-catenin signaling is controlled through signals leading to its phosphorylation by a multiprotein destruction complex and subsequent degradation. In breast and other cancers, increased expression of Wnt ligands leads to maintenance of $\beta$ catenin activation by preventing its degradation [199]. $\beta$-catenin signaling has many outcomes, such as normal mammary morphogenesis and ductal maturation; however, sustained activation, through a variety of mechanisms, leads to carcinogenesis [199]. ChIP assays demonstrated that $\beta$-catenin/TCF7L2 (T-cell factor 7-like 2 or transcription factor 7-like 2) bind the promoter of COUP-TFII to activate expression, resulting in suppression of adipocyte differentiation [128]. COUP-TFII is expressed in mouse liver and pancreatic $\beta$-cells and plays roles in the maintenance of glucose homeostasis and insulin sensitivity $[171,200]$. Boutant et al also reported that $\beta$-catenin/TCF7L2 induces COUP-TFII expression in the pancreas, and that COUP-TFII expression was necessary for normal $\beta$-cell function and glucose tolerance in mice [201]. The influence of $\beta$ catenin signaling on COUP-TFII expression in cancer has yet to be examined. 


\section{ROLE OF COUP-TFII IN CANCER}

\section{Angiogenesis}

Many studies of COUP-TFII involve its regulation of the angiogenesis pathway. Under normal conditions, angiogenesis is not active after the time of vasculature development during embryogenesis. However, upon progression of a tumor's growth, activation of angiogenesis leads to the formation of new blood vessels to support the tumor [202]. COUP-TFII is necessary during normal development for angiogenesis and lymphangiogenesis, as evidenced by the impaired vessel formation and embryonic lethality in COUP-TFII knockout mice [111,203]. The expression of many proangiogenic factors is modulated by COUP-TFII, including members of the vascular endothelial growth factor (VEGF) family and their receptors. VEGF induces angiogenesis and lymphangiogenesis by activating tyrosine kinase receptors and upregulates endothelial cell proliferation and migration [204]. In a model of pancreatic islet tumorigenesis, ablation of COUP-TFII increased VEGFR-1 expression, impairing VEGFR-2 signaling and reducing angiogenesis [205]. Metastasis to regional lymph nodes was reduced as a result, implying that COUP-TFII may have a pro-angiogenic, pro-metastatic role in pancreatic cancer [205]. Similarly, ablation of COUP-TFII decreased tumorigenesis in B16-F10 melanoma and Lewis lung carcinoma mouse xenografts, and reduced tumorigenesis and metastasis in a spontaneous mouse mammary tumor model. These effects were attributed to a decrease in blood vessel density in COUP-TFII-deficient mice [206].

In addition to regulating VEGFR expression, COUP-TFII can also affect angiogenesis via regulation of angiopoetin-1 (Ang-1), through binding to an Sp1 site in 
the promoter region. The induction of Ang-1 is partially responsible for the effects of COUP-TFII, as overexpression of Ang-1 allowed for recovery of angiogenesis in COUPTFII-deficient mice [206].

Lymphangiogenesis can also contribute to metastasis by allowing the spread of tumor cells to lymph nodes [207,208]. COUP-TFII regulates tumor lymphangiogenesis via inducing expression of VEGF-C and neuropilin-2, a coreceptor for VEGF-C $[203,209]$. In a murine model of pancreatic islet tumorigenesis, COUP-TFII deletion resulted in impaired lymphangiogenesis and reduced metastasis [205]. Concordant with a role for COUP-TFII in lymphangiogenesis, Kang et al reported that Notch suppresses COUP-TFII expression, along with Prox1, in human primary dermal lymphatic endothelial cells to signal for arterial rather than lymphatic differentiation [163]. Suppression of COUP-TFII resulted in an increase in VEGF signaling by activating expression of VEGFR-2, a VEGF receptor whose signaling can feedback to increase activation of Notch signaling [163].

COUP-TFII induction by 9cRA was also shown to promote network formation but not cell fusion in SKBR3 breast cancer cells, suggesting a role in the endothelial transdifferentiation pathway as a necessary part of vascular formation [210]. Taken together, these data indicate that COUP-TFII may regulate angiogenesis and lymphangiogenesis, primarily through modulation of VEGF and its receptor in a cell context-dependent manner.

\section{Invasion and metastasis}


In addition to stimulation of angiogenesis, COUP-TFII may have other distinct roles in regulation of tumor growth and metastasis. Transfection with COUP-TFII in A549, H520, and H441 lung cancer cells and MDA-MB-231 breast cancer cells was reported to increase migration and invasion [211]. Navab et al found that COUP-TFII upregulated the expression of extracellular matrix-degrading proteinases matrix metalloproteinase 2 (MMP2) and urokinase-type plasminogen activator (uPA) [211]. MMP2 and uPA are known to play critical roles in cancer, particularly in angiogenesis and metastasis [212]. High levels of uPA are predictive of recurrence but also of a favorable response to adjuvant chemotherapy in breast cancer patients [213]. Interestingly, it has also been reported that uPA expression is dependent on Notch signaling in MDA-MB-231, MDA-MB-468, and HCC1143 breast cancer cells [214]. COUP-TFII and MMP2 expression were also positively correlated in a breast tumor microarray [115], further indicating a potential relationship between COUP-TFII and extracellular matrix degradation. In contrast, COUP-TFII decreased cell motility when transfected into LY2 tamoxifen-resistant breast cancer cells, while having no significant effect on invasion [116].

\section{Estrogen receptor and clinical outcome}

Nagasaki et al demonstrated that COUP-TFII expression was correlated with ER $\alpha$ status and indices of poor clinical outcome (clinical stage, lymph node status, histological grade) in human breast tumor samples, indicating COUP-TFII may play a role in cancer progression [209]. We also found that COUP-TFII and ER $\alpha$ expression were correlated in a human breast tissue/tumor microarray, but instead noted an inverse relationship 
between COUP-TFII expression and TNM (tumor, node, metastasis) classification [115]. Similar findings were observed at the mRNA level by examining breast tumor mRNA transcriptomes in Oncomine [115]. COUP-TFII expression was significantly higher in $\mathrm{ER} \alpha+$ breast cancer samples and significantly lower in metastatic samples [115]. These findings indicate a function for COUP-TFII in inhibiting tumor progression. A positive correlation with $\mathrm{ER} \alpha$ is consistent with a previous report that siRNA knockdown of ER $\alpha$ in MCF-7 breast cancer cells decreased COUP-TFII expression and treatment with estradiol increased the expression of COUP-TFII [116]. ER $\alpha$ is a positive prognostic factor in breast tumors and is the target of endocrine-targeted cancer therapeutics such as the selective estrogen receptor modulators (SERMs) tamoxifen and raloxifene [48]. COUP-TFII, but not COUP-TFI, is reduced in tamoxifen-resistant human breast cancer cells, and re-expression of COUP-TFII can restore tamoxifen-sensitivity [116]. As ER $\alpha$ expression is important in keeping breast cancer cells responsive to treatment, the correlation of COUP-TFII and ER $\alpha$ further demonstrates a beneficial role for COUPTFII, highlighting its potential importance in maintaining differentiation and endocrine sensitivity.

In contrast to a role for COUP-TFII in maintaining antiestrogen sensitivity, Holbeck et al reported that cancer cells in the NCI60 panel expressing low levels of COUP-TFII showed higher sensitivity to microtubule-targeting drugs vinblastine, colchicines, and taxol [180]. These data demonstrate that both cell type-specific as well as drug-specific mechanisms may determine the role of COUP-TFII in influencing treatment response. 


\section{Steroidogenesis}

COUP-TFII expression was reported to be high in aldosteroma, with an inverse correlation to adrenal steroidogenesis [215]. These data also indicated an inverse correlation between COUP-TFII expression and CYP17A1 expression, with COUP-TFII inhibiting CYP17A1 in aldosteroma [215]. COUP-TFII competed with SF-1 for binding to overlapping sites within the promoters of the CYP17A1 [216,217], CYP11A1, and STARD1 genes in rat Leydig cells and to suppress testosterone production [216]. COUPTFI and COUP-TFII both repressed angiotensin II-stimulated STARD1 (StAR) in bovine adrenal glomerulosa cells in primary culture [218]. COUP-TFII also competed with SF-1 for the human aromatase P450 promoter II in primary endometriotic stromal cells and suppressed aromatase expression.[219]. Overexpression of SF-1 in primary endometriotic stromal cells outcompeted the normal protective effect of COUP-TF (whether COUP-TFI or COUP-TFII was involved was unclear since both were equally expressed at the mRNA level) resulting in high local aromatase expression in endometriosis [219]. COUP-TFII was reported to bind the S1 silencer region of the human aromatase gene and suppress transcription in MCF-7 cells [220]. Indeed, the decreases in COUP-TFI, EAR $\gamma$, EAR-2, Snail and Slug in breast cancer were suggested to increase aromatase expression [221]. Thus, the downregulation of COUP-TFII expression that we observed in endocrine-resistant breast cancer cells [116] would be expected to increase aromatase and thus increase local estrogen production. However, whether increased COUP-TFII suppresses local androgen or estrogen biosynthesis in breast tissue is unknown. Local conversion of adrenal androgens to estrogens by aromatase is the target of AI therapy for post-menopausal women. However, there are 
androgen metabolites, e.g., $3 \beta$-adiol, that bypass aromatase which activate $\mathrm{ER} \alpha$ and $\mathrm{ER} \beta$ and may play a role in AI resistance [222,223]. Overall, the literature supports a negative role for COUP-TFII in regulating steroid hormone synthesis and further studies addressing COUP-TFII regulation of aromatase gene expression in local estrogen production in breast [224] and lung [225] adenocarcinomas would be of merit.

\section{CONCLUSIONS}

The studies reviewed here indicate that COUP-TFII is regulated and is functionally active to regulate target gene transcription in a cell type-dependent manner. There is evidence that COUP-TFII may perform both pro- and anti-tumorigenic roles. COUP-TFII has been reported to increase angiogenesis and lymphangiogenesis, both increase and decrease tumor metastasis, lead to favorable and unfavorable therapeutic outcome in cancer therapy, and suppress steroidogenesis. Qin et al reported that COUPTFII was not expressed in tumor cells, but rather was instead found in high concentration in the surrounding blood vessels that support tumor growth and spread [206]. This indicates a crucial point of consideration about the nature of COUP-TFII in cancer formation and progression: the function of COUP-TFII within cancer cells versus in the surrounding tumor microenvironment and other cell types. Tissue type is clearly an important determinant in deciphering the oncogenic or tumor-suppressive nature of COUP-TFII. Many studies published to date involve the regulation and role of COUPTFII during development and in non-cancerous disease states. The full applicability of these studies to our knowledge of the role of COUP-TFII in carcinogenesis and cancer 
progression remains to be seen. Future studies are necessary to elucidate the complex nature of this vital nuclear receptor.

\section{DISSERTATION SPECIFIC AIMS AND HYPOTHESES}

Despite the initial efficacy of tamoxifen and other antiestrogens, approximately $40 \%$ of patients relapse and die from metastatic disease, in part because the cancer cells become endocrine-resistant [56]. Identification of proteins and pathways key in the progression to endocrine resistance is essential for the development of improved treatments for patients. Our lab has previously reported that the expression of COUPTFII, but not COUP-TFI, is reduced upon progression to endocrine resistance, and that overexpression of COUP-TFII can restore endocrine sensitivity [116]. As reviewed in chapter 2, many reports on the cellular roles of COUP-TFII are contradictory and fail to provide insight necessary to understand the mechanism of COUP-TFII action in endocrine-resistant breast cancer [226]. The overall goal of this study was to identify and evaluate the function of proteins that interact with COUP-TFII to define the role of COUP-TFII in breast cancer and endocrine sensitivity. COUP-TFII may be both a useful biomarker to predict tamoxifen-sensitivity as well as a target to restore endocrine sensitivity to resistant cells. The overall hypothesis was that the activity of COUP-TFII in maintenance of endocrine sensitivity and cell differentiation is dependent on its interacting proteins. 


\section{SPECIFIC AIM 1: Characterize the interaction of COUP-TFII and nucleolin.}

I tested the hypothesis that COUP-TFII and nucleolin interact directly in MCF-7 and T47D breast cancer cells. COUP-TFII and nucleolin interaction was evaluated in MCF-7 and T47D cells through co-immunoprecipitation. In vitro binding was assessed through a maltose binding protein (MBP) pulldown assay. COUP-TFII and nucleolin expression were analyzed in human breast cancer patient samples through immunohistochemistry.

SPECIFIC AIM 2: Determine how the interaction of COUP-TFII and nucleolin affects target gene expression in breast cancer cells.

I tested the hypothesis that nucleolin functions as a coregulator for COUP-TFII in the expression of the COUP-TFII target gene RARB2. The ability of nucleolin to function as a coactivator for COUP-TFII was analyzed in MCF-7 and T47D cells. Transient transfection, siRNA knockdown, luciferase assays, and other assays were used to determine the contribution of nucleolin to RARB2 expression.

\section{SPECIFIC AIM 3: Determine if COUP-TFII expression modulates NFkB activity in} endocrine-resistant breast cancer cells.

I tested the hypothesis that COUP-TFII suppresses NFKB activity in breast cancer cells through a mechanism involving suppression of NFאB transcription. The effect of COUPTFII on NFKB activity was assayed in endocrine-sensitive MCF-7 versus endocrineresistant LCC9 breast cancer cells using luciferase assays and electrophoretic mobility shift assays (EMSA). NFkB subunit and target gene expression was examined using QRT-PCR and western blots. 
SPECIFIC AIM 4: Determine the identity of proteins that interact with COUP-TFII in tamoxifen-treated MCF-7 human breast cancer cells.

I tested the hypothesis that 4-OHT treatment changes the interaction of COUP-TFII with other nuclear proteins in MCF-7 endocrine-sensitive cells and that these proteins are important in mediating the ability of antiestrogens to inhibit cell proliferation. MCF-7 breast cancer cells were transiently transfected with FLAG-tagged COUP-TFII and treated with ethanol (EtOH, vehicle control) or 4-OHT before immunoprecipitation was performed using a FLAG antibody to capture COUP-TFII and associated proteins. Mass spectrometry identified proteins interacting with COUP-TFII specifically when treated with EtOH versus 4-OHT. Further experiments including co-immunoprecipitation, transient transfection, western blots, and QRT-PCR were performed to analyze the role of the identified COUP-TFII-interacting proteins. 


\section{CHAPTER III - IDENTIFICATION AND CHARACTERIZATION OF NUCLEOLIN AS A COUP-TFII COACTIVATOR OF RETINOIC ACID RECEPTOR $\beta$ TRANSCRIPTION IN BREAST CANCER CELLS}

The text of this chapter was published in PLOS ONE 7(5): e38278, 2012. doi:10.1371/journal.pone.0038278

\section{INTRODUCTION}

COUP-TFI $(N R 2 F 1)$ and COUP-TFII $(N R 2 F 2)$ are 'orphan' members of the steroid/nuclear receptor (NR) superfamily [107]. COUP-TFs regulate gene transcription in a cell- and gene- specific manner. COUP-TFII differs from COUP-TFI at the Nterminus, but is conserved within the DNA binding and ligand binding domains (DBD and LBD) [107]. Gene knockout mice demonstrated that COUP-TFI (Nr2f1) and COUPTFII (Nr2f2) have distinct roles during embryogenesis, notably in the nervous and cardiovascular systems, respectively [111,227]. Although COUP-TFs are classified as orphan receptors, as they have no currently established physiological ligands, the crystal structure of the COUP-TFII LBD showed that its "auto-repressed conformation" was relieved by 9-cis and all-trans retinoic acids (9cRA and atRA) that bind the LBD with 17-26 $\mu \mathrm{M}$ affinity [120]. 
While the precise gene changes and epigenetic events that lead to breast tumorigenesis are still under investigation [228-230], estrogens are well-established risk factors in breast cancer [231]. Adjuvant endocrine therapies including the use of antiestrogens, e.g., tamoxifen (TAM), and aromatase inhibitors (AI), e.g., letrozole, are effective in reducing disease recurrence in many patients [102]. Antiestrogens, including TAM and fulvestrant, work by targeting estrogen receptor $\alpha(E R \alpha)$ because of its proliferative activity in breast tumors [232,233]. AI work by blocking the synthesis of estrogens from androgenic precursors including androstenedione and testosterone [234].

Altered gene expression can dictate both the formation of tumors and patient response to treatment. In breast cancer, conflicting evidence has been reported on the nature of COUP-TFII in either promoting or inhibiting cancer formation, as well as influencing patient survival with adjuvant therapy. COUP-TFII is not expressed in basallike, triple negative, dedifferentiated MDA-MB-231 and is lower in tamoxifen (TAM)/endocrine-resistant LCC9 and LY2 breast cancer cells than in parental endocrinesensitive MCF-7 cells, whereas COUP-TFI expression is similar [116], suggesting a role in maintenance of differentiation and endocrine sensitivity. In agreement with this data, COUP-TFII was reduced in some ER $\alpha$-null breast cancer cell lines [167]. These results suggest that, like ER $\alpha$, loss of COUP-TFII may be considered an indicator of poor prognosis. Other reports suggested that COUP-TFII may play a role in mammary tumor formation in mice and that COUP-TFII expression in human breast tumors is associated with reduced survival $[206,209]$. These conflicting findings may be resolved through further investigation of the activities of COUP-TFII in breast cancer. 
The function of COUP-TFs as transcription factors that can either suppress or stimulate gene transcription is dependent on interactions with other proteins. COUP-TFI [127] and COUP-TFII [119] interact with corepressors NCoR and SMRT. Proteins interacting with COUP-TFI include Sp1 [235]; the viral transactivator Tat [236]; CTIP1 and CTIP2, HDACs 1 and 2, and a nucleosome remodeling and deacetylation (NuRD) complex [237]; ER $\alpha$ [130,238]; AhR [239]; and many coregulators (reviewed in [156]). Twenty-four proteins interacted with HA-FLAG-COUP-TFI in stably-transfected HeLaS3 cells [114]. Interaction of ORCA with the COUP-TFII LBD stimulated transcriptional activation of the rat hydratase-dehydrogenase gene promoter in transiently transfected Bsc40 monkey kidney cells [123]. COUP-TFII interacted with the hinge domain of the glucocorticoid receptor $\alpha(\mathrm{GR} \alpha)$ and repressed phosphoenolpyruvate carboxykinase gene transcription [124]. No one has, to our knowledge, reported proteomic identification of COUP-TFII-interacting proteins.

The focus of the present study was to identify proteins that interact with COUPTFII in MCF-7 cells to gain new insights into COUP-TFII's role in breast cancer. Nucleolin was identified among the nuclear proteins interacting with COUP-TFII. COUP-TFII-nucleolin interaction was confirmed by co-immunoprecipitation. This study reports a significant inverse association of COUP-TFII with breast tumor grade. Expression of the tumor suppressor retinoic acid receptor $\beta 2$ (RAR $\beta 2)$, reduced in breast cancer [240,241], and dependent on COUP-TFII [118] was increased by nucleolin overexpression. Our data indicate that nucleolin plays a coregulatory role in COUP-TFII transcriptional regulation of $R A R B 2$. 


\title{
MATERIALS AND METHODS
}

\author{
Chemicals
}

4-hydroxytamoxifen (4-OHT) and 9-cis and all-trans retinoic acid (9cRA and atRA) were from Sigma (St. Louis, MO). ICI 182,780 (Fulvestrant) was from Tocris (Ellisville, MO). Sequences of AS1411 (AGRO100, an antiproliferative, 26-mer G-rich oligonucleotide) and an inactive negative control C-rich control oligonucleotide (CRO) were reported [242] and purchased from Integrated DNA Technologies, Inc. (Coralville, IA).

\section{Antibodies and reagents}

The following antibodies were purchased: polyclonal COUP-TFII (Abcam, Cambridge, MA); monoclonal (mAB) anti-human COUP-TFII (R\&D systems, Minneapolis, MN; PP-H7147-00. 2ZH7147H); mAB anti-FLAG M2 and $\beta$-actin (Sigma); polyclonal nucleolin (NB600-241, Novus Biologicals, Littleton, CO), monoclonal nucleolin/C23 (MS-3; Santa Cruz Biotechnology, Santa Cruz, CA); MBPprobe (R3.2; Santa Cruz Biotechnology); and HDAC-1 (Santa Cruz Biotechnology). HRP-conjugated secondary antibodies were from GE Healthcare (Piscataway, NJ).

Goat anti-rabbit and anti-mouse magnetic beads were from Thermo Scientific (Waltham, MA). In vitro transcription/translation used PROTEINScript II kit (Ambion, Austin, TX) or TNT Quick Coupled Transcription/Translation (Promega, Madison, WI).

Plasmid construction 
Human COUP-TFII cDNA was amplified from DNA from MCF-7 cells using Pfx DNA polymerase (Invitrogen, Carlsbad, CA). The forward primer contained an EcoRI restriction site (5'-CCGAATTCGATATGGCAATGGTAGTTAGCACG-3') and the reverse primer was designed to remove the stop codon from COUP-TFII and add a XhoI restriction site (5'-GTCCTCGAGTCGTTGAATTGCCATATACGGCCA-3'). The resulting fragment was cloned into pIRES-GFP-1a (Stratagene, Santa Clara, CA) to construct a C-terminal FLAG-tagged COUP-TFII expression plasmid (pIRES-COUPTFII-FLAG). The inclusion of COUP-TFII-FLAG in the resulting pIRES-COUP-TFIIFLAG plasmid was verified by DNA sequencing and western blot analysis (Appendix I, Figure S1).

\section{Cell culture}

MCF-7 and T47D breast cancer cells were purchased from ATCC and used at passage $<10$. T47D were grown in RPMI 1640 (Invitrogen) supplemented with 5\% FBS and $6 \mu \mathrm{g} / \mathrm{ml}$ insulin (Sigma). MCF-7 cells were maintained as described [116].

\section{Affinity purification and identification of COUP-TFII-FLAG interacting proteins}

One mg of whole cell extract (WCE), prepared as described in [116], from MCF7 cells transfected $(24 \mathrm{~h}$ ) with pIRES-COUP-TFII-FLAG as described in Methods S1 (Appendix I) was incubated with EZ view ${ }^{\mathrm{TM}}$ Red ANTI-FLAG® M2 Agarose Affinity gel (Sigma) overnight $(\sim 16 \mathrm{~h})$ at $4^{\circ} \mathrm{C}$ with constant rotation. COUP-TFII-FLAG interacting proteins were eluted using two methods: 1) $0.1 \mathrm{M}$ glycine, $\mathrm{pH} \mathrm{3.5,15}$ min at 
room temperature with constant rotation; 2) an additional incubation with $0.1 \mathrm{M}$ glycine, $\mathrm{pH} 3.5,5 \mathrm{~min}$ at $95^{\circ} \mathrm{C}$ (Appendix I, Fig. S2A).

Protein identification by multidimensional protein identification technology (MudPIT)

Proteins eluted from the FLAG-affinity gel were trypsin digested and processed for mass spectrometry as detailed in Methods S1 (Appendix I). MS/MS spectra of the peptides were acquired by Q-TOF mass spectrometer (Waters, Milford, MA) in data dependent mode. Proteins were identified by comparing MS/MS spectra with sequences in Swiss-Prot database by ProteinLynx from Waters.

\section{Co-immunoprecipitation (co-IP) and immunoblotting}

Nuclear and cytosolic proteins were harvested in lysis buffer (10mM HEPES pH 7.9, $1.5 \mathrm{mM} \mathrm{MgCl}_{2}$, and $10 \mathrm{mM} \mathrm{KCl}$ ) containing $0.1 \mathrm{M} \mathrm{DTT}$, protease and phosphate inhibitors (Roche, Indianapolis, IN). Following centrifugation, the supernatant containing cytosolic extract $(\mathrm{CE})$ was collected. The pellet was resuspended in nuclear extraction buffer (20 mM HEPES pH 7.9, $1.5 \mathrm{mM} \mathrm{MgCl}_{2}, 0.4 \mathrm{M} \mathrm{NaCl}, 0.2 \mathrm{M}$ EDTA, and $25 \%$ (v/v) glycerol), $0.1 \mathrm{M}$ DTT, protease and phosphatase inhibitors. Nuclei were disrupted by sonication and the nuclear extracts (NE) were collected after centrifugation.

For IP, $4 \mu \mathrm{g}$ COUP-TFII polyclonal antibody, nucleolin monoclonal antibody, rabbit or mouse IgG (Abcam, Santa Cruz) were added to $250 \mu \mathrm{l}$ prewashed MagnaBind goat anti-rabbit or anti-mouse IgG beads in RIPA buffer (Sigma) containing DTT, protease and phosphatase inhibitors for $30 \mathrm{~min}$ at $4^{\circ} \mathrm{C}$. 200-400 $\mu \mathrm{g}$ NE was added and incubated for $4 \mathrm{~h}$ at $4^{\circ} \mathrm{C}$. Antibody-bound beads were incubated with buffer without NE 
as an additional negative control. Beads were washed $2 \mathrm{x}$ with RIPA buffer, resuspended in 1x Laemmli loading buffer (BioRad, Hercules, CA), separated by SDS-PAGE and analyzed by western blot [116].

In vitro transcribed-translated COUP-TFII interaction with purified recombinant maltose binding protein (MBP)-nucleolin fusion proteins

Extracts from E. coli expressing MBP-tagged nucleolin constructs, a gift from Dr. Nancy Maizels [243], were prepared in column buffer (CB, $20 \mathrm{mM}$ Tris- $\mathrm{HCl}$ (pH 7.4), $0.2 \mathrm{mM} \mathrm{NaCl}, 1 \mathrm{mM}$ EDTA) with $0.1 \mathrm{mM}$ PMSF. $200 \mu \mathrm{g}$ crude extract was incubated with $100 \mu \mathrm{L}$ amylose resin (New England Biolabs, Ipswich, MA) for $2 \mathrm{~h}$ at $4^{\circ} \mathrm{C}$. After washing with $\mathrm{CB}, 20 \mu \mathrm{L}$ of in vitro transcribed/translated COUP-TFII (pIRES-COUPTFII-FLAG) was added to the amylose resin for $2 \mathrm{~h}$ at $4^{\circ} \mathrm{C}$. After washing $3 \mathrm{x}$ with CB, bound proteins were eluted with $50 \mu \mathrm{L}$ of $1 \mathrm{x}$ Laemmli loading buffer.

\section{Immunofluorescence staining of COUP-TFII and nucleolin}

MCF-7 cells were grown on culture slides (BD Biosciences, Bedford, MA) and fixed with cold methanol. Cells were permeabilized with $0.2 \%$ Triton X-100. After blocking with $10 \%$ BSA in PBS for $1 \mathrm{~h}$, primary monoclonal COUP-TFII (R\&D) and polyclonal nucleolin (Novus Biologicals) antibodies were added (1:100) for $2 \mathrm{~h}$. The cells were stained with secondary anti-mouse antibody labeled with DyLight ${ }^{\mathrm{TM}} 488$ or anti-rabbit antibody labeled with rhodamine (TRITC) (Jackson ImmunoResearch, West Grove, PA) (1:500). Cells were incubated with Hoechst (2,5'-Bi-1H-benzimidazole, 
Invitrogen) for $10 \mathrm{~min}$. Images were captured using an Olympus FV1000 confocal microscope with a 40x objective lens using FluoView ${ }^{\mathrm{TM}}$ software.

Immunohistochemistry of breast tissue microarrays (TMA)

COUP-TFII and nucleolin immunohistochemistry (IHC) was performed using commercial breast tissue microarrays BR961 and BR963 (U.S. Biomax) or an in-house TMA constructed following ethical approval from St. Vincent's University Hospital Ethics Board with tissue from 332 primary breast patients, following written informed consent, as previously described $[61,244]$. Data on the patients included pathological characteristics (tumor size, grade, lymph node status, estrogen receptor status) as well as treatment with radiotherapy, chemotherapy or tamoxifen. Follow-up data, median 7.72 years, was collected on the patients to determine disease free and overall survival. Staining was called by two independent observers using the Allred scoring system [61]. Xenografted MCF7 and HCT116 tumors were used as positive and negative controls, respectively (data not shown). Anti-Nucleolin antibody (Clone 4E2, Abcam) was diluted at 1:500 with overnight incubation at $4^{\circ} \mathrm{C}$ for BR961 and BR963. A metastatic melanoma was used as a positive control for nucleolin (data not shown). COUP-TFII and nucleolin staining were expressed as $\mathrm{H}$-score: product of intensity ( 0 to 3 scale, $0=$ no expression, 3 strongest expression) and frequency (fraction positive, $0-100 \%$ ).

\section{Statistical Evaluation of IHC}

The univariate associations between COUP-TFII and nucleolin $\mathrm{H}$-scores and categorical predictors used the Kruskal-Wallis test [61]. A multiple linear regression 
model was used to fit with COUP-TFII and nucleolin H-scores against pathology, tumor grade, and TNM, classification. The TNM staging system classifies tumors according to disease progression based on the tumor size $(\mathrm{T})$, regional lymph node involvement $(\mathrm{N})$, and distant metastasis (M). Upon assignment of TNM, tumors can further be designated into a condensed grade/stage (I-IV) based on disease severity [245]. Comparisons in the ER $\alpha$-positive invasive ductal carcinoma subset and among TNM classification within tumor grades were examined (t-test). Fisher's exact test was used for categorical variables to compare two proportions. Kaplan Meier estimates of survival functions were computed and the Wilcoxon test was used to compare survival curves. Two-sided $\mathrm{P}$ values of $<0.05$ were considered to be statistically significant.

\section{Transient transfection}

MCF-7 and T47D cells were transfected with a constant amount of total plasmid DNA, pcDNA 3.1 (Promega), pCMV-Tag2 (Stratagene), pcDNA 3.1-mCOUP-TFII (kindly provided by Drs. Sophia and Ming-Jer Tsai [246]), pCMV2-nucleolin [247] using Fugene 6 or HD (Roche) for $24 \mathrm{~h}$ prior to treatment with $10 \mu \mathrm{M} \mathrm{CRO}$ (negative control), AS1411, or random oligomer (RO, 5'-GTTCAGCAGTCACGATTCAGTCCAGT-3') for 6 or $24 \mathrm{~h}$, as indicated. Where indicated, cells were co-treated with $1 \mu \mathrm{M}$ atRA or 9cRA for $6 \mathrm{~h}$. Transient transfection of MCF-7 cells with the RARB promoter tk-luciferase reporter (kindly provided by Dr. Richard M. Niles [248]) and pTK-Renilla (Promega), for dual luciferase reporter assays, as described [130].

RNA Isolation, RT-PCR and Quantitative Real-Time-PCR (QRT-PCR) 
RNA was extracted from cells using Trizol (Invitrogen) or RNeasy (Qiagen, Valencia, CA). The High Capacity cDNA Reverse Transcription kit (Applied Biosystems) was used to reverse transcribe total RNA. QRT-PCR for RARB2, NCL, ESR1, ESR2, GAPDH, and 18S, using Taqman primers and probes as Assays-onDemand, was performed in the ABI PRISM 7900 SDS 2.1 (Applied Biosystems, Carlsbad, CA). COUP-TFII (NR2F2) and RRIG1 [249] mRNA expression was measured by QRT-PCR using the SYBR green method and normalized by GAPDH [116]. Analysis and fold differences were determined using the comparative CT method. Fold change was calculated from the $\Delta \Delta \mathrm{CT}$ values with the formula $2^{-\Delta \Delta \mathrm{CT}}$ and data are relative to EtOH-treated and control vector transfected cells.

siRNA transfection

For nucleolin, cells were transfected for $48 \mathrm{~h}$ with $25 \mathrm{nM}$ (MCF-7) or $10 \mathrm{nM}$ (T47D) nucleolin Stealth Select RNAi or Stealth RNAi Negative Control (Invitrogen) using Lipofectamine RNAiMAX (Invitrogen). For COUP-TFII, MCF-7 cells were transfected for $48 \mathrm{~h}$ with $100 \mathrm{pmol}$ NR2F2 Silencer Select siRNA (Ambion). Following transfection, cells were treated for $6 \mathrm{~h}$ with $1 \mu \mathrm{M}$ atRA.

Chromatin immunoprecipitation (ChIP)

MCF-7 cells were transfected with pIRES-COUP-TFII-FLAG or empty vector for $24 \mathrm{~h}$ and serum starved for $48 \mathrm{~h}$ in medium containing 5\% dextran-coated charcoal stripped FBS (DCC-FBS) (Atlanta Biologicals, Lawrenceville, GA). Cells were treated with $1 \mu \mathrm{M}$ atRA or EtOH for $1 \mathrm{~h}$ before crosslinking with $1 \%$ formaldehyde for $5 \mathrm{~min}$. 
ChIP was performed using MAGnify ChIP (Invitrogen). Lysates were incubated with anti-FLAG M2 antibody (Sigma) or mouse IgG (Invitrogen). The following primers were used for PCR to amplify the region of the RARB2 promoter containing a COUPTFII binding site [118]: F 5'-CAGGGCTGCTGGGAGTTTTTA-3' and R 5'GGCATCCCAGTCCTCAAACAGC-3'. Quantitation was performed as described in [250].

Statistical analysis

Values are reported as \pm SEM. Student's $t$ test was used for comparisons between control and treatment. One way ANOVA was used for multiple comparisons followed by Student-Newman-Keuls or Dunnett's post-hoc tests using GraphPad Prism. $P$ values considered statistically significant are indicated.

\section{RESULTS}

Identification of COUP-TFII-associated proteins

C-terminal FLAG-tagged COUP-TFII was overexpressed in MCF-7 cells ( 2-fold higher expression compared to COUP-TFII endogenous expression; Appendix I, Fig. S1 and S2) and interacting proteins were captured by immunoprecipitation (IP) with antiFLAG-affinity gel (Appendix I, Fig. S2A). The negative control was MCF-7 cells transfected with the pIRES-GFP-1a parental vector and parallel purification of nonspecific interacting proteins (Appendix I, Table S1). The capture of COUP-TFIIFLAG by the anti-FLAG affinity gel was demonstrated (Appendix I, Fig. S2C). Serial glycine steps eluted COUP-TFII-FLAG-associated proteins (Appendix I, Fig. S3). In the 
first elution, 18 proteins having a 'moderate association' with COUP-TFII including hsp70, an established NR chaperone that interacts with COUP-TFI [114], were identified (Appendix I, Table S2). The second elution identified 36 more 'strongly associated' nuclear proteins, i.e., ribnucleoproteins, histones, DNA repair proteins, and RNA binding proteins, and nucleolin (Table 2), reflecting COUP-TFII nuclear localization.

Nucleolin is a multifunctional protein with roles in processes including transcription, ribosome biogenesis, DNA replication, histone chaperone/chromatin remodeling, apoptosis, and macropinocytosis [251-254]. There are several examples of nucleolin functioning as a transcription factor or as a coregulator through its interactions with other proteins [255-257]. Because nucleolin plays multiple nuclear roles and is a target of anticancer therapy [252], we selected nucleolin for follow-up studies. 
Table 2. Identification of COUP-TFII interacting proteins in MCF-7 cells.

WCE from pCOUP-TFII-FLAG-transfected MCF-7 cells (Fig. 6) was incubated with anti-FLAG affinity gel, eluted with $0.1 \mathrm{M}$ glycine, $\mathrm{pH} 3.5$ for $5 \mathrm{~min}$. at $95^{\circ} \mathrm{C}$, and subjected to MudPIT peptide identification. Matched (No) indicates the number of sequenced peptides that match the full length protein. Coverage indicates the $\%$ of the total protein matched. (Only matches of $>1$ peptide match and/or $>3 \%$ coverage are included.)

\begin{tabular}{|c|c|c|c|c|c|}
\hline Protein name & $\begin{array}{c}\text { Accession } \\
\text { (GI) }\end{array}$ & $\begin{array}{c}\text { Mass } \\
(\mathrm{Mr})\end{array}$ & $\overline{p l}$ & $\begin{array}{l}\text { Matched } \\
\text { (No) }\end{array}$ & $\begin{array}{c}\text { Coverage } \\
(\%)\end{array}$ \\
\hline \multicolumn{6}{|l|}{ Histones } \\
\hline Histone H1.0 & P07305 & 20719 & 11.2 & 1 & 6.7 \\
\hline Histone H2A type 2-C & Q16777 & 5919 & 11.8 & 1 & 16.1 \\
\hline Histone $\mathrm{H} 1.4$ & P10412 & 21721 & 11.4 & 7 & 4.1 \\
\hline \multicolumn{6}{|l|}{$\begin{array}{l}\text { Proteins involved in cell cycle \& } \\
\text { proliferation }\end{array}$} \\
\hline Antigen $\mathrm{KI}-67$ & P46013 & 358525 & 9.8 & 6 & 0.5 \\
\hline $\begin{array}{l}\text { Regulator of chromosome } \\
\text { condensation }\end{array}$ & P18754 & 44941 & 7.7 & 4 & 3.1 \\
\hline $\begin{array}{l}\text { Poly [ADP-ribose] polymerase } 1 \\
\text { (PARP-1) }\end{array}$ & P09874 & 112881 & 9.2 & 7 & 5.7 \\
\hline \multicolumn{6}{|l|}{$\begin{array}{l}\text { Proteins involved in } \\
\text { transcription }\end{array}$} \\
\hline $\begin{array}{l}\text { Activated RNA polymerase II } \\
\text { transcriptional coactivator } \mathrm{p} 15\end{array}$ & P53999 & 14255 & 9.9 & 1 & 8.7 \\
\hline \multicolumn{6}{|l|}{ DNA Repair Proteins } \\
\hline DNA topoisomerase 1 & P11387 & 90669 & 9.6 & 5 & 1.6 \\
\hline $\begin{array}{l}\text { DNA topoisomerase 2-beta } \\
(\text { Topoll } \beta)\end{array}$ & Q02880 & 183152 & 8.4 & 4 & 2.0 \\
\hline $\begin{array}{l}\text { ATP-dependent DNA helicase } 2 \\
\text { subunit } 1\end{array}$ & P12956 & 69668 & 6.6 & 3 & 2.1 \\
\hline $\begin{array}{l}\text { ATP-dependent DNA helicase } 2 \\
\text { subunit } 2\end{array}$ & P13010 & 82521 & 5.7 & 3 & 3.0 \\
\hline $\begin{array}{l}\text { Peptidyl-prolyl cis-trans isomerase } \\
\text { B }\end{array}$ & P23284 & 22728 & 9.6 & 3 & 10.6 \\
\hline \multicolumn{6}{|l|}{ RNA binding proteins } \\
\hline RNA-binding protein FUS & P35637 & 53394 & 9.5 & 3 & 2.7 \\
\hline $\begin{array}{l}\text { Splicing factor, arginine/serine-rich } \\
1\end{array}$ & Q07955 & 27597 & 10.5 & 4 & 12.6 \\
\hline $\begin{array}{l}\text { Splicing factor, arginine/serine-rich } \\
9\end{array}$ & Q13242 & 25526 & 8.9 & 2 & 5.4 \\
\hline $\begin{array}{l}\text { Splicing factor, arginine/serine-rich } \\
7\end{array}$ & Q16629 & 27350 & 11.8 & 2 & 3.8 \\
\hline \multicolumn{6}{|l|}{ Other nuclear proteins } \\
\hline $\begin{array}{l}\text { Nonhistone chromosomal protein } \\
\text { HMG } 14\end{array}$ & P05114 & 10522 & 10.0 & 2 & 24.2 \\
\hline Nucleolin Protein C23 & P19338 & 76167 & 4.6 & 3 & 1.7 \\
\hline Heat shock cognate $71 \mathrm{kDa}$ protein & P11142 & 70854 & 5.4 & 2 & 4.3 \\
\hline GTP-binding nuclear protein Ran & P62826 & 24408 & 7.6 & 2 & 9.7 \\
\hline
\end{tabular}


Heat shock $70 \mathrm{kDa}$ protein 1 (hsp70, HSPA1)
P08107

70009

5.6

2

4.4 
Endogenous COUP-TFII and nucleolin interact in MCF-7 and T47D cells

We next examined endogenous nucleolin-COUP-TFII interaction in untreated MCF-7 cells. IP with a COUP-TFII antibody detected nucleolin interaction in the NE (Fig. 6A), although nucleolin is in CE as well (Appendix I, Fig. S4A). We did not detect COUP-TFII-nucleolin interaction in CE because COUP-TFII is not in the CE (Appendix I, Fig S4A). Reciprocal co-IP of COUP-TFII with nuclear nucleolin was detected (Appendix I, Fig. S4A). Another example of a COUP-TFII- nucleolin co-IP in MCF-7 cells is also provided (Appendix I, Fig. S4C and D). To examine whether COUP-TFII interacts with nucleolin in another luminal breast cancer cell line, we performed IP with a COUP-TFII antibody in T47D cells and confirmed nucleolin-COUP-TFII interaction in the NE (Fig 6B). Immunofluorescence microscopy revealed a pattern of co-localization of endogenous nucleolin and COUP-TFII in the nucleus, but not within the nucleolus nor in the cytoplasm, of MCF-7 cells (Fig. 6C). These data confirm endogenous COUPTFII-nucleolin nuclear interaction. Because the focus of this study is COUP-TFIIinteracting proteins, we did not evaluate COUP-TFI-nucleolin interaction. 
A MCF-7 cells

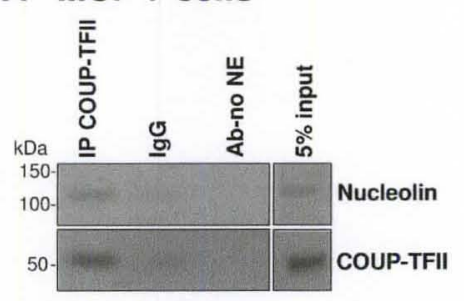

B T47D cells
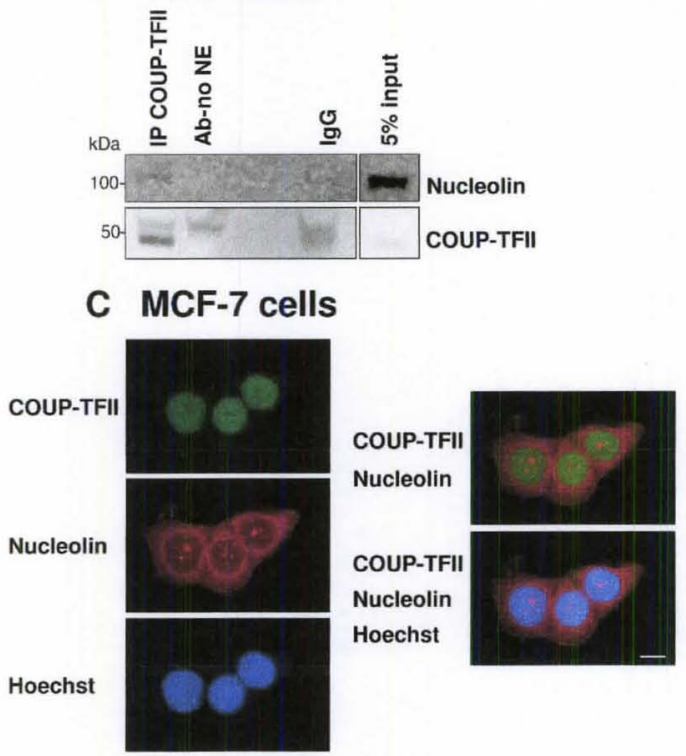

D

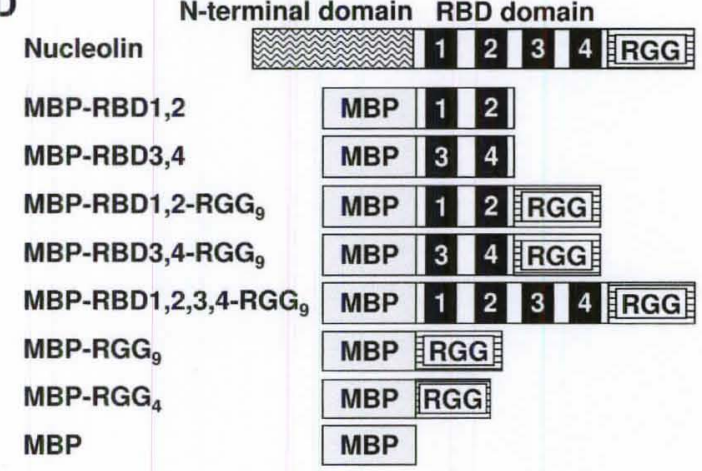

$\mathbf{E}$

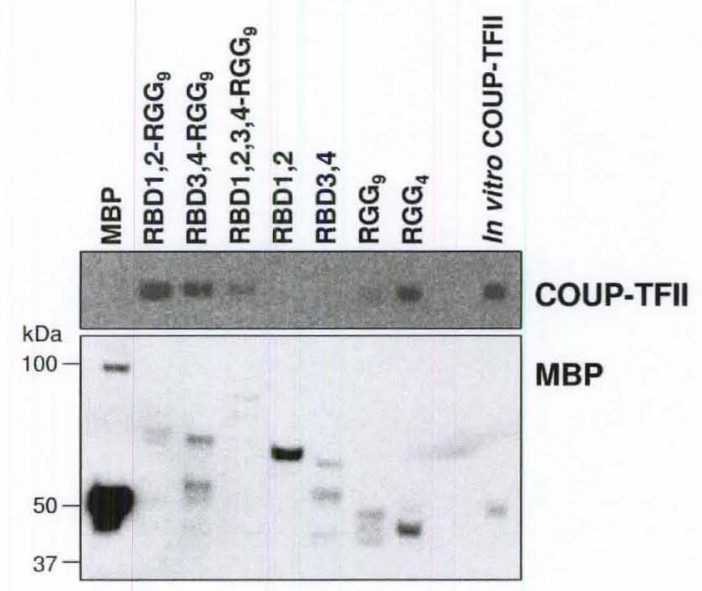

Figure 6. Endogenous nuclear nucleolin-COUP-TFII interaction in MCF-7 and T47D cells.

NE (200 $\mu$ g protein) from MCF-7 cells (A) and (400 $\mu$ g protein) from T47D (B) cells were immunoprecipitated with COUP-TFII antibody or with rabbit IgG (negative control), followed by western blot analysis for nucleolin and COUP-TFII. 5\% input NE serves as loading control. C, Immunofluorescent staining of endogenous COUP-TFII (green) and nucleolin (red) in the nuclei (Hoechst, blue) of MCF-7 cells. Merged images are shown at the right. Bar is $10 \mu \mathrm{m}$. D, schematic representation of the $\mathrm{N}$ - terminal maltose binding protein (MBP)-tagged recombinant nucleolin proteins used for MBP pull-down assays. MBP was fused to the N-termini of the RNA binding domains (RBD) and/or the arginine/glycine-rich domain (RGG) of nucleolin. E, In vitro transcribed/translated COUP-TFII was incubated with the MBP-nucleolin fragments or MBP. Interacting proteins were captured with amylose resin. Eluted proteins were probed for COUP-TFII (top) and MBP (bottom, control). 


\section{Direct interaction of COUP-TFII with the RGG domain of nucleolin}

To determine if COUP-TFII interacts directly with nucleolin and which domain(s) are involved, in vitro transcribed/translated COUP-TFII and MBP-tagged recombinant nucleolin polypeptides were incubated with an amylose affinity resin (Fig. 6D and E). The MBP fusion proteins contain the RNA binding domains (RBD1,2,3,4) and/or the arginine-glycine repeat (RGG). Nine RGG repeats are present in the C-terminus of nucleolin. Only these domains were investigated because the $\mathrm{N}$-terminal domain of nucleolin cannot be expressed in E. coli [247]. COUP-TFII was bound to all MBPtagged polypeptides containing the RGG domain but not with MBP-RBD1,2, MBPRBD3,4, or MBP alone. RGG9 appears to interact with COUP-TFII with weaker affinity compared to RGG4, perhaps because of the lower abundance of the MBP-RGG9 protein relative to MBP-RGG4. Overall, these results indicate that the C-terminal RGG domain is the minimal domain required for COUP-TFII-nucleolin interaction.

Immunohistochemical COUP-TFII and nucleolin staining in human breast tissue and tumor tissue microarrays

Nuclear COUP-TFII and nucleolin immunoreactivity were examined in two independent human breast tissue microarrays (TMAs, Fig. 7). In the TMAs from U.S. Biomax, significant differences in COUP-TFII staining were observed between TNM classes of tumor grades II $(d=-51.7, p=0.078)$ and II III $(d=-58.5, p=0.046)$ (Fig. 7C). COUP-TFII and nucleolin staining were correlated in invasive ductal carcinomas 
$\left(\varphi_{\mathrm{p}}=0.31, \mathrm{p}=0.0281 ; \varphi_{\mathrm{s}}=0.30, \mathrm{p}=0.0334\right)$. Normal breast tissue was also positive for COUP-TFII expression (Average H-score 127.5, SEM 5; data not shown). In a separate breast TMA [61], staining of a total of 332 patient tumors showed $~ 47 \%$ were positive for nuclear COUP-TFII (Table 3, Fig. 7F). There was a significant association between tumor grade and COUP-TFII with high grade tumors tending to be COUP-TFII negative (Table 3). COUP-TFII was also significantly positively associated with ER $\alpha$, SRC-1, PEA3, MMP2, and phospho-Src and negatively associated with HER2 (Table 3). High SRC-1 (NCOA1) was associated with a favorable response to TAM [258], a finding that corresponds to COUP-TFII's role in 4-OHT-medicated inhibition of breast cancer cell proliferation [116]. However, breast tumors from aromatase inhibitor-resistant patients show high expression of SRC-1 and a reduction in disease-free survival [259]; thus, the relationship between COUP-TFII and SRC-1 expression will require further investigation. SRC-1 and PEA3 synergistically activated COUP-TFII-promoter luciferase activity in transiently transfected HeLa cells [181]. PEA3 directly activates MMP2 transcription [244]; hence COUP-TFII may be correlated with MMP2 through the PEA3-COUP-TFII connection, although this hypothesis will require further analysis beyond the scope of the present study. The relationship between COUP-TFII and phospho-Src may be because activation of Src is part of the MAPK pathway that increases COUP-TFII expression [183]. According to Kaplan Meier, COUP-TFII did not associate with disease free survival in tamoxifen-treated patients $(p=0.4471, \mathrm{Fig} .7 \mathrm{H}$ and I). 

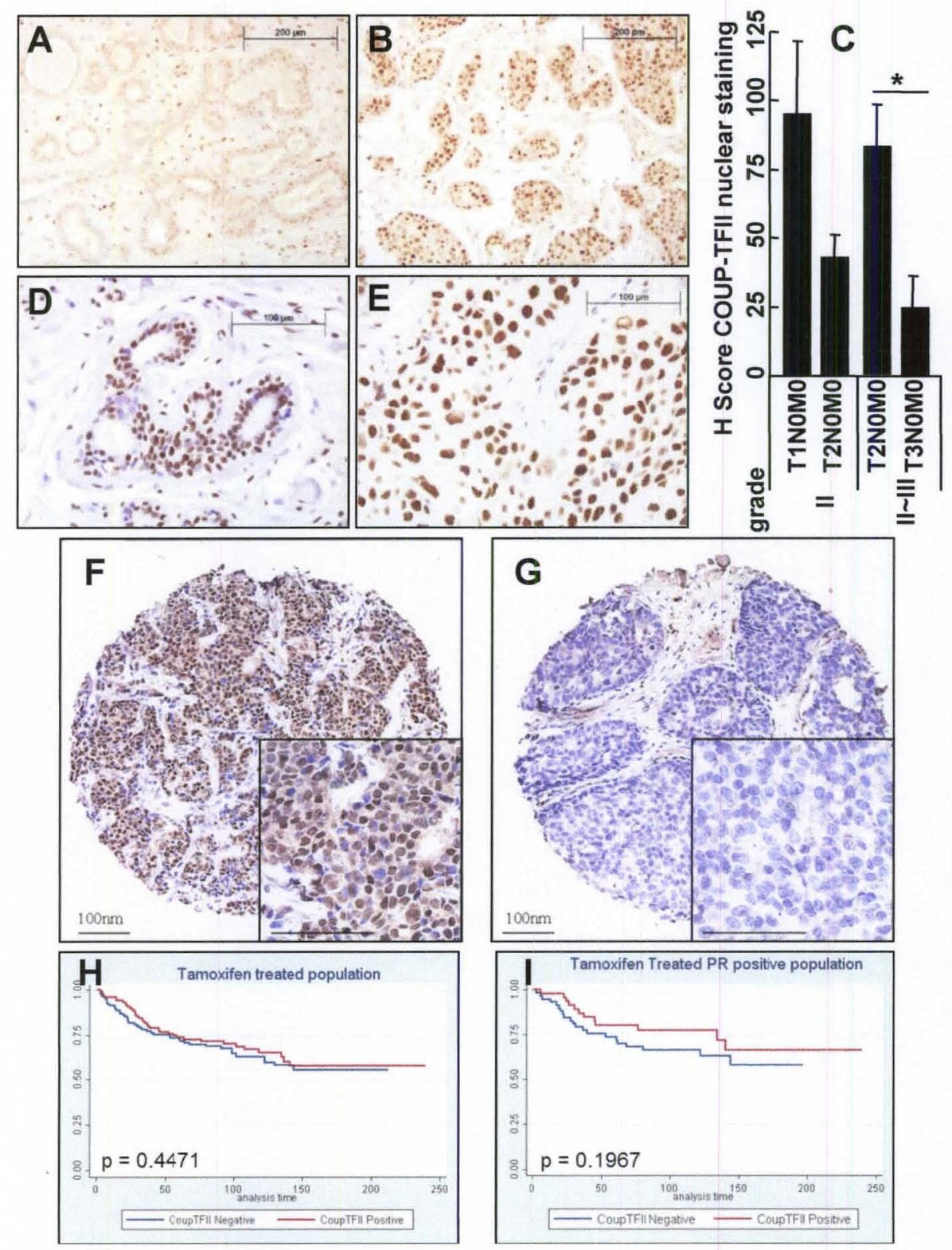

Figure 7. COUP-TFII and nucleolin in breast cancer tissue microarrays.

$\mathrm{A}$ and B, COUP-TFII immunostaining at 200x: A, benign breast tissue (H-score 30) and $\mathrm{B}$, invasive ductal carcinomas, grade 2 (H-score 153). Bar is $200 \mathrm{~mm}$. C, Average \pm SEM of H-score for nuclear COUP-TFII staining in ER $\alpha$-positive invasive ductal carcinomas by tumor grade. * significantly different from T2N0M0 (p<0.05). D and E, Nucleolin immunostaining at 400x: D, benign breast tissue (H-score 13) and E, invasive ductal carcinomas, grade 3 (H-score 151). Bar is $100 \mu \mathrm{m}$. F and G, immunohistochemical localization of COUP-TFII (100×, inset: $200 \times)$ on a tissue microarray constructed from archival tissue from 332 breast cancer patients [61] showing positively (F) and negatively (G) stained cores COUP-TFII at 200x, bar is $100 \mu \mathrm{m}$. H and I, Kaplan-Meier estimates of disease-free survival functions were computed, and the Wilcoxon test was used to compare survival curves. In addition, the Wilcoxon rank sum test was used to compare two medians. The data are not statistically significant. 
Table 3. COUP-TFII staining in breast tumor microarray.

Associated expression of COUP-TFII with ER $\alpha$, PR, HER2, SRC-1, AIB1, Pea3, AIB1, MMP2, and phospho-Src (psrc) staining in 560 human breast tumors [61,244]. Statistical analysis was performed using the Fisher's exact test, and a $\mathrm{P}$ value of $<0.05$ is considered to be significant (bold values).

\begin{tabular}{|c|c|c|c|}
\hline & $\begin{array}{l}\text { Total } \\
\text { Population } \\
n=321\end{array}$ & $\begin{array}{l}\text { \% COUPTFII } \\
\text { positive } \\
47 \% \\
\end{array}$ & $\begin{array}{l}\mathbf{p} \\
\text { value }\end{array}$ \\
\hline \multicolumn{4}{|l|}{ ER } \\
\hline neg & 99 & 34.3 & \multirow[b]{2}{*}{0.004} \\
\hline pos & 222 & 52.3 & \\
\hline \multicolumn{4}{|l|}{ PR } \\
\hline neg & 133 & 50.4 & \multirow[b]{2}{*}{0.339} \\
\hline pos & 146 & 44.5 & \\
\hline \multicolumn{4}{|l|}{ Her2 } \\
\hline neg & 267 & 50.2 & \multirow[b]{2}{*}{0.011} \\
\hline pos & 62 & 32.3 & \\
\hline \multicolumn{4}{|c|}{ Grade } \\
\hline 1 & 24 & 54.2 & \multirow[b]{3}{*}{0.003} \\
\hline 2 & 122 & 54.1 & \\
\hline 3 & 112 & 33.0 & \\
\hline \multicolumn{4}{|c|}{ Node } \\
\hline neg & 156 & 47.4 & \multirow[b]{2}{*}{0.824} \\
\hline pos & 167 & 46.1 & \\
\hline \multicolumn{4}{|c|}{ Recurrence } \\
\hline neg & 200 & 46.0 & \multirow[b]{2}{*}{0.822} \\
\hline pos & 132 & 47.7 & \\
\hline \multicolumn{4}{|c|}{ SRC-1 } \\
\hline neg & 214 & 41.6 & \multirow[b]{2}{*}{0.011} \\
\hline pos & 113 & 56.6 & \\
\hline \multicolumn{4}{|c|}{ AlB1 } \\
\hline neg & 88 & 25.0 & \multirow[b]{2}{*}{$<.001$} \\
\hline pos & 221 & 55.7 & \\
\hline \multicolumn{4}{|c|}{ Pea3 } \\
\hline neg & 120 & 35.8 & \multirow[b]{2}{*}{0.001} \\
\hline pos & 120 & 58.3 & \\
\hline \multicolumn{4}{|c|}{ MMP2 } \\
\hline neg & 29 & 20.7 & \multirow[b]{2}{*}{0.005} \\
\hline pos & 284 & 48.2 & \\
\hline \multicolumn{4}{|l|}{ psrc } \\
\hline neg & 226 & 42.0 & \multirow[b]{2}{*}{0.031} \\
\hline pos & 87 & 56.3 & \\
\hline
\end{tabular}


atRA enhances COUP-TFII binding to the RARB2 promoter

COUP-TFII is required for atRA- or 9cRA- induced $R A R B 2$ expression in breast cancer cells $[118,131]$ and binds the RARB2 promoter in electrophoretic mobility shift assays [118]. To examine COUP-TFII interaction with the RARB2 promoter, we first examined recruitment of endogenous COUP-TFII to the $R A R B 2$ promoter using the R\&D systems COUP-TFII antibody, but were unable to detect product in the PCR reactions (data not shown) likely due to lower COUP-TFII protein in MCF-7 cells compared to C3H10T1/2 CH3 mouse embryo mesenchymal cells that express high levels of COUPTFII [260]. To obviate this difficulty, MCF-7 cells were transfected with an empty vector or COUP-TFII-FLAG and ChIP was performed after FLAG IP. ChIP revealed for the first time that COUP-TFII binds the RARB2 promoter and atRA increased COUPTFII occupancy at the RARB2 promoter by $72 \%$ in MCF-7 cells (Fig. 8A; Appendix I, Fig. S5).

AS1411 inhibits COUP-TFII-stimulated RARB2 gene expression in MCF-7 and T47D breast cancer cells

Once establishing the presence of COUP-TFII at the promoter of its target gene $R A R B 2$, we sought to determine if nucleolin functions as a coactivator for COUP-TFIImediated $R A R B 2$ expression. RARB2 was increased in MCF-7 cells transfected with COUP-TFII and nucleolin overexpression potentiated the RARB2 induction in a concentration-dependent manner (Fig. 8B). Further, because the G-rich, G-quartet forming DNA aptamer AS1411 binds and reduces nucleolin activity [252,261], we hypothesized that AS1411 would inhibit COUP-TFII-stimulated RARB2 expression. 
AS1411 inhibited the COUP-TFII-induced increase in RARB2, while CRO (negative control) had no effect (Fig. 8C). Although nucleolin did not affect basal RARB2 expression, nucleolin abrogated the inhibition of $R A R B 2$ transcription by AS1411 (Fig. 8C). COUP-TFII also increased luciferase activity from a $R A R B$ gene promoter-reporter and AS1411 abrogated luciferase induction (Fig. 8D). 
A

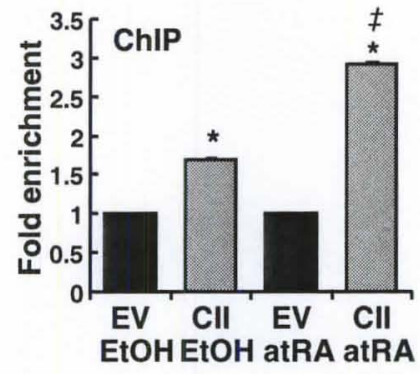

B

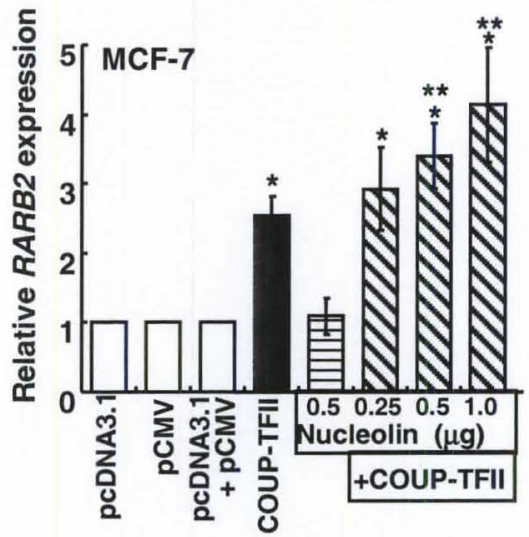

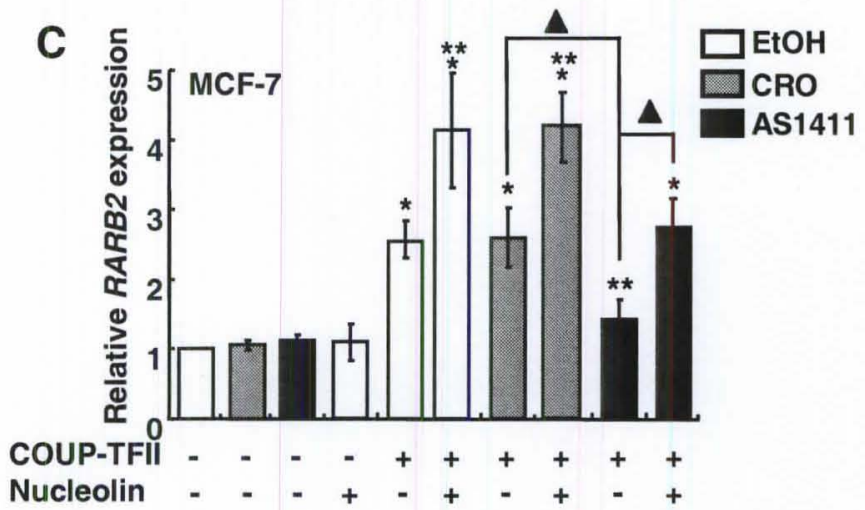

D

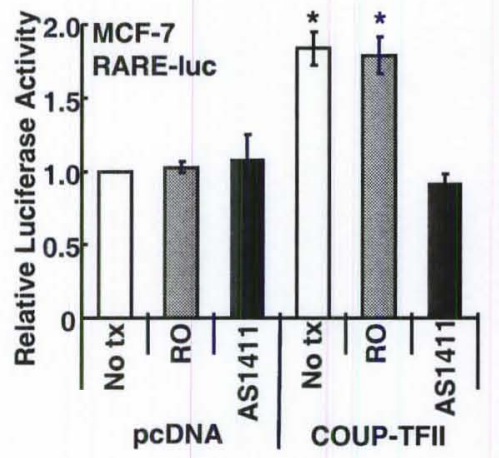

Figure 8. COUP-TFII increases RARß2 transcription in MCF-7 cells.

A, ChIP of COUP-TFII-FLAG to the RARB2 promoter in MCF-7 cells transfected with empty vector (EV) or COUP-TFII (CII) and treated with EtOH or $1 \mu \mathrm{M}$ atRA for $6 \mathrm{~h}$. * $\mathrm{P}<0.05$ versus $\mathrm{E}$ control, $\neq \mathrm{P}<0.05$ versus $\mathrm{CII}-\mathrm{EtOH}$. B-C, Cells were transfected with parental or expression plasmids for COUP-TFII or nucleolin for $24 \mathrm{~h}$ and were treated with EtOH, $10 \mu \mathrm{M}$ CRO or AS1411 for 24 h post-transfection. For C, cells were transfected with $0.5 \mu \mathrm{g}$ and $1 \mu \mathrm{g}$ of nucleolin and COUP-TFII expression vector, respectively. QRT-PCR was performed to determine $R A R B 2$ expression. Values are the average of 6 separate experiments \pm SEM. D, Cells were transfected with pcDNA or pcCOUP-TFII and treated with $10 \mu \mathrm{M}$ RO or AS1411 for $24 \mathrm{~h}$. Dual luciferase activity was expressed relative to the pcDNA-transfected, no-treatment control. Values are mean \pm S.E.M. of two separate experiments. For $\mathrm{B}-\mathrm{D}, * \mathrm{P}<0.05$ versus vector control, ** COUP-TFII alone, or $\boldsymbol{\Delta}$ between the indicated values. 
To be sure that any effect of AS1411 on RARB2 is not cell-line specific, MCF-7 and T47D cells with similar nucleolin expression (Appendix I, Fig. S6) were tested. T47D has $~ 40 \%$ lower COUP-TFII than MCF-7 (Appendix I, Fig. S6). Both atRA and 9cRA increased RARB2 expression in MCF-7 and T47D cells, with greater induction in T47D, and increased NR2F2 (COUP-TFII) expression in T47D (Fig. 9A-B).

Pretreatment of MCF-7 cells with AS1411, but not negative control CRO, reduced atRA-induced $R A R B 2$ expression (Fig. 9C). Transfection of MCF-7 cells with a nucleolin expression vector increased basal RARB2 except in AS1411-treated cells. Nucleolin and atRA additively increased RARB2 expression in MCF-7 cells and AS1411, but not $\mathrm{CRO}$, reduced RARB2 induction. Nucleolin significantly abrogated the inhibition of RARB2 expression by AS1411. COUP-TFII mRNA levels were not significantly reduced by AS1411 (Appendix I, Fig. S7). Neither ER antagonists ICI 182,780 nor 4OHT blocked atRA-induced $R A R B 2$ expression, indicating that ER is not involved in COUP-TFII-activated RARB2 expression (Fig. 9C). ICI and 4-OHT increased basal RARB2 in MCF-7, in agreement with $R A R B$-luciferase reporter activation in transfected MCF-7 cells [262].

Pretreatment of T47D cells with AS1411, but not CRO, reduced atRA-induced RARB2 expression and co-transfection with an expression vector for nucleolin significantly abrogated the inhibition of RARB2 expression by AS1411 (Fig. 9D). AS1411 had no effect on MCF-7 cell viability for the treatment times used in these experiments (Appendix I, Fig. S8), commensurate with previous findings that MCF-7 viability is inhibited only after $6 \mathrm{~d}$ of AS1411 treatment [261]. 
A

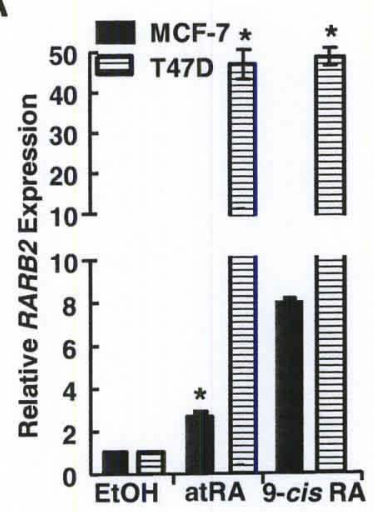

B

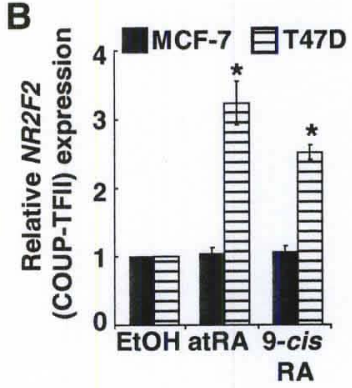

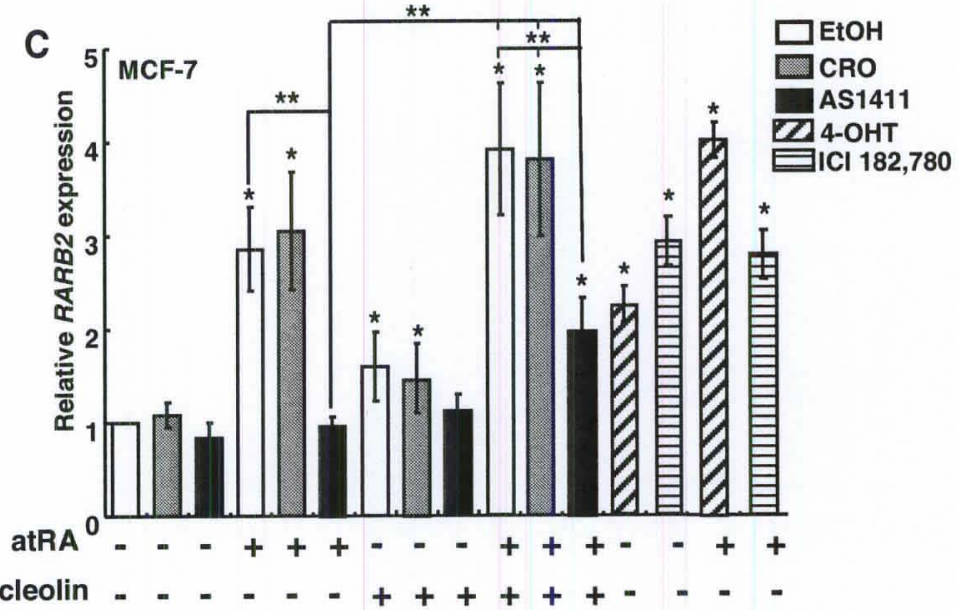

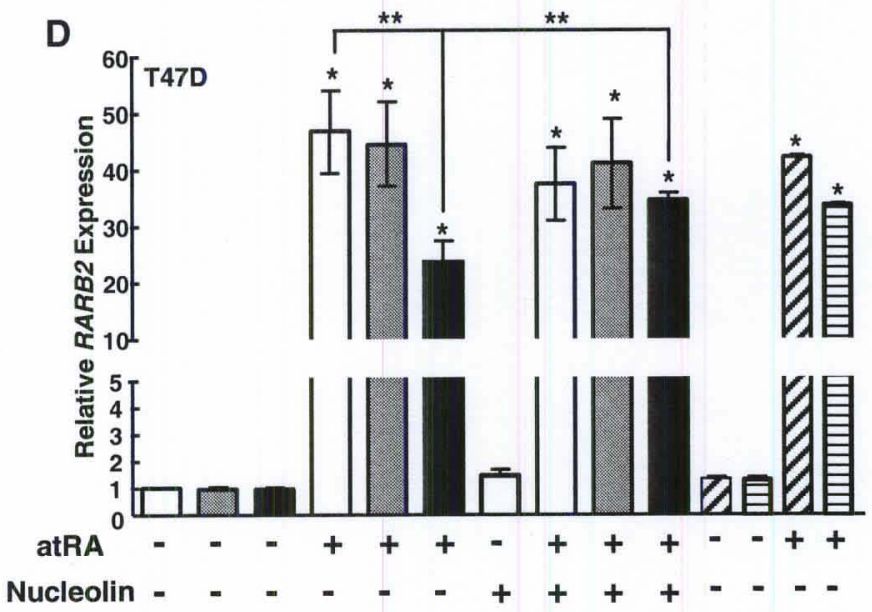

Figure 9. Regulation of RARß2 transcription.

$\mathrm{A}$ and B, QRT-PCR for RARB2 (B RAR 32 ) and NR2F2 (C COUP-TFII) in MCF-7 or T47D cells treated with EtOH, $1 \mu \mathrm{M}$ atRA, or $1 \mu \mathrm{M}$ 9-cis-RA for $24 \mathrm{~h}$. Values are the average of 3-5 separate experiments. * $\mathrm{P}<0.05$ versus EtOH. C, MCF-7 and D, T47D cells were transfected with $2 \mu \mathrm{g}$ pCMV-tag2 (-) or pCMV-tag2-nucleolin (+) for $24 \mathrm{~h}$ prior to $24 \mathrm{~h}$ treatment with $\mathrm{EtOH}, 10 \mu \mathrm{M} \mathrm{CRO}$, or $10 \mu \mathrm{M}$ AS1411. Where indicated, cells were treated with $1 \mu \mathrm{M}$ atRA, $100 \mathrm{nM} 4-\mathrm{OHT}$, or $100 \mathrm{nM}$ ICI 182,780 for $6 \mathrm{~h}$. QRT-PCR for RARB2 expression. Values are the average of 6 (MCF-7) and 4-10 (T47D) separate experiments \pm SEM. * $\mathrm{P}<0.05$ versus $\mathrm{EtOH}$ or $* *$ between the indicated values. 
RARB2 expression is inhibited by nucleolin or COUP-TFII knockdown

siNucleolin reduced nucleolin (NCL) mRNA by $37-58 \%$ and protein by $22-35 \%$ (Fig. 10A) in MCF-7 cells. In parallel, basal RARB2 was reduced 32-56\% (Fig. 10A). To determine if nucleolin knockdown inhibited atRA-induced $R A R B 2$, T47D cells were transfected with siNucleolin and untreated or treated with atRA (Fig. 10B). atRA had no effect on nucleolin knockdown (Fig. 10B). Analogous to the MCF-7 cells, siNucleolin reduced RARB2 mRNA (Fig. 10B). siNucleolin inhibited the atRA induction of RARB2 $\sim 36 \%$. Taken together, results from AS1411 and siNucleolin studies indicate a functional role for nucleolin as a coactivator of COUP-TFII-regulated atRA-induced RARB2 expression in T47D and MCF-7 cells.

siCOUP-TFII reduced COUP-TFII protein and, consequently, RARB2 transcription and protein (Fig. 10C). RAR $\beta 2$ stimulates retinoic acid receptor-induced gene 1 (RRIG1) transcription [263]. siCOUP-TFII reduced basal and atRA-induced RRIG1 expression. Transfection with COUP-TFII increased RRIG1. These data substantiate COUP-TFII's regulation of functional RAR $\beta 2$.

Effects of AS1411 on nucleolin-COUP-TFII nuclear interaction

AS1411 reduced the nuclear/cytoplasmic ratio of the nucleolin-interacting protein PRMT5 (Protein Arginine Methyltransferase 5) in DU145 prostate cancer cells [247]. AS1411 or CRO did not change nuclear COUP-TFII or nucleolin or cytosolic nucleolin in MCF-7 cells (Fig. 11A-B). There was no change in relative COUP-TFII-nucleolin interaction in MCF-7 cells treated with AS1411 (Fig. 11C- D). 
A
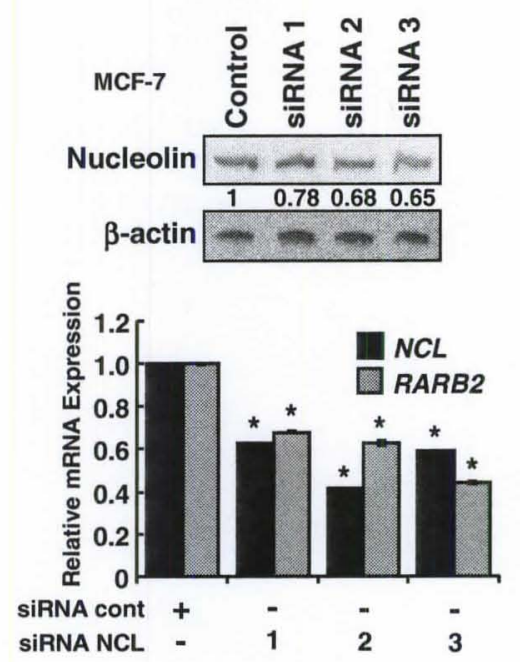

B

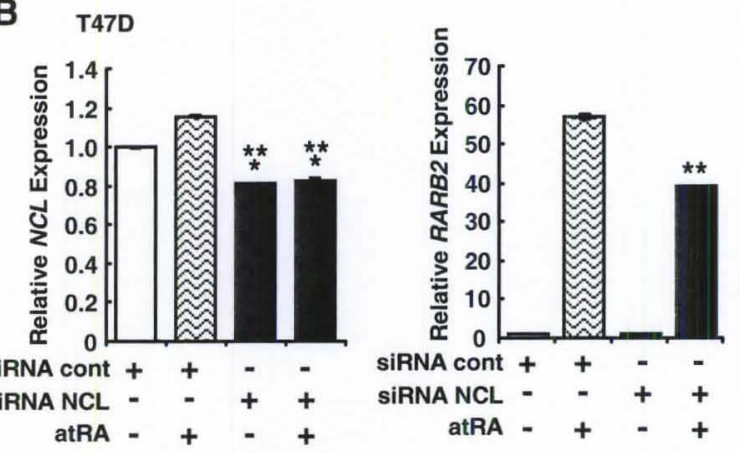

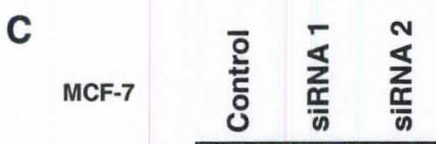
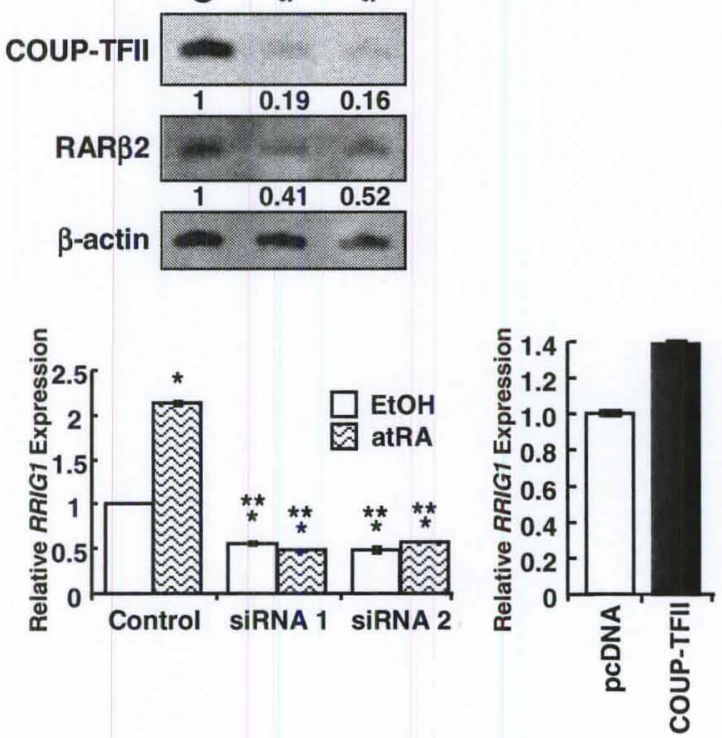

Figure 10. Reduction of COUP-TFII or nucleolin decreases RARß2 transcription in MCF-7 cells.

MCF-7 (A) and T47D (B) cells were transfected with control siRNA or an siRNA targeting nucleolin for $48 \mathrm{~h}$. T47D cells were treated with EtOH or $1 \mu \mathrm{M}$ atRA for $24 \mathrm{~h}$. QRT-PCR for nucleolin (NCL) and RARB2. Values are the average of triplicates. C, Western blot showing COUP-TFII and RAR $\beta 2$ expression after transfection with siCOUP-TFII. Values are relative to $\beta$-actin. MCF-7 were transfected with siControl or siCOUP-TFII for $48 \mathrm{~h}$ and treated with $1 \mu \mathrm{M}$ atRA for $6 \mathrm{~h}$. QRT-PCR was also performed for RRIG1. P $<0.001 *$ versus control or ** versus atRA. 
A
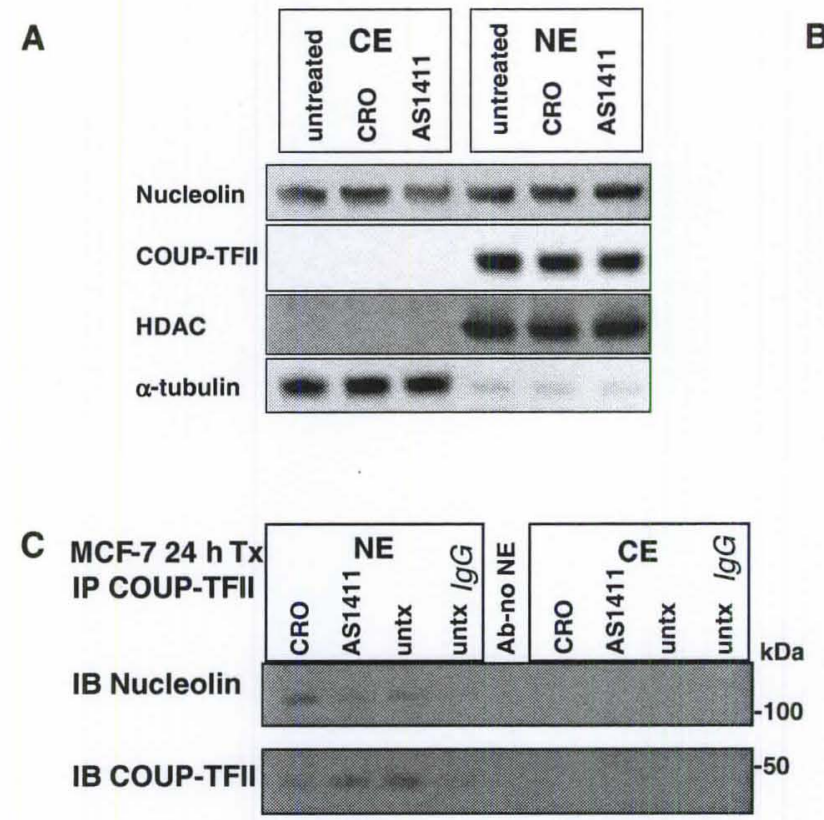
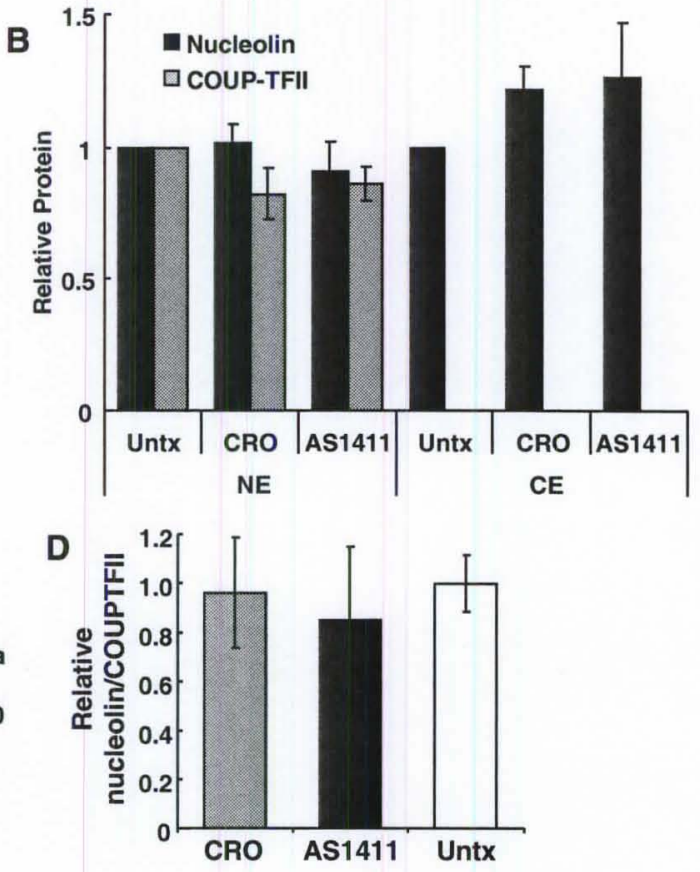

Figure 11. Effects of AS1411 on nuclear nucleolin-COUP-TFII interaction.

A, Representative western blots of CE and NE (30 $\mu \mathrm{g})$ from MCF-7 cells: untreated (untx) or treated with $10 \mu \mathrm{M}$ CRO or AS1411 for $24 \mathrm{~h}$ were probed for nucleolin or COUP-TFII , then stripped and reprobed for HDAC1 and $\alpha$-tubulin. B, Relative nucleolin and COUP-TFII expression (normalized to respective loading controls and untreated sample protein ratios were set to 1 for NE and CE). Bars are the mean \pm SEM of 4-8 separate experiments. C, NE or CE $(200 \mu \mathrm{g})$ from MCF-7 cells treated as above were IPed with COUP-TFII antibody or rabbit IgG. The Ab-no NE lane was a negative control: COUP-TFII antibody incubated with beads and buffer without NE. D, The ratio of nuclear nucleolin/COUP-TFII is the mean \pm SEM of 3 experiments. 


\section{DISCUSSION}

COUP-TFII plays an undefined role in breast cancer [116]. In this report, we identify nucleolin as a new functional partner for COUP-TFII. We have demonstrated for the first time that COUP-TFII binds nucleolin in vitro and in vivo. In breast tumors, nuclear nucleolin correlates with ER $\alpha$ and cell proliferation [264,265]. Here IHC staining revealed a correlation between nuclear COUP-TFII and nucleolin staining in invasive ductal carcinoma, a finding that reflects a previous report showing overexpression of COUP-TFII in A549 lung adenocarcinoma cells increased in vitro tumorigenicity and migration [211]. We demonstrate that nucleolin acts as a coactivator of endogenous COUP-TFII transcriptional activity for $R A R B 2$ in breast cancer cells. Similarly, nucleolin acted as a coregulator by interacting with c-Jun and Sp1 and increasing cytosolic phospholipase A2 (cPLA2) gene transcription [256].

We report a positive correlation between COUP-TFII and ER $\alpha, \mathrm{SRC}-1, \mathrm{Pea} 3$, MMP2, and phospho-Src expression, an inverse correlation of COUP-TFII with tumor grade and reduced COUP-TFII in ER $\alpha$-positive, invasive ductal carcinomas with increased TNM stage within tumor grades II and III and HER2 positivity. These data are in agreement with Oncomine analysis demonstrating $N R 2 F 2$ is higher in ER $\alpha+$ tumors and lower in metastatic breast tumors in microarray data [266] (Appendix I, Fig. S9). These observations indicate that COUP TFII may play a role in "phenotype maintenance" and that its function may be restricted to the luminal breast cancer subtypes. We speculate that the precise role of COUP-TFII in breast cancer depends on cellular context, which is consistent with the role of other nuclear receptors in breast cancer [267], and remains to be fully elucidated. 
Other COUP-TFII-interacting proteins identified here include PARP-1, which also interacts with nucleolin [268], TOPOII $\beta$, involved in transcriptional activation by NRs [269,270], and DNA topoisomerase I (TopoI), which localizes to active transcription sites [271]. Reflecting its NR chaperone role and interaction with COUP-TFI [114], Hsp70 interacted with COUP-TFII. Other COUP-TFII-interacting proteins including hnRNP A2/B1, RPS20, RPL15, and RPL21 were also identified as binding with nucleolin to a c-myc G-quadruplex affinity column [272].

Nucleolin is a key target of the anticancer aptamer AS1411, although AS1411 interacts with other proteins, e.g., NEMO to inhibit NFKB activation and PARP-1 [252]. AS1411 has pleiotropic effects on nucleolin, e.g., inhibiting nucleolin binding to the AUrich element in the 3' UTR of BCL2 in MCF-7 cells causing apoptosis [273] and stimulating macropinocytosis [251]. AS1411 is used to functionally inhibit nucleolin [274]. Here, AS1411 and siNucleolin reduced COUP-TFII-induced RARB2 expression in MCF-7 and T47D cells and cotransfection with nucleolin reduced AS1411-inhibition. AS1411 did not alter nuclear COUP-TFII-nucleolin interaction, indicating that the mechanism for AS1411 inhibition of RARB2 expression is independent of reducing nuclear COUP-TFII protein, a result different from AS1411 reducing nuclear PRMT5 in DU145 cells [247]. The inhibition of atRA- and COUP-TFII- regulated RARB2 expression by AS1411 may also be independent of its effect on nucleolin and may indicate a potential adverse 'side effect' of AS1411 that may be a concern if this drug is used for breast cancer therapy.

In conclusion, COUP-TFII interacting proteins were identified in MCF-7 breast cancer cells. Endogenous COUP-TFII and nucleolin interact in both MCF-7 and T47D 
luminal breast cancer cells. A coregulatory role for nucleolin in COUP-TFII-mediated RARB2 transcription was described (Fig. 12). Retinoids, e.g. 9cRA and atRA, and RAR $\beta$ have long been associated with tumor suppressive properties such as reduced cell proliferation, inflammation, and solid tumor formation, as well as enhanced apoptosis (reviewed in [275]). RAR $\beta 2$ expression is reduced in breast tumors and restoration of RAR $\beta 2$ expression increases sensitivity to tumor growth inhibition by retinoids [276]. Ours is the first demonstration that atRA increased COUP-TFII-RAR $\beta 2$ promoter interaction by ChIP. Since serum carotenoids levels are inversely associated with breast cancer risk in women with high mammographic density [277], the increase in $R A R B 2$ in response to COUP-TFII-nucleolin interaction is consistent with a role for COUP-TFII in phenotype maintenance. In agreement with a previous report [118], we observed a much higher induction of RARB2 in T47D in response to atRA compared to MCF-7 despite, as newly reported here, lower COUP-TFII expression. These data indicate that other mechanisms are also involved. COUP-TFII protein staining in TMAs correlates with ER $\alpha$ expression and an inverse correlation of COUP-TFII with tumor grade in ER $\alpha$ positive, invasive ductal carcinomas was detected, a finding that correlates with reduced COUP-TFII expression in endocrine-resistant breast cancer cells [116]. Together, these data suggest that COUP-TFII may be important in differentiated ER $\alpha$-expressing, retinoid-responsive, epithelial breast cancer cells and that reduced COUP-TFII leads to tumor advancement, including endocrine resistance. 


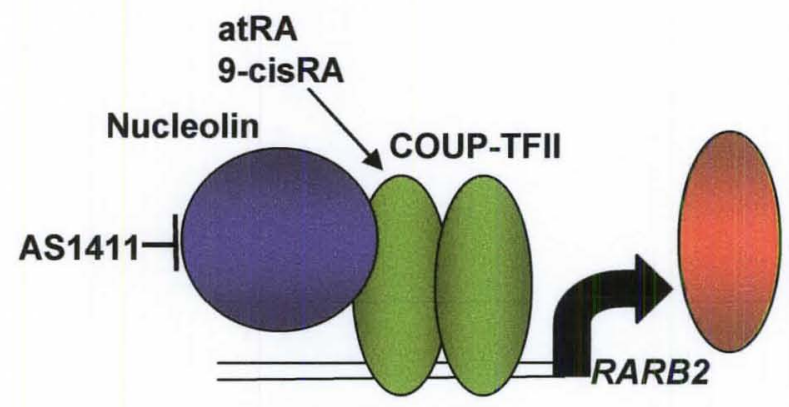

Figure 12. Model of nucleolin-COUP-TFII interaction and upregulation of RARB2 expression.

COUP-TFII binds its response elements as either a homodimer or as a heterodimer with RXR [107]. Previous reports demonstrated that 1) 9cisRA and atRA bind COUP-TFII and increase COUP-TFII transcriptional activity [120]; 2) Nucleolin acts as a transcriptional coregulator by interacting with cJun and Sp1 [256]; 3) COUP-TFI and COUP-TFII increase RARB2 expression in cooperation with RAR $\alpha$ and CBP [118]. Here we demonstrated that 1) nucleolin interacts directly with nuclear COUP-TFII; 2) atRA and 9-cisRA increased RARB2 mRNA; 3) AS1411, used as a functional inhibitor of nucleolin [252,274], inhibited COUP-TFII-upregulation of RARB2 gene transcription; 4) siRNA knockdown of nucleolin reduces induction of RARB2 and reduced RAR $\beta 2$ protein. 


\section{CHAPTER IV - COUP-TFII INHIBITS NFאB ACTIVATION IN BREAST CANCER CELLS AND RESTORES ENDOCRINE SENSITIVITY}

\section{INTRODUCTION}

Selective estrogen receptor modulators (SERMs) such as tamoxifen (TAM) exert antiproliferative effects on breast cancer by competing with estrogens for binding to estrogen receptor $\alpha(\mathrm{ER} \alpha)$, leading to an inhibition of downstream genes, including those involved in breast cancer proliferation. Fulvestrant is a selective ER $\alpha$ downregulator (SERD) because it causes proteasomal degradation of $\mathrm{ER} \alpha$ in addition to its SERM-like ability to antagonize estrogen-activated $E R \alpha$ transactivation [278]. TAM inhibits cell cycle progression and induces apoptosis $[45,46]$. TAM has greatly increased the survival rate of breast cancer patients since its initial FDA approval in 1977, resulting in a $31 \%$ reduction in annual death rate [48,279]. Despite the initial efficacy of SERM and aromatase inhibitor treatment, approximately $40 \%$ of patients relapse and die from metastatic disease because the cancer cells become refractory to endocrine therapies [56].

The mechanisms for acquired endocrine resistance are complex and a better understanding of maintenance of endocrine sensitivity is clearly needed. Reduced expression of the orphan nuclear receptor chicken ovalbumin upstream promoter transcription factor II (COUP-TFII) has a demonstrated role in acquired endocrine 
resistance in a breast cancer cell line model of human disease progression [116]. Restoration of COUP-TFII expression, which is reduced in endocrine-resistant breast cancer cell lines, inhibited cell proliferation and motility and increased apoptosis in TAM and fulvestrant-treated endocrine-resistant LCC9 and LY2 breast cancer cells [116]. Conversely, inhibition of COUP-TFII expression caused MCF-7 cells to become resistant to SERM and fulvestrant inhibition of proliferation [116]. A mechanism for the involvement of COUP-TFII in maintenance of endocrine sensitivity has not yet been determined. Since COUP-TFII acts as either a transcriptional activator or repressor in a gene- and cell-specific manner [226], the mechanism of action may involve modulation of specific target genes including tumor suppressors and oncogenes.

The NFkB family of dimeric transcription factors has important functions related to immune and inflammatory responses, and recent studies have demonstrated NFKB activation plays a role in acquired endocrine-resistant breast cancer $[87,88,280]$. Inactive $\mathrm{NF \kappa B}$ is located in the cytoplasm bound to IKB, the inhibitor of NFKB. Also present in the cytoplasm is the IKK complex containing the regulatory subunit NEMO (nuclear factor- $\mathrm{\kappa B}$ essential modulator or IKK $\gamma$ ) and the catalytic subunits IKK $\alpha$ and $\operatorname{IKK} \beta$. Upon activation by a variety of stimuli, the IKK complex phosphorylates the inhibitor ІкB leading to its degradation, freeing the now activated NFאB to translocate to the nucleus to regulate target gene transcription. Because NFKB activation results in expression of genes that lead to anti-apoptotic and pro-proliferative activities, increased NFKB signaling promotes cancer cell survival [281].

Dysregulation of the NFKB subunits p65 (RELA), RelB (RELB), p50 (NFKB1), p52 (NFKB2), and c-Rel (REL) results in altered activation of the NFKB pathway in 
breast cancer. Increased expression of p50/NFKB1, p52/NFкB2, and c-Rel was detected in breast tumors compared to adjacent normal tissue [282]. Elevated p50/NFKB1-DNA binding correlated with metastatic relapse and reduced disease-free survival in patients with ER $\alpha$-positive tumors [283]. An increase in NFKB activity has been reported in endocrine-resistant breast cancer cells $[87,280,284]$. The amount of RelA/p65 protein was also elevated [87]. The expression and DNA binding of p50 and p65 are also enhanced in LCC1 estrogen-independent, TAM-sensitive breast cancer cells compared to MCF-7 estrogen-dependent cells, further demonstrating a role for NFKB in the pathway to endocrine resistance [93].

Since COUP-TFII expression is decreased in endocrine-resistant breast cancer cells, we hypothesized COUP-TFII may play a part in the normal suppression of NFKB activity in endocrine-sensitive breast cancer cells by regulating the transcription of components of the NFKB pathway. In this study, we observed that COUP-TFII overexpression inhibited NFKB activity in LCC9 endocrine-resistant breast cancer cells. This suppression of the NFKB pathway resulted in reduced expression of downstream $\mathrm{NF \kappa B}$ target genes as well as NFKB subunits and increased sensitivity to 4hydroxytamoxifen (4-OHT) treatment. Our results provide a mechanism by which COUP-TFII maintains endocrine sensitivity by suppressing NFKB expression and activity in breast cancer cells.

\section{MATERIALS AND METHODS}

\section{Chemicals}


4-hydroxytamoxifen (4-OHT) and BMS-345541 were purchased from SigmaAldrich (St. Louis, MO). Tumor necrosis factor $\alpha$ (TNF $\alpha)$ was purchased from PeproTech (Rocky Hill, NJ).

\section{Antibodies}

The following antibodies were purchased: monoclonal (mAb) anti-human COUPTFII (R\&D systems, Minneapolis, MN); polyclonal RelA/p65, polyclonal and mAb RelB, and mAb NFkB1 p105/p50 (Santa Cruz Biotechnology, Santa Cruz, CA);

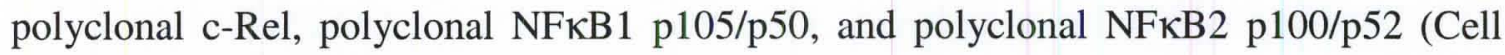
Signaling, Danvers, MA), $\beta$-actin (Sigma-Aldrich). HRP-conjugated secondary antibodies were from GE Healthcare (Piscataway, NJ).

\section{Cell culture and transient transfection}

MCF-7 cells were purchased from ATCC and used at passage $<10$. LCC9 cells were kindly provided by Dr. Robert Clarke [285]. MCF-7 and LCC9 cells were maintained as in [116]. Cells were transiently transfected as indicated in figure legends using FuGENE HD (Roche, Indianapolis, IN) as per the manufacturer's protocol.

\section{Luciferase assay}

To analyze NFאB activity, MCF-7 and LCC9 cells were transiently transfected with pGL4.32[luc2P/NF-אB-RE/Hygro] (Promega, Madison, WI) containing five copies of a NFkB response element, pGL4-hRluc-TK (Renilla, Promega), and pcDNA3.1 or pcDNA3.1-mCOUP-TFII (kindly provided by Drs. Sophia and Ming-Jer Tsai [246]) for 
$48 \mathrm{~h}$ before performing dual luciferase assay (Promega). Where indicated, cotransfection was performed with plasmids for CBP (kindly provided by Dr. Margarita HadzopoulouCladaras [286]), SRC-1 (kindly provided by Dr. Bert W. O’Malley [287]), GRIP1/SRC2, or ACTR/SRC-3 (kindly provided by Dr. Michael Stallcup [288]).

\section{$N F K B$ pathway $P C R$ array}

LCC9 cells were transfected with pcDNA3.1 or pcDNA3.1-mCOUP-TFII for 24 $\mathrm{h}$ before treating with $10 \mathrm{ng} / \mathrm{ml} \mathrm{TNF} \alpha$ for $6 \mathrm{~h}$. RNA was isolated using RNeasy (Qiagen, Valencia, CA) and cDNA was prepared using $\mathrm{RT}^{2}$ First Strand Kit (SABiosciences/Qiagen, Valencia, CA). Human NFאB Signaling Pathway PCR Array (SABiosciences/Qiagen) was run according to manufacturer's instructions.

Electrophoretic mobility shift assay (EMSA)

MCF-7 and LCC9 cells were transfected with pcDNA3.1 or pcDNA3.1-mCOUPTFII for $48 \mathrm{~h}$ followed by treatment with $10 \mathrm{ng} / \mathrm{ml}$ TNF $\alpha$ for $6 \mathrm{~h}$. Following centrifugation, cells were resuspended in $10 \mathrm{mM}$ HEPES, $1.5 \mathrm{mM} \mathrm{MgCl} 2,10 \mathrm{mM} \mathrm{KCl}, 1$ $\mathrm{mM}$ dithiothreitol (DTT, Sigma-Aldrich), 1X protease and phosphatase inhibitors (Roche). The cells were lysed with a 27 gauge syringe and centrifuged to obtain cytosolic extract. The resulting nuclear pellet was resuspended in EMSA buffer (10 mM Tris $\mathrm{HCl}, 150 \mathrm{mM} \mathrm{KCl}, 0.5 \mathrm{mM}$ EDTA, 0.1\% Triton-X 100, 12.5\% glycerol, $\mathrm{pH} 7.9$ (Sigma-Aldrich), $1 \mathrm{mM}$ DTT, $1 \mathrm{X}$ protease and phosphatase inhibitor (Roche)) and sonicated to prepare nuclear extract (NE). 
NFkB sense (5'-AGT TGA GGG GAC TTT CCC AGG C-3') and antisense (5'GCC TGG GAA AGT CCC CTC AAC T-3') oligonucleotides (Integrated DNA Technologies, Coralville, IA) were annealed and labeled with $\left[{ }^{32} \mathrm{P}\right]-\gamma \mathrm{ATP}$ (PerkinElmer, Waltham, MA) using T4 polynucleotide kinase (Promega) to prepare labeled DNA probe. The $\left[{ }^{32} \mathrm{P}\right] \mathrm{NF} \kappa \mathrm{B}$ response element (RE) probe was purified using the QIAquick Nucleotide Removal Kit (Qiagen).

25 ug NE from the indicated cells was incubated with poly-dIdC (Sigma-Aldrich), $\left[{ }^{32} \mathrm{P}\right] \mathrm{NF}$ KB-RE (labeled probe), and EMSA buffer for $1 \mathrm{~h}$ before separating on a $4 \%$ nondenaturing polyacrylamide gel (Bio-Rad, Hercules, CA). For in vitro binding assay, in vitro transcribed/translated COUP-TFII was prepared as described in [115] and incubated in increasing concentrations with NE from LCC9 cells treated with $10 \mathrm{ng} / \mathrm{ml}$ TNF $\alpha$ for 6 h.

\section{$N F \kappa B$ family transcriptional assay}

MCF-7 or LCC9 cells were transfected with pcDNA3.1 or pcDNA3.1-COUPTFII for $48 \mathrm{~h}$ and treated with $10 \mathrm{ng} / \mathrm{ml} \mathrm{TNF} \alpha$ for $6 \mathrm{~h}$ before preparing NE. $15 \mu \mathrm{g} \mathrm{NE}$ was used for TransAM NFkB assays (Active Motif, Carlsbad, CA) according to the manufacturer's protocol. Briefly, NE was added to 8 well strips containing immobilized

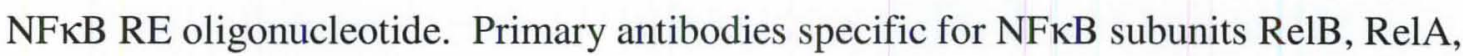
p50, p52, and c-Rel were used to identify subunit-specific NFKB activation. Secondary HRP-conjugated antibody and provided buffers provided colorimetric quantitation via spectrophotometric analysis at $450 \mathrm{~nm}$. 


\section{Coimmunoprecipitation}

Nuclear extracts were prepared from MCF-7 cells as in [115]. Dynabeads protein A (Life Technologies, Carlsbad, CA) were incubated with COUP-TFII antibody (Abcam, Cambridge, MA) or negative control IgG (Santa Cruz) for 30 minutes prior to the addition of $400 \mu \mathrm{g}$ MCF-7 $\mathrm{NE}$ for $4 \mathrm{~h}$ at $4^{\circ} \mathrm{C}$ with rotation. Following washes, protein was eluted with Elution Buffer (Life Technologies, Carlsbad, CA) and pH was adjusted with $1 \mathrm{M}$ TrisHCl.

\section{Cell proliferation assay}

MCF-7 and LCC9 cells were grown for $48 \mathrm{~h}$ in medium containing 5\% charcoalstripped fetal bovine serum (CSS) and transfected with pcDNA or pcDNA-mCOUP-TFII for $24 \mathrm{~h}$ before treatment with $1 \mu \mathrm{M}$ 4-OHT, $10 \mathrm{ng} / \mathrm{ml} \mathrm{TNF} \alpha$, and 0.01-5 $\mu \mathrm{M}$ BMS345541 in DMSO for 5 days. Cell proliferation was assayed using CellTiter AQueous One Solution Cell Proliferation Assay (MTS; Promega).

RNA Isolation, RT-PCR and Quantitative Real-Time-PCR (QRT-PCR)

RNA isolation, RT-PCR, and QRT-PCR were performed as in [115]. The following SYBR green primers were used for QRT-PCR analysis, relative to GAPDH control: RELB [289], REL, NFKB1 and NFKB2 [290], IL6 [291], A20/TNFAIP3 [292], ICAM1 [293], CCL2 [294], NR2F2 and GAPDH [183], Nr2f2 (Forward 5'CCCCCATAGATATGGCAATGGTAGTCAGCACG-3', Reverse 5'TTGATTTATTTATTGAATTGCCATATATGGCC-3'). p65/RELA was measured using TaqMan Gene Expression Assay (Applied Biosystems) relative to $18 \mathrm{~S}$ control. 


\section{Microarray analysis}

Published microarray data was obtained from Gene Expression Omnibus (GEO) (dataset GSE17705) consisting of $298 \mathrm{ER} \alpha+$ patients treated with tamoxifen for 5 years and analyzed to assess the correlation between COUP-TFII (NR2F2) expression and NFאB subunit (NFKB1, NFKB2, REL, RELA, RELB) and target gene (ICAM1, IL6, $C C L 2, T N F A I P 3)$ expression. Gene expression data was $\log 2$ transformed and quantile normalized prior to analysis. Correlation analysis was performed using the Spearman Rho $(\rho S)$ statistic to assess whether $N R 2 F 2$ expression was inversely correlated with NFKB subunit/target genes.

Statistical analysis

Statistical analyses were performed as in Chapter III [115].

\section{RESULTS}

COUP-TFII suppresses NF $K B$ activity

To determine if NFKB activity is differentially regulated by COUP-TFII in endocrine-sensitive versus -resistant cells, MCF-7 (sensitive) and LCC9 (resistant) cells were transfected with a luciferase reporter containing five tandem repeats of a NFKB responsive element as well as pcDNA3.1 parental plasmid or pcDNA3.1-COUP-TFII (Fig. 13A). Basal NFKB activity in LCC9 is $\sim 5$ fold higher than MCF-7 cells. When treated with TNF $\alpha$, a dramatic induction of NFKB activity is observed in LCC9 but not 
MCF-7 cells. A statistically significant, dose-dependent decrease in the TNF $\alpha$-induced NFKB activation occurs in LCC9 cells upon transfection with increasing amounts of COUP-TFII. These data demonstrate that COUP-TFII suppresses NFאB activity in endocrine-resistant breast cancer cells. The expression of NFkB subunits NFKB2, REL, and RELB was higher in LCC9 compared to MCF-7 cells (Fig. 13B), accounting for the higher NFאB activity in LCC9 cells. 
A

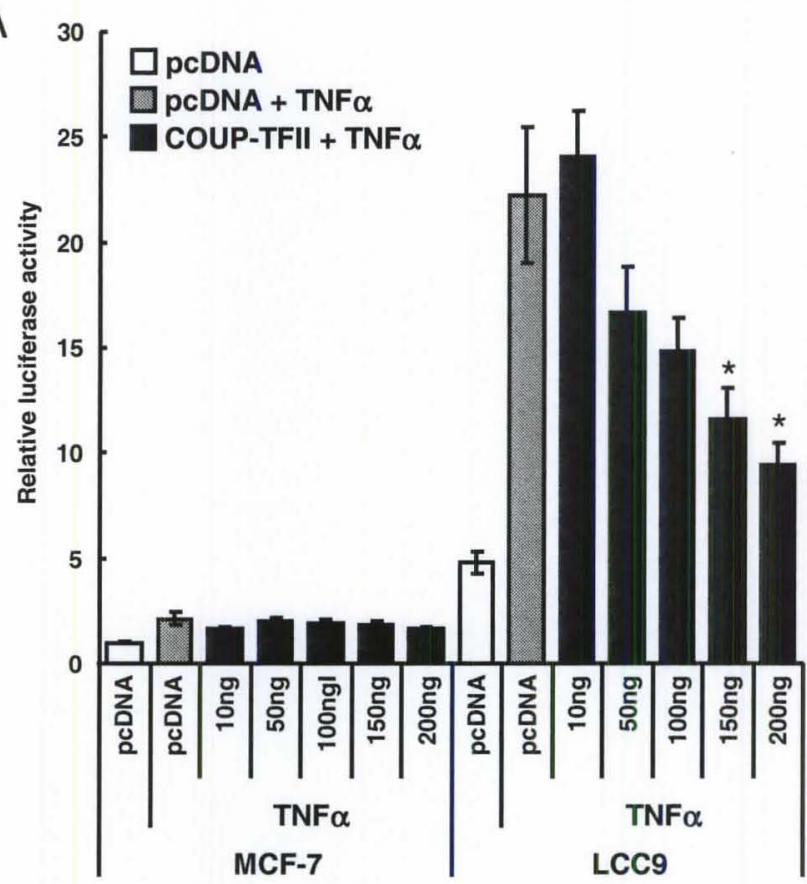

B

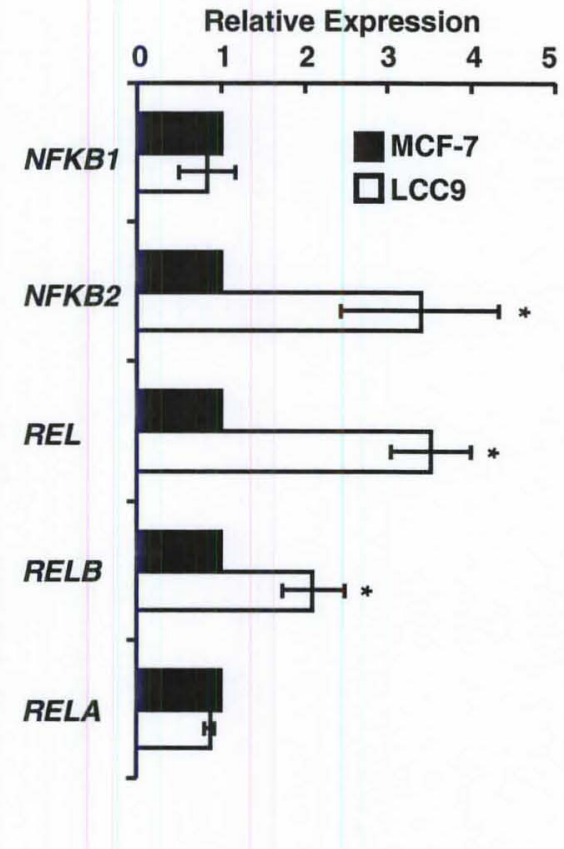

Figure 13. COUP-TFII suppresses NFkB activity in LCC9 cells.

(A) MCF-7 and LCC9 cells were transfected with a NFKB luciferase reporter and increasing concentrations of pcDNA3.1 or pcDNA3.1-COUP-TFII for $48 \mathrm{~h}$ and treated with $10 \mathrm{ng} / \mathrm{ml} \mathrm{TNF} \alpha$ for $6 \mathrm{~h}$ before performing dual luciferase assay (Promega). Firefly luciferase values are shown relative to protein concentration for a representative experiment of quadruplicate values \pm SEM. * Significantly different $\mathrm{P}<0.05$ from pcDNA+TNF $\alpha$ in LCC9. (B) Comparison of NFKB subunit mRNA expression in MCF-7 versus LCC9 cells. Values are the mean of 4-8 separate experiments \pm SEM. *: $\mathrm{P}<0.05$ versus MCF-7. 
Identification of COUP-TFII effect on NF KB pathway

An NFKB pathway PCR array was used to determine the effect of COUP-TFII on the NFKB pathway in LCC9 endocrine-resistant cells treated with TNF $\alpha$ using a 3-fold cutoff (Fig. 14A). As displayed in the resulting heatmap, a general decrease in the expression of NFKB pathway genes was detected upon COUP-TFII overexpression (Fig. 14B). Many genes suppressed by COUP-TFII greater than 3-fold were NFKB responsive genes, e.g., CCL2, IL6, LTA, ICAM1, and TNFAIP3, as well as NFKB subunits $(R E L B)$, reflecting a role for COUP-TFII in downregulation of the NFKB pathway. These genes were selected for further analysis.

COUP-TFII suppresses NF $K B$ target gene expression

To extend and confirm the results from the NFKB pathway PCR array, MCF-7 and LCC9 cells were transfected with pcDNA3.1 or pcDNA3.1-COUP-TFII and treated with TNF $\alpha$ to determine the effect of COUP-TFII on endogenous NFKB target gene expression. COUP-TFII expression following transient transfection was verified via western blot (Appendix I, Fig. S10). Four NFkB target genes with known roles in breast cancer progression were examined through QRT-PCR to validate the results from the NFKB pathway PCR array: interleukin 6 (IL6), intercellular adhesion molecule 1 (ICAM1), TNF $\alpha$-induced protein 3 (A20, TNFAIP3), and chemokine (C-C motif) ligand 2 (CCL2) (Fig. 15). TNFo increased the expression of IL6, ICAM1, TNFAIP3, and CCL2 in both MCF-7 and LCC9 cells (Fig 15A-D). COUP-TFII overexpression reduced the basal expression of IL6 and CCL2 in LCC9 cells (Fig. 15A and D) and ICAMI and 
TNFAIP3 in both MCF-7 and LCC9 cells (Fig. 15B and C). The TNF $\alpha$-induction of IL6 and ICAMI was reduced by COUP-TFII in LCC9 cells (Fig. 15A and B), while the TNFo-induction of TNFAIP3 and CCL2 was reduced in MCF-7 cells (Fig. 15C and D). The suppression of IL6, ICAM1, TNFAIP3, and CCL2 expression by COUP-TFII is in agreement with the data from the NFKB PCR array. 

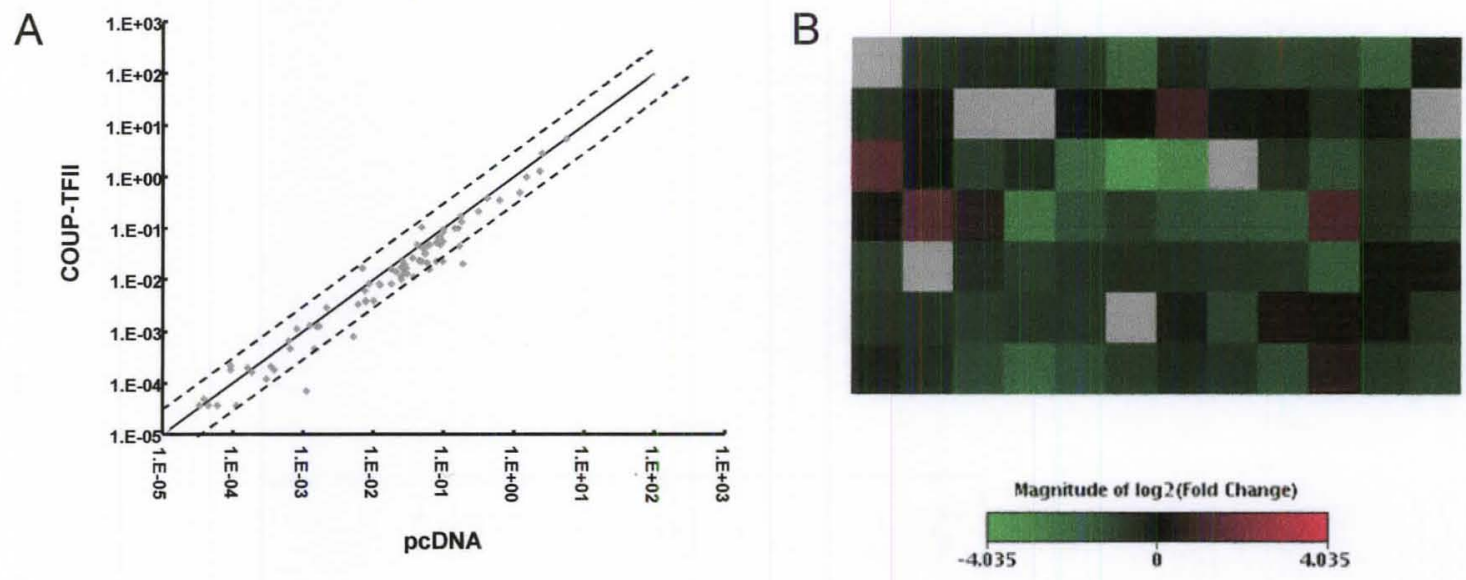

Figure 14. COUP-TFII inhibits expression of multiple genes in the NFKB pathway. LCC9 cells were transfected with pcDNA3.1 or pcDNA3.1-COUP-TFII for $24 \mathrm{~h}$ and treated with $10 \mathrm{ng} / \mathrm{ml} \mathrm{TNF} \alpha$ for $6 \mathrm{~h}$ before preparing RNA and cDNA. (A) SABiosciences NFKB Pathway Array was used to identify genes with expression altered greater than 3-fold by addition of COUP-TFII. (B) Heat map displaying fold change of individual genes in the array. 

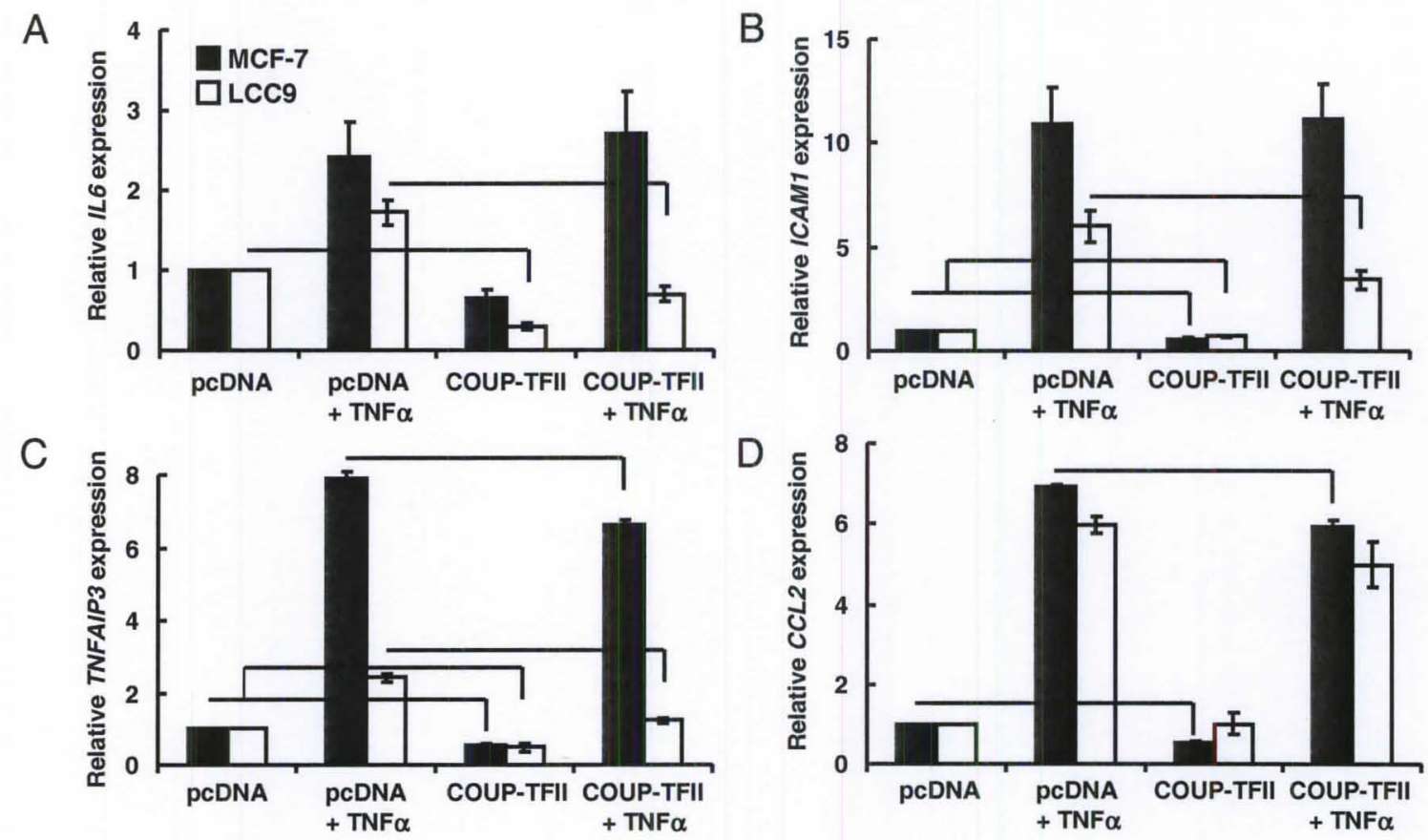

Figure 15. COUP-TFII inhibits expression of NFkB target genes.

MCF-7 and LCC9 cells were transfected with pcDNA3.1 or pcDNA3.1-COUP-TFII for $48 \mathrm{~h}$ and treated with $10 \mathrm{ng} / \mathrm{ml} \mathrm{TNF} \alpha$ for $6 \mathrm{~h}$ before preparing RNA and cDNA. mRNA expression of NFKB target genes identified in the NFKB pathway array (A. IL6, B. ICAM1, C. TNFAIP3, D. CCL2) was analyzed by QRT-PCR. Note that relative expression of each gene was normalized to pcDNA-transfected, control treated cells within each cell line. Values are the mean of 2-7 separate experiments \pm SEM. Bars indicate $\mathrm{p}<0.05$ between the indicated samples to indicate effect of COUP-TFII on basal or TNF $\alpha$-induced gene expression in each cell line. 
COUP-TFII suppresses NF KB subunit gene expression

$R E L B$ expression was decreased by COUP-TFII overexpression in the NFKB pathway PCR array. Increased NFKB subunit expression has been reported in several lineages of endocrine-resistant breast cancer cells $[87,88,91]$, but correlation with COUPTFII expression has not yet been examined. Consistent with these reports, we observed higher $N F K B 2$, REL, and RELB expression in LCC9 versus MCF-7 cells (Fig. 13B). TNF $\alpha$ increased the expression of $N F K B 1, N F K B 2, R E L$, and RELB, but not RELA in both MCF-7 and LCC9 cells (Fig. 16A-E). COUP-TFII overexpression inhibited the basal expression of REL and RELA in LCC9 cells (Fig. 16C and E). The TNF $\alpha$-induction of NFKB1, REL, and RELB was significantly reduced by COUP-TFII in LCC9 cells (Fig. 16A, C, and D). At the protein level, COUP-TFII overexpression decreased basal

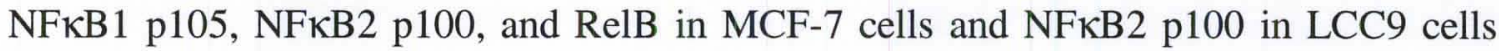
(Fig. 17). COUP-TFII inhibited the TNF $\alpha$-induced expression of NFкB2 p100, RelA, RelB, and c-Rel in MCF-7 cells and NFkB1 p105, RelA, and RelB in LCC9 cells (Fig. 17). Thus, COUP-TFII inhibits TNFo-induced increases in NFKB1 and RelB at both the mRNA and protein levels in LCC9. Differences between protein and mRNA results for NFKB subunit expression may have been due to the treatment conditions used for the experiment, as NFKB mRNA and protein levels may be altered at different timepoints. 

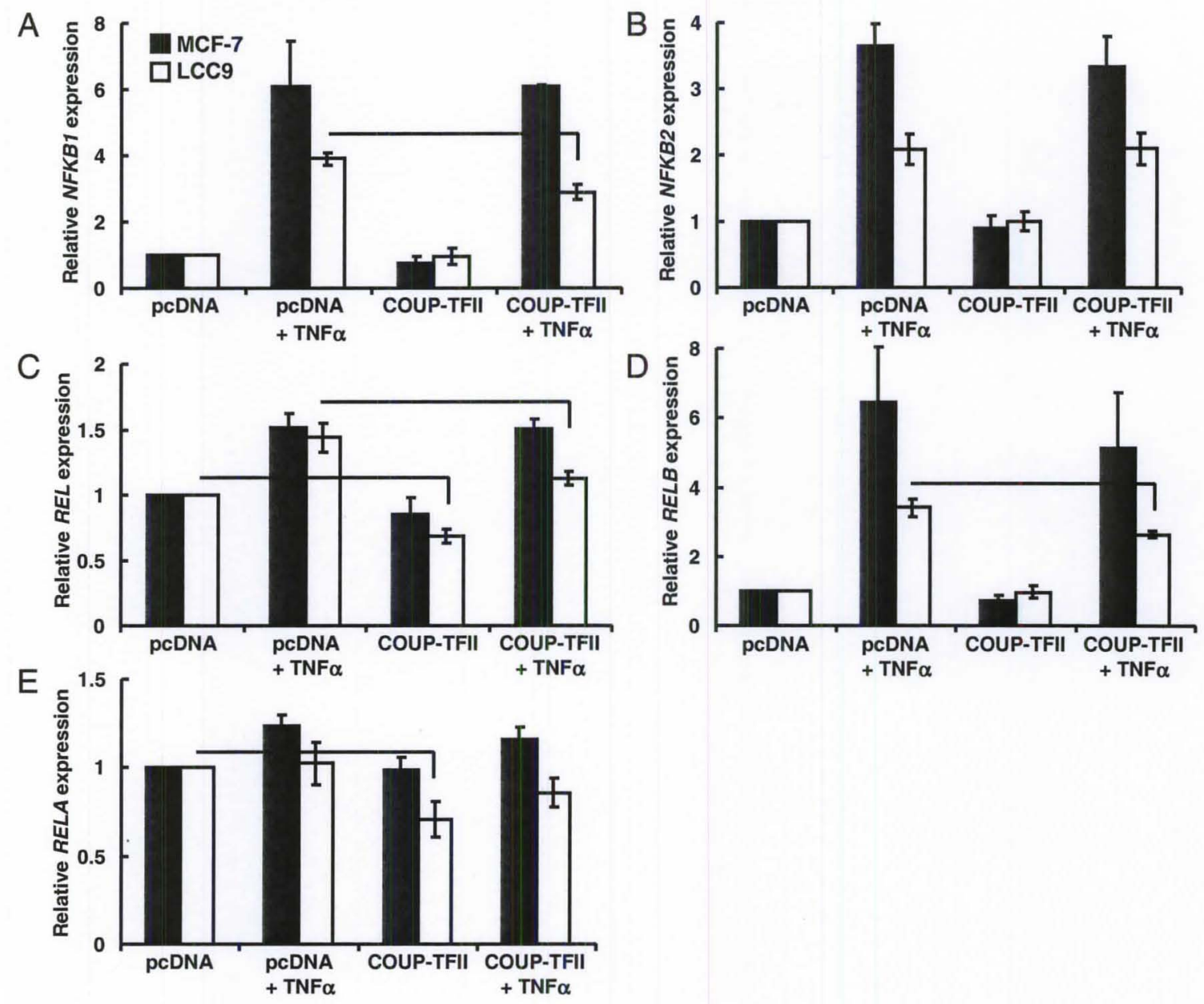

Figure 16. COUP-TFII inhibits mRNA expression of NFkB subunits in LCC9 cells. MCF-7 and LCC9 cells were transfected with pcDNA3.1 or pcDNA3.1-COUP-TFII for $48 \mathrm{~h}$ and treated with $10 \mathrm{ng} / \mathrm{ml}$ TNF $\alpha$ for $6 \mathrm{~h}$ before preparing RNA and cDNA. mRNA expression of NFKB subunits (A. NFKB1, B. NFKB2, C. REL, D. RELB, E. RELA) was analyzed by QRT-PCR. Bars indicate $\mathrm{p}<0.05$ between the indicated samples to indicate effect of COUP-TFII on basal or TNFo-induced gene expression in each cell line. 


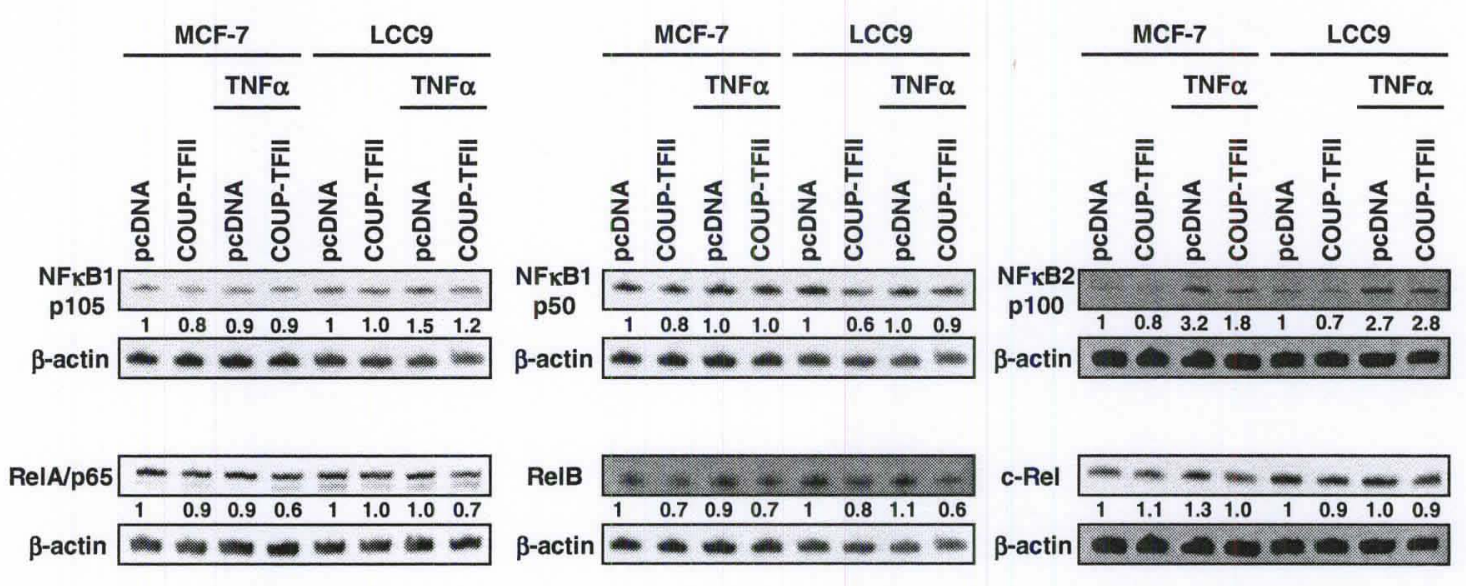

Figure 17. COUP-TFII inhibits protein expression of NFאB subunits.

MCF-7 and LCC9 cells were transfected with pcDNA3.1 or pcDNA3.1-COUP-TFII for $48 \mathrm{~h}$ and treated with $10 \mathrm{ng} / \mathrm{ml} \mathrm{TNF} \alpha$ for $6 \mathrm{~h}$ before preparing whole cell extracts.

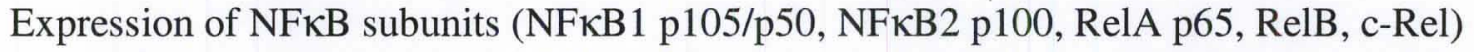
was analyzed by western blot with specific antibodies and compared to $\beta$-actin control. Note: NFkB2 p52 was not detectable. 
COUP-TFII and NFKB expression are inversely correlated in breast cancer patient samples

To examine the relationship between COUP-TFII and NFKB in breast cancer patients, publically available microarray gene expression data from $298 \mathrm{ER} \alpha+$ patients treated with tamoxifen for five years was analyzed using the Spearman Rho ( $\rho S)$ statistic to determine if there was a correlation between COUP-TFII expression and the expression of NFKB subunits and target genes. COUP-TFII (NR2F2) expression was inversely correlated with $\mathrm{NF \kappa B}$ subunit genes $N F K B 2$, REL, RELA, and RELB and NFKB target genes ICAM1, IL6, and TNFAIP3 (Table 4). In contrast, COUP-TFII (NR2F2) was positively correlated with NFkB target gene $C C L 2$ (Table 4). 
Table 4. Correlation of COUP-TFII expression to NFKB target and subunit gene expression in ER $\alpha+$ breast cancer patients treated with tamoxifen.

Spearman correlations of gene expression between COUP-TFII (NR2F2) and select

NFkB-related genes obtained by analysis of microarray data (GSE17705) from 298

$\mathrm{ER} \alpha+$ breast cancer patients treated with tamoxifen for five years.

\begin{tabular}{l|l|c|c|c}
\hline Classification & Gene & $\begin{array}{c}\text { Spearman } \\
\text { Rho }\end{array}$ & p-value & $\begin{array}{c}\text { Correlation to } \\
\text { COUP-TFII }\end{array}$ \\
\hline NFKB target & ICAM1 & -0.35 & $\mathbf{0 . 0 0 0 0}$ & Inverse \\
& IL6 & -0.16 & 0.0063 & Inverse \\
& TNFAIP3 & -0.23 & $\mathbf{0 . 0 0 0 0}$ & Inverse \\
& CCL2 & $\mathbf{0 . 1 4}$ & $\mathbf{0 . 0 1 5 2}$ & Positive \\
\hline NFKB subunit & NFKB1 & 0.08 & 0.1638 & \\
& NFKB2 & -0.20 & 0.0004 & Inverse \\
& REL & -0.28 & 0.0000 & Inverse \\
& RELA & -0.34 & 0.0000 & Inverse \\
& RELB & -0.12 & $\mathbf{0 . 0 4 2 8}$ & Inverse
\end{tabular}


COUP-TFII decreases NF KB-DNA binding

COUP-TFII can repress gene transcription by direct DNA binding and by direct protein:protein interaction with other nuclear receptors and transcription factors $[108,226]$. To determine if COUP-TFII inhibits NFkB-DNA binding, NE from LCC9 endocrine-resistant cells treated with $\mathrm{TNF} \alpha$ to activate $\mathrm{NF \kappa B}$ was incubated with increasing concentrations of in vitro transcribed/translated COUP-TFII protein and a $\left[{ }^{32} \mathrm{P}\right] \mathrm{NF} \kappa \mathrm{B}$ RE for an EMSA. In the absence of NE (lane 1), the $\left[{ }^{32} \mathrm{P}\right] \mathrm{NF}$ KB RE did not form a retarded band; however, addition of NE from TNF $\alpha$-treated LCC9 cells formed a retarded band (Fig. 18A). The intensity of the NFkB-DNA complex was decreased after competition with 100-fold excess of unlabeled NFKB oligomer (Appendix I, Fig. S11). COUP-TFII alone did not bind to the $\left[{ }^{32} \mathrm{P}\right] \mathrm{NFKB}$ RE oligomer (Fig. 18A); however, the amount of NFKB retarded complex was first slightly increased and then decreased with the addition of COUP-TFII in increasing concentrations (Fig. 18A). This suggests that COUP-TFII directly inhibits NFKB-DNA binding in vitro. A similar effect was seen using NE from MCF-7 and LCC9 cells transfected with COUP-TFII. In both MCF-7 endocrine-sensitive cells and LCC9 endocrine-resistant cells, transfection with COUPTFII decreased the amount of $\left[{ }^{32} \mathrm{P}\right] \mathrm{NF} \kappa \mathrm{B}$ RE bound complex (Fig. 18B). No increase was seen in $\left[{ }^{32} \mathrm{P}\right] \mathrm{NF} \kappa \mathrm{B}$ RE-binding with TNF $\alpha$ treatment in either MCF-7 or LCC9 cells (Fig. 18B), although induction of NFKB activation with TNF $\alpha$ treatment was detected in LCC9 cells under identical treatment conditions (Fig 13A). These data suggest that COUP-TFII interacts with and decreases NFKB-DNA complex formation. 


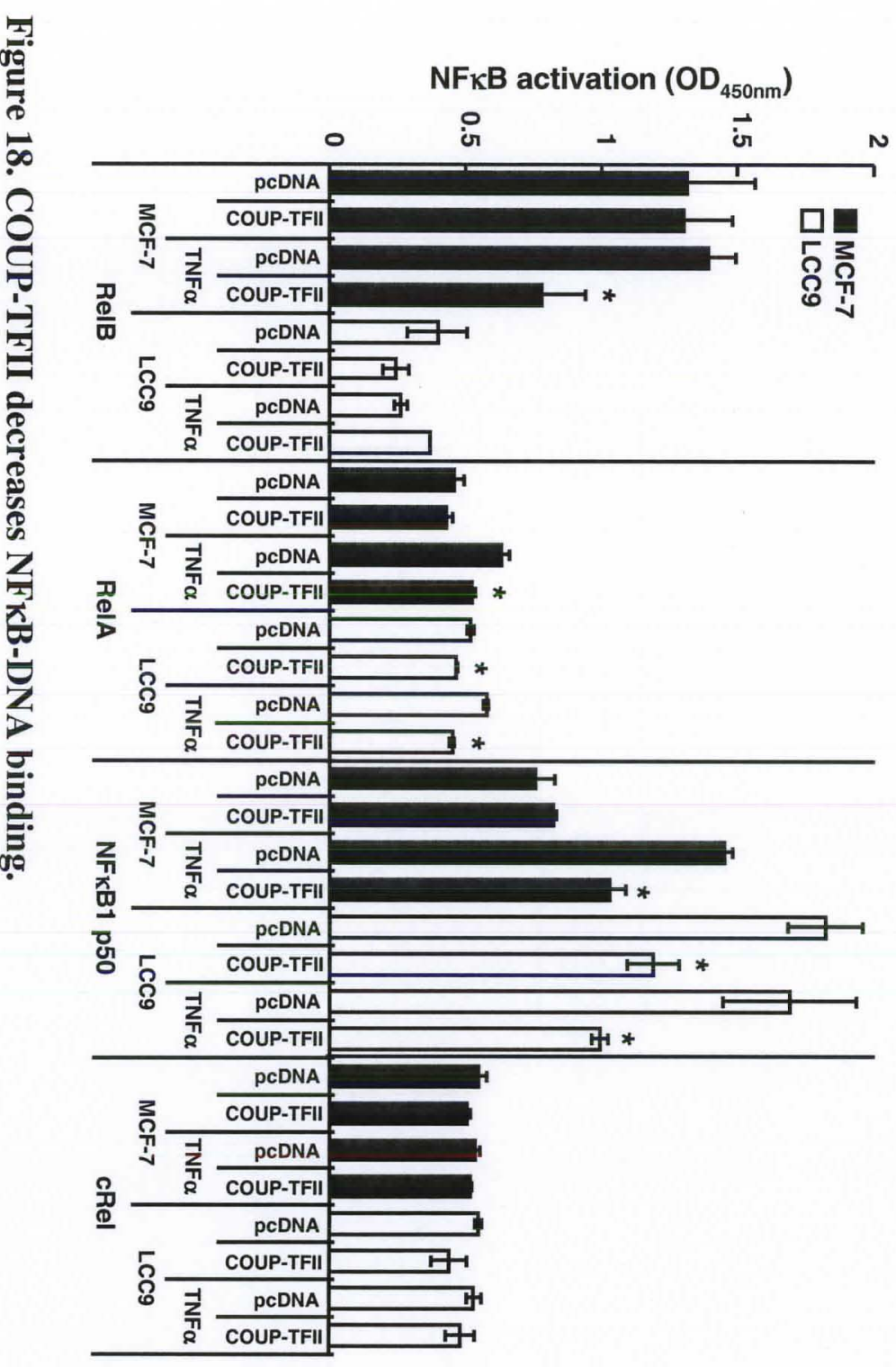

O

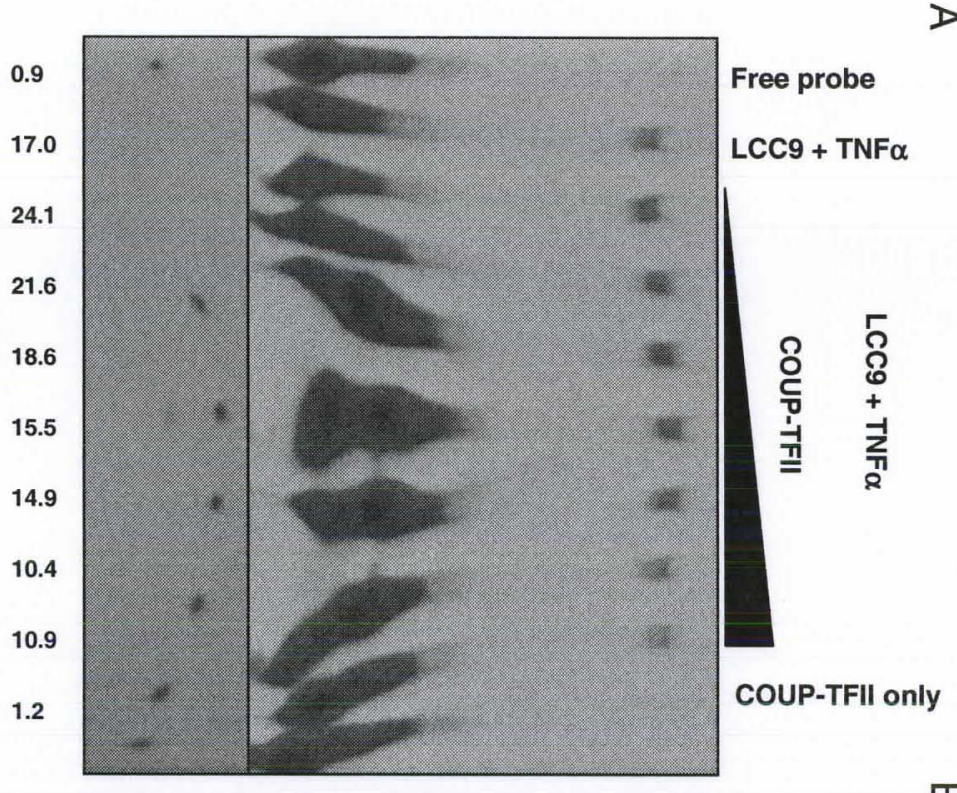

ए

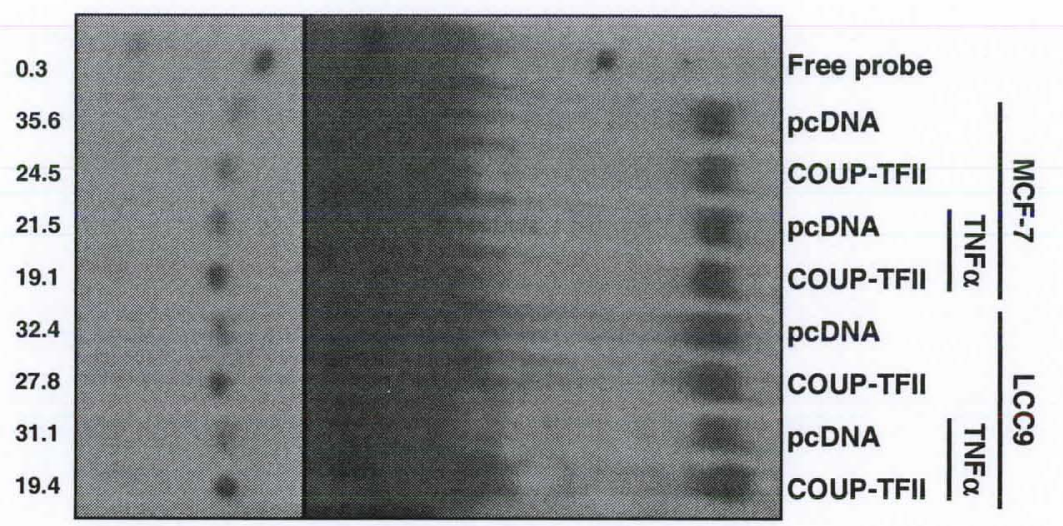


(A) NE (25 $\mu \mathrm{g}$ ) from TNF $\alpha$-treated (6 h) LCC9 cells was incubated alone or with increasing amounts of in vitro transcribed and translated COUP-TFII. [ $\left.{ }^{32} \mathrm{P}\right] \mathrm{NFKB}$ RE DNA probe was added for $1 \mathrm{~h}$ prior to EMSA. Values are relative bound $\left[{ }^{32} \mathrm{P}\right] \mathrm{NFKB}$ RE/ total $\left[{ }^{32} \mathrm{P}\right] \mathrm{NF} \kappa \mathrm{B}$ RE. (B) $25 \mu \mathrm{g}$ NE prepared from MCF-7 and LCC9 cells transfected with pcDNA3.1 or pcDNA3.1-COUP-TFII for $48 \mathrm{~h}$ and treated with TNF $\alpha$ for $6 \mathrm{~h}$ was incubated with $\left[{ }^{32} \mathrm{P}\right] \mathrm{NF} K \mathrm{~B}$ RE DNA probe for $1 \mathrm{~h}$ to perform EMSA. (A-B) Bottom panels contain lighter exposure of unbound $\left[{ }^{32} \mathrm{P}\right] \mathrm{NF} K \mathrm{~B}$ RE DNA probe. (C) TransAM $\mathrm{NFKB}$ family assays were performed to measure NFKB subunit activation using $15 \mu \mathrm{g}$ NE from MCF-7 and LCC9 cells transfected with pcDNA3.1 or pcDNA3.1-COUP-TFII for $48 \mathrm{~h}$ and treated with TNF $\alpha$ for $6 \mathrm{~h}$. Values are the average of two determinations \pm SEM. * Significantly different $\mathrm{p}<0.05$ from pcDNA-transfected cells. 
COUP-TFII inhibits RelB, RelA, and p50 NF $\kappa B$ activation

To examine the effect of COUP-TFII on specific NFKB subunit interaction with the NFKB RE, TransAM NFKB family assays were performed (Fig. 18C). RelB activity was higher in MCF-7 cells, while NFkB1 p50 activity was greater in LCC9. RelA and cRel activation were not statistically different between the two cell lines. NFKB2 p52 activation was low in MCF-7 or LCC9 (data not shown), consistent with the lack of p52 protein expression seen in western blots (Fig. 17). RelB activation was significantly decreased by COUP-TFII-transfection in TNF $\alpha$-treated MCF-7 cells. Activation of RelA and p50 was inhibited by COUP-TFII in both MCF-7 and LCC9 TNFo-treated cells as well as untreated LCC9 cells. COUP-TFII inhibited NFkB1/p50 more than RelA. These data suggest that COUP-TFII interferes with the binding of NFkB subunits RelB, RelA, and $\mathrm{p} 50$ to the NFKB RE.

\section{COUP-TFII interacts with NF $K B$ subunits}

As both COUP-TFII overexpression and in vitro incubation with COUP-TFII protein led to decrease in NFKB-DNA binding (Fig. 18A and B), reduced p50 and RelB activation (Fig. 18C), and inhibited target gene expression (Fig. 15A-D), coimmunoprecipitation was used to determine if COUP-TFII interacts with NFKB subunits to suppress their activity. Immunoprecipitation of COUP-TFII in MCF-7 cells revealed interaction between COUP-TFII and the NFkB subunits RelB and NFkB1 p105/p50 (Fig. 19A). 


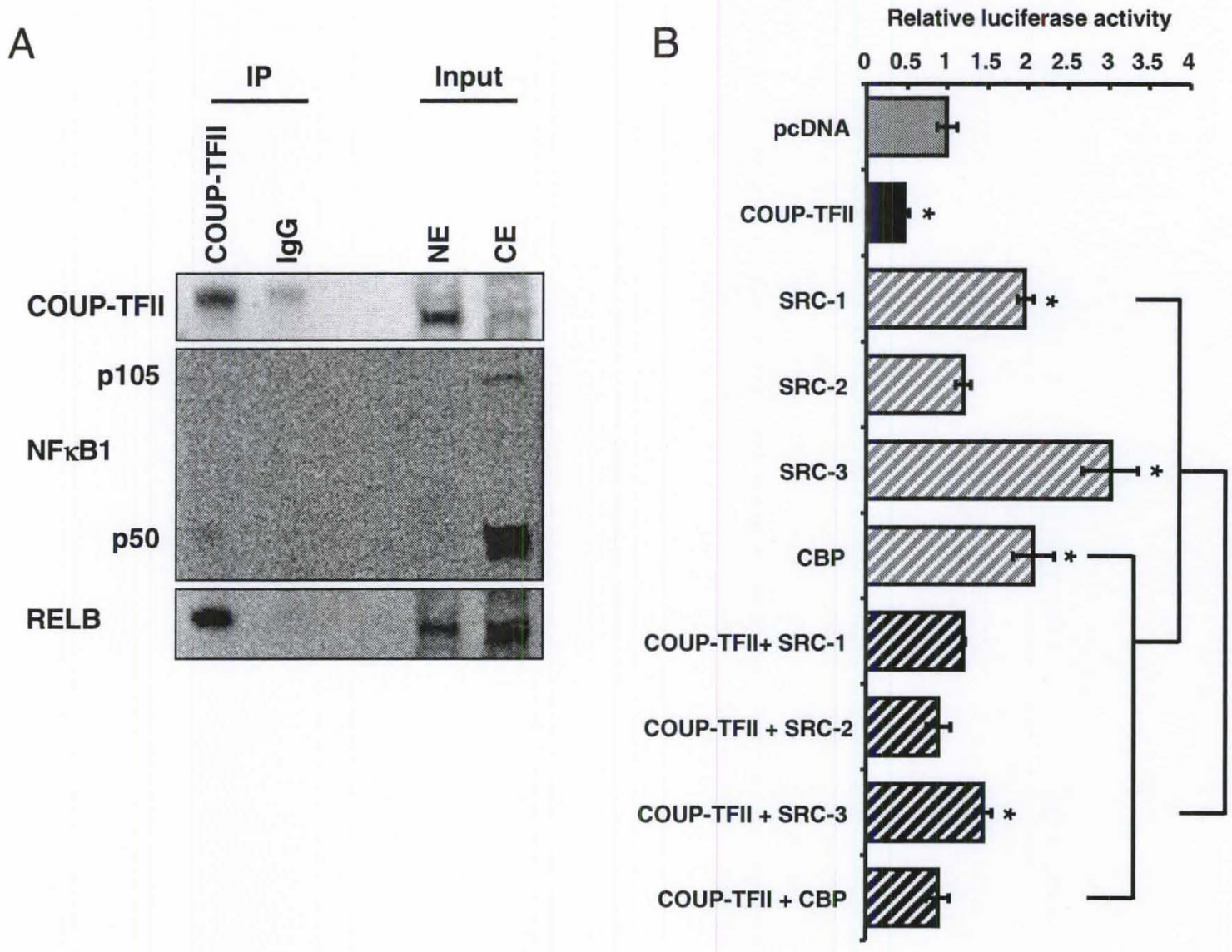

Figure 19. COUP-TFII interacts with NFKB subunits and inhibits coactivator function in NFKB activation.

(A) $500 \mu \mathrm{g} \mathrm{NE}$ from MCF-7 cells treated with TNF $\alpha$ for $6 \mathrm{~h}$ was immunoprecipitated (IP) using a COUP-TFII antibody and separated on a 10\% SDS PAGE gel prior to transfer to a PVDF membrane. Interaction with NFKB subunits NFKB1 p105/p50 and RelB was identified using specific antibodies on western blot. (B) LCC9 cells were transfected with a NFKB luciferase reporter and pcDNA3.1 or pcDNA3.1-COUP-TFII for $48 \mathrm{~h} \mathrm{+/}$ - plasmids for the coactivators SRC-1, SRC-2, SRC-3, and CBP. Cells were treated with $10 \mathrm{ng} / \mathrm{ml} \mathrm{TNF} \alpha$ for $6 \mathrm{~h}$ before performing dual luciferase assay. Firefly luciferase values are shown relative to protein concentration for a representative experiment of quadruplicate values \pm SEM. $*$ Significantly different $\mathrm{P}<0.05$ from pcDNA-transfected cells. Bars connect values which are significantly affected by COUP-TFII-transfection vs. coactivator alone. 
Inhibition of coactivator function by COUP-TFII reduces $N F K B$ transcriptional activity

COUP-TFII has also been reported to reported to inhibit the activity of androgen receptor (AR) by competing with coactivators for AR interaction [295]. SRC-1, SRC-2, SRC3, and CBP function as coactivators of NFKB-mediated transcription [296]. Each of these coactivators also have known interactions with COUP-TFII [226]. To determine if COUP-TFII represses NFKB signaling by interfering with coactivator function, LCC9 cells were cotransfected with a NFkB luciferase reporter with either pcDNA3.1 or pcDNA3.1-COUP-TFII plasmids (as in Fig. 13A), and plasmids for SRC-1, SRC-2, SRC-3, or CBP. The cells were treated with $10 \mathrm{ng} / \mathrm{ml} \mathrm{TNF} \alpha$ for 6 hours to activate NFkB. SRC-1, SRC-3, and CBP increased NFKB activity in LCC9 cells compared to parental vector pcDNA (Fig. 19B). SRC-2 transfection had no effect on NFkB activity. Cotransfection with COUP-TFII decreased the activation of NFkB activity by the coactivators SRC-1, SRC-3, and CBP (Fig. 19B), implicating COUP-TFII inhibition of coactivator function, e.g., competing with NFKB for coactivator interaction, as part of the mechanism of NFKB suppression.

\section{Endocrine-resistant cells are sensitive to $N F \kappa B$-inhibition}

Cell proliferation assays were performed to determine phenotypic effects of the both the activation of NFKB pathway in endocrine-resistant cells and the suppression of NFאB activity by COUP-TFII. MCF-7 and LCC9 cells overexpressing COUP-TFII were treated with combinations of TNF $\alpha$ to activate NFKB, 4-OHT to assess endocrinesensitivity, and BMS-345541, an NFkB inhibitor that functions by inhibiting IאB kinase 
and subsequent downstream NFKB activation [297]. BMS-345541 treatment was performed with a range of concentrations $(0.01-5 \mu \mathrm{M})$ determined to be effective in inhibiting NFאB activity in serum starved MCF-7 and LCC9 cells (Appendix I, Fig. S12). The proliferation of MCF-7 cells was largely unaffected by treatment with BMS-345541 (Fig. 20A). MCF-7 cells displayed reduced viability with 4-OHT or combined transfection with COUP-TFII and 4-OHT treatment (Fig. 20A), as previously reported [116] and in agreement with MCF-7's phenotype as endocrine-sensitive breast cancer cells. Treatment with TNF $\alpha$ did not significantly affect the response of MCF-7 to the compounds tested (Fig. 20B).

When LCC9 cells were treated with increasing concentrations of the NFkB inhibitor BMS-345541, a marked reduction in cell proliferation was observed (Fig. 20C), reflecting LCC9's increased reliance on a functional NFKB pathway for cell growth as endocrine-resistant cells [87,88]. Reduced LCC9 cell viability was observed upon combined transfection with COUP-TFII and treatment with 4-OHT, as has been previously reported [116]. LCC9 cells transfected/treated with a combination of COUPTFII, 4-OHT, and BMS-345541 exhibited a reduction in the higher range of BMS345541 concentrations (Fig. 20C). Interestingly, when LCC9 cells were treated with TNF $\alpha$, 4-OHT increased cell proliferation (Fig. 20D). This finding could have important translational implications if tamoxifen therapy may cause similar effects in patients with high levels of inflammation and NFKB activation. This observed induction of LCC9 cell proliferation with TNF $\alpha$ and 4-OHT treatment was blocked by transfection with COUPTFII and/or treatment with higher concentrations of BMS-345541 (Fig. 20D). 

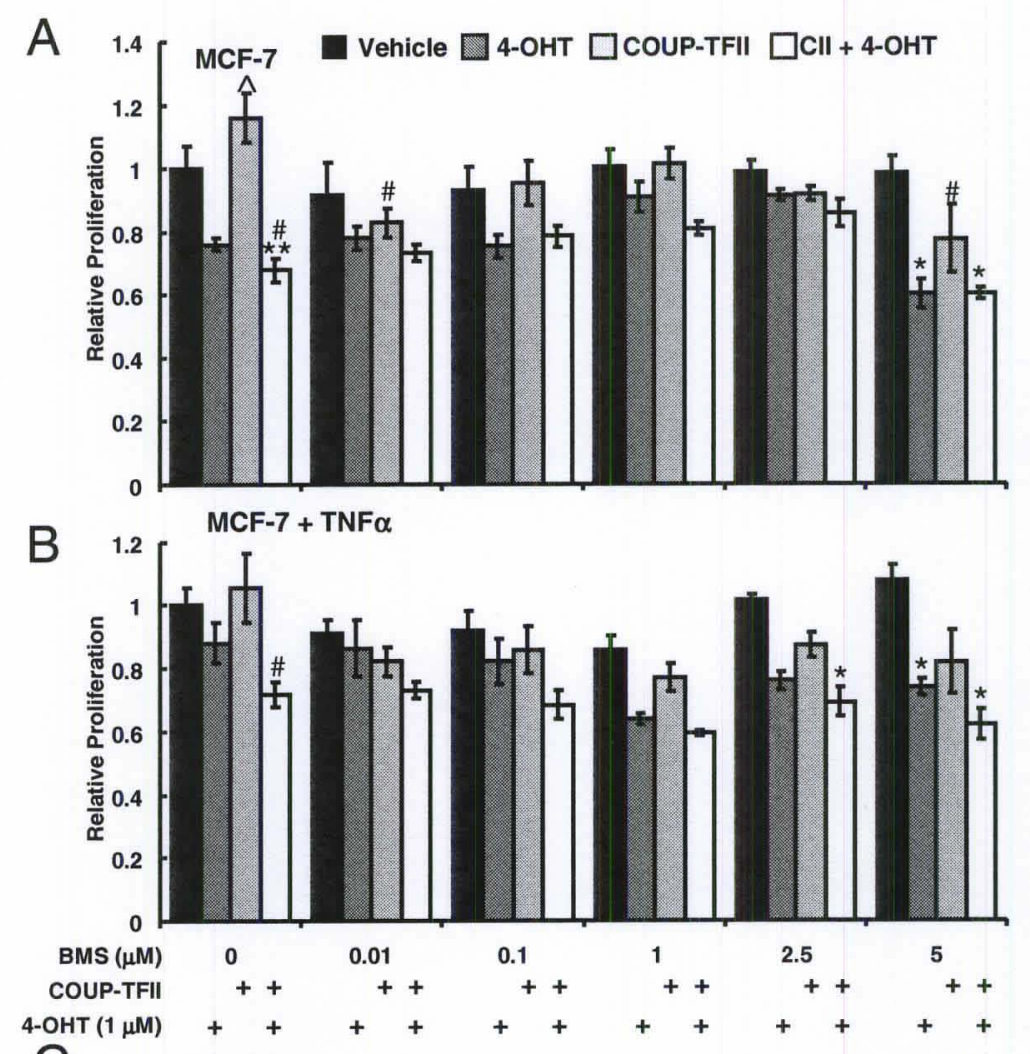

Figure 20.

Overexpression of COUP-TFII increases endocrine sensitivity in breast cancer cells. MCF-7 (A-B) and LCC9 (C-D) cells were serumstarved for $24 \mathrm{~h}$ before being transfected with pcDNA3.1 or pcDNA3.1-COUP-TFII for $24 \mathrm{~h}$. Cells were treated with concentrations of BMS345541 indicated, as well as $1 \mu \mathrm{M} 4-\mathrm{OHT}$ and $10 \mathrm{ng} / \mathrm{ml}$ TNF $\alpha$ for 5 days before performing MTT assay to assess changes in cell proliferation. $\mathrm{p}<0.05 * *$ vs. untreated, $\wedge$ vs. 4 OHT, \# vs. COUP-TFII, "vs. COUP-TFII + 4-

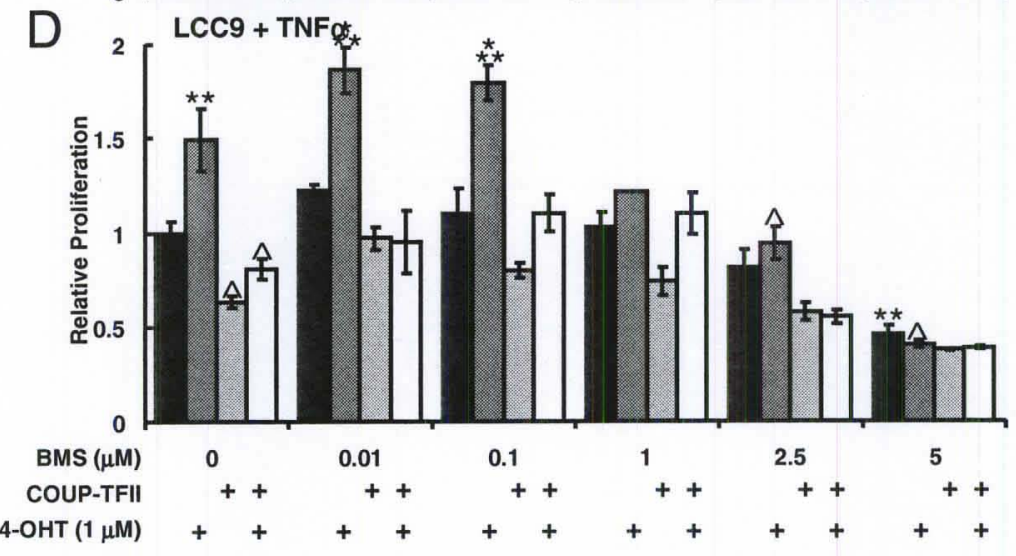
OHT, * vs. BMS-only at each concentration. 


\section{DISCUSSION}

The NFKB pathway is constitutively activated in many types of cancer, including

breast, gastrointestinal, genitourinary, hematological, and gynecological cancers, through a variety of mechanisms [86]. NFKB target genes have roles in oncogenic transformation, including apoptosis resistance [292,298], invasion [299], and epithelial to mesenchymal transition [300]. Here we demonstrate that COUP-TFII inhibits TNF $\alpha$ induced NFKB activity in LCC9 endocrine-resistant breast cancer cells. Consistent with previous reports that the NFKB pathway is activated during progression to breast cancer endocrine-resistance [87,88,91-93,301], LCC9 cells exhibited greater basal and TNF $\alpha$ induced NFKB activity than MCF-7 parental, endocrine-sensitive breast cancer cells. This inhibition led to downregulation of TNF $\alpha$-induced NFKB target gene expression of IL6, ICAM1, TNFAIP3, and CCL2, which had reduced basal and/or TNF $\alpha$-induced expression upon COUP-TFII overexpression. The inflammatory cytokine IL6 is a negative prognostic indicator for breast cancer patients [302], and may have a role in trastuzumab resistance [303]. Downregulation of the glycoprotein adhesion molecule ICAM1 at the mRNA and protein levels led to a strong suppression of human breast cell invasion [304]. The ubiquitin-editing enzyme A20/TNFAIP3 protects MCF-7 cells from TNF $\alpha$-induced apoptosis, and cells overexpressing TNFAIP3 are resistant to tamoxifen [292,298,305]. The chemokine ligand CCL2 may play a role in breast cancer development and progression, invasion and angiogenesis [299].

NFKB subunit expression is regulated by the NFKB pathway in a positive feedback loop. Specifically, RelA activates expression of RelB [306] and NFkB2 [307], while NFKB1/p50 expression is regulated by a combination of p50 and RelA [308]. 
Similarly, c-Rel is also capable of autoregulation [309]. Because COUP-TFII suppresses genes that are targets of the NFkB pathway, this would also lead to reduced expression of the NFKB subunits as observed. Consistent with our data showing higher NFKB subunit and target gene expression in LCC9 endocrine-resistant cells, NFKB subunits $N F K B 1$, NFKB2, RELA, and REL as well as the NFKB gene targets ICAM1, TNFAIP3, and CCL2 are also upregulated in inflammatory breast cancer patient samples [310]. A previous

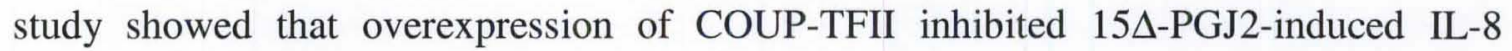
mRNA expression and promoter activity in U937 human myeloid cells, a result which the authors attributed to COUP-TFII competing with PPAR $\gamma$ to form a heterodimer with RXR or by forming a COUP-TFII/RXR heterodimer and competing with PPAR $\gamma / \mathrm{RXR}$ for DNA binding [311].

COUP-TFII was recently identified as one of four NRs that are significant predictors of metastasis-free survival in tamoxifen-treated breast cancers, independent of ER $\alpha$ expression [85]. We previously observed that COUP-TFII expression was reduced with histological grade [115] and this was confirmed in [85]. Here we report for the first time that COUP-TFII, i.e., $N R 2 F 2$ gene expression was inversely correlated with NFKB subunit genes $N F K B 2$, REL, RELA, and RELB and NFאB target genes ICAM1, IL6, and TNFAIP3 in microarray data from $298 \mathrm{ER} \alpha+$ breast tumors from patients treated with TAM for five years (Table 4). Although COUP-TFII inhibited NFkB target gene CCL2 in LCC9 cells (Fig. 15), COUP-TFII (NR2F2) was positively correlated with CCL2 in the breast tumors (Table 4), indicating potential differences between the patient population and cell line responses. This might be due to the effect of the breast tumor 
microenvironment in the patients, and expression of CCL2 by tumor-associated macrophages and other cells [299].

Mechanistically, I have demonstrated that COUP-TFII interacts with NFkB subunits RelB and NFkB1 p105/p50 and inhibits NFkB DNA-binding. COUP-TFII also inhibited the ability of coactivators SRC-1, SRC-3, and CBP to enhance NFKB activation. The effects of COUP-TFII on NFאB lead to inhibition of cell proliferation, with endocrine-resistant LCC9 cell proliferation showing the most reduction when treated with an NFKB inhibitor. Overall, these data indicate a general downregulation of NFKB target gene expression by COUP-TFII, which could have important phenotypic consequences on endocrine sensitivity. As NFKB is highly activated in endocrineresistant cells, blockage of downstream target gene expression by COUP-TFII may be a mechanism by which COUP-TFII enables breast cancer cells to maintain endocrine sensitivity. Natural product inhibitors of NFKB activity such as parthenolide [88], curcumin [312,313], and ellagic acid [314], as well as synthetic inhibitors [89,297] provide compounds for both experimental and future translational purposes. These data point to potential for the combination of $\mathrm{NF \kappa B}$ inhibitors with antiestrogen therapies, e.g. tamoxifen, to promote endocrine sensitivity, as well as the availability of COUP-TFII as a marker for response. 


\section{CHAPTER V - IDENTIFICATION OF COUP-TFII-INTERACTING PROTEINS IN TAMOXIFEN-TREATED BREAST CANCER CELLS}

\section{INTRODUCTION}

COUP-TFII is an orphan nuclear receptor that may play multiple roles in endocrine resistance. COUP-TFII expression is reduced in endocrine-resistant breast cancer cell lines, and overexpression of COUP-TFII restores endocrine sensitivity [116]. Part of the mechanism of maintenance of endocrine sensitivity by COUP-TFII involves its ability to suppress activation of NFkB pathway, as described in Chapter IV. As detailed in Chapter II, many pathways have been reported to be amplified in endocrine resistance and play important roles as both prognostic indicators and areas for potential therapeutic intervention, such as COUP-TFII. As outlined in previous chapters, the role of COUP-TFII in multiple pathways involved in cell differentiation, apoptotic resistance, migration, invasion, and others seems to be dependent on cellular context and interacting partners [226].

Mass spectrometry-based analyses to identify protein interactions can yield important information about disease states [315]. Protein interaction networks provide detailed pathways to enhance understanding of a protein in a particular cellular context [316]. Analyzing the roles of proteins within a particular protein complex or network produces valuable information about the specific activities of the proteins within the cell 
[317]. Proteomics is especially useful when studying drug resistance, resulting in additional angles from which to target a particular pathway [317]. As described in Chapter III, a previous study to identify COUP-TFII-interacting proteins in MCF-7 cells identified nucleolin as a coactivator for COUP-TFII-mediated transcription of retinoic acid receptor B2 (RARB2) [115].

The focus of the present study was to identify proteins that interact with COUPTFII in ethanol- versus tamoxifen-treated MCF-7 cells to define interacting proteins changed by treatment with tamoxifen. The goal of this study was to better understand the function of COUP-TFII in maintenance of breast cancer cellular response to tamoxifen treatment and how loss of COUP-TFII expression confers endocrine resistance to breast cancer cells. Heat shock protein 27 (HSP27) was identified amongst the proteins interacting with COUP-TFII more strongly in tamoxifen-treated cells. Given conflicting reports linking HSP27 to both increased and decreased drug resistance [318-325], HSP27 was selected for additional studies.

HSP27 interaction with COUP-TFII was confirmed in MCF-7 breast cancer cells by co-immunoprecipitation experiments. Regulation of HSP27 expression by estradiol and tamoxifen was investigated, as well as the expression and phosphorylation of HSP27 in both endocrine-resistant and trastuzumab-resistant breast cancer cells. Further studies are necessary to fully determine the relationship between HSP27 and COUP-TFII.

\section{MATERIALS AND METHODS}

\section{Chemicals and antibodies}


4-hydroxytamoxifen (4-OHT) and $17 \beta$-estradiol $\left(\mathrm{E}_{2}\right)$ were purchased from SigmaAldrich (St. Louis, MO). The following antibodies were purchased: polyclonal COUPTFII (Abcam, Cambridge, MA); monoclonal (mAB) anti-human COUP-TFII (R\&D systems, Minneapolis, MN; PP-H7147-00); mAB anti-FLAG M2 and $\beta$-actin (SigmaAldrich); mAB HSP27 (Santa Cruz, Santa Cruz, CA), polyclonal phospho-HSP27 (Ser82) and mAB HER2 (c-ErbB2/c-Neu, Millipore, Billerica, MA), HRP-conjugated secondary antibodies were from GE Healthcare (Piscataway, NJ).

\section{Cell culture}

MCF-7 breast cancer cells were purchased from ATCC and used at passage $<10$. Endocrine-resistant LCC2 [326], LCC9 [285], and LY2 [327] breast cancer cells were obtained from Robert Clarke (Georgetown University). MCF-7, LCC2, LCC9, and LY2 cells were maintained in improved MEM (IMEM) (Cellgro, Manassas, VA) supplemented with 5\% fetal bovine serum (FBS) (Atlanta Biologicals, Lawrenceville, $\mathrm{GA}$ ) and $1 \%$ penicillin/streptomycin (P/S, Cellgro). JIMT-1 trastuzumab-resistant breast cancer cells [328] were obtained from Gail Sonenshein (Tufts University) and maintained in DMEM (Cellgro) supplemented with $10 \%$ FBS and $1 \% \mathrm{P} / \mathrm{S}$. All cells were maintained in a $37^{\circ} \mathrm{C}$ incubator containing $5 \% \mathrm{CO}_{2}$.

\section{Immunoprecipitation of COUP-TFII-FLAG-interacting proteins}

MCF-7 cells were transfected with pIRES-COUP-TFII-FLAG for $24 \mathrm{~h}$ using FuGENE HD (Roche, Indianapolis, IN) according to manufacturer's instructions. Cells were 'serum-starved' using phenol red-free IMEM supplemented with 5\% dextran-coated 
charcoal-stripped serum (Atlanta Biologicals) for $24 \mathrm{~h}$ before being treated with $100 \mathrm{nM}$ 4-OHT or vehicle control EtOH for $2 \mathrm{~h}$. One mg of whole cell extract (WCE) prepared in lysis buffer (10\% glycerol, 50 mM HEPES pH 8.0, $100 \mathrm{mM} \mathrm{KCl,} 2$ mM EDTA, 0.1\% NP-40, 2 mM DTT, protease inhibitor cocktail (Roche), $10 \mathrm{mM} \mathrm{NaF}$, and $0.25 \mathrm{mM}$ $\mathrm{NaVO}_{4}$ ) [329] was incubated with EZview ${ }^{\mathrm{TM}}$ Red ANTI-FLAG® M2 Affinity gel (Sigma-Aldrich) for $4 \mathrm{~h}$ at $4^{\circ} \mathrm{C}$ with constant rotation. Washes and elution was performed in spin chromatography columns (BioRad, Hercules, CA). COUP-TFIIFLAG-interacting proteins were eluted by incubation with $100 \mu \mathrm{l} 6 \mathrm{M}$ urea $+100 \mathrm{mM}$ $\left(\mathrm{NH}_{4}\right) \mathrm{HCO}_{3}$ for $30 \mathrm{~min}$ with constant rotation. Three elution volumes were subsequently pooled for further analysis.

Identification of COUP-TFII-interacting proteins by 2D-LC-MS/MS

COUP-TFII-FLAG protein complexes were analyzed with a Thermo LTQ linear ion trap using a previously described 2D-LC-MS/MS approach [329]. Database searching was performed with tandem mass spectra extracted by ReadW and converted to mzXML format. Charge state deconvolution and deisotoping were not performed. All MS/MS samples were analyzed using SequestSorcerer (Sage-N, Milpitas, CA). Sequest was set up to search a FASTA-formatted human protein database (Human RefSeq, 2007). Searches were performed with a fragment ion mass tolerance of $1.00 \mathrm{Da}$ and a parent ion tolerance of 1.2 Da. Iodoacetamide derivative of cysteine was specified as a fixed modification. Oxidation of methionine was specified as a variable modification.

Scaffold (version Scaffold 3.0.00, Proteome Software Inc., Portland, OR) was used to validate MS/MS based peptide and protein identifications. Protein probabilities 
were assigned by the Protein Prophet algorithm [330]. Protein identifications were accepted if they could be established at greater than $95.0 \%$ probability. Proteins that contained similar peptides but could not be differentiated based on MS/MS analysis alone were grouped to satisfy the principles of parsimony.

Co-immunoprecipitation (co-IP) and immunoblotting

Nuclear and cytosolic proteins were prepared as in Chapter III [115]. $4 \mu \mathrm{g}$ COUP-TFII polyclonal antibody (Abcam) or rabbit IgG (Invitrogen, Carlsbad, CA) were added to $50 \mu \mathrm{l}$ Dynabeads Protein A (Invitrogen) in PBS (Cellgro) for $20 \mathrm{~min}$ at $4^{\circ} \mathrm{C}$. $400 \mu \mathrm{g}$ NE was added and incubated for $4 \mathrm{~h}$ at $4^{\circ} \mathrm{C}$ with rotation. Beads were washed $3 \mathrm{x}$ with PBS and bound proteins were eluted by incubation with Elution Buffer (Invitrogen). Proteins were separated by SDS-PAGE and analyzed by western blot $[115,116]$.

\section{Cell treatment}

Cells were 'serum-starved' for $48 \mathrm{~h}$ prior to treatment with ethanol (EtOH, vehicle control), $10 \mathrm{nM} \mathrm{E}_{2}$, or $100 \mathrm{nM} 4-\mathrm{OHT}$ for $6 \mathrm{~h}$, as indicated.

RNA Isolation, RT-PCR and Quantitative Real-Time-PCR (QRT-PCR)

RNA isolation, cDNA preparation, and QRT-PCR were performed as in [115]. QRT-PCR for HSP27 (HSPB1) and 18S, used Taqman Gene Expression Assays (Invitrogen). COUP-TFII (NR2F2) and GAPDH were measured using the SYBR green method as in [116]. All QRT-PCR was performed in the ViiA 7 Real-Time PCR system 
(Applied Biosystems, LifeTechnologies). Fold change was calculated from the $\Delta \Delta \mathrm{CT}$ values with the formula $2-\Delta \Delta \mathrm{CT}$ and data are relative to $\mathrm{EtOH}$-treated cells.

Statistical analysis

Values are reported as \pm SEM. Student's $t$ test was used for comparisons between control and treatment. $P$ values considered statistically significant are indicated.

\section{RESULTS}

Identification of COUP-TFII-associated proteins in ethanol-and tamoxifen-treated cells

To identify COUP-TFII-interacting proteins which are increased in their association with COUP-TFII either in EtOH (vehicle control) or 4-OHT treated MCF-7 cells, 2D-LC-MS/MS was performed following immunoprecipitation of COUP-TFIIFLAG and interacting proteins from EtOH and 4-OHT-treated MCF-7 cells. Efficiency of FLAG affinity purification is demonstrated in Figure 21. The majority of COUP-TFIIFLAG was bound by the FLAG affinity gel and not lost in flow through or washes (Figure 21A). COUP-TFII-FLAG was detected by western blot in both EtOH and 4OHT-treated cells and not in vector control-transfected cells (Figure 21B). From the mass spectrometry analysis, proteins were identified as interacting with COUP-TFII specifically in EtOH or 4-OHT-treated cells, as well as a group of proteins which were found in both EtOH and 4-OHT treated samples to varying degrees (Table 5). While three independent IP-mass spectrometry experiments were performed, COUP-TFII was not identified in the second experiment, and data from this experiment were not included in the summary of proteins identified as interacting with COUP-TFII. COUP-TFII- 
interacting proteins (from experiments 1 and 3) were subsequently categorically assigned as enriched in the ethanol or tamoxifen-treated groups, or equal/indistinguishable between the two (Table 5) based on their overall presence (number of spectra) in the replicate experiments. 


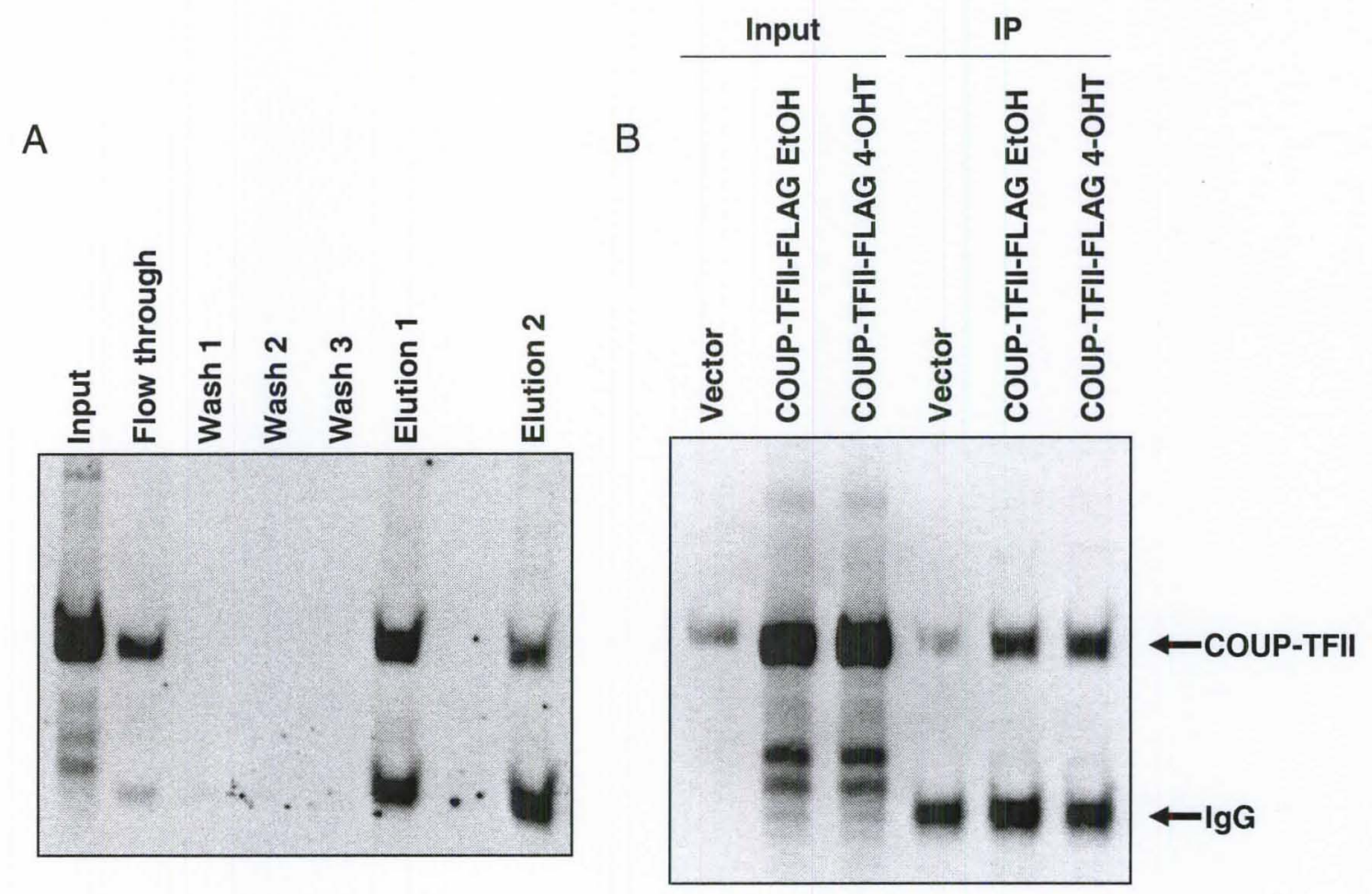

Figure 21. Efficiency of COUP-TFII-FLAG purification by FLAG affinity gel.

A, $20 \mu \mathrm{g}$ WCE from MCF-7 cells transfected with COUP-TFII-FLAG (input), $25 \mu \mathrm{l}$ flow through from incubation of WCE with FLAG affinity gel, $25 \mu \mathrm{l}$ IP washes, and $10 \mu \mathrm{l}$ of elutions 1 and 2 (10\% of elution volume) were analyzed by western blot to detect the presence of COUP-TFII-FLAG. B, $20 \mu \mathrm{g}$ WCE from empty vector pIRES or COUPTFII-FLAG-transfected cells treated with EtOH or 4-OHT (input) or $10 \mu \mathrm{l}$ of pooled elutions 1-3 were analyzed by western blot to detect the presence of COUP-TFII-FLAG before and after immunoprecipitation. 
Table 5. COUP-TFII-interacting proteins in ethanol- and tamoxifen-treated MCF-7 cells.

COUP-TFII-FLAG-transfected MCF-7 cells were treated with EtOH (Et) or $100 \mathrm{nM} 4-$ OHT (T) for $2 \mathrm{~h}$. $1 \mathrm{mg}$ WCE was incubated with anti-FLAG affinity gel. COUP-TFIIFLAG and interacting proteins were eluted with $6 \mathrm{M}$ urea and $100 \mathrm{mM}\left(\mathrm{NH}_{4}\right) \mathrm{HCO}_{3}$. 2DLC-MS/MS was used to identify proteins. The number of assigned spectra for identified proteins (Values) was normalized to number of spectra for COUP-TFII (bottom row in this Table) in each experiment. Qualitative assignment to ethanol, tamoxifen, and equalinteracting groups was made based on normalized values.

\begin{tabular}{|c|c|c|c|c|c|c|c|c|c|c|}
\hline & & & Expe & riment & & & Exp & erime & & \\
\hline & & Valu & & Norm & alized & Val & jes & Nor & alized & \\
\hline Identified Proteins & $\begin{array}{c}\text { MW } \\
(\mathrm{kDa})\end{array}$ & Et & $\mathrm{T}$ & Et & $\mathbf{T}$ & Et & $\mathrm{T}$ & Et & $\mathbf{T}$ & $\begin{array}{l}\text { Qualitative } \\
\text { enrichment }\end{array}$ \\
\hline myosin-9 (MYH9) & 227 & 102 & 24 & 3.00 & 0.67 & 10 & 4 & 3.33 & 4.00 & Ethanol \\
\hline $\begin{array}{l}\text { spectrin alpha } \\
\text { (SPTAN1) }\end{array}$ & 285 & 4 & 2 & 0.12 & 0.06 & 11 & 2 & 3.67 & 2.00 & Ethanol \\
\hline Calmodulin (CALM) & 17 & 10 & & 0.29 & & 7 & 2 & 2.33 & 2.00 & Ethanol \\
\hline (SPTBN1) & 275 & 4 & 0 & 0.12 & & 4 & & 1.33 & & Ethanol \\
\hline $\begin{array}{l}\text { tubulin alpha-1B chain } \\
\text { (TUBA1B) }\end{array}$ & 50 & 41 & 50 & 1.21 & 1.39 & 6 & 10 & 2.00 & 10.00 & Tamoxifen \\
\hline $\begin{array}{l}\text { RNA-binding protein } \\
\text { FUS (FUS) }\end{array}$ & 53 & 25 & 26 & 0.74 & 0.72 & 6 & & 2.00 & & Tamoxifen \\
\hline $\begin{array}{l}\text { serum albumin } \\
\text { preproprotein (ALB) } \\
\text { heterogeneous nuclear }\end{array}$ & 69 & 10 & 8 & 0.29 & 0.22 & 2 & 2 & 0.67 & 2.00 & Tamoxifen \\
\hline $\begin{array}{l}\text { ribonucleoprotein U } \\
\text { (HNRNPU) }\end{array}$ & 89 & 9 & 10 & 0.26 & 0.28 & 2 & 2 & 0.67 & 2.00 & Tamoxifen \\
\hline $\begin{array}{l}\text { keratin, type I } \\
\text { cytoskeletal } 10 \text { (KRT10) }\end{array}$ & 59 & 65 & 59 & 1.91 & 1,64 & 2 & 2 & 0.67 & 2.00 & Tamoxifen \\
\hline $\begin{array}{l}60 S \text { acidic ribosomal } \\
\text { protein P1 (RPLP1) } \\
\text { probable ATP. }\end{array}$ & 12 & 4 & 5 & 0.12 & 0.14 & 2 & 2 & 0.67 & 2.00 & Tamoxifen \\
\hline $\begin{array}{l}\text { dependent RNA } \\
\text { helicase DDX5 (DDX5) }\end{array}$ & 69 & 28 & 27 & 0.82 & 0.75 & 2 & 2 & 0.67 & 2.00 & Tamoxifen \\
\hline $\begin{array}{l}\text { homeobox prox } 1 \\
\text { (PROX1) }\end{array}$ & 28 & 14 & 16 & 0.41 & 0.44 & 2 & 2 & 0.67 & 2.00 & Tamoxifen \\
\hline $\begin{array}{l}\text { heat shock cognate } 71 \\
\text { kDa protein (HSPA8) }\end{array}$ & 71 & 48 & 53 & 1.41 & 1.47 & 2 & 4 & 0.67 & 4.00 & Tamoxifen \\
\hline $\begin{array}{l}\text { regulated protein } \\
\text { precursor (HSPA5) }\end{array}$ & 72 & 23 & 30 & 0.68 & 0.83 & 2 & 2 & 0.67 & 2.00 & Tamoxifen \\
\hline $\begin{array}{l}\text { heat shock protein beta- } \\
1 \text { (HSPB1) }\end{array}$ & 23 & 10 & 14 & 0.29 & 0.39 & & 2 & & 2.00 & Tamoxifen \\
\hline $\begin{array}{l}\text { subunit alpha-1 } \\
\text { (CAPZA1) }\end{array}$ & 33 & 6 & 3 & 0.18 & 0.08 & & 2 & & 2.00 & Tamoxifen \\
\hline histone $\mathrm{H} 4$ (HIST1H4) & 11 & 34 & 27 & 1.00 & 0.75 & 8 & 4 & 2.67 & 4.00 & Equal \\
\hline filamin-A (FLNA) & 280 & 35 & 40 & 1.03 & 1.11 & 8 & 2 & 2.67 & 2.00 & Equal \\
\hline myosin-14 (MYH14) & 228 & 7 & & 0.21 & & 4 & 2 & 1.33 & 2.00 & Equal \\
\hline
\end{tabular}




\begin{tabular}{|c|c|c|c|c|c|c|c|c|c|c|}
\hline $\begin{array}{l}\text { keratin, type II } \\
\text { cytoskeletal } 8 \text { (KRT8) }\end{array}$ & 54 & 77 & 93 & 2.26 & 2.58 & 5 & 2 & 1.67 & 2.00 & Equal \\
\hline myosin-lb (MYO1B) & 125 & 8 & 1 & 0.24 & 0.03 & 4 & 2 & 1.33 & 2.00 & Equal \\
\hline $\begin{array}{l}\text { keratin, type I } \\
\text { cytoskeletal } 9 \text { (KRT9) } \\
\text { 2,4-dienoyl-CoA }\end{array}$ & 62 & 63 & 38 & 1.85 & 1.06 & 2 & 2 & 0.67 & 2.00 & Equal \\
\hline $\begin{array}{l}\text { reductase, mitochondrial } \\
\text { precursor (DECR1) }\end{array}$ & 36 & 6 & 6 & 0.18 & 0.17 & 3 & 1 & 1.00 & 1.00 & Equal \\
\hline $\begin{array}{l}\text { keratin, type II } \\
\text { cytoskeletal } 1 \text { (KRT1) }\end{array}$ & 66 & 53 & 47 & 1.56 & 1.31 & 1 & & 0.33 & & Equal \\
\hline myosin-lc (MYO1C) & 122 & 4 & & 0.12 & & 3 & & 1.00 & & Equal \\
\hline $\begin{array}{l}\text { complementing protein } 6 \\
\text { (XRCC6) } \\
\text { plasminogen activator }\end{array}$ & 70 & 59 & 56 & 1.74 & 1.56 & 3 & & 1.00 & & Equal \\
\hline $\begin{array}{l}\text { Inhibitor } 1 \text { RINA-binding } \\
\text { protein (SERBP1) }\end{array}$ & 45 & 21 & 21 & 0.62 & 0.58 & & 1 & & 1.00 & Equal \\
\hline $\begin{array}{l}\text { COUP transcription } \\
\text { factor } 2 \text { (NR2F2) }\end{array}$ & 46 & 34 & 36 & 1.00 & 1.00 & 3 & 1 & 1.00 & 1.00 & \\
\hline
\end{tabular}


Functional Annotation Clustering of COUP-TFII-interacting proteins

The Database for Annotation, Visualization, and Integrated Discovery (DAVID, version 6.7) [331,332] was used to identify functionally related groups amongst the identified COUP-TFII-interacting proteins in the ethanol, tamoxifen, and equally enriched groups (Table 6). This bioinformatic analysis allows for detection of biological themes amongst groups of proteins to identify pathways that may be relevant in a particular state [331,332]. Gene lists compiled from Table 5 were entered into the DAVID database and functional annotation clustering was performed using high stringency for analysis. Repeated terms were removed from the resulting list (Table 6).

Common themes amongst the groups were calmodulin-binding (ethanol and equal) and RNA splicing/alternative splicing (ethanol and tamoxifen) (Table 6), suggesting these may have importance to COUP-TFII. As calmodulin was identified amongst the COUP-TFII-interacting proteins (Table 6), this may indicate that this interaction has significance. A role for COUP-TFII in RNA splicing has not yet been reported, and this may also be a new area for study given potential interactions with DEAD box protein 5 (DDX5), RNA-binding protein FUS (FUS), and heterogeneous nuclear ribonucleoprotein $\mathrm{U}$ (HNRNPU) with tamoxifen treatment (Table 5 and 6). 
Table 6. Functional Annotation Clustering of COUP-TFII-interacting proteins. Proteins identified as enriched in the EtOH or tamoxifen-treated samples or proteins identified as interacting with COUP-TFII relatively the same with either treatment were entered into the DAVID database to identify biological relationships between the proteins.

\begin{tabular}{lcccc}
\hline Term & Count & p-value & $\begin{array}{c}\text { Fold } \\
\text { Enrichment }\end{array}$ & Bonferroni \\
\hline & & & & \\
ETHANOL & & & & \\
Cluster 1: MYH9, SPTAN1, SPTBN1 & 3 & $4.3 \mathrm{E}-05$ & 195.64 & 0.003 \\
Cortical cytoskeleton & 3 & $1.2 \mathrm{E}-04$ & 120.22 & 0.004 \\
Calmodulin-binding & 3 & $1.2 \mathrm{E}-04$ & 115.50 & 0.008 \\
Cell cortex & 3 & $4.9 \mathrm{E}-04$ & 58.41 & 0.018 \\
Actin-binding & 3 & $7.9 \mathrm{E}-02$ & 4.35 & 0.996 \\
Plasma membrane part & 3 & $3.4 \mathrm{E}-01$ & 1.93 & 1.000 \\
Alternative splicing & & & & \\
& & & & \\
Cluster 2: MYH9, SPTAN1, CALM, SPTBN1 & & & & \\
Cytoskeletal protein binding & 4 & $5.8 \mathrm{E}-05$ & 25.76 & 0.002 \\
Acetylation & 4 & $2.6 \mathrm{E}-03$ & 7.30 & 0.091 \\
Non-membrane-bounded organelle & 4 & $8.4 \mathrm{E}-03$ & 4.92 & 0.435 \\
Plasma membrane & 4 & $2.6 \mathrm{E}-02$ & 3.38 & 0.831 \\
Phosphoprotein & 4 & $5.4 \mathrm{E}-02$ & 2.65 & 0.871 \\
Polymorphism & 4 & $2.2 \mathrm{E}-01$ & 1.67 & 1.000 \\
& & & & \\
\hline
\end{tabular}

TAMOXIFEN

Cluster 1: DDX5, FUS, HNRNPU

RNA splicing

$\begin{array}{lll}0.018 & 12.99 & 0.918\end{array}$

Cluster 2: DDX5, HSPA8, HNRNPU, TUBA1B, HSPA5

Nucleotide-binding

$\begin{array}{llll}5 & 0.012 & 4.75 & 0.568\end{array}$

Cluster 3: ALB, HSPB1, HSPA5

Negative regulation of apoptosis

$\begin{array}{llll}3 & 0.027 & 10.42 & 0.978\end{array}$

Cluster 4: ALB, HSPA8, HSPA5

Cytoplasmic membrane-bounded vesicle

$\begin{array}{lll}3 & 0.079 & 5.81\end{array}$

0.997

Cluster 5: DDX5, HSPA8, HNRNPU, HSPA5

ATP-binding

$\begin{array}{lll}4 & 0.036 & 4.84\end{array}$

0.923

Cluster 6: ALB, FUS, HSPA5 
EQUAL

Cluster 1: MY01B, MYO1C, MYO14

Myosin

Calmodulin-binding region

$\begin{array}{llll}3 & 3.1 \mathrm{E}-04 & 102.86 & 0.019 \\ 3 & 9.5 \mathrm{E}-04 & 57.44 & 0.026\end{array}$

Cluster 2: MYO1B, MY01C, MYO14, FLNA

Actin-binding

$\begin{array}{llll}4 & 2.3 \mathrm{E}-04 & 28.32 & 0.015\end{array}$

Cytoskeletal protein binding

$\begin{array}{llll}4 & 5.7 \mathrm{E}-03 & 9.37 & 0.310\end{array}$

Cluster 3: KRT1, KRT8, KRT9

Intermediate filament

Cytoskeletal keratin

Structural molecule activity

7.2E-04

67.26

0.044

$3 \quad 1.8 \mathrm{E}-03$

40.42

0.013

$3 \quad 8.3 \mathrm{E}-02$

5.58

0.996

Cluster 4: MYO1B, MYO1C, MY014, XRCC6

ATP-binding

Nucleotide-binding

$\begin{array}{llll}4 & 2.7 \mathrm{E}-02 & 5.27 & 0.824 \\ 4 & 5.1 \mathrm{E}-02 & 4.15 & 0.962\end{array}$

Cluster 5: XRCC6, HIST1H4, MYO1C

Nucleoplasm

$3 \quad 1.5 \mathrm{E}-01$

3.95

1.000

Intracellular organelle lumen

$34.2 \mathrm{E}-01$

1.96

1.000 
Endogenous nuclear interaction of COUP-TFII with HSP27

Heat shock protein beta-1 (HSPB1), also known as heat shock protein 27 (HSP27), is a member of the small heat shock protein family of chaperones that are involved in maintenance of protein stability and prevention of abnormal protein folding or aggregation [333,334]. HSP27 is increased expression in breast carcinoma and other cancers and can inhibit caspase-dependent apoptosis [335-337]. HSP27 expression has been reported to be increased by chemotherapy in breast cancer patients and to be correlated with reduced disease free survival [318]. HSP27 is known to interact with $\mathrm{ER} \beta$ [338], ER $\alpha$, progesterone receptor (PR), and androgen receptor (AR) [339]. Based on these reports, HSP27 was selected from the list of proteins identified to interact with COUP-TFII, and enriched in cells treated with 4-OHT, for further investigation.

Endogenous COUP-TFII-HSP27 interaction was examined in MCF-7 cells. HSP27 is abundantly present in both the NE and CE of MCF-7 cells, while COUP-TFII is only present in the NE (Fig. 22A). HSP27 was detected via western blot following IP of NE with a polyclonal COUP-TFII antibody (Fig. 22A). No HSP27 was present in the negative control IgG or antibody-only lanes (Fig. 22A). Interaction of COUP-TFII with calmodulin, another protein identified by mass spectrometry to interact with COUP-TFI, although enriched when cells were treated with $\mathrm{EtOH}$ (Table 5), was also verified through IP (Fig. 22A).

To examine whether COUP-TFII-HSP27 interaction was altered following treatment with 4-OHT, a similar IP was performed using NE prepared from MCF-7 cells treated with EtOH (vehicle control) or 4-OHT for $2 \mathrm{~h}$ (Fig. 22B). No significant change in COUP-TFII-HSP27 interaction was observed following treatment with 4-OHT as 
compared to EtOH (Fig. 22B). COUP-TFII and HSP27 expression and subcellular localization were also not affected by treatment (Fig. 22B). COUP-TFII interaction with calmodulin was also verified via IP in MCF-7 cells (Fig. 22A), although follow-up experiments to elucidate the function of this interaction have not yet been performed. 

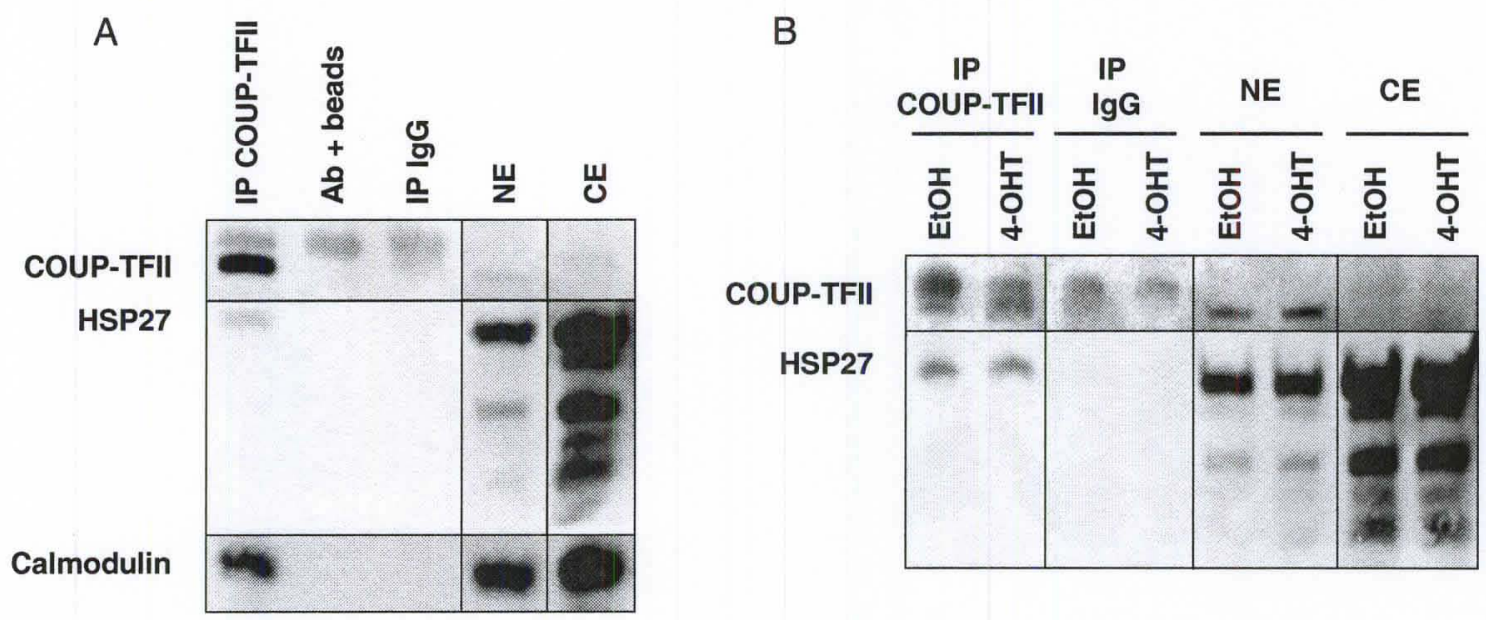

Figure 22. Endogenous nuclear interaction of COUP-TFII with HSP27 and calmodulin in MCF-7 cells.

$400 \mu \mathrm{g}$ NE from MCF-7 cells was immunoprecipitated with polyclonal COUP-TFII antibody or negative control rabbit IgG (negative control) and analyzed by western blot for COUP-TFII and interacting proteins identified via mass spectrometry. $40 \mu \mathrm{g} \mathrm{NE}$ or CE was used as input control. A, COUP-TFII interacts with HSP27 and calmodulin in MCF-7 cells. B, COUP-TFII interacts with HSP27 in EtOH and 4-OHT-treated MCF-7 cells. 
Regulation of HSP27 by estradiol and tamoxifen in endocrine-resistant breast cancer cells

The promoter region of HSP27 contains a Sp1 and half ERE site reported to be responsible for the upregulation of HSP27 expression by $E_{2}$ through a Sp1/ER complex [340]. To examine both the relative expression of HSP27 in breast cancer cell lines with increasing endocrine resistance, as well as the effect of $\mathrm{E}_{2}$ and 4-OHT treatment on HSP27 expression, MCF-7, LCC1, LCC2, LCC9, and LY2 cells were treated with $10 \mathrm{nM}$ $\mathrm{E}_{2}, 100 \mathrm{nM}$ 4-OHT, or EtOH (vehicle control) for $6 \mathrm{~h}$. Increased HSP27 expression was observed in LCC1 estrogen-independent but tamoxifen-sensitive cells [326] as compared to MCF-7 estrogen-dependent, tamoxifen-sensitive cells. LCC2 cells, which are both estrogen-independent and tamoxifen-resistant [326], had reduced HSP27 expression compared to MCF-7. LCC9, estrogen-independent, tamoxifen and fulvestrant-resistant cells [285], displayed a further reduction in HSP27 expression. LY2, a breast cancer cell line that is resistant to tamoxifen, fulvestrant, and LY117018 (a raloxifene analog) [327] and has undergone epithelial to mesenchymal transition, had undetectable expression of HSP27 by QRT-PCR.

Previous reports have indicated regulation of HSP27 by estrogen signaling [340]; however, this was not confirmed in these experiments. $E_{2}$ treatment had no statistically significant effect on HSP27 expression in any of the cell lines examined (Fig. 23), reflecting potential differences the breast cancer cell lines used by investigators, the methods of detection, altered endocrine responses of the tamoxifen-resistant cell lines, etc. 4-OHT treatment in LCC1 cells lead to a slight increase in HSP27 expression (Fig. 23), while no effect was observed in other cell lines. Increased HSP27 expression in 
response to 4-OHT treatment would be consistent with reports that cellular stress and drug treatment induce HSP27 expression [336,341].

HSP27 and COUP-TFII expression is reduced in endocrine-resistant and trastuzumabresistant breast cancer cells

To follow up on the observed differences in steady state mRNA expression of HSPB1 (Fig. 23), HSP27 expression was also analyzed by western blot of WCE from endocrine-sensitive MCF-7 cells and endocrine-resistant LCC2, LCC9, and LY2 cells. As seen at the mRNA level (Fig. 23), a reduction in HSP27 expression was prominent in LY2 cells (Fig. 24A). LCC2 and LCC9 cells displayed HSP27 expression approximately equivalent to MCF-7 cells. Interestingly, the lower molecular weight band of HSP27 seen in MCF-7, LCC2, and LCC9 was absent in LY2 cells (Fig. 24A). HSP27 expression was also examined in a trastuzumab-resistant breast cancer cell line, JIMT-1 (Fig. 24B). Trastuzumab (Herceptin) is a monoclonal antibody used for the treatment of HER2+ breast cancer patients [12]. JIMT-1 cells display clinical resistance to trastuzumab, as they were derived from a pleural effusion of a patient whose disease had progressed to trastuzumab-resistance [328]. Similar to LY2 cells, HSP27 expression was reduced in JIMT-1 cells, and the lower band seen in MCF-7 cells was not present (Fig. 24B).

Phosphorylation of HSP27 by MAPK at serine 15, 78, and 82 has also been shown to be important to its cellular function and localization [319,325]. Cell stress, such as heat shock, chemotherapy, or cytokine exposure, can induce phosphorylation of HSP27 [337]. Phosphorylation of HSP27 at Ser82 was also examined in the same cell 
lines, and the pattern of p-Ser82-HSP27 was found to mirror the expression of HSP27 in all 5 cell lines examined (Fig. 24A and B).

To investigate a possible correlation between HSP27 and COUP-TFII expression, COUP-TFII expression was also analyzed by western blot. As previously reported [116], COUP-TFII expression is reduced in the endocrine-resistant LCC2, LCC9, and LY2 cells as compared to MCF-7 (Fig. 24A). COUP-TFII expression was also much lower in JIMT-1 cells compared to MCF-7 endocrine-sensitive cells, suggesting a potential role for COUP-TFII in trastuzumab-resistance (Fig. 24B).

\section{COUP-TFII/HSP27 and HER2 expression are inversely correlated}

We have previously reported an inverse correlation between COUP-TFII and HER2 expression in tamoxifen-treated patient tumors via immunohistochemistry [115]. To determine if COUP-TFII and HSP27 expression are correlated to HER2 expression in the endocrine-resistant and trastuzumab-resistant cell lines, a western blot was performed using a HER2 antibody. HER2 expression was undetectable in MCF-7 and LCC2 cells (Fig. 24A). Very low expression of HER2 was detected in LCC9 cells, while LY2 cells had the greatest HER2 expression of the endocrine-resistant cell lines (Fig. 24A). JIMT1 cells are trastuzumab-resistant and, as part of their identified phenotype, have been reported to have increased expression of HER2 [328]. JIMT-1 cells had the highest HER2 expression detected in any of the cell lines examined (Fig. 24B). These data suggest a trend towards an inverse pattern of expression between COUP-TFII/HSP27 and HER2 in the cell lines examined herein. 


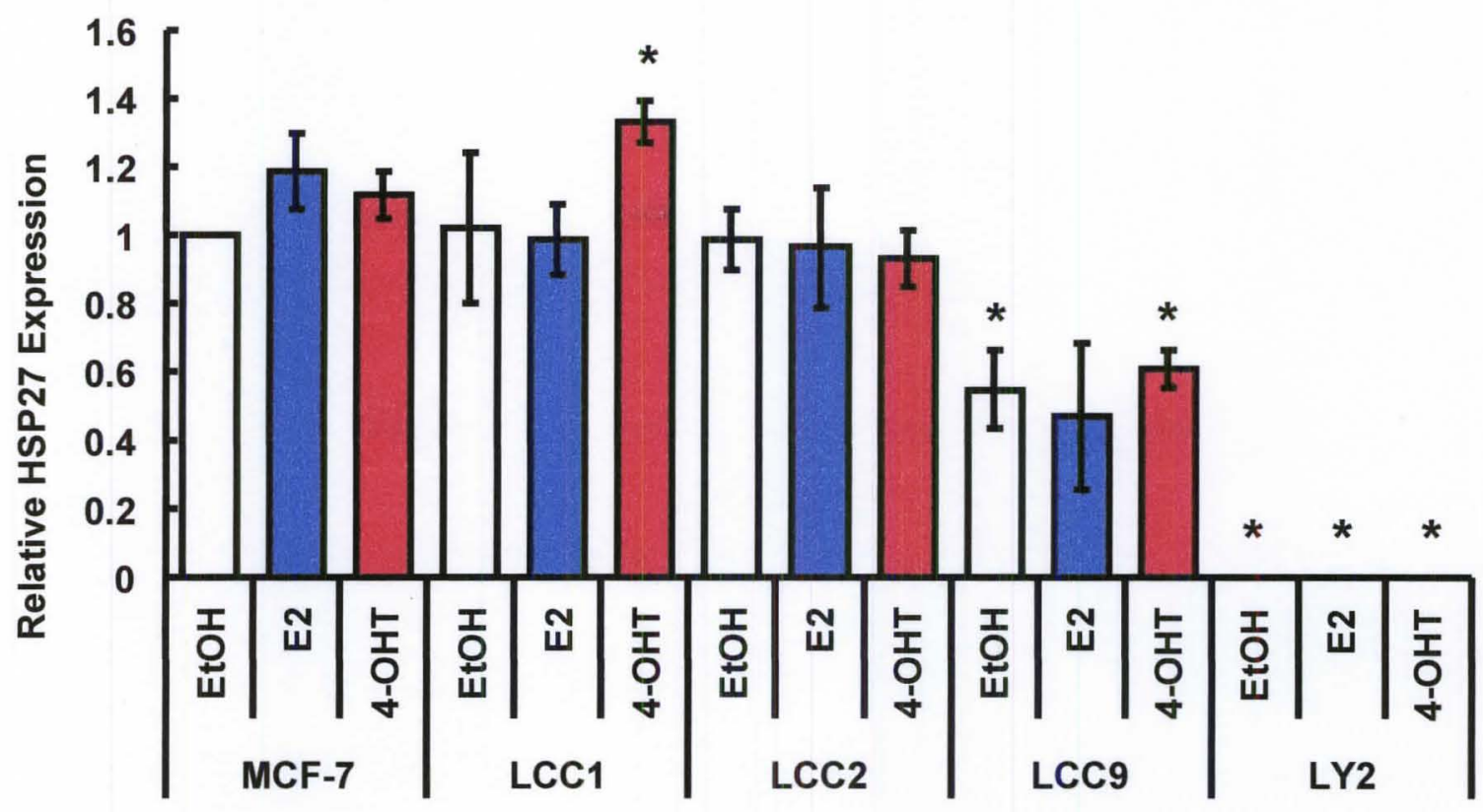

Figure 23. Regulation of HSP27 expression by $\mathrm{E}_{2}$ and 4-OHT in TAMS and TAMR cells.

QRT-PCR for HSPB1 (HSP27) in MCF-7, LCC1, LCC2, LCC9, and LY2 cells treated with $\mathrm{EtOH}, 10 \mathrm{nM} \mathrm{E}$, or $100 \mathrm{nM} 4-\mathrm{OHT}$ for $6 \mathrm{~h}$. Each bar is the average of triplicate determinations in two experiments \pm SEM. * Significantly different $\mathrm{p}<0.05$ from MCF-7 EtOH-treated cells. 
A
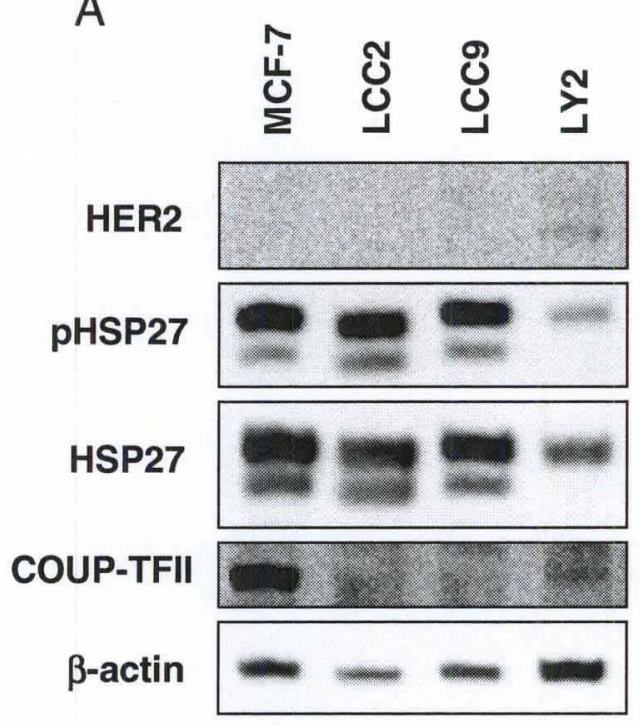

B

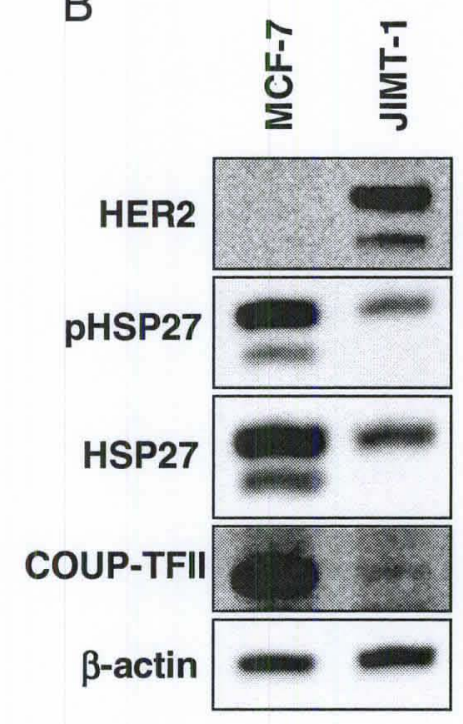

Figure 24. Expression of HSP27, HER2, and COUP-TFII in endocrine-resistant and trastuzumab-resistant cells.

Western blot of WCE (40 $\mu \mathrm{g}$ ) from (A) TAMS MCF-7 and TAMR LCC2, LCC9, and LY2 breast cancer cells or (B) MCF-7 and trastuzumab-resistant JIMT-1 breast cancer cells to evaluate the expression of HER2, phospho-HSP27 (Ser82), HSP27, and COUPTFII relative to $\beta$-actin. 


\section{DISCUSSION}

The role of HSP27 in breast cancer response to antiestrogens and other treatments has been controversial. Examination of HSP27 expression by immunohistochemistry in ER $\alpha+$ tamoxifen-treated breast cancer patients with metastatic disease revealed no statistically significant association between HSP27 and response to tamoxifen therapy [320]. This is in contrast to an early study which reported that patients with HSP27+ tumors responded better to tamoxifen and had a lower failure rate than patients with HSP27- tumors, implicating loss of HSP27 in progression to tamoxifen-resistance [321]. Another group reported increased resistance to doxorubicin in MDA-MB-231 cells overexpressing HSP27 [342]. However, HSP27 expression was reduced in doxorubicinresistant MCF-7/adr cells. This was also correlated to reduced heat shock factor 1 expression and increased NFKB p65 and mutant p53 expression in the MCF-7/adr cells [324]. A trastuzumab-resistant cell line, SKBR3 HR, expresses increased levels of HSP27 compared to the parental SKBR3 cell line. Knockdown of HSP27 expression resulted in increased trastuzumab sensitivity, although no correlation between HSP27 and trastuzumab sensitivity could be established across a panel of cell lines [322]. Further evaluation of the reduced HSP27 expression in endocrine-resistant and trastuzumabresistant breast cancer cells (Fig. 24A and B) and the interaction of HSP27 with COUPTFII (Fig. 22A and B) identified herein may provide more clues. Evaluation of the specific identity of the lower molecular weight band of HSP27 present in MCF-7, LCC2, and LCC9 but not LY2 and JIMT-1 cells (Fig. 24A and B) may also be of interest.

The phosphorylation status of HSP27 may also contribute to its ability to modulate chemoresistance [322,323]. Decreased phosphorylation of HSP27 at Ser82 
with increasing endocrine resistance or trastuzumab resistance corresponding to decreased HSP27 expression was identified in this study (Fig. 24A-B), but phosphorylation of other sites may also be important. Phosphorylation of HSP27 at Ser78 is regulated downstream of the HER2-p38 MAPK pathway [343], and may also play a role in trastuzumab sensitivity. Induction of apoptosis was associated with increased phosphorylation of HSP27 in response to TNF $\alpha$ or 4-OHT treatment, suggesting HSP27 phosphorylation may play a role in treatment response [325]. Phosphorylation of HSP27 also increases its nuclear translocation, while unphosphorylated HSP27 remains distributed throughout the cell [319]. It would be interesting to see if phosphorylation plays a role in HSP27 interaction with COUP-TFII. Much remains to be determined about the role of phosphorylation in HSP27 function and effect on drug sensitivity. Further investigation into whether phosphorylation of HSP27 is tumor-promoting or tumor-suppressive will yield insight into the complex cellular actions of HSP27.

In addition to HSP27, other cancer-associated proteins were found to interact with COUP-TFII through this proteomic study, which will require further investigation. The interaction between COUP-TFII and calmodulin was also verified herein (Fig. 22A). Calmodulin, a calcium binding protein, also interacts with EGFR [344], HER2 [345], $\operatorname{ER} \beta$ [346], and ER $\alpha$ [347,348]. Binding of calmodulin to ER $\alpha$ stabilizes ER $\alpha$ [349], and tamoxifen inhibits the interaction of ER $\alpha$ and calmodulin [350]. Calmodulin appears to play a role in HER2 stimulated SKBR3 cell proliferation since calmodulin inhibition decreased HER2-stimulated cell growth [345]. Further investigation of the function of the interaction between COUP-TFII and calmodulin would be of interest. Also found to 
interact with COUP-TFII was DEAD box protein 5 (DDX5), which interacts with ER $\alpha$ where it functions as a transcriptional coactivator [351]. DDX5 also interacts with other ER $\alpha$ and COUP-TFII coactivators SRC-1, SRC-2, and SRC-3 [352]. Interaction between DDX5 and COUP-TFII has not yet been reported, but would be another potential area to pursue.

In conclusion, novel COUP-TFII-interacting proteins have been identified through a proteomic screen and their relative interaction with COUP-TFII following treatment with EtOH or 4-OHT has been qualitatively analyzed. COUP-TFII-HSP27 interaction in MCF-7 cells and reduced expression of HSP27 in endocrine-resistance and trastuzumab-resistance raises many questions about the function of these proteins in breast cancer cells. Of particular interest will be analyses of HSP27 localization and phosphorylation in endocrine-resistant and trastuzumab-resistant cells, and the effect of COUP-TFII on these properties. 


\section{CHAPTER VI - RESEARCH IMPLICATIONS}

Breast cancer is the most common type of cancer diagnosed in women and the second leading cause of female cancer death [4]. The use of antiestrogen therapy such as selective estrogen receptor modulators (SERMs) greatly increases survival, with five years of tamoxifen therapy reducing mortality rate by $32 \%$ and recurrence rate by $45 \%$ [50]. Despite the initial efficacy of this treatment, approximately $40 \%$ of patients undergoing antiestrogen therapy progress to metastatic disease and die [56]. Identification of novel treatment combinations, biomarkers, and drug targets is of great importance in increasing the effectiveness of antiestrogen therapy for patients. In this study, I have described the function of the orphan nuclear receptor COUP-TFII in breast cancer and endocrine resistance.

COUP-TFII-interacting proteins in MCF-7 breast cancer cells were identified in studies described in Chapter III. The interaction between COUP-TFII and nucleolin was verified through co-immunoprecipitation and an in vitro binding assay [115]. Nucleolin is a multifunctional protein that has roles in mRNA stability, ribosome biogenesis, apoptosis, and the modulation of gene expression as a coregulator [253-257]. As COUPTFII transcriptional action is largely modified by interaction with coregulators [226], a role for nucleolin as a coregulator for transcription of a COUP-TFII-regulated gene retinoic acid receptor B2 (RARB2) was explored. Nucleolin enhanced COUP-TFII 
activated RARB2 expression, and siRNA-mediated knockdown of COUP-TFII or nucleolin reduced RARB2 expression [115]. This indicates that both COUP-TFII and nucleolin are necessary for the basal expression of RARB2. Binding of COUP-TFII to the promoter of RARB2 was also verified, as well as COUP-TFII regulation of a RARB2-target gene, retinoid receptor-induced gene-1 (RRIG1) [115]. Supporting evidence for the importance of COUP-TFII in breast cancer was provided by immunohistochemical (IHC) staining of breast cancer tumor tissue microarrays, where an inverse correlation was found between COUP-TFII expression and tumor grade. COUP-TFII and nucleolin expression were also positively correlated in ER $\alpha+$ invasive ductal carcinomas [115]. These data enhance our understanding of the regulation of COUP-TFII's transcriptional activity, as well as the characteristics of COUP-TFII expression in breast cancer tissue.

Several reports have implicated increased NFKB activity as a driving pathway in endocrine-resistance breast cancer [87,88,280-284]. As COUP-TFII expression is reduced in endocrine-resistant breast cancer, and COUP-TFII can repress the activity of other transcription factors, Chapter IV explores a role for COUP-TFII in suppression of NFkB activity in breast cancer. Endocrine-resistant LCC9 cells displayed much greater basal and $\mathrm{TNF} \alpha$-induced $\mathrm{NFKB}$ activity than endocrinesensitive MCF-7 cells. Transfection of COUP-TFII in endocrine-resistant LCC9 cells reduced NFKB activation. COUP-TFII suppressed both the basal and TNF $\alpha$-induced expression of NFKB target genes IL6, CCL2, ICAM1, and TNFAIP3 in LCC9 cells, as well as the basal expression of ICAM1 and TNFAIP3 and the TNF $\alpha$-induced CCL2 and TNFAIP3 in MCF-7 cells. COUP-TFII also suppressed the basal expression of 
the REL and RELA NFKB subunits in LCC9 cells and the TNF $\alpha$-induced expression of NFKB1, REL, and RELB in LCC9. As the NFkB subunits are also downstream targets of the NFאB pathway themselves [306-309], this provides further evidence of the decreased NFKB activation with COUP-TFII transfection. The correlation between COUP-TFII and NFאB target gene and subunit expression was further examined through analysis of data from a microarray of gene expression in breast tumors from $\mathrm{ER} \alpha+$ breast cancer patients treated with tamoxifen. Consistent with the data in Chapter IV, an inverse correlation was found between COUP-TFII and ICAM1, IL6, TNFAIP3, NFKB2, REL, RELA, and RELB in the patient samples. This suggests that the same trend seen in the breast cancer cells is also true for breast cancer patients.

To further investigate the mechanism of COUP-TFII regulation of NFkB activation, the impact of COUP-TFII on NFאB DNA-binding was analyzed through EMSA. COUP-TFII suppressed DNA-binding in both an in vitro assay where in vitro transcribed/translated COUP-TFII was incubated with nuclear extract from LCC9 cells treated with TNFo, as well as in an EMSA with nuclear extract from MCF-7 and LCC9 cells transfected with COUP-TFII. Further, co-immunoprecipitation revealed that COUP-TFII interacts with NFkB subunits RelB and NFkB1. This suggests COUP-TFII interaction with NFאB subunits inhibits binding to the NFאB

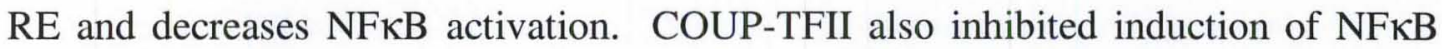
activity by coactivators SRC-1, SRC-3, and CBP, implying that COUP-TFII and NFkB compete for these coactivators, a mechanism that has been recently reported for COUP-TFII action on other transcription factors [295]. 
Identification of COUP-TFII as a negative regulator of NFKB activity in breast cancer cells provides valuable information about the possible mechanism by which COUP-TFII helps prevent the progression to endocrine-resistance. It may partly explain the ability of COUP-TFII to increase endocrine sensitivity [116], as well as why endocrine-resistant cells (which have lower COUP-TFII expression) have induced NFKB activity. These data also point to future use for COUP-TFII as both a biomarker as well as a target for the enhancement of endocrine sensitivity. LCC9 endocrine-resistant cells were found to be more sensitive to treatment with an NFKB inhibitor, and showed markedly reduced cell proliferation as compared to MCF-7 cells. This may be due to their increased dependence on the NFKB pathway for cell survival, and may provide a target for therapeutic intervention.

Chapters III and IV have highlighted the importance of interacting proteins on COUP-TFII function. The focus of the experiments described in Chapter V is to identify proteins which differentially interact with COUP-TFII upon treatment with tamoxifen. Many proteins were identified which could provide a starting point for future studies. DAVID analysis was used to identify common themes amongst the interacting proteins, including RNA splicing, calmodulin-binding, and ATP-binding. As calmodulin was also found in the interacting proteins with $\mathrm{EtOH}$ (vehicle control) treatment, and verified through co-immunoprecipitation in Chapter V, this could be an interesting pathway for further investigation.

Also in Chapter V, the interaction between COUP-TFII and HSP27 was verified through co-immunoprecipitation. Despite reports that HSP27 increase cell proliferation and plays an oncogenic role in cancer progression [335-337], reduced 
HSP27 expression was found with increasing endocrine-resistance. Reduced HSP27 expression was also present in JIMT-1 trastuzumab-resistant breast cancer cells. Conflicting reports have identified HSP27 as both increasing [322,342] and decreasing drug resistance $[321,324]$, and the interaction and positive correlation of COUP-TFII and HSP27 merits further study. Phosphorylation of HSP27 at Serine 15, 78 , and 82 also plays a role in cellular localization and function [319,325], and has not been widely studied in resistance to antiestrogens or other drugs. A correlation of HSP27 phosphorylation at Ser82 and drug resistance was not found in the endocrineresistant and trastuzumab-resistant cell lines studied in Chapter V, but further studies will investigate phosphorylation at other serines. Subcellular localization of HSP27 in these cell lines will also be examined.

Overall, this study has provided important new information about the many roles of COUP-TFII in breast cancer and confirmed the importance of COUP-TFIIinteracting proteins to its cellular function. Further investigation of the mechanisms responsible for the ability of COUP-TFII to enhance endocrine sensitivity is crucial for its use as a biomarker or therapeutic target. Identification of biomarkers to enable detection of patients who would respond favorably to endocrine therapy would be of great use, as would discovery of a target to reduce endocrine resistance in patients. 


\section{REFERENCES}

1. Brisken C, O'Malley B (2010) Hormone action in the mammary gland. Cold Spring Harb Perspect Biol 2: a003178.

2. Petersen OW, Polyak K (2010) Stem cells in the human breast. Cold Spring Harb Perspect Biol 2: a003160.

3. Kotsopoulos J, Chen WY, Gates MA, Tworoger SS, Hankinson SE, et al. (2010) Risk factors for ductal and lobular breast cancer: results from the nurses' health study. Breast Cancer Res 12: R106.

4. Siegel R, Naishadham D, Jemal A (2013) Cancer statistics, 2013. CA Cancer J Clin 63: 11-30.

5. Sorlie T, Perou CM, Tibshirani R, Aas T, Geisler S, et al. (2001) Gene expression patterns of breast carcinomas distinguish tumor subclasses with clinical implications. Proc Natl Acad Sci U S A 98: 10869-10874.

6. Sorlie T, Tibshirani R, Parker J, Hastie T, Marron JS, et al. (2003) Repeated observation of breast tumor subtypes in independent gene expression data sets. Proc Natl Acad Sci U S A 100: 8418-8423.

7. Perou CM, Sorlie T, Eisen MB, van de Rijn M, Jeffrey SS, et al. (2000) Molecular portraits of human breast tumours. Nature 406: 747-752.

8. Cheang MC, Chia SK, Voduc D, Gao D, Leung S, et al. (2009) Ki67 index, HER2 status, and prognosis of patients with luminal B breast cancer. J Natl Cancer Inst 101: 736-750.

9. Voduc KD, Cheang MC, Tyldesley S, Gelmon K, Nielsen TO, et al. (2010) Breast cancer subtypes and the risk of local and regional relapse. J Clin Oncol 28: 1684-1691.

10. Colleoni M, Rotmensz N, Peruzzotti G, Maisonneuve P, Viale G, et al. (2004) Minimal and small size invasive breast cancer with no axillary lymph node involvement: the need for tailored adjuvant therapies. Ann Oncol 15: 16331639.

11. Elkhuizen PH, Voogd AC, van den Broek LC, Tan IT, van Houwelingen HC, et al. (1999) Risk factors for local recurrence after breast-conserving therapy for invasive carcinomas: a case-control study of histological factors and alterations in oncogene expression. Int J Radiat Oncol Biol Phys 45: 73-83.

12. Arteaga CL, Sliwkowski MX, Osborne CK, Perez EA, Puglisi F, et al. (2012) Treatment of HER2-positive breast cancer: current status and future perspectives. Nat Rev Clin Oncol 9: 16-32.

13. Allen E, Doisy EA (1983) Landmark article Sept 8, 1923. An ovarian hormone. Preliminary report on its localization, extraction and partial purification, and 
action in test animals. By Edgar Allen and Edward A. Doisy. JAMA 250: 2681-2683.

14. Coelingh Bennink HJT (2004) Are all estrogens the same? Maturitas 47: 269-275.

15. Jensen EV, Jordan VC (2003) The estrogen receptor: a model for molecular medicine. Clin Cancer Res 9: 1980-1989.

16. Kuiper GG, Enmark E, Pelto-Huikko M, Nilsson S, Gustafsson JA (1996) Cloning of a novel receptor expressed in rat prostate and ovary. Proc Natl Acad Sci U S A 93: 5925-5930.

17. Klinge CM (2001) Estrogen receptor interaction with estrogen response elements. Nucleic Acids Res 29: 2905-2919.

18. Klinge CM (2003) Estrogen response element sequence as an allosteric regulator of estrogen receptor action. ChemTracts Biochem Molec Biol 16: 1-18.

19. Uht RM, Webb P, Nguyen P, Price Jr RH, Jr., Valentine C, et al. (2004) A conserved lysine in the estrogen receptor DNA binding domain regulates ligand activation profiles at AP-1 sites, possibly by controlling interactions with a modulating repressor. Nucl Recept 2: 2 .

20. Johnston SR, Lu B, Scott GK, Kushner PJ, Smith IE, et al. (1999) Increased activator protein-1 DNA binding and c-Jun NH2-terminal kinase activity in human breast tumors with acquired tamoxifen resistance. Clin Cancer Res 5: 251-256.

21. Nilsson S, Makela S, Treuter E, Tujague M, Thomsen J, et al. (2001) Mechanisms of estrogen action. Physiol Rev 81: 1535-1565.

22. Safe S (2001) Transcriptional activation of genes by 17 beta-estradiol through estrogen receptor-Sp1 interactions. Vitam Horm 62: 231-252.

23. Klinge CM (2000) Estrogen receptor interaction with co-activators and corepressors. Steroids 65: 227-251.

24. Carroll JS, Meyer CA, Song J, Li W, Geistlinger TR, et al. (2006) Genome-wide analysis of estrogen receptor binding sites. Nat Genet 38: 1289-1297.

25. Carroll JS, Brown M (2006) Estrogen receptor target gene: an evolving concept. Mol Endocrinol 20: 1707-1714.

26. Frasor J, Danes JM, Komm B, Chang KC, Lyttle CR, et al. (2003) Profiling of estrogen up- and down-regulated gene expression in human breast cancer cells: insights into gene networks and pathways underlying estrogenic control of proliferation and cell phenotype. Endocrinology 144: 4562-4574.

27. Charn TH, Liu ET, Chang EC, Lee YK, Katzenellenbogen JA, et al. (2010) Genome-wide dynamics of chromatin binding of estrogen receptors alpha and beta: mutual restriction and competitive site selection. Mol Endocrinol 24: 4759.

28. Nilsson S, Koehler KF, Gustafsson JA (2011) Development of subtype-selective oestrogen receptor-based therapeutics. Nat Rev Drug Discov 10: 778-792.

29. Thomas C, Gustafsson JA (2011) The different roles of ER subtypes in cancer biology and therapy. Nat Rev Cancer 11: 597-608.

30. Meyers MJ, Sun J, Carlson KE, Marriner GA, Katzenellenbogen BS, et al. (2001) Estrogen receptor-beta potency-selective ligands: structure-activity relationship studies of diarylpropionitriles and their acetylene and polar analogues. J Med Chem 44: 4230-4251. 
31. Leitman DC, Paruthiyil S, Vivar OI, Saunier EF, Herber CB, et al. (2010) Regulation of specific target genes and biological responses by estrogen receptor subtype agonists. Curr Opin Pharmacol 10: 629-636.

32. Thomas C, Rajapaksa G, Nikolos F, Hao R, Katchy A, et al. (2012) ERbeta1 represses basal-like breast cancer epithelial to mesenchymal transition by destabilizing EGFR. Breast Cancer Res 14: R148.

33. Jordan VC (2001) Selective estrogen receptor modulation: a personal perspective. Cancer Res 61: 5683-5687.

34. Dowsett M, Nicholson RI, Pietras RJ (2005) Biological characteristics of the pure antiestrogen fulvestrant: overcoming endocrine resistance. Breast Cancer Res Treat 93 Suppl 1: S11-18.

35. Chumsri S, Howes T, Bao T, Sabnis G, Brodie A (2011) Aromatase, aromatase inhibitors, and breast cancer. J Steroid Biochem Mol Biol 125: 13-22.

36. Boon WC, Chow JD, Simpson ER (2010) The multiple roles of estrogens and the enzyme aromatase. Prog Brain Res 181: 209-232.

37. Lacassagne A (1936) A Comparative Study of the Carcinogenic Action of Certain Oestrogenic Hormones. The American Journal of Cancer 28: 735-740.

38. Jensen EV, Block GE, Smith S, Kyser K, DeSombre ER (1971) Estrogen receptors and breast cancer response to adrenalectomy. Natl Cancer Inst Monogr 34: 55-70.

39. Harper MJ, Walpole AL (1966) Contrasting endocrine activities of cis and trans isomers in a series of substituted triphenylethylenes. Nature 212: 87.

40. Harper MJ, Walpole AL (1967) Mode of action of I.C.I. 46,474 in preventing implantation in rats. J Endocrinol 37: 83-92.

41. Shiau AK, Barstad D, Loria PM, Cheng L, Kushner PJ, et al. (1998) The structural basis of estrogen receptor/coactivator recognition and the antagonism of this interaction by tamoxifen. Cell 95: 927-937.

42. Shang Y, Brown M (2002) Molecular determinants for the tissue specificity of SERMs. Science 295: 2465-2468.

43. Smith CL, O'Malley BW (2004) Coregulator function: a key to understanding tissue specificity of selective receptor modulators. Endocr Rev 25: 45-71.

44. Smith CL, Nawaz Z, O'Malley BW (1997) Coactivator and corepressor regulation of the agonist/antagonist activity of the mixed antiestrogen, 4hydroxytamoxifen. Mol Endocrinol 11: 657-666.

45. Salami S, Karami-Tehrani F (2003) Biochemical studies of apoptosis induced by tamoxifen in estrogen receptor positive and negative breast cancer cell lines. Clin Biochem 36: 247-253.

46. Mandlekar S, Kong AN (2001) Mechanisms of tamoxifen-induced apoptosis. Apoptosis 6: 469-477.

47. Fisher B, Costantino JP, Redmond CK, Fisher ER, Wickerham DL, et al. (1994) Endometrial cancer in tamoxifen-treated breast cancer patients: findings from the National Surgical Adjuvant Breast and Bowel Project (NSABP) B-14. J Natl Cancer Inst 86: 527-537.

48. Jordan VC (2009) A century of deciphering the control mechanisms of sex steroid action in breast and prostate cancer: the origins of targeted therapy and chemoprevention. Cancer Res 69: 1243-1254. 
49. Early Breast Cancer Trialists' Collaborative G (2005) Effects of chemotherapy and hormonal therapy for early breast cancer on recurrence and 15-year survival: an overview of the randomised trials. Lancet 365: 1687-1717.

50. Brauch H, Jordan VC (2009) Targeting of tamoxifen to enhance antitumour action for the treatment and prevention of breast cancer: the 'personalised' approach? European journal of cancer 45: 2274-2283.

51. Wijayaratne AL, McDonnell DP (2001) The human estrogen receptor-alpha is a ubiquitinated protein whose stability is affected differentially by agonists, antagonists, and selective estrogen receptor modulators. J Biol Chem 276: 35684-35692.

52. Schwarzel WC, Kruggel WG, Brodie HJ (1973) Studies on the mechanism of estrogen biosynthesis. 8 . The development of inhibitors of the enzyme system in human placenta. Endocrinology 92: 866-880.

53. Killinger DW, Perel E, Daniilescu D, Kharlip L, Blackstein ME (1987) Aromatase activity in the breast and other peripheral tissues and its therapeutic regulation. Steroids 50: 523-536.

54. Sasano H, Miki Y, Nagasaki S, Suzuki T (2009) In situ estrogen production and its regulation in human breast carcinoma: from endocrinology to intracrinology. Pathol Int 59: 777-789.

55. Regan MM, Price KN, Giobbie-Hurder A, Thurlimann B, Gelber RD, et al. (2011) Interpreting Breast International Group (BIG) 1-98: a randomized, double-blind, phase III trial comparing letrozole and tamoxifen as adjuvant endocrine therapy for postmenopausal women with hormone receptorpositive, early breast cancer. Breast Cancer Res 13: 209.

56. Ring A, Dowsett M (2004) Mechanisms of tamoxifen resistance. Endocr Relat Cancer 11: 643-658.

57. Gutierrez MC, Detre S, Johnston S, Mohsin SK, Shou J, et al. (2005) Molecular changes in tamoxifen-resistant breast cancer: relationship between estrogen receptor, HER-2, and p38 mitogen-activated protein kinase. J Clin Oncol 23: 2469-2476.

58. Shi L, Dong B, Li Z, Lu Y, Ouyang T, et al. (2009) Expression of ER-\{alpha 36 , a novel variant of estrogen receptor $\{$ alpha $\}$, and resistance to tamoxifen treatment in breast cancer. J Clin Oncol 27: 3423-3429.

59. Osborne CK, Bardou V, Hopp TA, Chamness GC, Hilsenbeck SG, et al. (2003) Role of the estrogen receptor coactivator AIB1 (SRC-3) and HER-2/neu in tamoxifen resistance in breast cancer. J Natl Cancer Inst 95: 353-361.

60. McIlroy M, McCartan D, Early S, P OG, Pennington S, et al. (2010) Interaction of developmental transcription factor HOX11 with steroid receptor coactivator SRC-1 mediates resistance to endocrine therapy in breast cancer. Cancer Res 70: 1585-1594.

61. Redmond AM, Bane FT, Stafford AT, McIlroy M, Dillon MF, et al. (2009) Coassociation of estrogen receptor and p160 proteins predicts resistance to endocrine treatment; SRC-1 is an independent predictor of breast cancer recurrence. Clin Cancer Res 15: 2098-2106. 
62. Lonard DM, Tsai SY, O'Malley BW (2004) Selective estrogen receptor modulators 4-hydroxytamoxifen and raloxifene impact the stability and function of SRC-1 and SRC-3 coactivator proteins. Mol Cell Biol 24: 14-24.

63. Lavinsky RM, Jepsen K, Heinzel T, Torchia J, Mullen TM, et al. (1998) Diverse signaling pathways modulate nuclear receptor recruitment of $\mathrm{N}-\mathrm{CoR}$ and SMRT complexes. Proc Natl Acad Sci U S A 95: 2920-2925.

64. Girault I, Lerebours F, Amarir S, Tozlu S, Tubiana-Hulin M, et al. (2003) Expression analysis of estrogen receptor alpha coregulators in breast carcinoma: evidence that NCOR1 expression is predictive of the response to tamoxifen. Clin Cancer Res 9: 1259-1266.

65. Smith CL, Migliaccio I, Chaubal V, Wu MF, Pace MC, et al. (2012) Elevated nuclear expression of the SMRT corepressor in breast cancer is associated with earlier tumor recurrence. Breast Cancer Res Treat 136: 253-265.

66. Pancholi S, Lykkesfeldt AE, Hilmi C, Banerjee S, Leary A, et al. (2008) ERBB2 influences the subcellular localization of the estrogen receptor in tamoxifenresistant MCF-7 cells leading to the activation of AKT and RPS6KA2. Endocr Relat Cancer 15: 985-1002.

67. Chung YL, Sheu ML, Yang SC, Lin CH, Yen SH (2002) Resistance to tamoxifeninduced apoptosis is associated with direct interaction between Her2/neu and cell membrane estrogen receptor in breast cancer. Int J Cancer 97: 306-312.

68. Weinberg OK, Marquez-Garban DC, Pietras RJ (2005) New approaches to reverse resistance to hormonal therapy in human breast cancer. Drug resistance updates : reviews and commentaries in antimicrobial and anticancer chemotherapy 8: 219-233.

69. Pietras RJ, Marquez-Garban DC (2007) Membrane-associated estrogen receptor signaling pathways in human cancers. Clin Cancer Res 13: 4672-4676.

70. Pietras RJ, Arboleda J, Reese DM, Wongvipat N, Pegram MD, et al. (1995) HER2 tyrosine kinase pathway targets estrogen receptor and promotes hormoneindependent growth in human breast cancer cells. Oncogene 10: 2435-2446.

71. Acconcia F, Ascenzi P, Bocedi A, Spisni E, Tomasi V, et al. (2005) Palmitoylation-dependent estrogen receptor alpha membrane localization: regulation by 17beta-estradiol. Mol Biol Cell 16: 231-237.

72. Pedram A, Razandi M, Sainson RC, Kim JK, Hughes CC, et al. (2007) A conserved mechanism for steroid receptor translocation to the plasma membrane. J Biol Chem 282: 22278-22288.

73. Marquez DC, Chen HW, Curran EM, Welshons WV, Pietras RJ (2006) Estrogen receptors in membrane lipid rafts and signal transduction in breast cancer. Mol Cell Endocrinol 246: 91-100.

74. Fan P, Wang J, Santen RJ, Yue W (2007) Long-term treatment with tamoxifen facilitates translocation of estrogen receptor alpha out of the nucleus and enhances its interaction with EGFR in MCF-7 breast cancer cells. Cancer Res 67: 1352-1360.

75. Kahlert S, Nuedling S, van Eickels M, Vetter H, Meyer R, et al. (2000) Estrogen receptor alpha rapidly activates the IGF-1 receptor pathway. J Biol Chem 275: 18447-18453. 
76. Knowlden JM, Hutcheson IR, Jones HE, Madden T, Gee JM, et al. (2003) Elevated levels of epidermal growth factor receptor/c-erbB2 heterodimers mediate an autocrine growth regulatory pathway in tamoxifen-resistant MCF7 cells. Endocrinology 144: 1032-1044.

77. Shou J, Massarweh S, Osborne CK, Wakeling AE, Ali S, et al. (2004) Mechanisms of tamoxifen resistance: increased estrogen receptor-HER2/neu cross-talk in ER/HER2-positive breast cancer. J Natl Cancer Inst 96: 926-935.

78. Nicholson RI, Hutcheson IR, Harper ME, Knowlden JM, Barrow D, et al. (2001) Modulation of epidermal growth factor receptor in endocrine-resistant, oestrogen receptor-positive breast cancer. Endocr Relat Cancer 8: 175-182.

79. Michie AM, Nakagawa R (2005) The link between PKCalpha regulation and cellular transformation. Immunology letters 96: 155-162.

80. Frankel LB, Lykkesfeldt AE, Hansen JB, Stenvang J (2007) Protein Kinase C alpha is a marker for antiestrogen resistance and is involved in the growth of tamoxifen resistant human breast cancer cells. Breast Cancer Res Treat 104: 165-179.

81. Simoncini T, Hafezi-Moghadam A, Brazil DP, Ley K, Chin WW, et al. (2000) Interaction of oestrogen receptor with the regulatory subunit of phosphatidylinositol-3-OH kinase. Nature 407: 538-541.

82. Campbell RA, Bhat-Nakshatri P, Patel NM, Constantinidou D, Ali S, et al. (2001) Phosphatidylinositol 3-kinase/AKT-mediated activation of estrogen receptor alpha: a new model for anti-estrogen resistance. J Biol Chem 276: 9817-9824.

83. Clark AS, West K, Streicher S, Dennis PA (2002) Constitutive and inducible Akt activity promotes resistance to chemotherapy, trastuzumab, or tamoxifen in breast cancer cells. Molecular cancer therapeutics 1: 707-717.

84. Ahmad N, Kumar R (2011) Steroid hormone receptors in cancer development: a target for cancer therapeutics. Cancer Lett 300: 1-9.

85. Muscat GE, Eriksson NA, Byth K, Loi S, Graham D, et al. (2013) Research resource: nuclear receptors as transcriptome: discriminant and prognostic value in breast cancer. Mol Endocrinol 27: 350-365.

86. Prasad S, Ravindran J, Aggarwal BB (2010) NF-kappaB and cancer: how intimate is this relationship. Mol Cell Biochem 336: 25-37.

87. Nehra R, Riggins RB, Shajahan AN, Zwart A, Crawford AC, et al. (2010) BCL2 and CASP8 regulation by NF-kappaB differentially affect mitochondrial function and cell fate in antiestrogen-sensitive and -resistant breast cancer cells. FASEB J 24: 2040-2055.

88. Riggins RB, Zwart A, Nehra R, Clarke R (2005) The nuclear factor kappa B inhibitor parthenolide restores ICI 182,780 (Faslodex; fulvestrant)-induced apoptosis in antiestrogen-resistant breast cancer cells. Mol Cancer Ther 4: 3341.

89. Zhou Y, Eppenberger-Castori S, Eppenberger U, Benz CC (2005) The NFkappaB pathway and endocrine-resistant breast cancer. Endocrine-related cancer 12 Suppl 1: S37-46.

90. deGraffenried LA, Chandrasekar B, Friedrichs WE, Donzis E, Silva J, et al. (2004) NF-kappa B inhibition markedly enhances sensitivity of resistant breast cancer tumor cells to tamoxifen. Ann Oncol 15: 885-890. 
91. Yde CW, Emdal KB, Guerra B, Lykkesfeldt AE (2012) NFkappaB signaling is important for growth of antiestrogen resistant breast cancer cells. Breast Cancer Res Treat 135: 67-78.

92. Nakshatri H, Bhat-Nakshatri P, Martin DA, Goulet RJ, Jr., Sledge GW, Jr. (1997) Constitutive activation of NF-kappaB during progression of breast cancer to hormone-independent growth. Mol Cell Biol 17: 3629-3639.

93. Pratt MA, Bishop TE, White D, Yasvinski G, Menard M, et al. (2003) Estrogen withdrawal-induced NF-kappaB activity and bcl-3 expression in breast cancer cells: roles in growth and hormone independence. Molecular and cellular biology 23: 6887-6900.

94. Dumont JA, Bitonti AJ, Wallace CD, Baumann RJ, Cashman EA, et al. (1996) Progression of MCF-7 breast cancer cells to antiestrogen-resistant phenotype is accompanied by elevated levels of AP-1 DNA-binding activity. Cell Growth Differ 7: 351-359.

95. Smith LM, Wise SC, Hendricks DT, Sabichi AL, Bos T, et al. (1999) cJun overexpression in MCF-7 breast cancer cells produces a tumorigenic, invasive and hormone resistant phenotype. Oncogene 18: 6063-6070.

96. Lovat F, Valeri N, Croce CM (2011) MicroRNAs in the pathogenesis of cancer. Semin Oncol 38: 724-733.

97. Adams BD, Furneaux H, White BA (2007) The micro-ribonucleic acid (miRNA) miR-206 targets the human estrogen receptor-alpha (ERalpha) and represses ERalpha messenger RNA and protein expression in breast cancer cell lines. Mol Endocrinol 21: 1132-1147.

98. Zhao JJ, Lin J, Yang H, Kong W, He L, et al. (2008) MicroRNA-221/222 negatively regulates estrogen receptor alpha and is associated with tamoxifen resistance in breast cancer. J Biol Chem 283: 31079-31086.

99. Miller TE, Ghoshal K, Ramaswamy B, Roy S, Datta J, et al. (2008) MicroRNA221/222 confers tamoxifen resistance in breast cancer by targeting p27Kip1. J Biol Chem 283: 29897-29903.

100. Manavalan TT, Teng Y, Appana SN, Datta S, Kalbfleisch TS, et al. (2011) Differential expression of microRNA expression in tamoxifen-sensitive MCF7 versus tamoxifen-resistant LY2 human breast cancer cells. Cancer Lett 313: 26-43.

101. Fan M, Yan PS, Hartman-Frey C, Chen L, Paik H, et al. (2006) Diverse gene expression and DNA methylation profiles correlate with differential adaptation of breast cancer cells to the antiestrogens tamoxifen and fulvestrant. Cancer research 66: 11954-11966.

102. Jordan VC, Obiorah I, Fan P, Kim HR, Ariazi E, et al. (2011) The St. Gallen Prize Lecture 2011: evolution of long-term adjuvant anti-hormone therapy: consequences and opportunities. Breast 20 Suppl 3: S1-11.

103. Siddikuzzaman, Guruvayoorappan C, Berlin Grace VM (2011) All trans retinoic acid and cancer. Immunopharmacol Immunotoxicol 33: 241-249.

104. Risbridger GP, Davis ID, Birrell SN, Tilley WD (2010) Breast and prostate cancer: more similar than different. Nat Rev Cancer 10: 205-212.

105. Stanisic V, Lonard DM, O'Malley BW (2010) Modulation of steroid hormone receptor activity. Prog Brain Res 181: 153-176. 
106. Sagami I, Tsai SY, Wang H, Tsai MJ, O'Malley BW (1986) Identification of two factors required for transcription of the ovalbumin gene. Mol Cell Biol 6: 4259-4267.

107. Tsai SY, Tsai MJ (1997) Chick ovalbumin upstream promoter-transcription factors (COUP-TFs): coming of age. Endocr Rev 18: 229-240.

108. Lin FJ, Qin J, Tang K, Tsai SY, Tsai MJ (2011) Coup d'Etat: An Orphan Takes Control. Endocr Rev.

109. Thornton JW (2001) Evolution of vertebrate steroid receptors from an ancestral estrogen receptor by ligand exploitation and serial genome expansions. Proc Natl Acad Sci U S A 98: 5671-5676.

110. Thornton JW, Need E, Crews D (2003) Resurrecting the ancestral steroid receptor: ancient origin of estrogen signaling. Science 301: 1714-1717.

111. Pereira FA, Qiu Y, Zhou G, Tsai MJ, Tsai SY (1999) The orphan nuclear receptor COUP-TFII is required for angiogenesis and heart development. Genes Dev 13: 1037-1049.

112. Lee CT, Li L, Takamoto N, Martin JF, Demayo FJ, et al. (2004) The nuclear orphan receptor COUP-TFII is required for limb and skeletal muscle development. Molecular and cellular biology 24: 10835-10843.

113. Vasyutina E, Birchmeier C (2006) The development of migrating muscle precursor cells. Anat Embryol (Berl) 211 Suppl 1: 37-41.

114. Zhang LJ, Liu X, Gafken PR, Kioussi C, Leid M (2009) A chicken ovalbumin upstream promoter transcription factor I (COUP-TFI) complex represses expression of the gene encoding tumor necrosis factor alpha-induced protein 8 (TNFAIP8). The Journal of biological chemistry 284: 6156-6168.

115. Litchfield LM, Riggs KA, Hockenberry AM, Oliver LD, Barnhart KG, et al. (2012) Identification and Characterization of Nucleolin as a COUP-TFII Coactivator of Retinoic Acid Receptor beta Transcription in Breast Cancer Cells. PLoS One 7: e38278.

116. Riggs KA, Wickramasinghe NS, Cochrum RK, Watts MB, Klinge CM (2006) Decreased chicken ovalbumin upstream promoter transcription factor II expression in tamoxifen-resistant breast cancer cells. Cancer Res 66: 1018810198.

117. Kliewer SA, Umesono K, Heyman RA, Mangelsdorf DJ, Dyck JA, et al. (1992) Retinoid X receptor-COUP-TF interactions modulate retinoic acid signaling. Proc Natl Acad Sci U S A 89: 1448-1452.

118. Lin B, Chen GQ, Xiao D, Kolluri SK, Cao X, et al. (2000) Orphan receptor COUP-TF is required for induction of retinoic acid receptor beta, growth inhibition, and apoptosis by retinoic acid in cancer cells. Mol Cell Biol 20: 957-970.

119. De Martino MU, Bhattachryya N, Alesci S, Ichijo T, Chrousos GP, et al. (2004) The glucocorticoid receptor and the orphan nuclear receptor chicken ovalbumin upstream promoter-transcription factor II interact with and mutually affect each other's transcriptional activities: implications for intermediary metabolism. Molecular endocrinology 18: 820-833. 
120. Kruse SW, Suino-Powell K, Zhou XE, Kretschman JE, Reynolds R, et al. (2008) Identification of COUP-TFII orphan nuclear receptor as a retinoic acidactivated receptor. PLoS Biol 6: e227.

121. Pipaon C, Tsai SY, Tsai MJ (1999) COUP-TF upregulates NGFI-A gene expression through an Sp1 binding site. Mol Cell Biol 19: 2734-2745.

122. Stroup D, Chiang JY (2000) HNF4 and COUP-TFII interact to modulate transcription of the cholesterol 7alpha-hydroxylase gene (CYP7A1). J Lipid Res 41: 1-11.

123. Marcus SL, Winrow CJ, Capone JP, Rachubinski RA (1996) A p56(lck) ligand serves as a coactivator of an orphan nuclear hormone receptor. The Journal of biological chemistry 271: 27197-27200.

124. De Martino MU, Alesci S, Chrousos GP, Kino T (2004) Interaction of the glucocorticoid receptor and the chicken ovalbumin upstream promotertranscription factor II (COUP-TFII): implications for the actions of glucocorticoids on glucose, lipoprotein, and xenobiotic metabolism. Annals of the New York Academy of Sciences 1024: 72-85.

125. Tang K, Xie X, Park JI, Jamrich M, Tsai S, et al. (2010) COUP-TFs regulate eye development by controlling factors essential for optic vesicle morphogenesis. Development 137: 725-734.

126. Bailey PJ, Dowhan DH, Franke K, Burke LJ, Downes M, et al. (1997) Transcriptional repression by COUP-TF II is dependent on the C-terminal domain and involves the N-CoR variant, RIP13delta1. J Steroid Biochem Mol Biol 63: 165-174.

127. Shibata H, Nawaz Z, Tsai SY, O'Malley BW, Tsai MJ (1997) Gene silencing by chicken ovalbumin upstream promoter-transcription factor I (COUP-TFI) is mediated by transcriptional corepressors, nuclear receptor-corepressor $(\mathrm{N}$ $\mathrm{CoR}$ ) and silencing mediator for retinoic acid receptor and thyroid hormone receptor (SMRT). Mol Endocrinol 11: 714-724.

128. Okamura M, Kudo H, Wakabayashi K, Tanaka T, Nonaka A, et al. (2009) COUP-TFII acts downstream of Wnt/beta-catenin signal to silence PPARgamma gene expression and repress adipogenesis. Proc Natl Acad Sci U S A 106: 5819-5824.

129. Chu K, Zingg HH (1997) The nuclear orphan receptors COUP-TFII and Ear-2 act as silencers of the human oxytocin gene promoter. Journal of molecular endocrinology 19: 163-172.

130. Klinge CM, Silver BF, Driscoll MD, Sathya G, Bambara RA, et al. (1997) Chicken ovalbumin upstream promoter-transcription factor interacts with estrogen receptor, binds to estrogen response elements and half-sites, and inhibits estrogen-induced gene expression. J Biol Chem 272: 31465-31474.

131. Lin F, Kolluri SK, Chen GQ, Zhang XK (2002) Regulation of retinoic acidinduced inhibition of AP-1 activity by orphan receptor chicken ovalbumin upstream promoter-transcription factor. The Journal of biological chemistry 277: 21414-21422.

132. Lee KN, Jang WG, Kim EJ, Oh SH, Son HJ, et al. (2012) Orphan nuclear receptor COUP-TFII negatively regulates BMP2-induced osteoblast differentiation through suppressing Runx2 activity. J Biol Chem. 
133. Cooney AJ, Leng X, Tsai SY, O'Malley BW, Tsai MJ (1993) Multiple mechanisms of chicken ovalbumin upstream promoter transcription factordependent repression of transactivation by the vitamin $\mathrm{D}$, thyroid hormone, and retinoic acid receptors. The Journal of biological chemistry 268: 41524160.

134. Ktistaki E, Talianidis I (1997) Chicken ovalbumin upstream promoter transcription factors act as auxiliary cofactors for hepatocyte nuclear factor 4 and enhance hepatic gene expression. Mol Cell Biol 17: 2790-2797.

135. Power SC, Cereghini S (1996) Positive regulation of the vHNF1 promoter by the orphan receptors COUP-TF1/Ear3 and COUP-TFII/Arp1. Mol Cell Biol 16: 778-791.

136. Perilhou A, Tourrel-Cuzin C, Zhang P, Kharroubi I, Wang H, et al. (2008) The MODY1 gene for hepatocyte nuclear factor 4alpha and a feedback loop control COUP-TFII expression in pancreatic beta cells. Molecular and cellular biology 28: 4588-4597.

137. Wu Q, Li Y, Liu R, Agadir A, Lee MO, et al. (1997) Modulation of retinoic acid sensitivity in lung cancer cells through dynamic balance of orphan receptors nur77 and COUP-TF and their heterodimerization. The EMBO journal 16: 1656-1669.

138. Rosa A, Brivanlou AH (2011) A regulatory circuitry comprised of miR-302 and the transcription factors OCT4 and NR2F2 regulates human embryonic stem cell differentiation. EMBO J 30: 237-248.

139. Ben-Shushan E, Sharir H, Pikarsky E, Bergman Y (1995) A dynamic balance between ARP-1/COUP-TFII, EAR-3/COUP-TFI, and retinoic acid receptor:retinoid $\mathrm{X}$ receptor heterodimers regulates Oct-3/4 expression in embryonal carcinoma cells. Molecular and cellular biology 15: 1034-1048.

140. Yu RN, Ito M, Jameson JL (1998) The murine Dax-1 promoter is stimulated by SF-1 (steroidogenic factor-1) and inhibited by COUP-TF (chicken ovalbumin upstream promoter-transcription factor) via a composite nuclear receptorregulatory element. Mol Endocrinol 12: 1010-1022.

141. Pineda Torra I, Jamshidi Y, Flavell DM, Fruchart JC, Staels B (2002) Characterization of the human PPARalpha promoter: identification of a functional nuclear receptor response element. Mol Endocrinol 16: 1013-1028.

142. You M, Fischer M, Cho WK, Crabb D (2002) Transcriptional control of the human aldehyde dehydrogenase 2 promoter by hepatocyte nuclear factor 4 : inhibition by cyclic AMP and COUP transcription factors. Archives of biochemistry and biophysics 398: 79-86.

143. Nakshatri H, Chambon $P$ (1994) The directly repeated RG(G/T)TCA motifs of the rat and mouse cellular retinol-binding protein II genes are promiscuous binding sites for RAR, RXR, HNF-4, and ARP-1 homo- and heterodimers. The Journal of biological chemistry 269: 890-902.

144. Rufibach LE, Duncan SA, Battle M, Deeb SS (2006) Transcriptional regulation of the human hepatic lipase (LIPC) gene promoter. J Lipid Res 47: 14631477.

145. Robinson CE, Wu X, Nawaz Z, Onate SA, Gimble JM (1999) A corepressor and chicken ovalbumin upstream promoter transcriptional factor proteins 
modulate peroxisome proliferator-activated receptor-gamma2/retinoid X receptor alpha-activated transcription from the murine lipoprotein lipase promoter. Endocrinology 140: 1586-1593.

146. Jiang G, Nepomuceno L, Hopkins K, Sladek FM (1995) Exclusive homodimerization of the orphan receptor hepatocyte nuclear factor 4 defines a new subclass of nuclear receptors. Mol Cell Biol 15: 5131-5143.

147. Widom RL, Rhee M, Karathanasis SK (1992) Repression by ARP-1 sensitizes apolipoprotein AI gene responsiveness to RXR alpha and retinoic acid. Mol Cell Biol 12: 3380-3389.

148. Sauvaget D, Chauffeton V, Citadelle D, Chatelet FP, Cywiner-Golenzer C, et al. (2002) Restriction of apolipoprotein A-IV gene expression to the intestine villus depends on a hormone-responsive element and parallels differential expression of the hepatic nuclear factor 4alpha and gamma isoforms. J Biol Chem 277: 34540-34548.

149. Ochoa A, Bovard-Houppermans S, Zakin MM (1993) Human apolipoprotein AIV gene expression is modulated by members of the nuclear hormone receptor superfamily. Biochim Biophys Acta 1210: 41-47.

150. Lavrentiadou SN, Hadzopoulou-Cladaras M, Kardassis D, Zannis VI (1999) Binding specificity and modulation of the human ApoCIII promoter activity by heterodimers of ligand-dependent nuclear receptors. Biochemistry 38: 964975.

151. Mietus-Snyder M, Sladek FM, Ginsburg GS, Kuo CF, Ladias JA, et al. (1992) Antagonism between apolipoprotein AI regulatory protein 1, Ear3/COUP-TF, and hepatocyte nuclear factor 4 modulates apolipoprotein CIII gene expression in liver and intestinal cells. Mol Cell Biol 12: 1708-1718.

152. Selva DM, Hogeveen KN, Hammond GL (2005) Repression of the human sex hormone-binding globulin gene in Sertoli cells by upstream stimulatory transcription factors. J Biol Chem 280: 4462-4468.

153. Janne M, Hammond GL (1998) Hepatocyte nuclear factor-4 controls transcription from a TATA-less human sex hormone-binding globulin gene promoter. J Biol Chem 273: 34105-34114.

154. Hammond GL (2011) Diverse roles for sex hormone-binding globulin in reproduction. Biol Reprod 85: 431-441.

155. Kurihara I, Shibata H, Kobayashi S, Suda N, Ikeda Y, et al. (2005) Ubc9 and Protein Inhibitor of Activated STAT 1 Activate Chicken Ovalbumin Upstream Promoter-Transcription Factor I-mediated Human CYP11B2 Gene Transcription. The Journal of biological chemistry 280: 6721-6730.

156. Shibata H, Kobayashi S, Kurihara I, Suda N, Yokota K, et al. (2004) COUP-TF and transcriptional co-regulators in adrenal steroidogenesis. Endocrine research 30: 795-801.

157. Eubank DW, Duplus E, Williams SC, Forest C, Beale EG (2001) Peroxisome proliferator-activated receptor gamma and chicken ovalbumin upstream promoter transcription factor II negatively regulate the phosphoenolpyruvate carboxykinase promoter via a common element. J Biol Chem 276: 3056130569 . 
158. Lee MO, Liu Y, Zhang XK (1995) A retinoic acid response element that overlaps an estrogen response element mediates multihormonal sensitivity in transcriptional activation of the lactoferrin gene. Mol Cell Biol 15: 4194-4207.

159. Liberati C, Cera MR, Secco P, Santoro C, Mantovani R, et al. (2001) Cooperation and competition between the binding of COUP-TFII and NF-Y on human epsilon- and gamma-globin gene promoters. J Biol Chem 276: 41700-41709.

160. Rodriguez JC, Ortiz JA, Hegardt FG, Haro D (1997) Chicken ovalbumin upstream-promoter transcription factor (COUP-TF) could act as a transcriptional activator or repressor of the mitochondrial 3-hydroxy-3methylglutaryl-CoA synthase gene. Biochem J 326 ( Pt 2): 587-592.

161. Kyrmizi I, Hatzis P, Katrakili N, Tronche F, Gonzalez FJ, et al. (2006) Plasticity and expanding complexity of the hepatic transcription factor network during liver development. Genes Dev 20: 2293-2305.

162. Tanimoto K, Liu Q, Grosveld F, Bungert J, Engel JD (2000) Context-dependent EKLF responsiveness defines the developmental specificity of the human epsilon-globin gene in erythroid cells of YAC transgenic mice. Genes Dev 14: 2778-2794.

163. Kang J, Yoo J, Lee S, Tang W, Aguilar B, et al. (2010) An exquisite crosscontrol mechanism among endothelial cell fate regulators directs the plasticity and heterogeneity of lymphatic endothelial cells. Blood 116: 140-150.

164. Huggins GS, Bacani CJ, Boltax J, Aikawa R, Leiden JM (2001) Friend of GATA 2 physically interacts with chicken ovalbumin upstream promoter-TF2 (COUP-TF2) and COUP-TF3 and represses COUP-TF2-dependent activation of the atrial natriuretic factor promoter. J Biol Chem 276: 28029-28036.

165. Li X, Misik AJ, Rieder CV, Solaro RJ, Lowen A, et al. (2002) Thyroid hormone receptor alpha 1 regulates expression of the $\mathrm{Na}+\mathrm{H}+$ exchanger $(\mathrm{NHE} 1)$. J Biol Chem 277: 28656-28662.

166. Fernandez-Rachubinski F, Fliegel L (2001) COUP-TFI and COUP-TFII regulate expression of the NHE through a nuclear hormone responsive element with enhancer activity. Eur J Biochem 268: 620-634.

167. Nakshatri H, Mendonca MS, Bhat-Nakshatri P, Patel NM, Goulet RJ, Jr., et al. (2000) The orphan receptor COUP-TFII regulates G2/M progression of breast cancer cells by modulating the expression/activity of p21(WAF1/CIP1), cyclin D1, and cdk2. Biochemical and biophysical research communications 270: 1144-1153.

168. Suzuki T, Moriya T, Darnel AD, Takeyama J, Sasano H (2000) Immunohistochemical distribution of chicken ovalbumin upstream promoter transcription factor II in human tissues. Mol Cell Endocrinol 164: 69-75.

169. Ferguson RD, Novosyadlyy R, Fierz Y, Alikhani N, Sun H, et al. (2012) Hyperinsulinemia enhances c-Myc-mediated mammary tumor development and advances metastatic progression to the lung in a mouse model of type 2 diabetes. Breast Cancer Res 14: R8.

170. Gunter MJ, Hoover DR, Yu H, Wassertheil-Smoller S, Rohan TE, et al. (2009) Insulin, insulin-like growth factor-I, and risk of breast cancer in postmenopausal women. J Natl Cancer Inst 101: 48-60. 
171. Perilhou A, Tourrel-Cuzin C, Kharroubi I, Henique C, Fauveau V, et al. (2008) The transcription factor COUP-TFII is negatively regulated by insulin and glucose via Foxo1- and ChREBP-controlled pathways. Molecular and cellular biology 28: 6568-6579.

172. Deaton AM, Bird A (2011) CpG islands and the regulation of transcription. Genes Dev 25: 1010-1022.

173. Shenker N, Flanagan JM (2012) Intragenic DNA methylation: implications of this epigenetic mechanism for cancer research. Br J Cancer 106: 248-253.

174. Enjuanes A, Fernandez V, Hernandez L, Navarro A, Bea S, et al. (2011) Identification of methylated genes associated with aggressive clinicopathological features in mantle cell lymphoma. PLoS One 6: e19736.

175. Bullinger L, Ehrich M, Dohner K, Schlenk RF, Dohner H, et al. (2010) Quantitative DNA methylation predicts survival in adult acute myeloid leukemia. Blood 115: 636-642.

176. Bell A, Bell D, Weber RS, El-Naggar AK (2011) CpG island methylation profiling in human salivary gland adenoid cystic carcinoma. Cancer 117: 2898-2909.

177. Vincent A, Omura N, Hong SM, Jaffe A, Eshleman J, et al. (2011) Genomewide analysis of promoter methylation associated with gene expression profile in pancreatic adenocarcinoma. Clinical cancer research : an official journal of the American Association for Cancer Research 17: 4341-4354.

178. Tommasi S, Karm DL, Wu X, Yen Y, Pfeifer GP (2009) Methylation of homeobox genes is a frequent and early epigenetic event in breast cancer. Breast Cancer Res 11: R14.

179. Irizarry RA, Ladd-Acosta C, Wen B, Wu Z, Montano C, et al. (2009) The human colon cancer methylome shows similar hypo- and hypermethylation at conserved tissue-specific CpG island shores. Nat Genet 41: 178-186.

180. Holbeck S, Chang J, Best AM, Bookout AL, Mangelsdorf DJ, et al. (2010) Expression profiling of nuclear receptors in the NCI60 cancer cell panel reveals receptor-drug and receptor-gene interactions. Molecular endocrinology 24: 1287-1296.

181. Petit FG, Salas R, Tsai MJ, Tsai SY (2004) The regulation of COUP-TFII gene expression by Ets-1 is enhanced by the steroid receptor co-activators. Mechanisms of ageing and development 125: 719-732.

182. Ma X, Xu L, Wang S, Cui B, Li X, et al. (2011) Deletion of steroid receptor coactivator-3 gene ameliorates hepatic steatosis. Journal of hepatology 55: 445-452.

183. More E, Fellner T, Doppelmayr H, Hauser-Kronberger C, Dandachi N, et al. (2003) Activation of the MAP kinase pathway induces chicken ovalbumin upstream promoter-transcription factor II (COUP-TFII) expression in human breast cancer cell lines. J Endocrinol 176: 83-94.

184. Aerbajinai W, Zhu J, Kumkhaek C, Chin K, Rodgers GP (2009) SCF induces gamma-globin gene expression by regulating downstream transcription factor COUP-TFII. Blood 114: 187-194. 
185. Krishnan V, Pereira FA, Qiu Y, Chen CH, Beachy PA, et al. (1997) Mediation of Sonic hedgehog-induced expression of COUP-TFII by a protein phosphatase. Science 278: 1947-1950.

186. Ni YG, Wang N, Cao DJ, Sachan N, Morris DJ, et al. (2007) FoxO transcription factors activate Akt and attenuate insulin signaling in heart by inhibiting protein phosphatases. Proc Natl Acad Sci U S A 104: 20517-20522.

187. Garcia A, Kandel JJ (2012) Notch: a key regulator of tumor angiogenesis and metastasis. Histol Histopathol 27: 151-156.

188. Gu JW, Rizzo P, Pannuti A, Golde T, Osborne B, et al. (2012) Notch signals in the endothelium and cancer "stem-like" cells: opportunities for cancer therapy. Vasc Cell 4: 7.

189. Leong KG, Niessen K, Kulic I, Raouf A, Eaves C, et al. (2007) Jagged1mediated Notch activation induces epithelial-to-mesenchymal transition through Slug-induced repression of E-cadherin. J Exp Med 204: 2935-2948.

190. Magnifico A, Albano L, Campaner S, Delia D, Castiglioni F, et al. (2009) Tumor-initiating cells of HER2-positive carcinoma cell lines express the highest oncoprotein levels and are sensitive to trastuzumab. Clin Cancer Res 15: 2010-2021.

191. Pannuti A, Foreman K, Rizzo P, Osipo C, Golde T, et al. (2010) Targeting Notch to target cancer stem cells. Clin Cancer Res 16: 3141-3152.

192. Harrison H, Farnie G, Howell SJ, Rock RE, Stylianou S, et al. (2010) Regulation of breast cancer stem cell activity by signaling through the Notch4 receptor. Cancer Res 70: 709-718.

193. Stylianou S, Clarke RB, Brennan K (2006) Aberrant activation of notch signaling in human breast cancer. Cancer Res 66: 1517-1525.

194. Srinivasan RS, Geng X, Yang Y, Wang Y, Mukatira S, et al. (2010) The nuclear hormone receptor Coup-TFII is required for the initiation and early maintenance of Prox 1 expression in lymphatic endothelial cells. Genes Dev 24: 696-707.

195. You LR, Lin FJ, Lee CT, DeMayo FJ, Tsai MJ, et al. (2005) Suppression of Notch signalling by the COUP-TFII transcription factor regulates vein identity. Nature 435: 98-104.

196. Francois M, Harvey NL, Hogan BM (2011) The transcriptional control of lymphatic vascular development. Physiology (Bethesda) 26: 146-155.

197. Calonge MJ, Seoane J, Massague J (2004) Opposite Smad and chicken ovalbumin upstream promoter transcription factor inputs in the regulation of the collagen VII gene promoter by transforming growth factor-beta. J Biol Chem 279: 23759-23765.

198. Vittet D, Merdzhanova G, Prandini MH, Feige JJ, Bailly S (2012) TGFss1 inhibits lymphatic endothelial cell differentiation from mouse embryonic stem cells. J Cell Physiol.

199. Incassati A, Chandramouli A, Eelkema R, Cowin P (2010) Key signaling nodes in mammary gland development and cancer: beta-catenin. Breast Cancer Res 12: 213.

200. Bardoux P, Zhang P, Flamez D, Perilhou A, Lavin TA, et al. (2005) Essential role of chicken ovalbumin upstream promoter-transcription factor II in insulin 
secretion and insulin sensitivity revealed by conditional gene knockout. Diabetes 54: 1357-1363.

201. Boutant M, Ramos OH, Tourrel-Cuzin C, Movassat J, Ilias A, et al. (2012) COUP-TFII controls mouse pancreatic beta-cell mass through GLP-1-betacatenin signaling pathways. PLoS One 7: e30847.

202. Hanahan D, Weinberg RA (2011) Hallmarks of cancer: the next generation. Cell 144: 646-674.

203. Lin FJ, Chen X, Qin J, Hong YK, Tsai MJ, et al. (2010) Direct transcriptional regulation of neuropilin-2 by COUP-TFII modulates multiple steps in murine lymphatic vessel development. The Journal of clinical investigation 120: 1694-1707.

204. Hoeben A, Landuyt B, Highley MS, Wildiers H, Van Oosterom AT, et al. (2004) Vascular endothelial growth factor and angiogenesis. Pharmacol Rev 56: 549580.

205. Qin J, Chen X, Yu-Lee LY, Tsai MJ, Tsai SY (2010) Nuclear receptor COUPTFII controls pancreatic islet tumor angiogenesis by regulating vascular endothelial growth factor/vascular endothelial growth factor receptor-2 signaling. Cancer research 70: 8812-8821.

206. Qin J, Chen X, Xie X, Tsai MJ, Tsai SY (2010) COUP-TFII regulates tumor growth and metastasis by modulating tumor angiogenesis. Proceedings of the National Academy of Sciences of the United States of America 107: 36873692.

207. Achen MG, McColl BK, Stacker SA (2005) Focus on lymphangiogenesis in tumor metastasis. Cancer Cell 7: 121-127.

208. Tobler NE, Detmar M (2006) Tumor and lymph node lymphangiogenesis-impact on cancer metastasis. J Leukoc Biol 80: 691-696.

209. Nagasaki S, Suzuki T, Miki Y, Akahira J, Shibata H, et al. (2009) Chicken ovalbumin upstream promoter transcription factor II in human breast carcinoma: possible regulator of lymphangiogenesis via vascular endothelial growth factor-C expression. Cancer science 100: 639-645.

210. Prahalad P, Dakshanamurthy S, Ressom H, Byers SW (2010) Retinoic acid mediates regulation of network formation by COUP-TFII and VE-cadherin expression by TGFbeta receptor kinase in breast cancer cells. PLoS One 5: e10023.

211. Navab R, Gonzalez-Santos JM, Johnston MR, Liu J, Brodt P, et al. (2004) Expression of chicken ovalbumin upstream promoter-transcription factor II enhances invasiveness of human lung carcinoma cells. Cancer research 64: 5097-5105.

212. Annecke K, Schmitt M, Euler U, Zerm M, Paepke D, et al. (2008) uPA and PAI1 in breast cancer: review of their clinical utility and current validation in the prospective NNBC-3 trial. Adv Clin Chem 45: 31-45.

213. Harbeck N, Kates RE, Schmitt M, Gauger K, Kiechle M, et al. (2004) Urokinase-type plasminogen activator and its inhibitor type 1 predict disease outcome and therapy response in primary breast cancer. Clin Breast Cancer 5: 348-352. 
214. Shimizu M, Cohen B, Goldvasser P, Berman H, Virtanen C, et al. (2011) Plasminogen activator uPA is a direct transcriptional target of the JAG1Notch receptor signaling pathway in breast cancer. Cancer Res 71: 277-286.

215. Suzuki T, Takahashi K, Darnel AD, MoriyaT, Murakami O, et al. (2000) Chicken ovalbumin upstream promoter transcription factor II in the human adrenal cortex and its disorders. J Clin Endocrinol Metab 85: 2752-2757.

216. van den Driesche S, Walker M, McKinnell C, Scott HM, Eddie SL, et al. (2012) Proposed Role for COUP-TFII in Regulating Fetal Leydig Cell Steroidogenesis, Perturbation of Which Leads to Masculinization Disorders in Rodents. PLoS One 7: e37064.

217. Bakke M, Lund J (1995) Mutually exclusive interactions of two nuclear orphan receptors determine activity of a cyclic adenosine 3',5'-monophosphateresponsive sequence in the bovine CYP17 gene. Mol Endocrinol 9: 327-339.

218. Buholzer CF, Arrighi JF, Abraham S, Piguet V, Capponi AM, et al. (2005) Chicken ovalbumin upstream promoter-transcription factor is a negative regulator of steroidogenesis in bovine adrenal glomerulosa cells. Mol Endocrinol 19: 65-75.

219. Zeitoun K, Takayama K, Michael MD, Bulun SE (1999) Stimulation of aromatase $\mathrm{P} 450$ promoter (II) activity in endometriosis and its inhibition in endometrium are regulated by competitive binding of steroidogenic factor-1 and chicken ovalbumin upstream promoter transcription factor to the same cis-acting element. Molecular endocrinology 13: 239-253.

220. Yang C, Yu B, Zhou D, Chen S (2002) Regulation of aromatase promoter activity in human breast tissue by nuclear receptors. Oncogene 21: 2854-2863.

221. Chen S, Ye J, Kijima I, Kinoshita Y, Zhou D (2005) Positive and negative transcriptional regulation of aromatase expression in human breast cancer tissue. J Steroid Biochem Mol Biol 95: 17-23.

222. Sikora MJ, Cordero KE, Larios JM, Johnson MD, Lippman ME, et al. (2009) The androgen metabolite 5alpha-androstane-3beta,17beta-diol (3betaAdiol) induces breast cancer growth via estrogen receptor: implications for aromatase inhibitor resistance. Breast Cancer Res Treat 115: 289-296.

223. Sikora MJ, Strumba V, Lippman ME, Johnson MD, Rae JM (2012) Mechanisms of estrogen-independent breast cancer growth driven by low estrogen concentrations are unique versus complete estrogen deprivation. Breast Cancer Res Treat.

224. Bulun SE, Chen D, Moy I, Brooks DC, Zhao H (2012) Aromatase, breast cancer and obesity: a complex interaction. Trends Endocrinol Metab 23: 83-89.

225. Marquez-Garban DC, Chen HW, Goodglick L, Fishbein MC, Pietras RJ (2009) Targeting aromatase and estrogen signaling in human non-small cell lung cancer. Ann N Y Acad Sci 1155: 194-205.

226. Litchfield LM, Klinge CM (2012) Multiple roles of COUP-TFII in cancer initiation and progression. Journal of Molecular Endocrinology.

227. Qiu Y, Pereira FA, DeMayo FJ, Lydon JP, Tsai SY, et al. (1997) Null mutation of mCOUP-TFI results in defects in morphogenesis of the glossopharyngeal ganglion, axonal projection, and arborization. Genes Dev 11: 1925-1937. 
228. Sjoblom T, Jones S, Wood LD, Parsons DW, Lin J, et al. (2006) The consensus coding sequences of human breast and colorectal cancers. Science 314: 268274.

229. Almendro V, Fuster G (2011) Heterogeneity of breast cancer: etiology and clinical relevance. Clin Transl Oncol 13: 767-773.

230. Huang TH, Esteller M (2010) Chromatin remodeling in mammary gland differentiation and breast tumorigenesis. Cold Spring Harb Perspect Biol 2: a004515.

231. Russo J, Russo IH (2006) The role of estrogen in the initiation of breast cancer. J Steroid Biochem Mol Biol 102: 89-96.

232. Jensen EV, Cheng G, Palmieri C, Saji S, Makela S, et al. (2001) Estrogen receptors and proliferation markers in primary and recurrent breast cancer. Proc Natl Acad Sci U S A 98: 15197-15202.

233. Wakeling AE, Bowler J (1992) ICI 182,780, a new antioestrogen with clinical potential. J Steroid Biochem Mol Biol 43: 173-177.

234. Santen RJ, Brodie H, Simpson ER, Siiteri PK, Brodie A (2009) History of aromatase: saga of an important biological mediator and therapeutic target. Endocr Rev 30: 343-375.

235. Rohr O, Aunis D, Schaeffer E (1997) COUP-TF and Sp1 interact and cooperate in the transcriptional activation of the human immunodeficiency virus type 1 long terminal repeat in human microglial cells. J Biol Chem 272: 3114931155.

236. Rohr O, Schwartz C, Hery C, Aunis D, Tardieu M, et al. (2000) The nuclear receptor chicken ovalbumin upstream promoter transcription factor interacts with HIV-1 Tat and stimulates viral replication in human microglial cells. J Biol Chem 275: 2654-2660.

237. Senawong T, Peterson VJ, Avram D, Shepherd DM, Frye RA, et al. (2003) Involvement of the histone deacetylase SIRT1 in chicken ovalbumin upstream promoter transcription factor (COUP-TF)-interacting protein 2-mediated transcriptional repression. The Journal of biological chemistry 278: 4304143050.

238. Klinge CM (1999) Role of estrogen receptor ligand and estrogen response element sequence on interaction with chicken ovalbumin upstream promoter transcription factor (COUP-TF). J Steroid Biochem Mol Biol 71: 1-19.

239. Klinge CM, Kaur K, Swanson HI (2000) The aryl hydrocarbon receptor interacts with estrogen receptor alpha and orphan receptors COUP-TFI and ERRalpha1. Arch Biochem Biophys 373: 163-174.

240. Keen JC, Davidson NE (2003) The biology of breast carcinoma. Cancer 97: 825833.

241. Yang Q, Sakurai T, Kakudo K (2002) Retinoid, retinoic acid receptor beta and breast cancer. Breast Cancer Res Treat 76: 167-173.

242. Girvan AC, Teng Y, Casson LK, Thomas SD, Juliger S, et al. (2006) AGRO100 inhibits activation of nuclear factor-kappaB (NF-kappaB) by forming a complex with NF-kappaB essential modulator (NEMO) and nucleolin. Mol Cancer Ther 5: 1790-1799. 
243. Hanakahi LA, Bu Z, Maizels N (2000) The C-terminal domain of nucleolin accelerates nucleic acid annealing. Biochemistry 39: 15493-15499.

244. Qin L, Liao L, Redmond A, Young L, Yuan Y, et al. (2008) The AIB1 oncogene promotes breast cancer metastasis by activation of PEA3-mediated matrix metalloproteinase 2 (MMP2) and MMP9 expression. Molecular and cellular biology 28: 5937-5950.

245. Greene FL, Sobin LH (2008) The staging of cancer: a retrospective and prospective appraisal. CA Cancer J Clin 58: 180-190.

246. Qiu Y, Krishnan V, Zeng Z, Gilbert DJ, Copeland NG, et al. (1995) Isolation, characterization, and chromosomal localization of mouse and human COUPTF I and II genes. Genomics 29: 240-246.

247. Teng Y, Girvan AC, Casson LK, Pierce WM, Jr., Qian M, et al. (2007) AS1411 alters the localization of a complex containing protein arginine methyltransferase 5 and nucleolin. Cancer Res 67: 10491-10500.

248. Desai D, Michalak M, Singh NK, Niles RM (1996) Inhibition of retinoic acid receptor function and retinoic acid-regulated gene expression in mouse melanoma cells by calreticulin. A potential pathway for cyclic AMP regulation of retinoid action. J Biol Chem 271: 15153-15159.

249. Liang ZD, Lippman SM, Wu TT, Lotan R, Xu XC (2006) RRIG1 mediates effects of retinoic acid receptor beta2 on tumor cell growth and gene expression through binding to and inhibition of RhoA. Cancer research 66: 7111-7118.

250. Mattingly KA, Ivanova MM, Riggs KA, Wickramasinghe NS, Barch MJ, et al. (2008) Estradiol stimulates transcription of nuclear respiratory factor-1 and increases mitochondrial biogenesis. Mol Endocrinol 22: 609-622.

251. Reyes-Reyes EM, Teng Y, Bates PJ (2010) A new paradigm for aptamer therapeutic AS1411 action: uptake by macropinocytosis and its stimulation by a nucleolin-dependent mechanism. Cancer Res 70: 8617-8629.

252. Bates PJ, Laber DA, Miller DM, Thomas SD, Trent JO (2009) Discovery and development of the G-rich oligonucleotide AS1411 as a novel treatment for cancer. Exp Mol Pathol 86: 151-164.

253. Mongelard F, Bouvet P (2007) Nucleolin: a multiFACeTed protein. Trends Cell Biol 17: 80-86.

254. Tediose T, Kolev M, Sivasankar B, Brennan P, Morgan BP, et al. (2010) Interplay between REST and nucleolin transcription factors: a key mechanism in the overexpression of genes upon increased phosphorylation. Nucleic acids research 38: 2799-2812.

255. Ying GG, Proost P, van Damme J, Bruschi M, Introna M, et al. (2000) Nucleolin, a novel partner for the Myb transcription factor family that regulates their activity. J Biol Chem 275: 4152-4158.

256. Tsou JH, Chang KY, Wang WC, Tseng JT, Su WC, et al. (2008) Nucleolin regulates c-Jun/Sp1-dependent transcriptional activation of cPLA2alpha in phorbol ester-treated non-small cell lung cancer A549 cells. Nucleic Acids Res 36: 217-227.

257. Uribe DJ, Guo K, Shin YJ, Sun D (2011) Heterogeneous nuclear ribonucleoprotein $\mathrm{K}$ and nucleolin as transcriptional activators of the vascular 
endothelial growth factor promoter through interaction with secondary DNA structures. Biochemistry 50: 3796-3806.

258. Berns EM, van Staveren IL, Klijn JG, Foekens JA (1998) Predictive value of SRC-1 for tamoxifen response of recurrent breast cancer. Breast Cancer Res Treat 48: 87-92.

259. McBryan J, Theissen SM, Byrne C, Hughes E, Cocchiglia S, et al. (2012) Metastatic progression with resistance to aromatase inhibitors is driven by the steroid receptor coactivator SRC-1. Cancer Res 72: 548-559.

260. Xie X, Qin J, Lin SH, Tsai SY, Tsai MJ (2011) Nuclear receptor chicken ovalbumin upstream promoter-transcription factor II (COUP-TFII) modulates mesenchymal cell commitment and differentiation. Proceedings of the National Academy of Sciences of the United States of America 108: 1484314848.

261. Bates PJ, Kahlon JB, Thomas SD, Trent JO, Miller DM (1999) Antiproliferative activity of G-rich oligonucleotides correlates with protein binding. J Biol Chem 274: 26369-26377.

262. Rousseau C, Pettersson F, Couture MC, Paquin A, Galipeau J, et al. (2003) The $\mathrm{N}$-terminal of the estrogen receptor (ERalpha) mediates transcriptional crosstalk with the retinoic acid receptor in human breast cancer cells. J Steroid Biochem Mol Biol 86: 1-14.

263. Xu XC (2007) Tumor-suppressive activity of retinoic acid receptor-beta in cancer. Cancer Lett 253: 14-24.

264. Masiuk M, Urasinska E, Domagala W (2004) Simultaneous measurement of nucleolin and estrogen receptor in breast cancer cells by laser scanning cytometry. Anticancer Res 24: 963-966.

265. Storck S, Shukla M, Dimitrov S, Bouvet P (2007) Functions of the histone chaperone nucleolin in diseases. Subcell Biochem 41: 125-144.

266. van de Vijver MJ, He YD, van't Veer LJ, Dai H, Hart AA, et al. (2002) A geneexpression signature as a predictor of survival in breast cancer. N Engl J Med 347: 1999-2009.

267. Conzen SD (2008) Minireview: nuclear receptors and breast cancer. Mol Endocrinol 22: 2215-2228.

268. Mi Y, Thomas SD, Xu X, Casson LK, Miller DM, et al. (2003) Apoptosis in leukemia cells is accompanied by alterations in the levels and localization of nucleolin. J Biol Chem 278: 8572-8579.

269. Kraus WL (2008) Transcriptional control by PARP-1: chromatin modulation, enhancer-binding, coregulation, and insulation. Curr Opin Cell Biol 20: 294302.

270. Ju BG, Lunyak VV, Perissi V, Garcia-Bassets I, Rose DW, et al. (2006) A topoisomerase IIbeta-mediated dsDNA break required for regulated transcription. Science 312: 1798-1802.

271. Malanga M, Czubaty A, Girstun A, Staron K, Althaus FR (2008) Poly(ADPribose) binds to the splicing factor ASF/SF2 and regulates its phosphorylation by DNA topoisomerase I. J Biol Chem 283: 19991-19998. 
272. Gonzalez V, Guo K, Hurley L, Sun D (2009) Identification and characterization of nucleolin as a c-myc G-quadruplex-binding protein. J Biol Chem 284: 23622-23635.

273. Soundararajan S, Chen W, Spicer EK, Courtenay-Luck N, Fernandes DJ (2008) The nucleolin targeting aptamer AS1411 destabilizes Bcl-2 messenger RNA in human breast cancer cells. Cancer Res 68: 2358-2365.

274. Ishimaru D, Zuraw L, Ramalingam S, Sengupta TK, Bandyopadhyay S, et al. (2010) Mechanism of regulation of bcl-2 mRNA by nucleolin and A+U-rich element-binding factor 1 (AUF1). J Biol Chem 285: 27182-27191.

275. Theodosiou M, Laudet V, Schubert M (2010) From carrot to clinic: an overview of the retinoic acid signaling pathway. Cell Mol Life Sci 67: 1423-1445.

276. Faria TN, Mendelsohn C, Chambon P, Gudas LJ (1999) The targeted disruption of both alleles of RARbeta(2) in F9 cells results in the loss of retinoic acidassociated growth arrest. J Biol Chem 274: 26783-26788.

277. Tamimi RM, Colditz GA, Hankinson SE (2009) Circulating carotenoids, mammographic density, and subsequent risk of breast cancer. Cancer Res 69: 9323-9329.

278. Wijayaratne AL, Nagel SC, Paige LA, Christensen DJ, Norris JD, et al. (1999) Comparative analyses of mechanistic differences among antiestrogens. Endocrinology 140: 5828-5840.

279. (2005) Effects of chemotherapy and hormonal therapy for early breast cancer on recurrence and 15-year survival: an overview of the randomised trials. Lancet 365: 1687-1717.

280. Gu Z, Lee RY, Skaar TC, Bouker KB, Welch JN, et al. (2002) Association of interferon regulatory factor-1, nucleophosmin, nuclear factor-kappaB, and cyclic AMP response element binding with acquired resistance to Faslodex (ICI 182,780). Cancer Res 62: 3428-3437.

281. Perkins ND (2007) Integrating cell-signalling pathways with NF-kappaB and IKK function. Nat Rev Mol Cell Biol 8: 49-62.

282. Cogswell PC, Guttridge DC, Funkhouser WK, Baldwin AS, Jr. (2000) Selective activation of NF-kappa B subunits in human breast cancer: potential roles for NF-kappa B2/p52 and for Bcl-3. Oncogene 19: 1123-1131.

283. Zhou Y, Eppenberger-Castori S, Marx C, Yau C, Scott GK, et al. (2005) Activation of nuclear factor-kappaB (NFkappaB) identifies a high-risk subset of hormone-dependent breast cancers. Int J Biochem Cell Biol 37: 1130-1144.

284. Zhou Y, Yau C, Gray JW, Chew K, Dairkee SH, et al. (2007) Enhanced NF kappa B and AP-1 transcriptional activity associated with antiestrogen resistant breast cancer. BMC Cancer 7: 59.

285. Brunner N, Boysen B, Jirus S, Skaar TC, Holst-Hansen C, et al. (1997) MCF7/LCC9: an antiestrogen-resistant MCF-7 variant in which acquired resistance to the steroidal antiestrogen ICI 182,780 confers an early crossresistance to the nonsteroidal antiestrogen tamoxifen. Cancer Res 57: 34863493.

286. Dell H, Hadzopoulou-Cladaras M (1999) CREB-binding protein is a transcriptional coactivator for hepatocyte nuclear factor-4 and enhances apolipoprotein gene expression. J Biol Chem 274: 9013-9021. 
287. Lanz RB, McKenna NJ, Onate SA, Albrecht U, Wong J, et al. (1999) A steroid receptor coactivator, SRA, functions as an RNA and is present in an SRC-1 complex. Cell 97: 17-27.

288. Koh SS, Chen D, Lee YH, Stallcup MR (2001) Synergistic enhancement of nuclear receptor function by 160 coactivators and two coactivators with protein methyltransferase activities. J Biol Chem 276: 1089-1098.

289. Yun JJ, Tsao MS, Der SD (2011) Differential utilization of NF-kappaB RELA and RELB in response to extracellular versus intracellular polyIC stimulation in HT1080 cells. BMC immunology 12: 15.

290. Panguluri SK, Bhatnagar S, Kumar A, McCarthy JJ, Srivastava AK, et al. (2010) Genomic profiling of messenger RNAs and microRNAs reveals potential mechanisms of TWEAK-induced skeletal muscle wasting in mice. PLoS One 5: e8760.

291. Sansone P, Storci G, Tavolari S, Guarnieri T, Giovannini C, et al. (2007) IL-6 triggers malignant features in mammospheres from human ductal breast carcinoma and normal mammary gland. The Journal of clinical investigation 117: 3988-4002.

292. Vendrell JA, Ghayad S, Ben-Larbi S, Dumontet C, Mechti N, et al. (2007) A20/TNFAIP3, a new estrogen-regulated gene that confers tamoxifen resistance in breast cancer cells. Oncogene 26: 4656-4667.

293. Silverman MD, Zamora DO, Pan Y, Texeira PV, Planck SR, et al. (2001) Cell adhesion molecule expression in cultured human iris endothelial cells. Investigative ophthalmology \& visual science 42: 2861-2866.

294. Qian BZ, Li J, Zhang H, Kitamura T, Zhang J, et al. (2011) CCL2 recruits inflammatory monocytes to facilitate breast-tumour metastasis. Nature 475: 222-225.

295. Song CH, Lee HJ, Park E, Lee K (2012) The chicken ovalbumin upstream promoter-transcription factor II negatively regulates the transactivation of androgen receptor in prostate cancer cells. PLoS One 7: e49026.

296. Gao Z, Chiao P, Zhang X, Zhang X, Lazar MA, et al. (2005) Coactivators and corepressors of NF-kappaB in IkappaB alpha gene promoter. J Biol Chem 280: 21091-21098.

297. Burke JR, Pattoli MA, Gregor KR, Brassil PJ, MacMaster JF, et al. (2003) BMS345541 is a highly selective inhibitor of I kappa B kinase that binds at an allosteric site of the enzyme and blocks NF-kappa B-dependent transcription in mice. J Biol Chem 278: 1450-1456.

298. Opipari AW, Jr., Hu HM, Yabkowitz R, Dixit VM (1992) The A20 zinc finger protein protects cells from tumor necrosis factor cytotoxicity. J Biol Chem 267: 12424-12427.

299. Soria G, Ben-Baruch A (2008) The inflammatory chemokines CCL2 and CCL5 in breast cancer. Cancer letters 267: 271-285.

300. Huber MA, Azoitei N, Baumann B, Grunert S, Sommer A, et al. (2004) NFkappaB is essential for epithelial-mesenchymal transition and metastasis in a model of breast cancer progression. J Clin Invest 114: 569-581. 
301. Sovak MA, Bellas RE, Kim DW, Zanieski GJ, Rogers AE, et al. (1997) Aberrant nuclear factor-kappaB/Rel expression and the pathogenesis of breast cancer. The Journal of clinical investigation 100: 2952-2960.

302. Knupfer H, Preiss R (2007) Significance of interleukin-6 (IL-6) in breast cancer (review). Breast cancer research and treatment 102: 129-135.

303. Korkaya H, Kim GI, Davis A, Malik F, Henry NL, et al. (2012) Activation of an IL6 inflammatory loop mediates trastuzumab resistance in HER2+ breast cancer by expanding the cancer stem cell population. Mol Cell 47: 570-584.

304. Rosette C, Roth RB, Oeth P, Braun A, Kammerer S, et al. (2005) Role of ICAM1 in invasion of human breast cancer cells. Carcinogenesis 26: 943-950.

305. Krikos A, Laherty CD, Dixit VM (1992) Transcriptional activation of the tumor necrosis factor alpha-inducible zinc finger protein, A20, is mediated by kappa B elements. J Biol Chem 267: 17971-17976.

306. Bren GD, Solan NJ, Miyoshi H, Pennington KN, Pobst LJ, et al. (2001) Transcription of the RelB gene is regulated by NF-kappaB. Oncogene 20: 7722-7733.

307. Lombardi L, Ciana P, Cappellini C, Trecca D, Guerrini L, et al. (1995) Structural and functional characterization of the promoter regions of the NFKB2 gene. Nucleic Acids Res 23: 2328-2336.

308. Ten RM, Paya CV, Israel N, Le Bail O, Mattei MG, et al. (1992) The characterization of the promoter of the gene encoding the p50 subunit of NFkappa B indicates that it participates in its own regulation. EMBO J 11: 195203.

309. Hannink M, Temin HM (1990) Structure and autoregulation of the c-rel promoter. Oncogene 5: 1843-1850.

310. Lerebours F, Vacher S, Andrieu C, Espie M, Marty M, et al. (2008) NF-kappa B genes have a major role in inflammatory breast cancer. BMC Cancer 8: 41.

311. Zhang X, Wang JM, Gong WH, Mukaida N, Young HA (2001) Differential regulation of chemokine gene expression by 15 -deoxy-delta 12,14 prostaglandin J2. J Immunol 166: 7104-7111.

312. Singh S, Aggarwal BB (1995) Activation of transcription factor NF-kappa B is suppressed by curcumin (diferuloylmethane) [corrected]. J Biol Chem 270: 24995-25000.

313. Jobin C, Bradham CA, Russo MP, Juma B, Narula AS, et al. (1999) Curcumin blocks cytokine-mediated NF-kappa B activation and proinflammatory gene expression by inhibiting inhibitory factor I-kappa B kinase activity. J Immunol 163: 3474-3483.

314. Edderkaoui M, Odinokova I, Ohno I, Gukovsky I, Go VL, et al. (2008) Ellagic acid induces apoptosis through inhibition of nuclear factor kappa B in pancreatic cancer cells. World J Gastroenterol 14: 3672-3680.

315. Pflieger D, Gonnet F, de la Fuente van Bentem S, Hirt H, de la Fuente A (2011) Linking the proteins--elucidation of proteome-scale networks using mass spectrometry. Mass Spectrom Rev 30: 268-297.

316. Pieroni E, de la Fuente van Bentem S, Mancosu G, Capobianco E, Hirt H, et al. (2008) Protein networking: insights into global functional organization of proteomes. Proteomics 8: 799-816. 
317. Lonard DM, O'Malley BW (2012) Nuclear receptor coregulators: modulators of pathology and therapeutic targets. Nat Rev Endocrinol 8: 598-604.

318. Vargas-Roig LM, Gago FE, Tello O, Aznar JC, Ciocca DR (1998) Heat shock protein expression and drug resistance in breast cancer patients treated with induction chemotherapy. Int J Cancer 79: 468-475.

319. Rust W, Kingsley K, Petnicki T, Padmanabhan S, Carper SW, et al. (1999) Heat shock protein 27 plays two distinct roles in controlling human breast cancer cell migration on laminin-5. Mol Cell Biol Res Commun 1: 196-202.

320. Ciocca DR, Green S, Elledge RM, Clark GM, Pugh R, et al. (1998) Heat shock proteins hsp27 and hsp70: lack of correlation with response to tamoxifen and clinical course of disease in estrogen receptor-positive metastatic breast cancer (a Southwest Oncology Group Study). Clinical cancer research : an official journal of the American Association for Cancer Research 4: 12631266.

321. Cano A, Coffer AI, Adatia R, Millis RR, Rubens RD, et al. (1986) Histochemical studies with an estrogen receptor-related protein in human breast tumors. Cancer Res 46: 6475-6480.

322. Kang SH, Kang KW, Kim KH, Kwon B, Kim SK, et al. (2008) Upregulated HSP27 in human breast cancer cells reduces Herceptin susceptibility by increasing Her2 protein stability. BMC Cancer 8: 286.

323. Casado P, Zuazua-Villar P, del Valle E, Martinez-Campa C, Lazo PS, et al. (2007) Vincristine regulates the phosphorylation of the antiapoptotic protein HSP27 in breast cancer cells. Cancer Lett 247: 273-282.

324. Kanagasabai R, Krishnamurthy K, Druhan LJ, Ilangovan G (2011) Forced expression of heat shock protein 27 (Hsp27) reverses P-glycoprotein (ABCB1)-mediated drug efflux and MDR1 gene expression in Adriamycinresistant human breast cancer cells. J Biol Chem 286: 33289-33300.

325. Horman S, Galand P, Mosselmans R, Legros N, Leclercq G, et al. (1997) Changes in the phosphorylation status of the $27 \mathrm{kDa}$ heat shock protein (HSP27) associated with the modulation of growth and/or differentiation in MCF-7 cells. Cell Prolif 30: 21-35.

326. Brunner N, Frandsen TL, Holst-Hansen C, Bei M, Thompson EW, et al. (1993) MCF7/LCC2: a 4-hydroxytamoxifen resistant human breast cancer variant that retains sensitivity to the steroidal antiestrogen ICI 182,780. Cancer Res 53: 3229-3232.

327. Bronzert DA, Greene GL, Lippman ME (1985) Selection and characterization of a breast cancer cell line resistant to the antiestrogen LY 117018. Endocrinology 117: 1409-1417.

328. Tanner M, Kapanen AI, Junttila T, Raheem O, Grenman S, et al. (2004) Characterization of a novel cell line established from a patient with Herceptinresistant breast cancer. Mol Cancer Ther 3: 1585-1592.

329. Cummins TD, Barati MT, Coventry SC, Salyer SA, Klein JB, et al. (2010) Quantitative mass spectrometry of diabetic kidney tubules identifies GRAP as a novel regulator of TGF-beta signaling. Biochim Biophys Acta 1804: 653661. 
330. Nesvizhskii AI, Vitek O, Aebersold R (2007) Analysis and validation of proteomic data generated by tandem mass spectrometry. Nat Methods 4: 787797.

331. Huang da W, Sherman BT, Lempicki RA (2009) Systematic and integrative analysis of large gene lists using DAVID bioinformatics resources. Nat Protoc 4: 44-57.

332. Huang da W, Sherman BT, Lempicki RA (2009) Bioinformatics enrichment tools: paths toward the comprehensive functional analysis of large gene lists. Nucleic Acids Res 37: 1-13.

333. Kim LS, Kim JH (2011) Heat shock protein as molecular targets for breast cancer therapeutics. J Breast Cancer 14: 167-174.

334. Garrido C, Brunet M, Didelot C, Zermati Y, Schmitt E, et al. (2006) Heat shock proteins 27 and 70: anti-apoptotic proteins with tumorigenic properties. Cell Cycle 5: 2592-2601.

335. Ciocca DR, Oesterreich S, Chamness GC, McGuire WL, Fuqua SA (1993) Biological and clinical implications of heat shock protein 27,000 (Hsp27): a review. J Natl Cancer Inst 85: 1558-1570.

336. Calderwood SK, Khaleque MA, Sawyer DB, Ciocca DR (2006) Heat shock proteins in cancer: chaperones of tumorigenesis. Trends in biochemical sciences 31: 164-172.

337. Beere HM (2001) Stressed to death: regulation of apoptotic signaling pathways by the heat shock proteins. Sci STKE 2001: re1.

338. Al-Madhoun AS, Chen YX, Haidari L, Rayner K, Gerthoffer W, et al. (2007) The interaction and cellular localization of HSP27 and ERbeta are modulated by 17beta-estradiol and HSP27 phosphorylation. Mol Cell Endocrinol 270: 33-42.

339. Razandi M, Pedram A, Levin ER (2010) Heat shock protein 27 is required for sex steroid receptor trafficking to and functioning at the plasma membrane. Molecular and cellular biology 30: 3249-3261.

340. Porter W, Wang F, Wang W, Duan R, Safe S (1996) Role of estrogen receptor/Sp1 complexes in estrogen-induced heat shock protein 27 gene expression. Mol Endocrinol 10: 1371-1378.

341. Ciocca DR, Calderwood SK (2005) Heat shock proteins in cancer: diagnostic, prognostic, predictive, and treatment implications. Cell stress \& chaperones 10: 86-103.

342. Hansen RK, Parra I, Lemieux P, Oesterreich S, Hilsenbeck SG, et al. (1999) Hsp27 overexpression inhibits doxorubicin-induced apoptosis in human breast cancer cells. Breast cancer research and treatment 56: 187-196.

343. Zhang D, Wong LL, Koay ES (2007) Phosphorylation of Ser78 of Hsp27 correlated with HER-2/neu status and lymph node positivity in breast cancer. Mol Cancer 6: 52.

344. Li H, Villalobo A (2002) Evidence for the direct interaction between calmodulin and the human epidermal growth factor receptor. Biochem J 362: 499-505.

345. White CD, Li Z, Sacks DB (2011) Calmodulin binds HER2 and modulates HER2 signaling. Biochimica et biophysica acta 1813: 1074-1082. 
346. Ivanova M, Abner S, Pierce W, Jr., Klinge C (2011) Ligand-dependent differences in estrogen receptor beta-interacting proteins identified in lung adenocarcinoma cells corresponds to estrogenic responses. Proteome Sci 9: 60 .

347. Castoria G, Migliaccio A, Nola E, Auricchio F (1988) In vitro interaction of estradiol receptor with Ca2+-calmodulin. Mol Endocrinol 2: 167-174.

348. Carlier L, Byrne C, Miclet E, Bourgoin-Voillard S, Nicaise M, et al. (2012) Biophysical studies of the interaction between calmodulin and the $R(2)(8)(7)-$ $\mathrm{T}(3)(1)(1)$ region of human estrogen receptor alpha reveals an atypical binding process. Biochem Biophys Res Commun 419: 356-361.

349. Li Z, Joyal JL, Sacks DB (2001) Calmodulin enhances the stability of the estrogen receptor. J Biol Chem 276: 17354-17360.

350. Bouhoute A, Leclercq G (1992) Antagonistic effect of triphenylethylenic antiestrogens on the association of estrogen receptor to calmodulin. Biochem Biophys Res Commun 184: 1432-1440.

351. Endoh H, Maruyama K, Masuhiro Y, Kobayashi Y, Goto M, et al. (1999) Purification and identification of p68 RNA helicase acting as a transcriptional coactivator specific for the activation function 1 of human estrogen receptor alpha. Mol Cell Biol 19: 5363-5372.

352. Watanabe M, Yanagisawa J, Kitagawa H, Takeyama K, Ogawa S, et al. (2001) A subfamily of RNA-binding DEAD-box proteins acts as an estrogen receptor alpha coactivator through the N-terminal activation domain (AF-1) with an RNA coactivator, SRA. EMBO J 20: 1341-1352. 


\section{APPENDIX I - SUPPLEMENTARY MATERIAL}

\section{Methods S1}

Transient transfection with pCOUP-TFII-FLAG. MCF-7 cells, purchased from ATCC, and used at passages $<10$, were transiently transfected with $2 \mu \mathrm{g}$ pCOUPTFII-FLAG using the P-20 program of the Amaxa Nucleofection System. Cells were 'serum-starved' in phenol red-free IMEM supplemented with 5\% dextran coated charcoal stripped FBS (DCC-FBS) for $24 \mathrm{~h}$ before treatment with vehicle control ethanol (EtOH) for $2 \mathrm{~h}$ as indicated in the experimental design (Fig. 1).

\section{Protein identification by multidimensional protein identification technology} (MudPIT). Proteins eluted from the FLAG-affinity gel (Fig. 1) were digested by trypsin, desalted with C18 spin columns, dried by speedvac, and fractionated by strong cation exchange (SCX). The desalted samples were dissolved with loading buffer containing $20 \%$ acetonitrile (ACN), $100 \mathrm{mM}$ acetic acid (HAc), and $2 \mathrm{mM}$ $\mathrm{NH}_{4}$ Acetate and then loaded on a pre-cleaned SCX cartridge from Michrom Bioresources. After washing twice, peptides were eluted stepwise with $20 \mu \mathrm{l}$ of $\mathrm{HAc} / \mathrm{NH}_{4} \mathrm{Ac}$ buffers with increasing ionic strength and $\mathrm{pH}$ (15 buffers and up to 10 mM HAc/615 mM NH $4 \mathrm{Ac}, \mathrm{pH}$ 6.5). The SCX fractions were concentrated by speedvac and diluted to $7 \mu \mathrm{l}$ with $5 \% \mathrm{ACN} / 0.1 \%$ formic acid. $5 \mu \mathrm{l}$ of the samples

were loaded to CapLC capillary LC system from Waters and peptides in the samples 
were separated with a $3.5 \mu \mathrm{m}$ Symmetry C18 column $(75 \mu \mathrm{m} \times 150 \mathrm{~mm}$, Waters). MS/MS spectra of the peptides were acquired by Q-TOF mass spectrometer (Waters) in data dependent mode. Proteins were identified by comparing MS/MS spectra with sequences in Swiss-Prot database by ProteinLynx from Waters.

Immunofluorescence staining of pCOUP-TFII-FLAG. Cells were grown on coverslips, washed with PBS, fixed with cold methanol/acetone 1:1 for $5 \mathrm{~min}$, and washed twice with cold PBS. After blocking with $1 \%$ goat serum and $0.3 \%$ Triton X100 in PBS for 30 min, primary FLAG antibody (Sigma) was added (1: 300 dilution) for $1 \mathrm{~h}$. After removing the primary antibody, the cells were stained with secondary antimouse antibody labeled with Zenon ${ }^{\mathrm{TM}}$ Alexa Fluor 488 (Molecular Probes). Cells were then incubated with ProLong® Gold antifade reagent with DAPI (Molecular Probes). Images (Fig. S1) were captured using a Zeiss Axiovert200 inverted microscope with a 63x objective lens using AxioVision Release 4.3 software. 

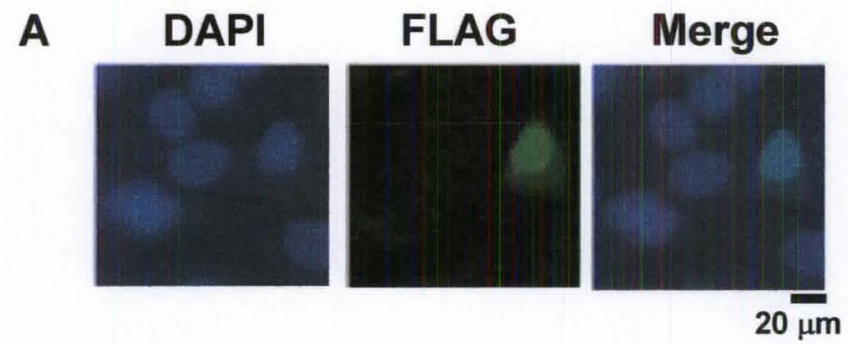

B Western blot

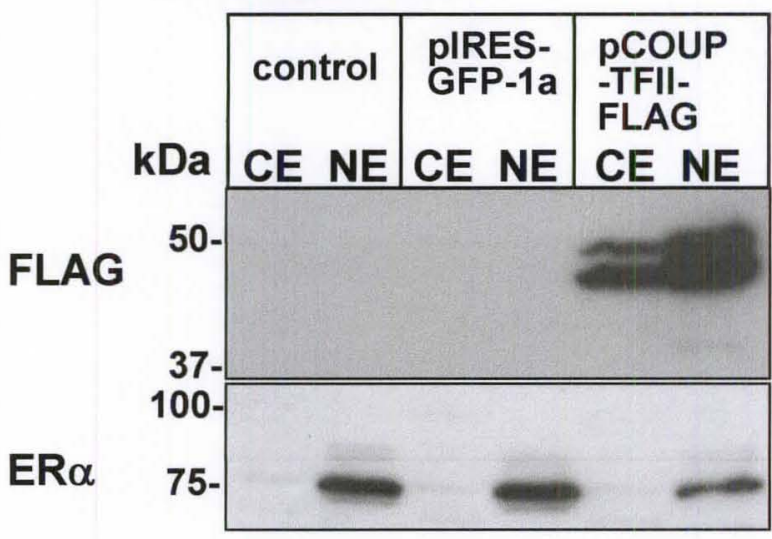

C Ponceau S staining

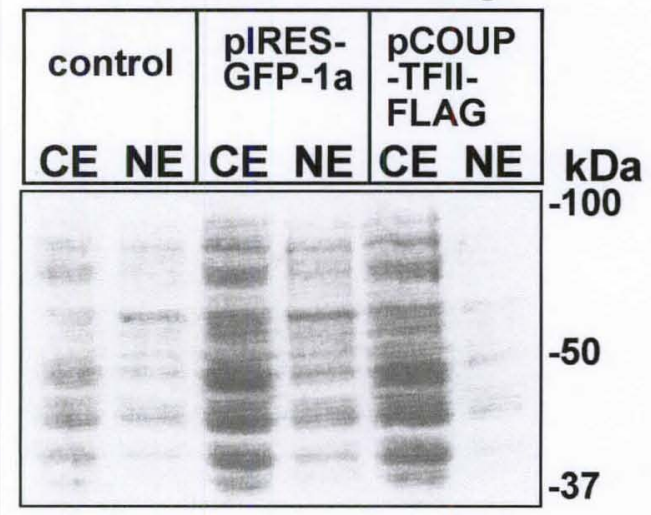

Figure S1: Nuclear localization of COUP-TFII in transfected MCF-7 cells.

A, MCF-7 cells were either non-transfected (control) or transfected with pCOUP-TFIIFLAG for $48 \mathrm{~h}$. Immunofluorescence staining was performed for FLAG as described in Supplementary Materials and Methods. Cells were counterstained with DAPI (blue) to image nuclei. The bar is $20 \mu \mathrm{m}$. Overlap images indicate localization of COUP-TFIIFLAG in the nucleus. B, Western blots of CE $(30 \mu \mathrm{g})$ or NE $(10 \mu \mathrm{g})$ from untransfected MCF-7 cells (control) or transfected with pIRES-GFP-1a parental vector or pCOUPTFII-FLAG with FLAG or ER $\alpha$ (AER320, ThermoFisher) antibodies. The two bands are likely due to protein degradation. C, Ponceau S staining shows protein levels. 


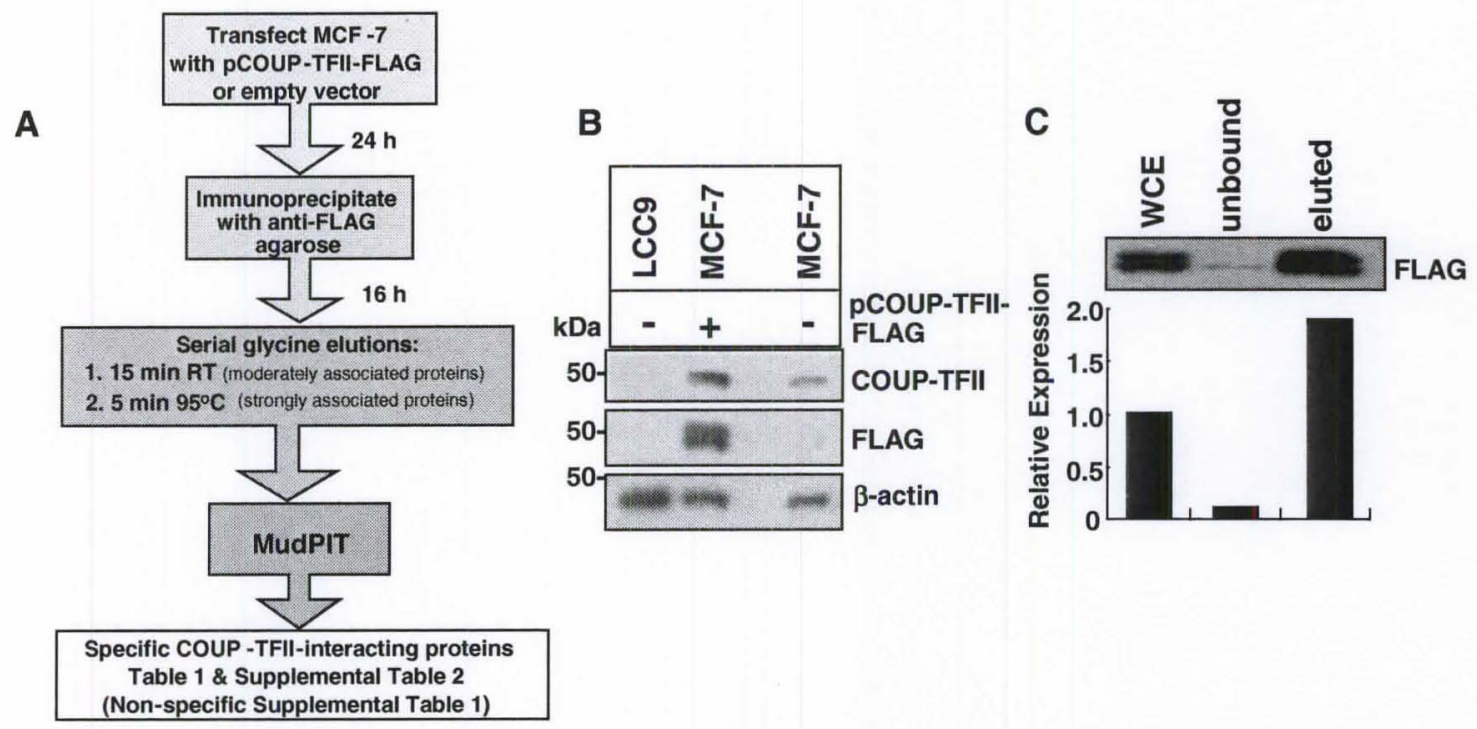

Figure S2: Overexpression of COUP-TFII-FLAG in MCF-7 cells and immunocapture of COUP-TF-FLAG by the anti-FLAG agarose affinity resin. A, Briefly, MCF-7 cells were transiently transfected with C-terminal FLAG-tagged COUP-TFII or empty vector for $24 \mathrm{~h}$ as described in Materials and Methods. WCEs were prepared and incubated with EZ view Red ANTI-FLAG M2 Affinity gel (Sigma) for 16 h. After rinsing, proteins were eluted with serial glycine elutions: $1.15 \mathrm{~min}$ at room temperature for proteins associating with immobilized COUP-TFII-FLAG with moderate affinity and $2.5 \mathrm{~min}$ at $95^{\circ} \mathrm{C}$ to elute proteins bound to the immobilized COUP-TFIIFLAG with high affinity. Immunoprecipitating proteins were analyzed by MudPIT. Nonspecific proteins were subtracted from the total interacting proteins to identify proteins specifically interacting with COUP-TFII-FLAG. B, $30 \mu \mathrm{g}$ of WCE from LCC9, MCF-7 and MCF-7 cells transiently transfected with pCOUP-TFII-FLAG were separated by SDS-PAGE and western blots were performed for COUP-TFII, FLAG and b-actin. Quantitation of the COUP-TFII/ $\beta$-actin in lanes 2 and 3 indicate a 2 -fold increase COUPTFII in the transfected cells. TAM-R LCC9 cells served as a negative control, as we reported lower COUP-TFII in LCC9 cells compared to parental MCF-7 cells (Riggs et al Cancer Res. 66: 10188-98, 2006). Note FLAG signal was only detected in the transfected cells (lane 2), indicating specificity. C, $1 \mathrm{mg}$ of protein in WCE from COUPTFII-FLAG over-expressing MCF-7 cells was immunocaptured on anti-FLAG agarose affinity beads. COUP-TFII and interacting proteins were eluted with $6 \mathrm{M}$ urea. $30 \mu \mathrm{g}$ of WCE were separated by SDS-PAGE in parallel to $30 \mu \mathrm{g}$ unbound IP supernatant and $30 \mu \mathrm{g}$ eluted protein. COUP-TFII-FLAG-affinity bead binding is confirmed by decreased FLAG in the Supernatant (flow-thru = unbound proteins) and enriched FLAG in the eluted samples. 


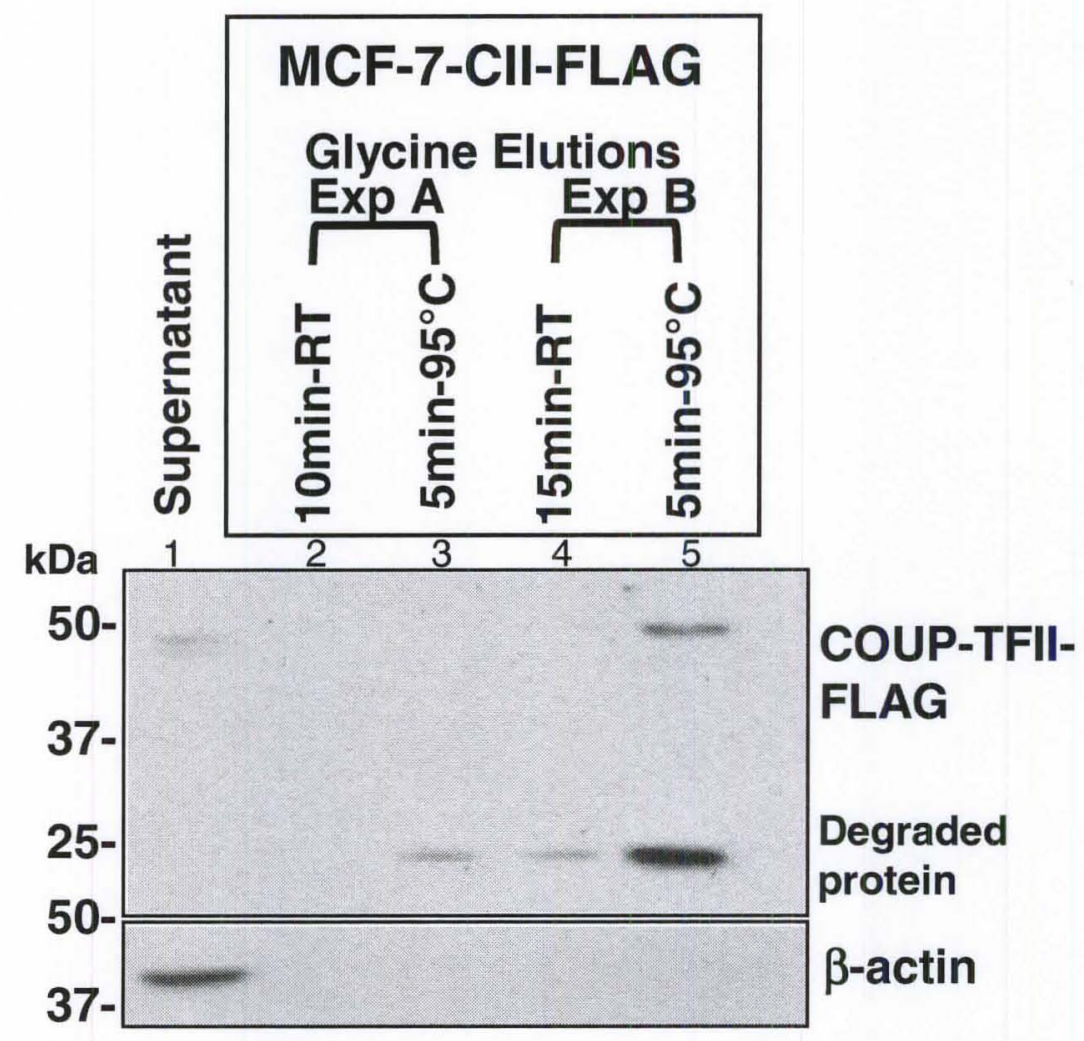

Figure S3: Testing elution methods for retrieval of COUP-TFII-FLAG.

$\sim 1 \mathrm{mg}$ of WCE from MCF-7 cells transfected with pCOUP-TFII-FLAG was immunocaptured on the anti-FLAG affinity resin and eluted with sequential glycine incubations: 1) 10 or $15 \mathrm{~min}$ room temperature (lanes 2 and 4 ) or $5 \mathrm{~min}$. at $95^{\circ} \mathrm{C}$ (lanes 3 and 5) in two different experiments (Exp A or B), as indicated (lanes 2-5). Samples of the indicated eluates were separated by SDS-PAGE and immunoblotted using anti-FLAG antibody. $20 \mu \mathrm{g}$ of the IP supernatant, containing unbound COUP-TFII-FLAG, was run in parallel as a control (lane 1). As seen in lane 5, incubation with glycine for $15 \mathrm{~min}$. followed by a $5 \mathrm{~min}$. incubation of fresh glycine at $95^{\circ} \mathrm{C}$ (Exp. B) eluted the most intact COUP-TFII-FLAG ( $\sim 50 \mathrm{kDa})$. The increased temperature and incubation time of glycine in Exp. B resulted in a FLAG-tagged degradation product(s) of $\sim 20 \mathrm{kDa}$ (lanes 3-5). 

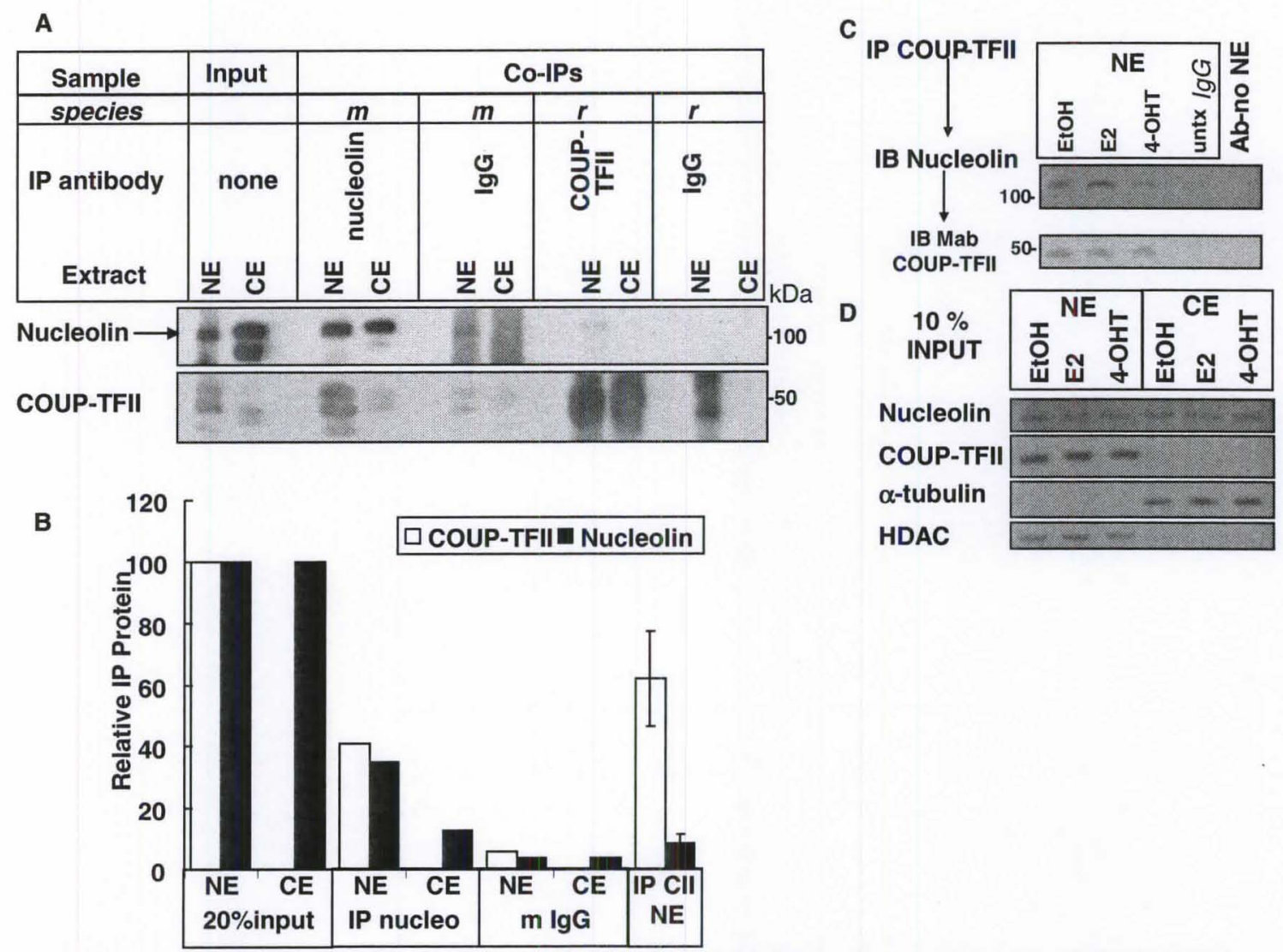

Figure S4: Endogenous nuclear nucleolin-COUP-TFII interaction in MCF-7 cells.

A, Equal amounts $(100 \mu \mathrm{g})$ of protein of $\mathrm{CE}$ and NE from MCF-7 cells were immunoprecipitated with nucleolin $\mathrm{mAB}$ (lanes 3 and 4), mouse (m) IgG (negative control for mAB, lanes 5 and 6), COUP-TFII antisera (lanes 7 and 8), or rabbit (r) IgG (negative control for IPs using COUP-TFII polyclonal antiserum, lanes 9 and 10), followed by western blot for nucleolin and COUP-TFII. 20\% $(20 \mu \mathrm{g})$ input NE and CE serve as loading controls (lanes 1 and 2). B, The relative amount of nucleolin and COUP-TFII in the nucleolin IP was plotted relative to expression of each protein in the input (set to 100). COUP-TFII in rabbit IgG IPs was not graphed because of the contamination of the heavy IgG chain (lanes 7 and 8, COUP-TFII blot). Western blots demonstrate that: 1) nucleolin interacts with COUP-TFII in the NE of MCF-7 cells (lane 7); 2) nucleolin is not IP'ed with rabbit IgG (lanes 9 and 10); 3) more COUP-TFII interacts with nucleolin in NE IP'ed with nucleolin antibody than with mouse IgG (lane 3 versus lane 5). C, MCF-7 cells were treated with EtOH, $10 \mathrm{nM} \mathrm{E}$, or $100 \mathrm{nM} 4-\mathrm{OHT}$ for $1 \mathrm{~h}$ prior to separation of NE and CE. $200 \mu \mathrm{g}$ of NE or CE were IP'ed with polyclonal COUP-TFII antibody and immunoblotted with a monoclonal antibody (mAB) against nucleolin. The blot was stripped and re-probed with $\mathrm{mAB}$ against COUP-TFII. D, 10\% input for NE and CE used in IP in part C. 


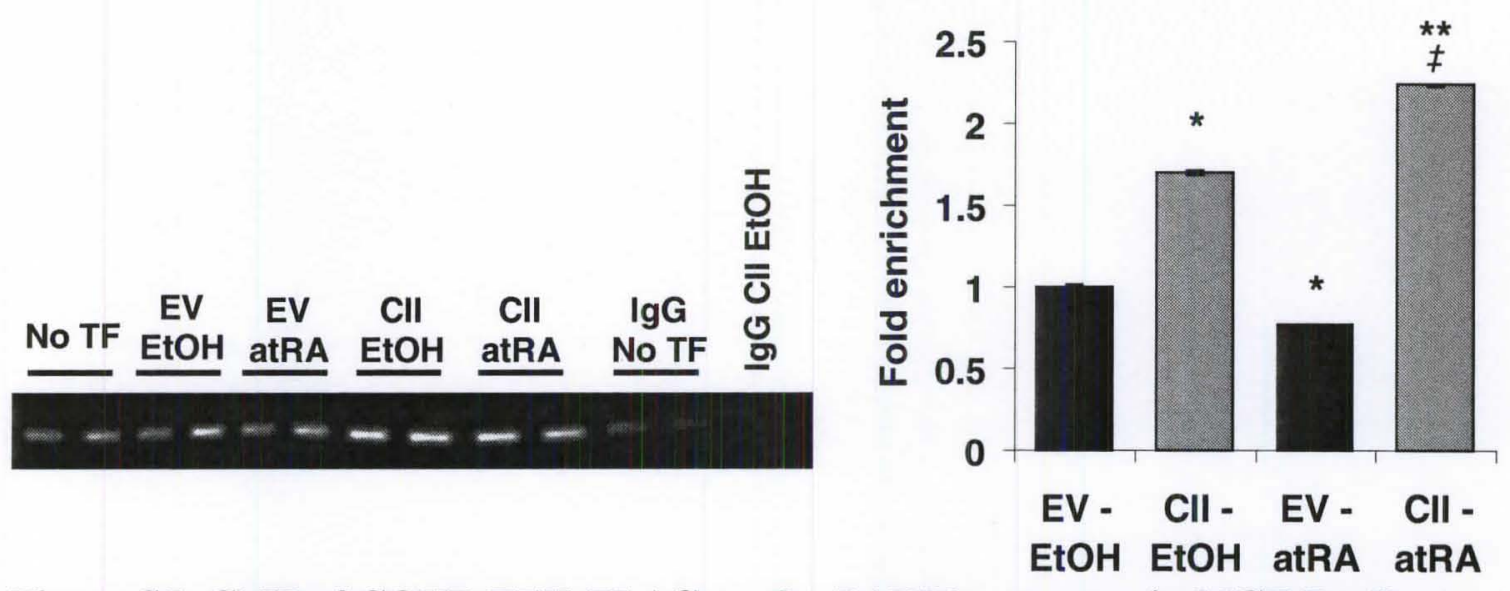

Figure S5: ChIP of COUP-TFII-FLAG on the RARB2 promoter in MCF-7 cells. A, Chromatin immunoprecipitation was performed in MCF-7 cells transfected with pIRESCOUP-TFII-FLAG or empty vector, serum starved for $48 \mathrm{~h}$, and treated with $1 \mu \mathrm{M}$ atRA for $1 \mathrm{~h}$. Following Q-PCR using primers to the RARB2 promoter as described in Materials and Methods, duplicate samples were run on a $2 \%$ agarose gel. B, Only EV EtOH set to 1. atRA increased COUP-TFII-FLAG binding to the RARB2 promoter $32 \%$. Signific. different $\mathrm{p}<0.05$ : * to EV - EtOH, ** to EV - atRA, $\neq$ to $\mathrm{CII}-\mathrm{EtOH}$. 
A
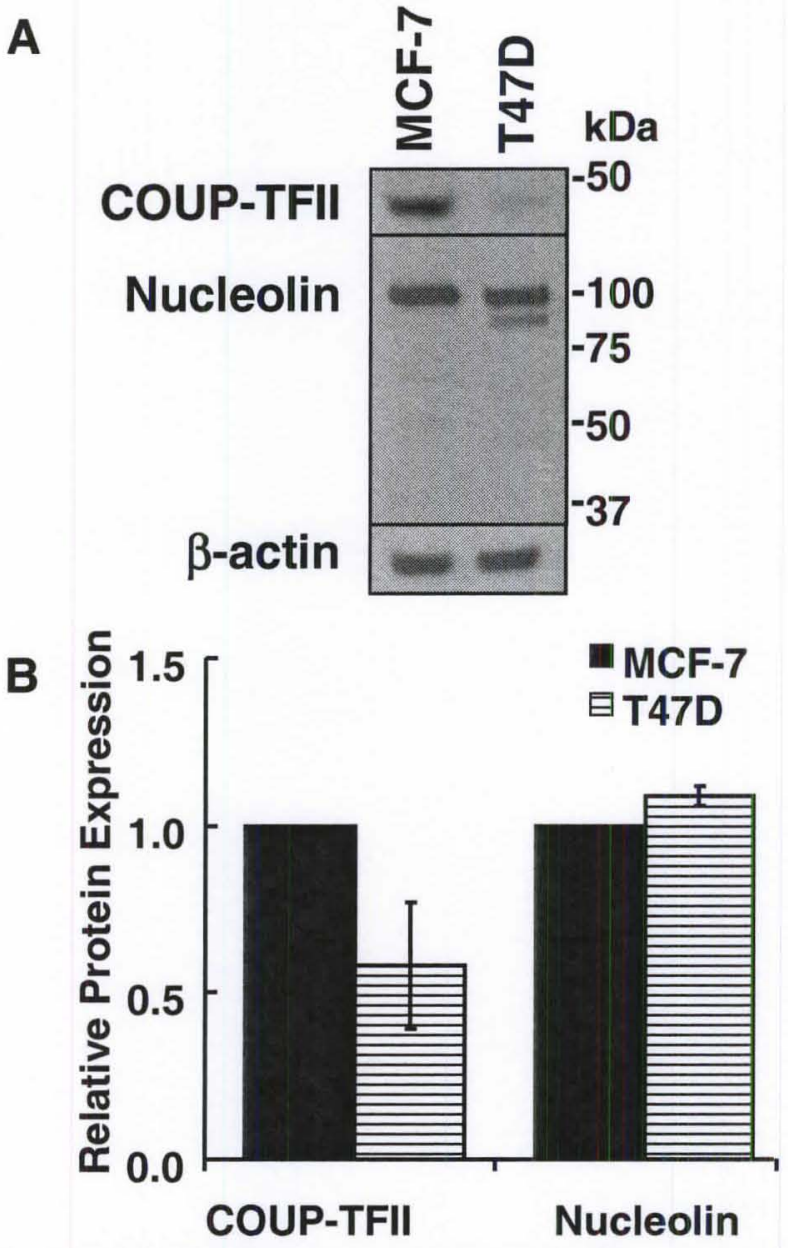

Figure S6: Expression of COUP-TFII and nucleolin in T47D and MCF-7 cells. A, WCE $(50 \mu \mathrm{g})$ were Western blotted for COUP-TFII and nucleolin expression. The blot was stripped and re-probed for $\beta$-actin. B, The ratio of nucleolin $/ \beta$-actin and COUPTFII/ $\beta$-actin for each cell lines was plotted. These data are the average of 3 separate experiments. The lower COUP-TFII expression in T47D agrees with the higher CT values for $N R 2 F 2$ in T47D. 


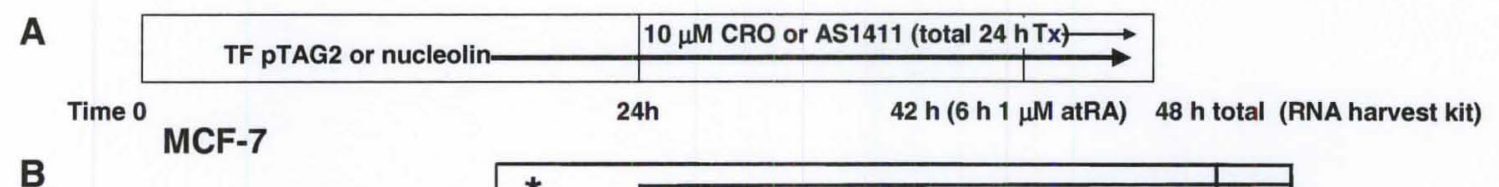

B

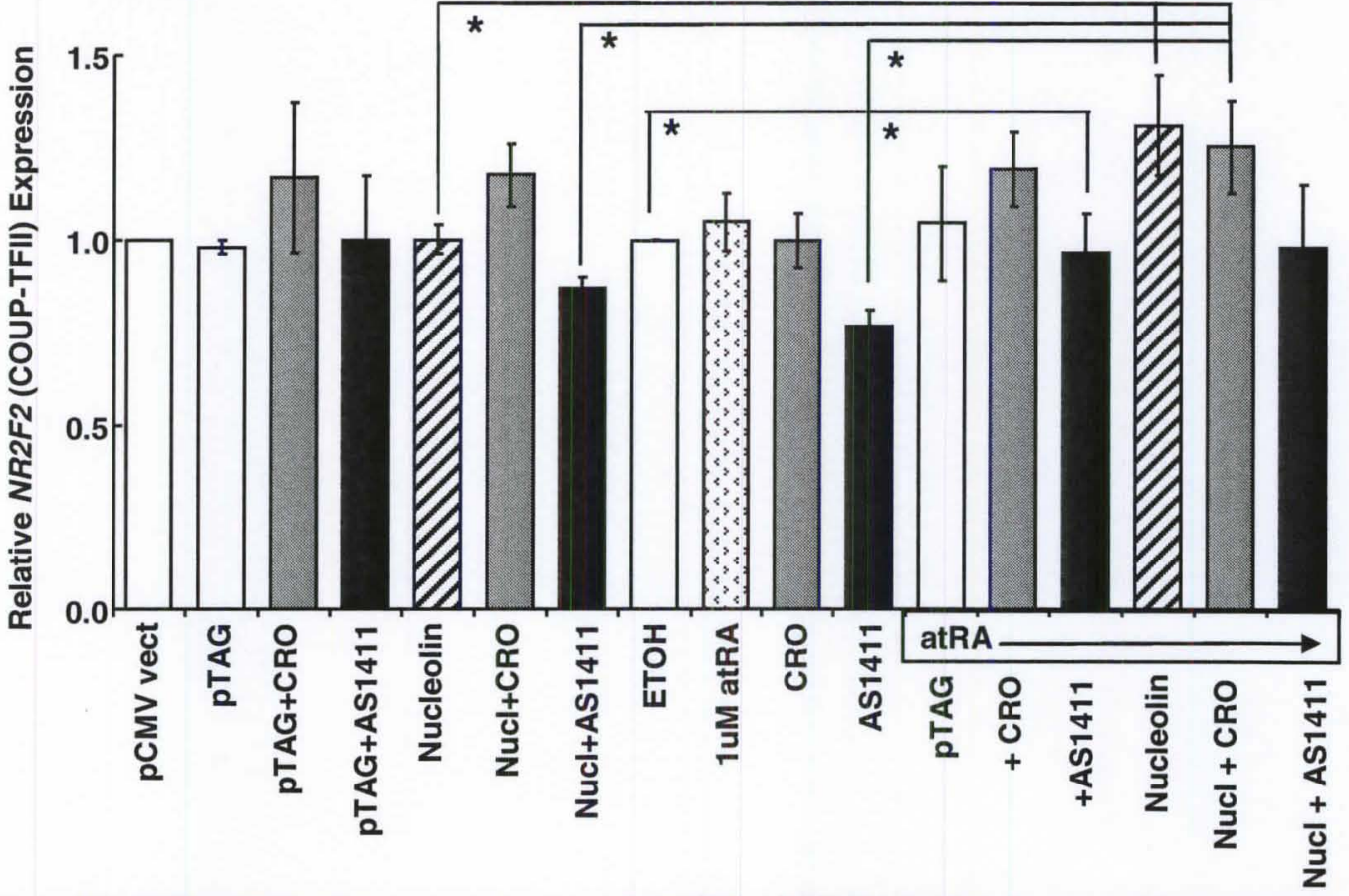

Figure S7: Effect of cell treatments on NR2F2 (COUP-TFII) expression in MCF-7 cells. A, Schematic diagram of transfection and treatment of MCF-7 cells. MCF-7 cells were transfected with equal amounts of pTAG2 control vector or pCMV-nucleolin for 24 h., treated with $10 \mu \mathrm{M} \mathrm{CRO}$ or AS1411, as indicted for $24 \mathrm{~h}$, and $1 \mu \mathrm{M}$ atRA was added for the last $6 \mathrm{~h}$. RNA was harvested and Q-PCR performed. B, NR2F2 values were normalized to GAPDH. Values are the average of 6 separate experiments \pm SEM. * signific. different, $\mathrm{p}<0.05$ in one way ANOVA followed by Bonferroni multiple comparison test. Note that there was no statistical difference between the pTAG+AS1411 versus Nucl+AS1411 or AS1411 sample measurements of $N R 2 F 2$. 


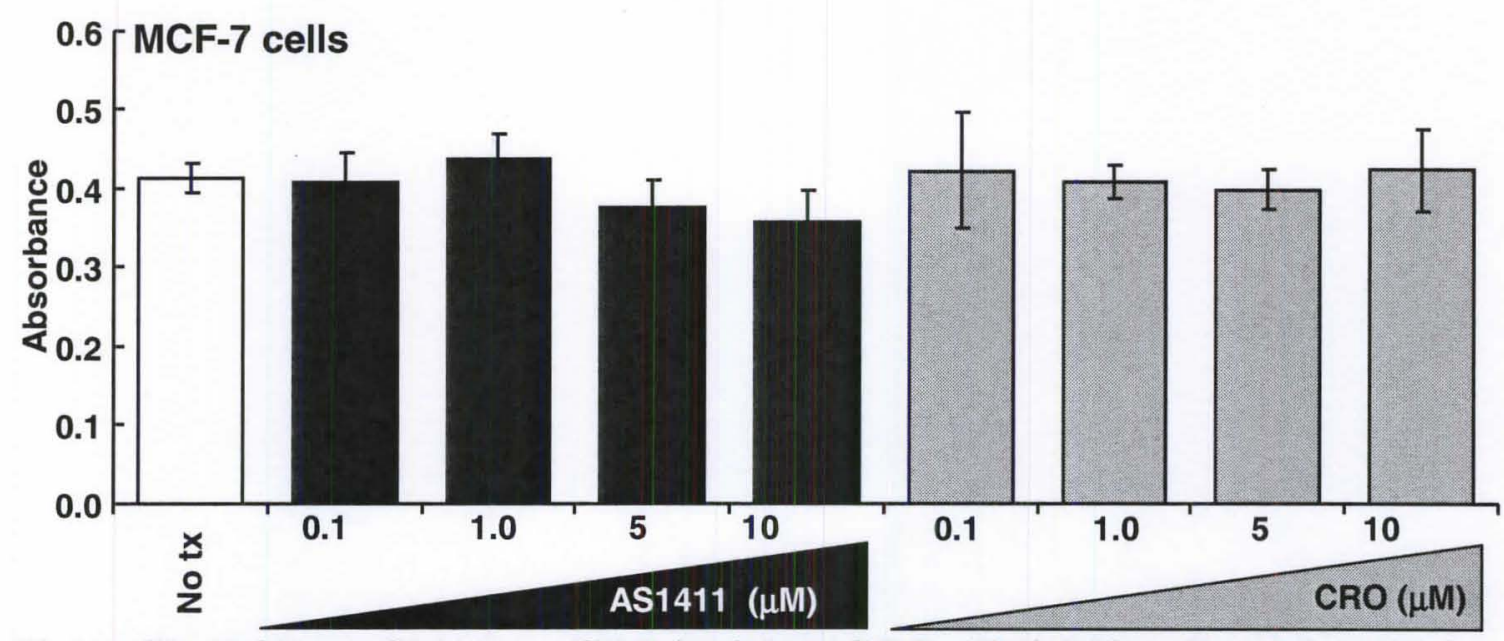

Figure S8: Neither AS1411 nor CRO inhibit MCF-7 cell viability after 4 d. MCF-7 cells were treated with the indicated concentrations of AS1411 or CRO and cell viability was measured by an MTT assay (A490nm, Promega CellTitre assay). Values are the average of 4 determinations \pm SEM. 

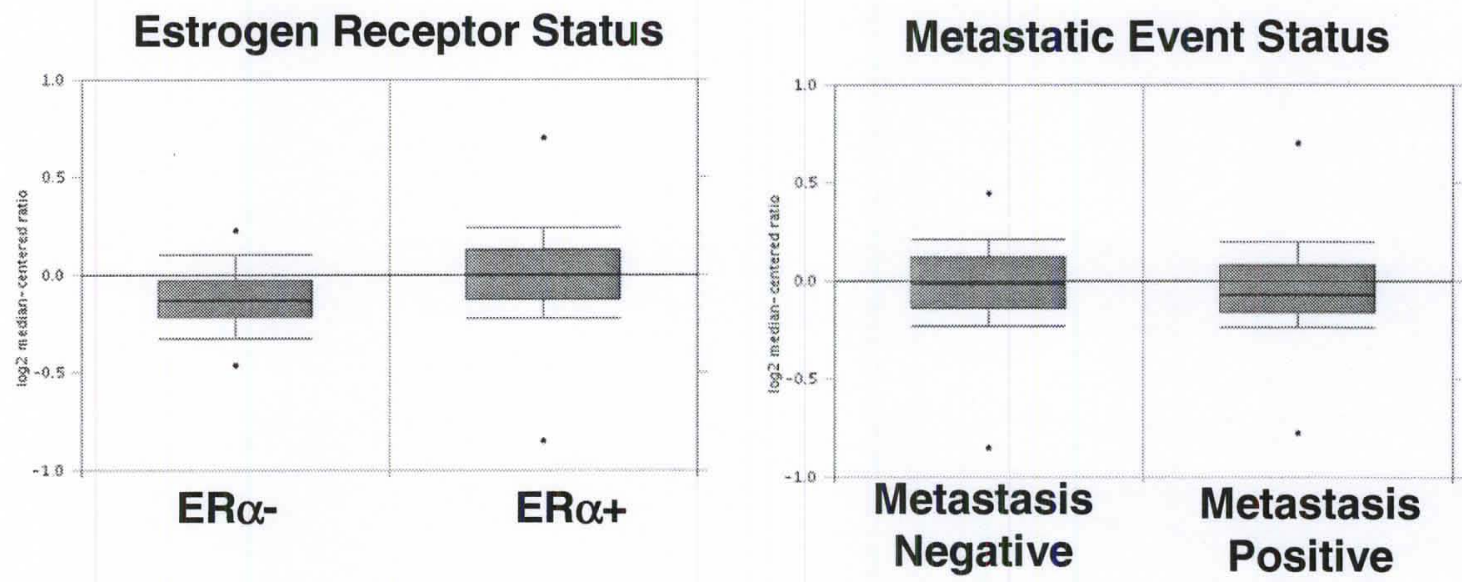

Figure S9: Oncomine examination of $N R 2 F 2$ expression in breast tumors. Data mining in Oncomine microarray data sets for NR2F2 in breast cancer identified that: A, $N R 2 F 2$ expression is significantly higher in ER $\alpha+$ breast tumors $(\mathrm{p}<0.007)$. B. NR2F2 is significantly lower in metastatic breast tumors $(\mathrm{p}<0.05)$. Data are from (van de Vijver MJ, He YD, van't Veer LJ, et al. A gene-expression signature as a predictor of survival in breast cancer. N Engl J Med 2002; 347: 1999-2009). 
Table S1: Identification of proteins in MCF-7 WCE that non-specifically (NS) interact with the anti-FLAG-affinity resin.

$1 \mathrm{mg}$ protein in WCE from EMPTY-FLAG vector-transfected MCF-7 cells was incubated with anti-FLAG affinity gel, eluted with $0.1 \mathrm{M}$ glycine, $\mathrm{pH} 3.5$ for $15 \mathrm{~min}$. at RT, and subjected to MudPIT peptide identification. Matched number (No) indicates the number of sequenced peptides that match the full length protein. Coverage indicates the $\%$ of the total protein matched.

\begin{tabular}{lccccc}
\hline Protein name & $\begin{array}{c}\text { Accession } \\
\text { (GI) }\end{array}$ & $\begin{array}{c}\text { Mass } \\
\text { (Mr) }\end{array}$ & pl & $\begin{array}{c}\text { Matched } \\
\text { (No) }\end{array}$ & $\begin{array}{c}\text { Coverage } \\
\text { (\%) }\end{array}$ \\
\hline Cytoskeletal proteins & & & & & \\
Actin cytoplasmic 1 & P60709 & 41710 & 5.4 & 4 & 15.5 \\
Tropomyosin alpha-4 chain & P67936 & 28505 & 4.7 & 4 & 17.3 \\
Tropomyosin alpha-3 chain & P06753 & 29015 & 4.8 & 4 & 18.1 \\
Myosin-9 & P35579 & 226392 & 4.6 & 4 & 3.0 \\
Tropomyosin beta chain & P07951 & 32970 & 4.7 & 5 & 23.6 \\
Tropomyosin alpha-1 chain & P09493 & 32689 & 4.7 & 4 & 14.8 \\
Myosin light polypeptide 6 & P60660 & 16788 & 4.6 & 3 & 22.7 \\
& & & & & \\
Histones & & & & & \\
Histone H4 & P62805 & 11229 & 11.4 & 3 & 29.4
\end{tabular}




\section{Table S2: Identification of proteins 'moderately' associated with COUP-TFII in}

MCF-7 cells.

$1 \mathrm{mg}$ protein in WCE from pCOUP-TFII-FLAG transfected, EtOH-treated MCF-7 cells was incubated with anti-FLAG affinity gel, eluted with $0.1 \mathrm{M}$ glycine, $\mathrm{pH} 3.5$ for $15 \mathrm{~h}$ at RT, and subjected to MudPIT peptide identification. Matched number (No) indicates the number of sequenced peptides that match the full length protein. Coverage indicates the $\%$ of the total protein matched. This table excludes proteins that were nonspecifically associated with the anti-FLAG affinity gel as summarized in Table S1.

\begin{tabular}{|c|c|c|c|c|c|}
\hline Protein name (gene) & $\begin{array}{l}\text { Accession } \\
\text { (GI) }\end{array}$ & $\begin{array}{l}\text { Mass } \\
(\mathrm{Mr})\end{array}$ & pl & $\begin{array}{l}\text { Matched } \\
\text { (No) }\end{array}$ & $\begin{array}{c}\text { Coverage } \\
(\%)\end{array}$ \\
\hline \multicolumn{6}{|l|}{ Ribonucleoproteins } \\
\hline $\begin{array}{l}\text { Heterogeneous nuclear } \\
\text { ribonucleoprotein D0 }\end{array}$ & Q14103 & 22435 & 11.3 & 2 & 10.2 \\
\hline \multicolumn{6}{|l|}{ Histones } \\
\hline Histone H2A.Z & P0COS5 & 13414 & 10.9 & 2 & $7.9-16$ \\
\hline Core histone macro-H2A.1 & 075367 & 39445 & 10.1 & 2 & 5.4 \\
\hline Histone $\mathrm{H} 1.5$ & P16401 & 22435 & 11.3 & 3 & 10.2 \\
\hline Histone H1.2 & P16403 & 21221 & 11.3 & 3 & 10.8 \\
\hline Histone H3.1 & P68431 & 15263 & 11.3 & 2 & 10.4 \\
\hline \multicolumn{6}{|l|}{ Other Proteins } \\
\hline $\begin{array}{l}\text { Heat Shock } 70 \text { kDa protein B } \\
\text { (HSPA7) }\end{array}$ & P48741 & 26890 & 7.6 & 3 & 16.2 \\
\hline $\begin{array}{l}\text { Heat Shock } 70 \text { kDa protein } 1 \\
\text { HSP70 (HSPA1A) }\end{array}$ & P08107 & 70009 & 5.6 & 3 & 6.2 \\
\hline $\begin{array}{l}\text { Chromobox Protein Homolog } 3 \\
(C B X 3)\end{array}$ & Q13185 & 19708 & 5.1 & 3 & 6.4 \\
\hline
\end{tabular}



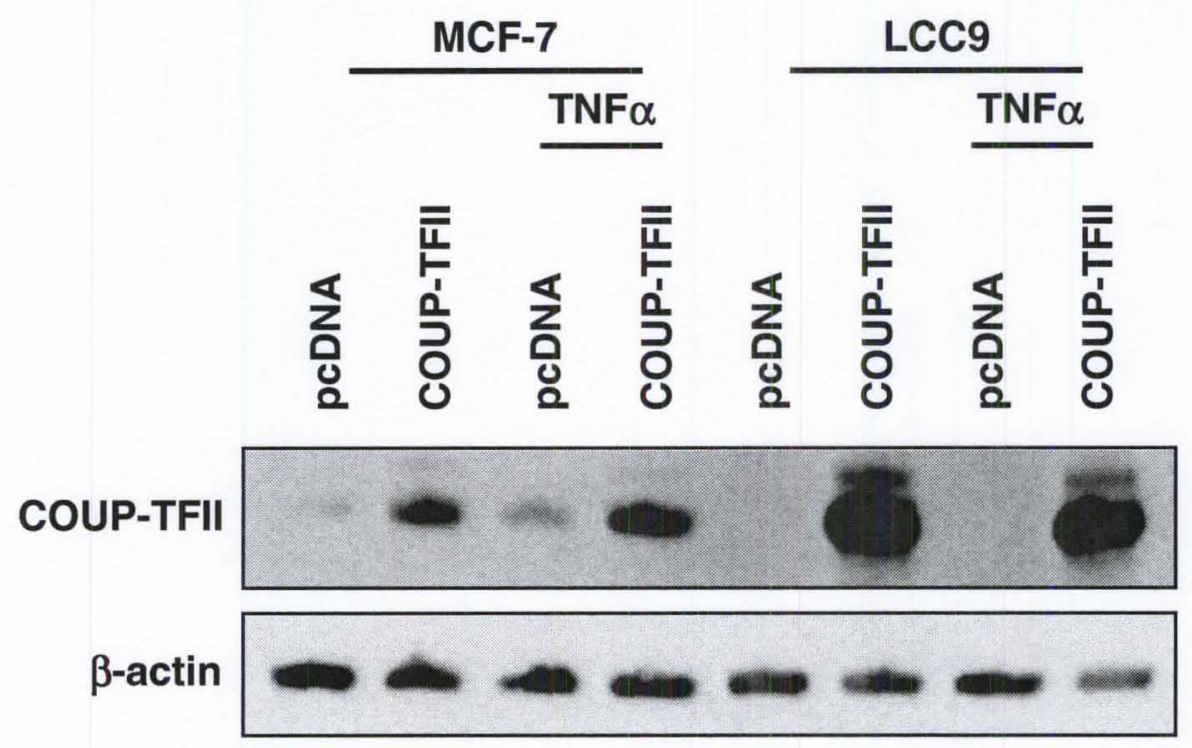

Figure S10. Expression of COUP-TFII in transfected MCF-7 and LCC9 cells. MCF-7 and LCC9 cells were transfected with pcDNA3.1 or pcDNA3.1-COUP-TFII for $48 \mathrm{~h}$ and treated with $10 \mathrm{ng} / \mathrm{ml} \mathrm{TNF} \alpha$ for $6 \mathrm{~h}$ before preparing whole cell extracts.

Expression of COUP-TFII was analyzed by western blot and compared to $\beta$-actin control. 


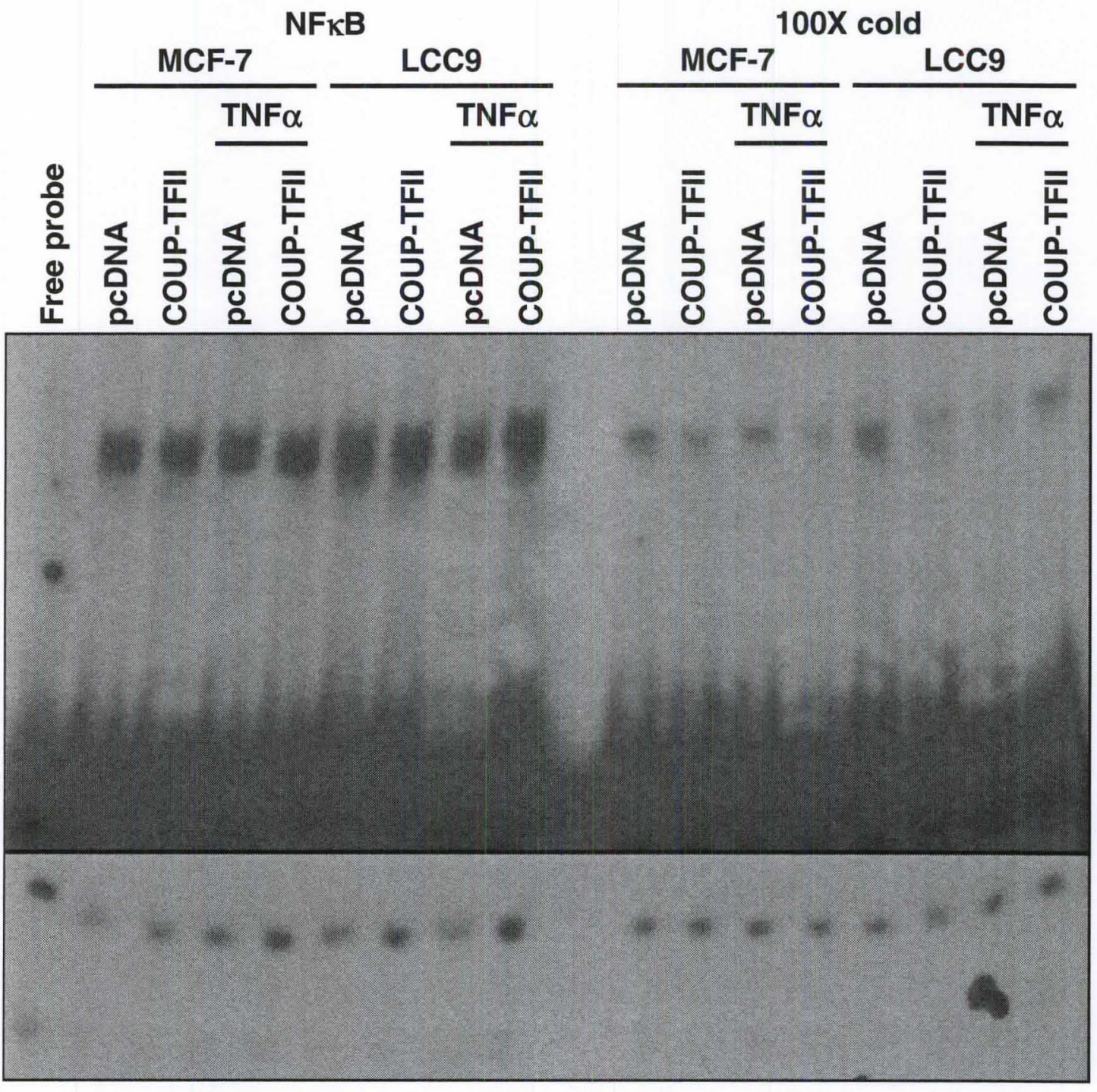

Figure S11. Specificity of protein complex binding to NFאB-RE DNA probe. Full EMSA picture from Fig. 6B. $25 \mu \mathrm{g}$ NE prepared from MCF-7 and LCC9 cells transfected with pcDNA3.1 or pcDNA3.1-COUP-TFII for $48 \mathrm{~h}$ and treated with TNF $\alpha$ for $6 \mathrm{~h}$ was incubated with $\left[{ }^{32} \mathrm{P}\right] \mathrm{NF} \kappa \mathrm{B}-\mathrm{RE}$ DNA probe $+/-100 \mathrm{X}$ cold unlabeled DNA probe for $1 \mathrm{~h}$ to perform EMSA. Binding to $\left[{ }^{32} \mathrm{P}\right] \mathrm{NF}$ KB-RE DNA probe is inhibited by the addition of excess cold DNA probe. 


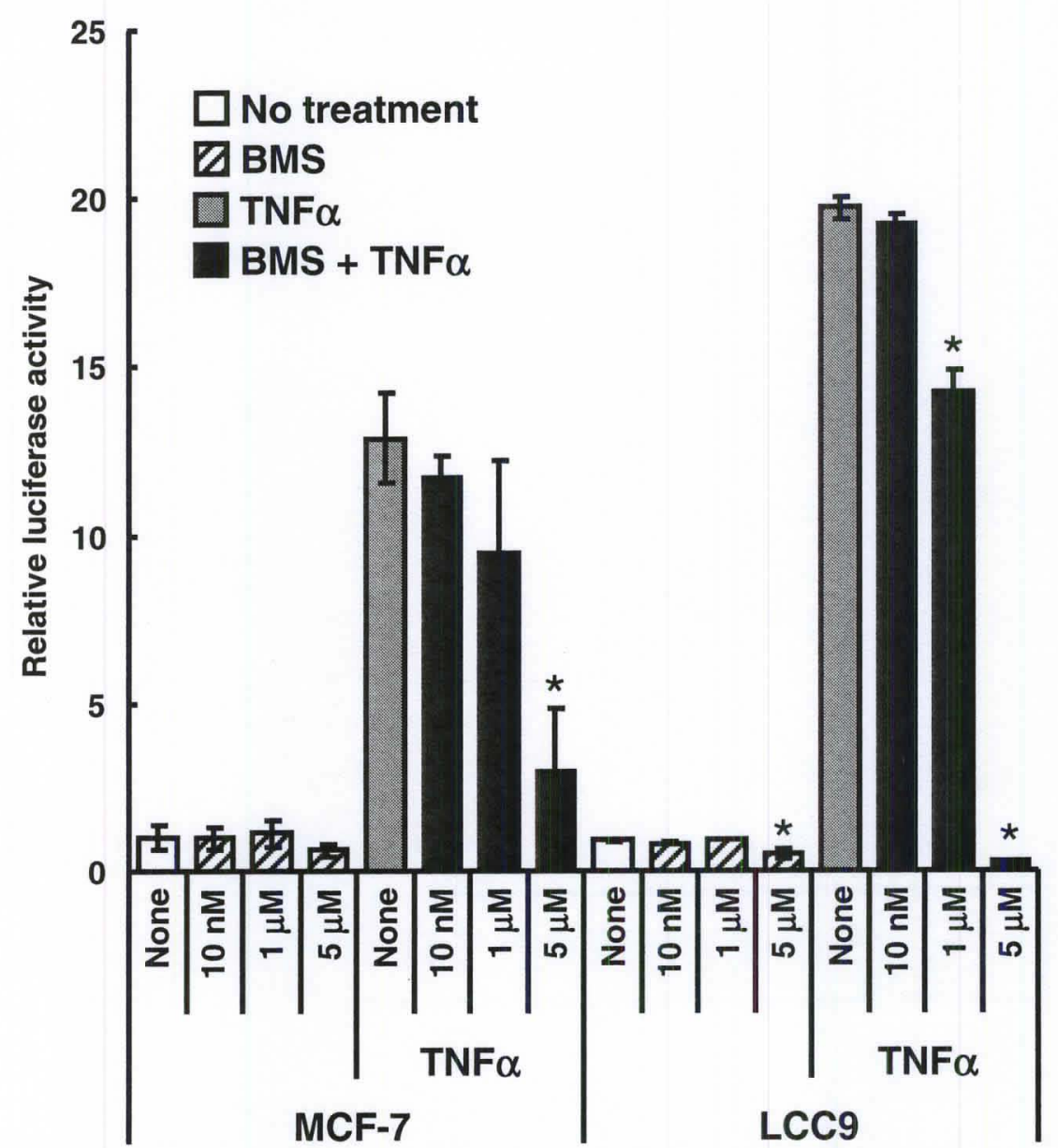

Figure S12. BMS-345541 inhibits NFkB activity in MCF-7 and LCC9 cells.

MCF-7 and LCC9 cells were serum-starved for $24 \mathrm{~h}$ before being transfected with a $\mathrm{NF \kappa B}$ luciferase reporter for $48 \mathrm{~h}$ and treated with various concentrations of an NFKB inhibitor, BMS-345541 +/- $10 \mathrm{ng} / \mathrm{ml}$ TNF $\alpha$ for $6 \mathrm{~h}$. Dual luciferase assay was performed to verify that the BMS-345541 inhibited NFKB activity at these concentrations. Firefly luciferase values are shown relative to pGL4-hRluc-TK Renilla (Promega). Note higher NFKB activity in serum-starved MCF-7 cells as compared to normal media (Figure 1). *: $\mathrm{P}<0.05$ versus DMSO control for each cell line. 
CURRICULUM VITAE

Lacey M. Litchfield

Department of Biochemistry and Molecular Biology

University of Louisville School of Medicine

Louisville, KY 40292

\section{EDUCATION}

2004-08

B.S. Biology, concentration Genetics/Subcellular (summa cum laude), University of Louisville, Louisville, KY GPA 3.875

2008-11 M.S. Biochemistry and Molecular Biology, University of Louisville School of Medicine, Louisville, KY

2008-13 Ph.D. Biochemistry and Molecular Biology, University of Louisville School of Medicine, Louisville, KY GPA 3.948

\section{RESEARCH EXPERIENCE}

2007-08

Undergraduate Research

The role of nucleolin in the mechanism of novel anticancer agent, AS1411

University of Louisville School of Medicine

Mentor: Dr. Paula J. Bates

2008-13

Doctoral Research

COUP-TFII and its interacting proteins in breast cancer and

endocrine resistance

Department of Biochemistry and Molecular Biology

University of Louisville School of Medicine

Mentor: Dr. Carolyn M. Klinge

\section{HONORS}

Golden Key International Honour Society 
2008

2008

2008-10

$2010-12$

2012

2013

2013
University honors scholar, University of Louisville, Louisville, KY Summa cum laude graduate, University of Louisville, Louisville, $\mathrm{KY}$ Integrated Programs in Biomedical Sciences Fellowship NIEHS T32-ES011564 Predoctoral Fellowship First place award in poster competition at Research!Louisville, University of Louisville School of Medicine, Louisville, KY, September 18, 2012. Litchfield, L.M. and Klinge, C.M. COUPTFII suppresses NFkB activation in endocrine-resistant breast cancer cells. (Abstract \#56)

John M. Houchens Award for most meritorious dissertation Graduate Dean's Citation

\section{PUBLICATIONS}

1. Litchfield, L.M., Riggs, K.A., Hockenberry, A.M., Oliver, L.D., Barnhart, K.G., Cai, J., Pierce, W.M., Ivanova, M.M., Bates, P.J., Appana, S.N., Datta, S., Kulesza, P., McBryan, J., Young, L.S., Klinge, C.M. Identification and Characterization of Nucleolin as a COUP-TFII Coactivator of Retinoic Acid Receptor $\beta$ Transcription in Breast Cancer Cells. PLOS ONE 7(5): e38278, 2012. doi:10.1371/journal.pone.0038278

2. Litchfield, L.M. and Klinge, C.M. Multiple roles of COUP-TFII in cancer initiation and progression. Journal of Molecular Endocrinology, 49(3): R135-48, 2012. doi:10.1530/JME-12-0144

3. Manavalan, T.T.*, Teng, Y.*, Litchfield, L.M.*, Muluhngwi, P., Al-Rayyan, N., Klinge, C.M. Reduced expression of miR-200 family members contributes to antiestrogen resistance in LY2 human breast cancer cells. PLOS ONE, In publication. *equal contribution

4. Litchfield, L.M. and Klinge, C.M. COUP-TFII inhibits NFkB activation in breast cancer cells and restores endocrine sensitivity. In submission.

\section{PROFESSIONAL MEMBERSHIPS}

2011-Present

2012-Present

2012-Present 2012-Present
The Endocrine Society

American Association for Cancer Research

Women in Cancer Research

Kentucky Academy of Science 


\section{ORAL PRESENTATIONS}

1. Litchfield, L.M., Emberts, C.G., and Klinge, C.M. COUP-TFII-Nucleolin Interaction Regulates RAR $\beta 2$ Expression in Human Breast Cancer Cells. Oral Presentation at the 2011 Colloquium of Biochemistry and Molecular Biology, University of Louisville School of Medicine, August 26, 2011.

\section{POSTER PRESENTATIONS}

\section{Abstracts of poster presentations (national):}

1. Litchfield, L.M., Emberts, C.G., and Klinge, C.M. COUP-TFII-Nucleolin Interaction Regulates RAR[beta]2 Expression in Human Breast Cancer Cells (Abstract P2-10) Abstracts of the 93rd annual meeting of the Endocrine Society, Boston, MA, June 5, 2011.

2. Litchfield, L.M., Cai, J., Pierce, W.M., Appana, S.N., Bates, P.J., Datta, S., Kulesza, P., Young, L.S., and Klinge, C.M. Identification and functional characterization of COUP-TFII-nucleolin interaction in breast cancer cells and tumors. AACR Advances in Breast Cancer Research: Genetics, Biology, and Clinical Applications meeting, San Francisco, CA, October 14, 2011.

3. Litchfield, L.M. and Klinge, C.M. COUP-TFII suppresses NFאB activation in endocrine-resistant breast cancer cells. (Abstract \#2214) The 103rd annual meeting of the American Association for Cancer Research, Chicago, IL, April 2, 2012.

4. Litchfield, L.M. and Klinge, C.M. COUP-TFII suppresses NFאB activation in endocrine-resistant breast cancer cells. (P6-04-30) CTRC-AACR San Antionio Breast Cancer Symposium, San Antonio, TX, December 8, 2012.

Abstracts of poster presentations (local):

1. Fox, J.M., Litchfield, L.M., Emberts, C.G., Clark, B.J., and Klinge, C.M. Characterization of the impact of nucleolin on COUP-TFII-mediated regulation of ER $\alpha, E R \beta, R A R \beta$ and StAR transcription in MCF-7 Human Breast Cancer Cells. (Abstract MED-19) Research!Louisville, University of Louisville School of Medicine, Louisville, KY, October 14, 2009.

2. Oliver, L.D., Litchfield, L.M., and Klinge, C.M. The effect of COUP-TFIInucleolin interaction on RAR 32 expression in human breast cancer cells. SROP poster presentation. University of Louisville School of Medicine, Louisville, KY, August 1, 2010.

3. Litchfield, L.M., Emberts, C.G., and Klinge, C.M. The effect of COUP-TFIInucleolin interaction on RAR $\beta 2$ expression in human breast cancer cells. (Abstract GRD-40) Research!Louisville, University of Louisville School of Medicine, Louisville, KY, October 13, 2010.

4. Oliver, L.D., Litchfield, L.M., and Klinge, C.M. The effect of COUP-TFIInucleolin interaction on RAR $\beta 2$ expression in human breast cancer cells. Posters at the Capitol. Frankfort, KY, February 10, 2011.

5. Litchfield, L.M., Emberts, C.G., and Klinge, C.M. The effect of COUP-TFII- 
nucleolin interaction on RAR $\beta 2$ expression in human breast cancer cells. $13^{\text {th }}$ annual Institute for Molecular Diversity and Drug Design (IMD3) Symposium, March 8, 2011.

6. Barnhart, K.G., Litchfield, L.M., and Klinge, C.M. Effect of COUP-TFII on RAR $\beta 2$ and NFKB Expression in Breast Cancer Cells. SROP poster presentation. University of Louisville School of Medicine, Louisville, KY, August 1, 2011.

7. Litchfield, L.M., Emberts, C.G., and Klinge, C.M. COUP-TFII-Nucleolin Interaction Regulates RAR 32 Expression in Human Breast Cancer Cells. (Abstract GRD-52) Research!Louisville, University of Louisville School of Medicine, Louisville, KY, October 11, 2011.

8. Litchfield, L.M., Emberts, C.G., and Klinge, C.M. COUP-TFII-Nucleolin Interaction Regulates RAR 32 Expression in Human Breast Cancer Cells. (Abstract \#76) $10^{\text {th }}$ annual Brown Cancer Center Retreat, Louisville, KY, October 28, 2011.

9. Litchfield, L.M. and Klinge, C.M. COUP-TFII suppresses NFkB activation in endocrine-resistant breast cancer cells. (Abstract \#31) $14^{\text {th }}$ annual Institute for Molecular Diversity and Drug Design (IMD3) Symposium, March 13, 2012.

10. Litchfield, L.M. and Klinge, C.M. COUP-TFII suppresses NFkB activation in endocrine-resistant breast cancer cells. (Abstract \#56) Research!Louisville, University of Louisville School of Medicine, Louisville, KY, September 18, 2012.

11. Litchfield, L.M. and Klinge, C.M. COUP-TFII suppresses NFkB activation in endocrine-resistant breast cancer cells. $11^{\text {th }}$ annual Brown Cancer Center Retreat, Louisville, KY, October 26, 2012.

12. Litchfield, L.M. and Klinge, C.M. COUP-TFII suppresses NFkB activation in endocrine-resistant breast cancer cells. $15^{\text {th }}$ annual Institute for Molecular Diversity and Drug Design (IMD3) Symposium, March 12, 2012. 